\title{
The results of a unique Nordic HAKK interlaboratory REAT comparison
}

\author{
Poulsen, Torben
}

Published in:

Acoustical Society of America. Journal

Link to article, DOI:

$10.1121 / 1.425267$

Publication date:

1999

Document Version

Publisher's PDF, also known as Version of record

Link back to DTU Orbit

Citation (APA):

Poulsen, T. (1999). The results of a unique Nordic HAKK interlaboratory REAT comparison. Acoustical Society of America. Journal, 105(2), 1129-1129. https://doi.org/10.1121/1.425267

\section{General rights}

Copyright and moral rights for the publications made accessible in the public portal are retained by the authors and/or other copyright owners and it is a condition of accessing publications that users recognise and abide by the legal requirements associated with these rights.

- Users may download and print one copy of any publication from the public portal for the purpose of private study or research.

- You may not further distribute the material or use it for any profit-making activity or commercial gain

- You may freely distribute the URL identifying the publication in the public portal

If you believe that this document breaches copyright please contact us providing details, and we will remove access to the work immediately and investigate your claim. 
Posters from various technical sessions remain on display in the Poster Gallery.

Posters from sessions which contain both lecture and poster presentations will be attended by the authors as listed below.

$$
\text { 10:00-12:00 }
$$

\begin{tabular}{|c|c|c|}
\hline 1pAA13 & Semidor, Catherine & Acoustical modifications of the interior design of a $50^{\prime}$ chapel \\
\hline 1pAA14 & Ivancevic, Bojan & The acoustical properties of large studios \\
\hline 1pAA15 & Tronchin, Lamberto & The sound quality of the Theatre Comunale in Treviso, Italy \\
\hline 2aAAa13 & Smigelschi, Marius & CAAD-Computer aided architectural and acoustical design \\
\hline 2aNSc9 & Picard, Michel & The confounding influence of collapsing the ear canal on audiological assessment of noise-exposed workers \\
\hline 2pAAa7 & Kramer, Peter S. & $\begin{array}{l}\text { Room acoustical measurements and architectural documentation of auditoriums in Berlin considering the } \\
\text { speech intelligibility in dependence of spatial distribution of sound fields }\end{array}$ \\
\hline 2pAAa8 & Galindo, Miguel & Simulated acoustics field in mudejar-gothic churches \\
\hline 2pAAb7 & Morimoto, Masayuki & $\begin{array}{l}\text { Appropriate band width and integration time for measurements of the degree of interaural cross-correlation } \\
\text { as a measure of apparent source width in concert halls }\end{array}$ \\
\hline 2pAAb8 & Stauskis, Vytautas J. & $\begin{array}{l}\text { The interrelation between the music sound clarity index C } 80 \text { and the subjective evaluation of the hall } \\
\text { acoustics }\end{array}$ \\
\hline 2pPAa10 & Ruhlich, I. & How much pressure drop is necessary for heat transfer \\
\hline 2pPAa11 & Le Polles, T. & Threshold condition for travelling wave excitation in an annular prime-mover \\
\hline 2pUWa11 & Hedlin, Michael A. H. & Surveying infrasonic noise on Oceanic Islands \\
\hline 2pUWa12 & Kulichkov, Sergey N. & On temporal and spatial variations of infrasonic signals at long distances from surface explosions \\
\hline $2 \mathrm{pUWb} 9$ & Hickman, Granger W. & Matched-field depth discrimination with wave sonar \\
\hline $3 \mathrm{aNSa} 5$ & Schulz, Detlef & Non-occupational noise and hearing capability of children and young adults \\
\hline $3 \mathrm{aNSa} 6$ & Domitrovic, Hrvoje & Noise levels in dental practice \\
\hline $3 \mathrm{aNSa} 7$ & Barham, Richard G. & The role of measurement standards in acoustics \\
\hline 3aUWa11 & Khelladi, Hassina & Analysis of boundaries' effects in the evaluation of transient ultrasonic fields scattered by a rigid reflector \\
\hline 3aUWa12 & Semenov, Andrew G. & Sound scattering by moving inhomogeneity \\
\hline $4 \mathrm{aAAb} 13$ & Masyukov, Valery A. & Noise transmission through a finite plate: physical approach \\
\hline 4aAAb14 & Rebureo, Manuel & Prediction equations of isolating systems: gypsum panels \\
\hline 4pAA13 & Jang, Jaehee & System development to reduce floor impact sound in apartment houses \\
\hline 4pAA14 & Kustner, Eberhard & $\begin{array}{l}\text { Additional improvement of air borne and impact sound insulation of ceilings in prefabricated standard } \\
\text { house-buildings }\end{array}$ \\
\hline 4pAA15 & Rodriguez-Manzo, Fausto & Towards a classification of acoustic comfort. A case study of a university classroom \\
\hline 5aAA13 & Legrand, Olivier & High frequency asymptotics and random matrix theory in reverberant rooms \\
\hline 5aAA14 & Morfey, Christopher L. & Mode counts for rooms and waveguides \\
\hline 5aAA15 & Picard Lopez, M. A. & $\begin{array}{l}\text { An extension of the transfer function method described in ASTM E 1050-90 for its use in semianechoic } \\
\text { conditions }\end{array}$ \\
\hline 5aAA16 & Dutilleux, Guillaume, J. A. & $\begin{array}{l}\text { Towards a global in situ method for materials acoustic impedance assessment at very low frequencies using } \\
\text { evolution strategy }\end{array}$ \\
\hline 5aAA17 & Bosmans, Ivan & Sound absorption properties of suspended ceilings with plastic foils \\
\hline $5 \mathrm{pAA} 8$ & Becker, Jorg & Four-microphone-array measurements combined with geometrical room acoustic simulation technique \\
\hline 5pAA9 & Gomes, Marcio H. & $\begin{array}{l}\text { On the accuracy of the assessment of room acoustical parameters using MLS technique and ray-tracing } \\
\text { simulation }\end{array}$ \\
\hline
\end{tabular}

Also, the following poster sessions are scheduled:

Poster Session $3 \mathrm{aAOa}$

Poster Session $3 \mathrm{aPPb}$

Poster Session 3aUWc 


\title{
Session 3aABa
}

\section{Animal Bioacoustics: Evolution in Hearing and Sound Production I}

\author{
Robert J. Dooling, Cochair \\ Department of Psychology, University of Maryland, College Park, Maryland 20742, USA \\ Annemarie Surlykke, Cochair \\ Center for Sound Communication, Institute of Biology, Odense University, DK-5230 Odense M., Denmark
}

Invited Papers

\begin{abstract}
8:00
3aABa1. The evolution of tympanate hearing from proprioceptors in ancient grasshoppers. Heiner Roemer (Zoology, Karl-Franzens Univ., A-8010 Graz, Austria) and Moira J. van Staaden (Bowling Green State Univ., Bowling Green, OH 43403)

The ears of modern insects occur on rather different body parts, from head, to thorax, to abdomen, and there is evidence from comparative anatomy and embryology in divergent taxa that they have evolved from proprioreceptors responding to stretch or vibration. Receptor cells of these ears are directly or indirectly coupled to thin cuticular membranes (tympana), which are considered to play important roles in sensitivity and frequency tuning of such ears. This report is on a new set of hearing organs in an ancient grasshopper (Bullacris membracioides) without the typical tympanum, but a sensitivity exceeding most tympanate ears. As a result, males are capable of signaling acoustically over up to two $\mathrm{km}$. In addition, within single individuals of this species, six pairs of serially repeated abdominal ears exist which all respond to biologically relevant frequencies and intensities. Altogether, anatomical comparison and results of neurophysiological and behavioral experiments provide strong evidence for the transition in function from proprioreception to airborne sound reception, and for the selective advantage during the evolutionary development of a complex ear structure. [Work supported by Austrian Science Foundation.]
\end{abstract}

8:30

3aABa2. Evolution of vocal communication in the gray treefrog complex. H. Carl Gerhardt (Univ. of Missouri, Columbia, Columbia, MO 65211, bioscarl@ showme.missouri.edu)

The evolution of communication requires changes in both signals and the criteria used by receivers to select signals. The question arises: Do changes occur first in signals, first in receivers, or are tight co-evolutionary changes required in both signals and receivers? In the gray treefrog complex, spectral properties of calls are conserved: in both the diploid ancestral species (Hyla chrysoscelis) and in derived, tetraploid lineages (nominally, $\mathrm{H}$. versicolor) there are two spectral peaks: a dominant band centered at about $2.4 \mathrm{kHz}$, and a secondary band $(-8$ to $-10 \mathrm{~dB})$ at about $1.2 \mathrm{kHz}$. Whereas females of the ancestral species have a weak preference for calls consisting only of the secondary band to calls having only the dominant band, females of the derived species strongly prefer calls with just the dominant band. Thus, an evolutionary change in preference has preceded any change in the spectral properties of the call. Temporal differences between the species include pulse rate, pulse duration, and pulse shape. Whereas females of the ancestral species use pulse rate alone for call discrimination, females of the derived species base preferences on all three properties, thus suggesting a co-evolutionary change. [Work supported by NSF and NIMH.]

\section{9:00}

3aABa3. Evolution of vocalization behavior in a Madagascar amphibian. Peter M. Narins (Dept. of Physiological Sci., Univ. of California, Los Angeles, 405 Hilgard Ave., Los Angeles, CA 90095) and Edwin R. Lewis (Univ. of California, Berkeley, CA 94720)

Vocalizations of Boophis madagascariensis (Rhacophoridae) males were recorded in a mid-elevation rain forest in eastern Madagascar. Call notes made by males of this species were classified into 28 types. This represents the largest known call repertoire of any amphibian. Even considering all iambic notes as variants of one type, there remain eight distinct call notes produced by this species. Morphological analysis of the vocal musculature of $B$. madagascariensis revealed no obvious adaptations for unusual glottal flexibility or function. Playback studies using a subset of the call notes as stimuli were carried out in the animals' natural habitat to determine the functional significance of several of the call notes. One sequence of notes evoked increased call rates from males, other note sequences inhibited males from calling, whereas others appeared to have no effect on male calling rate. Call note pairs occurred inconsistently and unpredictably. The evolutionary significance of an extended vocal repertoire and a random call note production scheme are discussed. [Work supported by NIDCD Grant Nos. DC-00222 to PMN and DC-00112 to ERL.] 
3aABa4. Moths' ears evolved first for bat defense and later for communication. Annemarie Surlykke (Ctr. for Sound Commun., Inst. of Biol., Odense Univ., DK-5230 Odense M., Denmark, ams@dou.dk)

Many moths possess simple ears primarily to alert them to the echolocation calls of hunting bats. Since large targets reflect stronger echoes than small ones, bats should detect moths with large wing and body surface areas at greater distances than small moths. The increased conspicuousness of large moths would reduce their safety margin over bats, unless this is offset by a greater sensitivity of their ears. Results seem to support this: The sensitivity of ears of noctuid moths is significantly dependent on size, with larger moths possessing lower thresholds. If moth ears exist for bat detection, moths that live in bat-free niches should exibit reduced acoustic sensitivity. Studies of moths temporally isolated from bats (flying either in the winter or during the day) have confirmed this expectation. Another effect of the release of predation pressure from bats may be the use of the preexisting ears for sexual acoustic communication. All sonorous noctuoid moths described so far produce sounds in the same frequency range as that emitted by bats, presumably because bat-sensitive ears are the ancestral (plesiomorphic) condition and acoustic communication is a facility aquired later. [Work supported by the Danish National Research Foundation.]

\title{
Contributed Paper
}

9:40

3aABa5. Bats, insects, and the evolution of hearing. Lee A. Miller (Inst. of Biol., Ctr. for Sound Commun., Odense Univ., DK-5230 Odense M., Denmark)

Insectivorous bats appeared in the fossil record about 50 million years ago, or long after certain insects had adopted nocturnal habits. Many of these nocturnal insect species already had complex ears used mainly for intra-specific communication. Due to their physically small size, insects can produce high-frequency sounds most efficiently. Thus many species were "preadapted" for hearing bats. Examples would be certain beetles and bush crickets. Some nonhearing insects presumably evolved the ca- pacity to hear the ultrasonic signals of bats. One well-documented case is that of the green lacewing, where the first fossil records of ears appeared after bats evolved. Many species of moths have ears tuned to bat signals, an adaptation that has evolved independently in different families and presumably in direct response to bat predation. All evidence points to a substantial selection pressure applied by bats on nocturnal insects. With the preoccupation of hearing mechanisms to avoid predation, other responses of nocturnal insects to echolocating bats might be overlooked. One example could be the advantage for nocturnal insects to occupy the "clutter zone" near or in vegetation, which would mix echoes from the prey with those of clutter. [Work supported by the Danish National Research Foundation.]

WEDNESDAY MORNING, 17 MARCH 1999

ROOM MA001, 10:20 A.M. TO 12:20 P.M.

\section{Session 3aABb}

\section{Animal Bioacoustics: Evolution in Hearing and Sound Production II}

\author{
Darlene R. Ketten, Cochair \\ Department of Biology, Woods Hole Oceanographic Institution, Woods Hole, Massachusetts 02543 \\ Lee A. Miller, Cochair \\ Center for Sound Communication, Institute of Biology, Odense University, DK-5230 Odense M., Denmark
}

Invited Papers

10:20

3aABb1. Temporal resolution in birds and the perception of complex sounds. Robert J. Dooling (Dept. of Psych., Univ. of Maryland, College Park, MD 20742), Marjorie R. Leek (Walter Reed Army Medical Ctr., Washington, DC 20307), and Micheal L. Dent (Univ. of Maryland, College Park, MD 20742)

On many measures of temporal processing, including maximum and minimum temporal integration and duration discrimination, birds show sensitivities similar to those of humans and other mammals. More recent studies on difference limens for short duration tones, discrimination of gaps in sinusoidal markers, and detection of a mistuned harmonic, however, indicate that birds are much more sensitive than humans to certain temporal aspects of complex sounds. Further explorations of auditory temporal analysis by budgerigars (Melopsittacus undulatus) are described here, using sets of harmonic complexes with identical long-term spectra but with temporal waveforms differing either in envelope shape or fine structure. Behavioral discrimination and masking data using these temporal complexes show a high degree of temporal resolving power by the avian auditory system. These findings will be discussed in the context of previous studies on temporal modulation transfer functions and temporal windows in the budgerigar. In aggregate, these results strongly support the long-held belief that birds are more sensitive than humans and other mammals to temporal aspects of complex acoustic signals. [Work supported by NIH DC00198.] 
3aABb2. Audiogram, morphological contraints, environmental acoustics, and adaptation. Wolfgang Plassmann and Evelyn Glaser (Dept. of Zoology, J. W. Goethe Univ., Frankfurt/Main, Germany)

Phylogenetic contraints are a severe restriction on the morphological diversity of the peripheral auditory system in mammals. In order to investigate problems of sensory adaptation under selective pressure, one has first of all to show in a model analysis the potential variance of the system and physiological consequences resulting from these variances. On the basis of these theoretical considerations, morphological investigations, and physiological measurements, an attempted explanation of given auditory systems can be offered based on additional field observations. Following this line of argument, the subsequent topics will be adressed: (a) size and allometric relations of middle and inner ear structures will be presented; (b) consequences of morphological size and changes of allometric relation for the audiogram will be discussed by means of theoretical models and experimental studies; (c) sound propagation properties of different environments such as desert, grassy plain, and forest will be demonstrated. Based on these data, possible selective pressure on the peripheral auditory system in various habitats will be discussed in relation to audiograms, vocalization, and abiotic sound.

\section{$11: 20$}

3aABb3. Acoustic fatheads: Parallel evolution of underwater sound reception mechanisms in dolphins, turtles, and sea birds. Darlene R. Ketten (Woods Hole Oceanogr. Inst., Woods Hole, MA 02543 and Harvard Med. School, Boston, MA 02114, dketten@whoi.edu), Connie Merigo, Elisabeth Chiddick, Howard Krum (New England Aquarium, Boston, MA 02114), and Edward F. Melvin (Univ. of Washington, Seattle, WA 98105)

Dolphins lack conventional external ear canals, seal ear canals vary widely in size and patency, and sound conduction mechanisms in aquatic birds and turtles are virtually unknown. In this study, computerized tomography (CT) and magnetic resonance imaging (MRI) were used to map densities of tissues associated with the outer, middle, and inner ears of three dolphin, two seal, two turtle, and two sea-bird species. Three-dimensional reconstructions of scan data were used to determine species-specific geometry of tissue groups connected to the middle ear or surrounding the ear canal. The analyses show bundles of coherent fatty tissues emerging from the middle ear in all species examined. Densities of these fats are similar across species and are consistent with sound speeds near that of sea water. In seals and birds, these fats sheathed the external auditory canal. In turtles, the fats formed a discrete column communicating with the surface of the head. In dolphins, the fats formed three distinct bundles: two directed anteriorly along the lower jaw with a third projecting laterally. These findings suggest that all four groups evolved parallel soft tissue mechanisms that act as low impedance channels for underwater sound. [Work supported by Mellon Foundation, Seaver Institute, and Office of Naval Research.]

\section{1:40}

3aABb4. The upper frequency range in pigeon hearing: Response properties in auditory nerve and cohlear nucleus-is tuning different in birds? Jean W. Th. Smolders, Marcus Mueller, and Rainer Klinke (Physiologisches Institut III, Theodor-Stern-Kai 7, 60590 Frankfurt am Main, Germany, klinke@em.uni-frankfurt.de)

Information on responses of auditory nerve fibers in birds is generally limited to the low and mid-frequency ranges of hearing, due to anatomical constraints using the standard ganglion cell approach through the opened tympanic recess. Intracranially recorded responses of single units from the auditory nerve and the overlying cochlear nuclei in the pigeon (Columba livia) were compared to those recorded from the ganglion. Characteristic frequencies (CFs) of auditory units ranged from $0.08-5.7 \mathrm{kHz}$ with intracranial compared to $0.06-1.6 \mathrm{kHz}$ with intrascalar recordings. Response properties were qualitatively and quantitatively comparable in the overlapping $\mathrm{CF}$ ranges $(0.08-1.6 \mathrm{kHz})$. Mean spontaneous firing rate decreased monotonically with $\mathrm{CF}$. The $\mathrm{CF}$ thresholds were lowest (0-5 dB SPL) in the CF range 0.6-1.5 kHz. Mean sharpness of tuning $(\mathrm{Q} 10 \mathrm{~dB})$ varied nonmonotonically with $\mathrm{CF}$, with a maximum near $2 \mathrm{kHz}$. In addition, $\mathrm{Q} 10 \mathrm{~dB}$ of pigeon auditory units up to $\mathrm{CFs}$ of $2 \mathrm{kHz}$ was much higher than that of gerbil (Meriones unguiculatus) primary fibers in the same CF range, recorded in the same setup. As it is highly improbable that mechanical tuning in birds would by far exceed that in mammals, this finding is further evidence for a difference in avian and mammalian tuning mechanisms. [Work supported by the DFG, SFB 269.]

\section{Contributed Paper}

\section{2:00}

3aABb5. Adaptations for hearing in background noise. Georg $\mathrm{M}$. Klump, Ulrike Langemann, Ingo Hamann, and Andreas Nieder (Institut fuer Zoologie, Technische Universitaet Muenchen, Lichtenbergstr. 4, D-85748 Garching, Germany, Georg.Klump@bio.tum.de)

Acoustic communication in the natural environment is greatly affected by the ubiquitous background noise. Therefore, vertebrates have evolved mechanisms to efficiently cope with auditory masking. Spectral filtering of the acoustic input is one possibility to improve signal detection. In addition, communication efficiency can be enhanced by exploiting the amplitude fluctuations in background noise. This improvement of signal detection has been termed "comodulation masking release" (CMR). CMR has two components: (1) release from masking within the spectral filter (i.e., a critical band) centered on the signal and (2) release from masking by comparison of correlated amplitude fluctuations (i.e., comodulation) across separate spectral filters. An excellent animal model for studying CMR is the European starling, a songbird exhibiting a temporal resolution and frequency selectivity in its auditory system that is similar to the human's processing performance. Psychoacoustic experiments in starlings show a considerable CMR both within and across spectral filters. Furthermore, the range of envelope frequencies is an important determinant of the amount of CMR. Neurophysiological data recorded in the starling's auditory forebrain reveal that the temporal encoding of the stimulus envelope within auditory filters is a major factor determining the amount of CMR. [Work supported by the DFG, FG Hoerobjekte.] 


\title{
Session 3aAOa
}

\section{Acoustical Oceanography: Innovations in Fish and Plankton Acoustics III (Poster Session)}

\author{
Kenneth G. Foote, Chair \\ Institute of Marine Research, P.O. Box 1870, Nordnes, N-5024, Bergen, Norway
}

\section{Contributed Papers}

\begin{abstract}
All posters will be on display in the Poster Gallery from Monday to Wednesday, 15-17 March. Authors will be at their posters on Wednesday, 17 March. To allow contributors an opportunity to see other posters, contributors of odd-numbered papers will be at their posters from 8:00 a.m. to 10:00 a.m. and contributors of even-numbered papers will be at their posters from 10:00 a.m. to 12:00 noon.
\end{abstract}

\begin{abstract}
3aAOa1. Comparison of two in situ tuna target strength measurement methods. Arnaud Bertrand and Erwan Josse (ORSTOM, BP 70, 29280 Plouzane, France, arnaud.bertrand@orstom.fr)

Two approaches were developed for in situ tuna TS measurement in French Polynesia. One consists of coupling split beam TS measurement with sonic tracking of tagged fish. The other one consists of TS measurement on tuna aggregated close to a Fish Aggregating Device. The first method was used on well-identified fish, swimming freely in their environment and allowing observation of the effect of fish behavior on TS up to $500 \mathrm{~m}$ of depth. The second method was applied on juvenile fish located close to the surface. In this case, fish were not individually identified and a fish sample was caught by trolling line in order to determine the length and species composition of the aggregation. Both methods are complementary and results are similar according to body length.
\end{abstract}

3aAOa2. Modeling the target strength of Meganyctiphanes norvegica. David T. I. Francis (School of Electron. and Elec. Eng., Univ. of Birmingham, Edgbaston, Birmingham B15 2TT, UK, francist@ee-admn.bham.ac.uk), Tor Knutsen, Kenneth G. Foote (Inst. of Marine Res., N-5024 Bergen, Norway), and Lucio Calise (Centro Marino Internazionale, I-09072 Torregrande (OR), Italy)

The euphausiid Meganyctiphanes norvegica is modeled as a fluidlike body having the actual shape of the head and thorax, as reconstructed from dorsal and lateral views recorded with a video camera with macrolens. The mass density has been measured for individual specimens in a densitygradient column, and the longitudinal-wave sound speed in the body, estimated from the measured sound speed through an assemblage of animals in a velocimeter. Theoretical target strength spectra are computed by the boundary element method at a number of discrete frequencies spanning the range $25 \mathrm{kHz}$ to $3.2 \mathrm{MHz}$. [Support by the following is acknowledged: EU through RTD Contract No. MAS3-CT95-0031, Norwegian Research Council through Grant No. 113809/122, and Bergen Large-Scale Facility (LSF) for Marine Pelagic Food Chain Research.]

3aAOa3. Target strength spectra of swim-bladdered fish. Kenneth G. Foote (Inst. of Marine Res., P.O. Box 1870 Nordnes, N-5024 Bergen, Norway) and David T. I. Francis (Univ. of Birmingham, Edgbaston, Birmingham B15 2TT, UK)

Swim-bladdered fish are modeled by the actual shape of the swimbladder as determined from microtomed sections. Target strength spectra are computed for specimens of pollack (Pollachius pollachius) and saithe (Pollachius virens). Results obtained with both the Kirchhoff approximation and boundary-element method at each of four ultrasonic frequencies are compared with each other and with measurements of target strength of the same specimens before swim bladder morphometry. [Partial support of the EU through RTD Contract No. MAS3-CT95-0031 is acknowledged.]

3aAOa4. Broad-scale volume backscattering strength measurements in the Black Sea. Erhan Mutlu, Ali C. Gucu, and Ferit Bingel (Inst. of Marine Sci., P.O. Box. 28, 33731, Erdemli, Icel, Turkey)

Enhanced echograms of high-frequency sound (120 and $200 \mathrm{kHz})$ from entire tracklines surveyed during summer and winter of 1991-1994 were evaluated to observe volume backscattering in the Black Sea. Echo intensities were filtered through two thresholds for distinguishing suspended scatterers from active swimmers (fish). It was postulated that the backscattering by the suspended matters was associated with bathymetry, seasonal stratification and different levels of mixing of water, and daily migration of zooplankton. The scattering was layered in association with the physical structure in the open water. As bottom depth was shoaled, the vertical structure became more homogenous. Biological scatterers were diversified with zooplanktoners and pelagic fish (anchovy and sprat). Copepod and gelatinous organisms (comb-jelly and moon jellyfish) appeared to be responsible for the zooplanktonic scatterers. Changes in seasonal and diurnal migrational patterns of fish were investigated. During the summer, pelagic fish schools were observed in an offshore area, whereas in winter the schools were confined to the coastal area. In regard to the schooling density, higher concentrations were found in winter.

3aAOa5. Wavelet decompositions versus singular value decomposition (SVD) in a fish target strength estimation. Marek Moszynski and Andrzej Stepnowski (Dept. of Acoust., Tech. Univ. of Gdansk, Narutowicza 11/12, 80-952 Gdansk, Poland, marmo@pg.gda.pl)

The fish target strength estimation when using acoustic echoes from a single-beam echosounder is possible after solving ill-conditioned equations where probability density functions (PDFs) of echo level, target strength, and beam pattern are involved. The modified singular value decomposition (SVD) algorithms are typically used in such cases. Unfortunately, this technique often leads to artifactual modes in solution due to Fourier nature of underlying eigenfunctions. The expectation maximization smoothing (EMS) method is used to improve smoothness of solution but by the cost of increasing the time of computation of estimates. D. L. Donoho (1995) proposed the wavelet-vaguelette decomposition (WVD) as 
being well suited for solving ill-conditioned equations with inhomogeneous functions. This concept was extended to fish target strength estimation and obtained results were compared to SVD performances.

3aAOa6. Gas cavities in phytoplankton. Dmitry A. Selivanovsky, Igor N. Didenkulov (Inst. of Appl. Phys., 46 Ulyanov St., Nizhny Novgorod, 603600, Russia), and Pavel A. Stunzhas (Shirshov's Inst. of Oceanology, Moscow, 117851, Russia)

Acoustical and related properties of phytoplankton suspensions were investigated in laboratory conditions and in situ. A review of studies is present. It was found that cells have gas cavities which allow them to keep a neutral buoyancy. Under sharp compression gas cavities dissolve and recover only under the light exposition. Such properties can explain an anomalous migration of some sound scattering layers. The use of the compression/decompression method allowed us to find gas cavities in blood erythrocites also. The $6.5-\mathrm{MHz}$ acoustic backscattering from phytoplankton suspensions was applied for characterization of reverberation level under the natural condition and compression (up to $5 \mathrm{~atm}$ ), which allowed us to evaluate a relative volume of gas cavities in cells. Gas cavities increase an acoustical cross section by $2-3$ orders. A dispersion of sound velocity in phytoplankton suspensions with the phase and the resonance methods (from $200 \mathrm{~Hz}$ to $11 \mathrm{MHz}$ ) for six kinds of living cell suspensions was also studied. Both the increase and decrease of sound velocity dispersion with frequency was observed. The effect is different for cells of different kinds. [Work supported in part by RFBR, Russia.]

3aAOa7. Modeling considerations for acoustic scattering by fluidlike elongated zooplankton: Model intercomparisons and recommendations for euphausiids and copepods. Timothy K. Stanton and Dezhang Chu (Dept. of Appl. Ocean Phys. and Eng., Woods Hole Oceanogr. Inst., Woods Hole, MA 02543-1053)

Predictions of acoustic scattering by fluidlike elongated zooplankton are made using the deformed cylinder model and Distorted Wave Born Approximation over a wide range of animal size, shape, material property inhomogeneities, and orientation, as well as acoustic frequency. Each shape represents a digitized form of the shape of a euphausiid or copepod at low-, medium-, and high-resolution sampling of the shape (e.g., at low resolution, a straight smooth finite cylinder is used and at high resolution, a rough tapered bent finite cylinder is used). The results are analyzed in order to determine the conditions under which the various degrees of complexity in modeling the scattering are required. For example, the predictions illustrate that for modeling the scattering for animals near broadside incidence, relatively simple models can be used (smooth homogeneous bodies). However, for animals off broadside, which can be the case for downward-looking echosounders insonifying euphausiids and copepods, roughness and sometimes inhomogeneities must be used to model the scattering accurately (roughness effects dominate scattering well off broadside). Comparisons are made with laboratory data involving euphausiids, shrimp, and copepods. [Work supported by ONR.]

3aAOa8. Inference of material properties of zooplankton from acoustic and conductivity measurements. Dezhang Chu (Dept. of Appl. Ocean Phys. and Eng., Woods Hole Oceanogr. Inst., Woods Hole, MA 02543) and Peter Wiebe (Woods Hole Oceanogr. Inst., Woods Hole, MA 02543)

In order to infer correctly biological information from raw acoustic data, various acoustical models have been developed and much progress has been made both in laboratory experiments and field applications. However, the acoustic properties of biological scatterers, such as soundspeed and density, have a profound influence on the target strength estimate or volume scattering strength. A $1 \%-2 \%$ variation in sound speed and density can lead to an error in target strength prediction by as much as $20 \mathrm{~dB}$ or a tenfold bias in biomass estimate, which could be much greater than that due to the choice of different acoustic models. There is a dearth of information about the material properties of the live zooplankton because measurement of them is difficult. An instrumented chamber was used to collect simultaneously acoustic and conductivity data with and without the presence of live zooplankton. The acoustic measurement characterizes both the back- and forward-scattering patterns, while the conductivity measurement provides an estimate of the fraction of volume the animals occupy (volume fraction). The combined information can then be used to infer the material properties of the animal. The experimental results will be presented and discussed. [Work supported by NSF.]

3aAOa9. Digital transducer, novel technology for acoustical oceanography and fisheries research. William Acker, Dan Wiggins (BioSonics, Inc., 4027 Leary Way NW, Seattle, WA 98107, bio@biosonicsinc.com), and Janusz Burczynski (BioSonics Europe, 54036 Marina di Carrara (MS), Italy)

Novel digital transducer architecture designed and produced by BioSonics Inc. offers many advantages over older analog designs. By digitizing the signal at the transducer element, the cable-coupled noise is eliminated. The signal is sampled at a high rate and accuracy $(0.13 \%)$, and wide instantaneous dynamic ranges $(132 \mathrm{~dB})$. This allows simultaneous capture of extremely small and large echoes (single plankton specimen or bottom return) with no loss in detail or signal saturation. The data acquisition system is virtually automatic since most of the decisions are done by software. Row data is stored on PC hard drive. Data acquisition and data analysis software packages are user friendly and operate in Windows ${ }^{\mathrm{TM}}$ environment. Many data processing tools are already developed as separate implementaiton of various algorithms. Digital transducers have already been successfully applied in various field projects in both marine and fresh waters: (i) monitoring and estimates of fish and plankton, (ii) fish tracking, and (iii) bottom plants estimate and seabed classification. The digital sonar is available in various packages and it can be installed on different platforms as portable systems for boats and ships, fixed platforms on dams, and in river in systems. The digital transducers are available in a wide range of frequencies ( $38 \mathrm{kHz}$ to $1 \mathrm{MHz}$ ), in a single, dual, or split beam configurations.

3aAOa10. Spheres for calibrating high-frequency broadband echo sounders. Kenneth G. Foote (Inst. of Marine Res., P.O. Box 1870 Nordnes, N-5024 Bergen, Norway), David T. I. Francis (Univ. of Birmingham, Birmingham B15 2TT, UK), Hilde Furset, and Halvor Hobaek (Univ. of Bergen, N-5007 Bergen, Norway)

The notion of standard-sphere broadband calibration [Dragonette et al., J. Acoust. Soc. Am. 69, 1186 (1981)] is being realized for a new echo sounding system that spans the seven-octave range $25 \mathrm{kHz}$ to 3.2 MHz. Spheres formed of tungsten carbide with $6 \%$ cobalt binder, with 10and 20-mm diameters, have been measured in the laboratory to determine their backscattering spectra over the approximate frequency ranges $0.85-$ 1.3 $\mathrm{MHz}$ and 2.9-3.6 MHz. This allows exploration of the wave number radius (ka) product over the nominal ranges $18-28,36-56,61-75$, and 122-150. Comparison with theoretical expectation, as derived from the standard modal solution using published values for the material properties, is quite good. Confidence in the computations thus enables favorable regions of the backscattering spectra to be sought and exploited in choosing optimal diameters, as has earlier been the case with spheres for calibrating resonant transducers. In the course of checking computations, it was discovered that two independently developed numerical codes yield values 
for the backscattering cross section that agree to within 1 part in 10000 to better than one part in 1000000 for ka of the order of 100. [Partial support of the EU through RTD Contract No. MAS3-CT95-0031 is acknowledged.]

3aAOa11. Shark and salmon movement measured by tracking radartype acoustic transducers. John Hedgepeth, David Fuhriman (BioSonics, Inc., Seattle, WA), Robert Johnson, David Geist (Battelle Memorial Inst., Richland, WA), Norm Bartoo, David Holts (Natl. Marine Fisheries Service, La Jolla, CA), Tim Mulligan, and George Cronkite (Dept. of Fisheries and Oceans, Nanaimo, BC, Canada)

Studies of fish behavior have used a methodology called the tracking transducer. The principle of tracking radar, aligning the antenna beam with a target, was applied with an acoustic split-beam transducer and dual-axis rotators for tracking individual fish over long periods of time. Deviation of the target from the beam axis produces a correction to point the axis toward the target. Initial studies with active acoustics have evolved an acoustic tag tracking method that is proposed for tracking both juvenile salmon and pelagic sharks. The major advance is that active and passive radar-type tracking can be combined in the same instrument. The tracking transducer was first used at Ice Harbor Dam, Snake River, 1995, and in 1996, at The Dalles Dam, Columbia River. Two tracking systems were used to triangulate a small acoustic transmitter in salmonid fish at Lower Granite Dam on the Snake River, Washington. Recently, adult salmon, returning to the Fraser River, were tracked to measure avoidance to surveying vessels. The feasibility for tracking sharks was shown at the Tacoma, Washington Point Defiance Aquarium. A proposed method of simultaneously tracking sharks with echoes and using acoustic tags will allow behavior, abundance, and associated pelagic assemblages to be determined.

3aAOa12. Mechanoreception for food fall detection in deep sea scavengers. Michael Klages (Alfred-Wegener-Inst. for Polar- and Marineresearch Postfach 1201 61, 27515 Bremerhaven, Germany) and Sergey I. Muyakshin (Inst. of Appl. Phys., Nizhny Novgorod, 603600, Russia)

Although knowledge about functional principles of deep-sea ecosystems is rather scarce, it is assumed that the energy supply for scavengers is restricted to large food falls of dead vertebrates. It is generally accepted that chemoreception is one of the major tools for marine organisms to detect food sources. However, another major source of information may come from hydroacoustical feeding noises produced by scavengers appearing on a cadaver reached the seafloor. The aim of the present study was to investigate whether scavenging crustaceans-pandalid shrimps Pandalus borealis - are able to detect such rare food fall events via mechanoreception or not. These results are based on 228 single experiments indicating that these animals possess the sensitivity to the particle dis- placement of $0.1-10 \mathrm{mkm}$ in frequency range $30-250 \mathrm{~Hz}$. Therefore, acoustic feeding noises offer a possibility for animals to detect such rare events but only at distances of a few meters. At such small distances chemoreception is presumably more important. However, based on theoretical calculations on the relevance of various types of waves, originating on the water-sediment interface from any object falling on the seafloor, it is proposed that such "micro seismic events" may allow resting scavengers even some hundred meters away the detection of this event, most likely followed by chemoreceptive tracking.

3aAOa13. Echogram noise quantification with application to herring observations. Rolf J. Korneliussen (Inst. of Marine Res., P.O. Box 1070, Nordnes, N-5024 Bergen, Norway, rolf@imr.no)

Pushing the limits of scientific echo sounders involves considerations of noise, which is inherently frequency dependent. Suprisingly, perhaps, there is also a dependence on bottom depth. In this work, noise is quantified by measurement for a standard echo sounder, the EK500, at 18, 38, 120 , and $200 \mathrm{kHz}$. Use of empirical relations of noise as a function of range to reduce echogram noise is described in general, and illustrated in particular for data collected on Norwegian spring-spawning herring (Clupea harengus) when wintering in the Vestfjord system. [Work supported by the Norwegian Research Council through Grant No. 113517/120.]

3aAOa14. Preliminary description of swimming activity and estimation of swimming speed of saithe (Pollachius virens) at one location in the North Sea. Jens Pedersen (Danish Inst. for Fisheries Res., North Sea Ctr., P.O. Box 101, DK-9850 Hirtshals, Denmark, jp@dfu.min.dk)

Individual saithe were tracked with a split-beam echosounder, while the vessel was drifting, in the area around Eigersundbank in the North Sea and their swimming speed estimated. The average swimming speed was approximately 4 and 1 body lengths per second for small saithe (20-30 $\mathrm{cm}$ ) and saithe $>70 \mathrm{~cm}$, respectively, and a significant inverse relationship between length of the saithe and swimming speed was found. There was clear evidence of diurnal variation in swimming speed of small saithe, as the swimming speed was significantly higher during night (18-06 h) than during day $(06-18 \mathrm{~h})$. The number of observations on saithe $>70 \mathrm{~cm}$ was too small to compare day and night swimming speeds. Although the results indicate higher swimming speeds of saithe in the demersal layer compared to pelagic saithe, significant differences were not found. The duration of acoustic observation time was 36-56 min per 4-h sampling interval during the $24-\mathrm{h}$ cycle. A total of 278 series of saithe were selected, which, according to selection criteria, were accepted as representing tracking of single fish over two pings or more. The species identify of the targets tracked acoustically was verified by trawling in the layers investigated. 


\title{
Session 3aAOb
}

\section{Acoustical Oceanography and Underwater Acoustics: Acoustic Tomography and Thermometry I}

\author{
Uwe Send, Cochair \\ Institut für Meereskunde, University of Kiel, Düsternbrooker Weg 20, 24105 Kiel, Germany \\ Robert C. Spindel, Cochair \\ Applied Physics Laboratory, University of Washington, Seattle, Washington 98105, USA
}

Invited Papers

8:00

3aAOb1. Meddies and front detection by acoustic tomography. Fabienne Gaillard (Laboratoire de physique des océansIFREMER, BP 70, 29280, Plouzané, France, fabienne.gaillard@ifremer.fr)

The CAMBIOS experiment was defined within the CANIGO project as an observing array for the Canary basin. A pair of 400-H acoustic tranceivers set up in the middle of the basin provided a 9-month reciprocal series of arrival amplitude versus time sequences, starting in July 1997 and ending in March 1998. In the Canary basin the temperature and current field are perturbed in the upper layers by the meandering and branching of the Azores front, and at mid-depth by the transit of warm and salty coherent structures called meddies. The first analysis of the acoustic measurements will be presented here, with particular attention dedicated to the signature of the front and meddies, both on direct and reciprocal travel times. [Work supported by the MAST III programme under Contract No. MAS3-CT96-0060.]

8:20

3aAOb2. Resonant diurnal internal tides in the North Atlantic. Brian D. Dushaw (Appl. Phys. Lab., College of Ocean and Fisheries Sci., Univ. of Washington, Seattle, WA 98105) and Peter F. Worcester (Univ. of California at San Diego, La Jolla, CA 92093)

Using an acoustic tomography array located midway between Puerto Rico and Bermuda, enhanced diurnal tidal signals associated with the lowest internal-wave mode have been observed. These signals result from a diurnal internal wave with $O(500-\mathrm{km})$ wavelength that is resonantly trapped between the shelf just north of Puerto Rico and the turning latitude, a distance of about $1100 \mathrm{~km}$. The data obtained using the large acoustical array are consistent with the predicted Airy-function variation with latitude of diurnal internal waves near the turning latitude. The existence of this wave is a striking demonstration of the long spatial and temporal coherence of oceanic internal tides.

\section{8:40}

3aAOb3. Acoustic monitoring of the transport and temperature variability in the Strait of Gibraltar. Peter F. Worcester (Scripps Inst. of Oceanogr., Univ. of California at San Diego, La Jolla, CA 92093-0225, pworcester@ucsd.edu), Uwe Send (Univ. of Kiel, 24105 Kiel, Germany), Bruce D. Cornuelle, and Christopher O. Tiemann (Univ. of California at San Diego, La Jolla, CA 92093)

Three acoustic transceivers transmitted across the Strait of Gibraltar during April-May 1996 to determine the feasibility of using acoustic methods to measure the transport and average temperature in the Strait. The first arrivals, from ray paths confined to the (lower layer) Mediterranean water, give sum travel times that are remarkably stable, with a slow peak-to-peak variation of only about $3 \mathrm{~ms}$ over a two-week period, corresponding to an integrated temperature change of about $70 \mathrm{~m}{ }^{\circ} \mathrm{C}$. The difference travel times from a path diagonal to the axis of the Strait show a peak-to-peak tidal variation of about $\pm 5 \mathrm{~ms}$, corresponding to integrated currents parallel to the acoustic path of about $\pm 50 \mathrm{~cm} / \mathrm{s}$, which is consistent with estimates derived from a moored current meter array. One of the transceivers also had a two-element horizontal receiving array, to determine the feasibility of using arrival angle to measure the current perpendicular to the acoustic path. The measured phase differences are encouragingly similar to phase differences predicted using the current meter data. Later arrivals, from ray paths that sampled the (upper layer) Atlantic water, are much more complicated, in part due to the effects of the internal wave bores present in the Strait. 


\section{Contributed Papers}

3aAOb4. AMOC: Acoustic monitoring of the ocean climate of the Arctic. Ola M. Johannessen and AMOC Group (NERSC, Edv. Griegsvei 3A, 5037 Solheimsviken, Norway and Geophysical Inst., Univ. of Bergen, Bergen, Norway, hanne.sagen@nrsc.no)

The overall objective of AMOC is to develop and design an acoustic system for long-term monitoring of the ocean temperature and ice thickness in the Arctic Ocean, including the Fram Strait, for climate variability studies and global warming detection. The unique combination of the underwater acoustic remote sensing with satellite remote sensing of the ice cover including modeling and data assimilation, in the predicted sensitive climate region of the Arctic Ocean, is perhaps the key solution to monitor global climate changes and early detection of global warming. AMOC has the following specific objectives: (i) Compilation and analysis of existing ocean and ice data from the Arctic Ocean. (ii) Simulation of present and future Arctic Ocean environment to be used as input to acoustic modeling. (iii) Simulation of present and future basin-wide acoustic propagation using natural variability and global warming scenarios to investigate the sensitivity of acoustic methods for global warming detection. (iv) Simulation of present and future acoustic propagation in the Fram Strait to investigate the sensitivity of acoustic methods for monitoring heat and volume fluxes in an area of strong mesoscale eddy activity. (v) Design of an optimal acoustic monitoring system for climate change detection in the Arctic.

\section{9:20}

3aAOb5. Sound-signal scintillation approach to acoustic monitoring of the heat and mass transport through the Fram Strait. Konstantin A. Naugolnykh (CIRES, Univ. of Colorado/ETL, NOAA, Boulder, CO 80303), Igor B. Esipov, Oleg B. Ovchinnikov, Yuri I. Tuzhilkin (N. Andreev Acoust. Inst., Moscow, 117036, Russia), and Ola M. Johannessen (Univ. of Bergen, Bergen, Norway)

The computation model of the sound-signal propagation in the Fram Strait environment is considered in application to remote acoustic sensing of the heat and mass transport. A 3-D sound-speed environment has been simulated with respect to the statistical features of the real cross section of the strait obtained from experimental data. The linear four-element transmission array and four-element receiving arrray with equally spaced elements are modeled. Travel time variations of the signals propagating across the strait related to temperature change are calculated and the methods of the temperature retrieval in the complex environment is discussed. Then the travel time perturbations of the sound signals produced by the advection of the small-scale inhomogeneities across the path are considered. The calculation of the time-lagged cross-correlation function of the acoustic field gives the transverse current velocity data. By combining the response from each transmitter-receiver pair in different ways, the transverse current profile is retrieved. Results obtained are used to estimate the potential accuracy of the acoustic sensing of the heat and mass transport through the strait cross section. [Work supported by Environmental and Climate Programme of EC, Project PL970338.]

\section{$9: 40$}

3aAOb6. AMOC: Listen to the climate change? Hanne Sagen (NERSC, Edv. Griegsvei 3A, 5037 Solheimsviken, Norway, hanne.sagen@nrsc.no) and Ola M. Johannessen (Univ. of Bergen, Bergen, Norway)

Acoustical methods such as acoustic tomography and thermometry have proved their importance in future long-term monitoring of ocean climate. The scope of this presentation is to show how ambient noise can be used as additional information in a future acoustic monitoring system for the Arctic ocean. To our knowledge, no inversion of ambient noise characteristics to sea ice and ocean parameters has been attempted neither in the MIZ nor the interior of the Arctic Ocean. Key parameters in ocean climate monitoring in the Arctic are averaged ice thickness and averaged ocean temperature profiles. These parameters are very difficult and time demanding to measure by using point measurements, and unavailable using electromagnetic waves. Therefore, special attention has been paid to developing new concepts for measuring ocean stratification and averaged ice thickness. A new concept combining wave models, SAR data, and ambient noise recordings for retrieving the sea ice thickness and elastic parameters of the sea ice in MIZ are presented. Another concept is proposed for use in the MIZ and in the interior Arctic using averaged ambient noise frequency spectra to obtain information about the sea ice and ocean stratification.

\section{0:00-10:20 Break}

\section{Invited Paper}

10:20

3aAOb7. Acoustic studies of the large-scale ocean circulation. Dimitris Menemenlis (Jet Propulsion Lab., California Inst. of Technol., MS 300-323, 4800 Oak Grove Dr., Pasadena, CA 91109, dimitri@pacific.jpl.nasa.gov)

Detailed knowledge of ocean circulation and its transport properties is prerequisite to an understanding of the earth's climate and of important biological and chemical cycles. Results from two recent experiments, THETIS-2 in the Western Mediterranean and ATOC in the North Pacific, illustrate the use of ocean acoustic tomography for studies of the large-scale circulation. The attraction of acoustic tomography is its ability to sample and average the large-scale oceanic thermal structure, synoptically, along several sections, and at regular intervals. In both studies, the acoustic data are compared to, and then combined with, general circulation models, meteorological analyses, satellite altimetry, and direct measurements from ships. Both studies provide complete regional descriptions of the time-evolving, three-dimensional, large-scale circulation, albeit with large uncertainties. The studies raise serious issues about existing ocean observing capability and provide guidelines for future efforts.

\section{Contributed Papers}

\section{0:40}

3aAOb8. ATOC or Candide: Toward depth-averaged temperature maps of the North Pacific Ocean. Brian D. Dushaw and the ATOC Group $^{\text {a) }}$ (Appl. Phys. Lab., College of Ocean and Fisheries Sci., Univ. of Washington, Seattle, WA 98105)

The ATOC Project has aquired trans-Pacific acoustic data from two acoustic sources: one located on Pioneer Seamount off the coast of California, the other located north of Kauai, Hawaii. Transmissions from these sources are detected by U.S. Navy SOSUS arrays located throughout the North Pacific. The time series of acoustic travel times from the Pioneer Seamount and Kauai transmissions are about $1 \frac{1}{2}$ and 1 year long, respectively. Both time series have highly irregular sampling because of marine mammal protocols and temporary cable failures. These acoustic data are used to estimate ocean temperature accurately. At the time of writing this abstract, however, the overlap of the two time series is just beginning. The overlapping time series will for the first time allow estimates of temperature maps of the North Pacific basin using long-range acoustics because the acoustic paths from these sources criss-cross the North Pacific. This 
talk will show the initial maps of depth-averaged temperature of the North Pacific Ocean derived using the time series to be collected through Fall 1998. ${ }^{\text {a) }}$ The Acoust. Thermometry of Ocean Climate (ATOC) Group is: A. B. Baggeroer, D. Menememlis [now at JPL], and C. Wunsch (MIT); T. G. Birdsall, K. Metzger (Univ. of Michigan); C. Clark (Cornell Univ.); J. A. Colosi (WHOI); B. D. Cornuelle, M. Dzieciuch [now at ABMF], W. Munk, P. F. Worcester (SIO); D. Costa (Univ. of California, Santa Cruz); B. D. Dushaw, B. M. Howe, J. A. Mercer, R. C. Spindel (APL-UW); A. M. G. Forbes (CSIRO, Hobart).

\section{1:00}

3aAOb9. Measurements of multi-megameter travel-time bias and coherence. M. Dzieciuch, P. Worcester (Scripps Inst. of Oceanogr., Univ. of California, San Diego, CA), T. Birdsall, K. Metzger (Univ. of Michigan), B. Howe, J. Mercer, and R. Spindel (Univ. of Washington)

The quality of ocean acoustic travel-time measurements depends on the coherence as well as the bandwidth of the signal. Ocean internal-wave fields are thought to be responsible for the loss of coherence in lowfrequency acoustic signals. If the coherence bandwidth is less than the signal bandwidth, it is possible to consider sub-bands of the signal as separate measurements. The separate measurements can then be combined incoherently to improve the quality of the travel-time measurement. Theoretical work and computer simulations also predict that the travel-time of acoustic signals is biased by the ocean internal wave field. The pathintegral theory for scattering predicts that the size of the travel-time bias depends on the logarithm of the center frequency of the acoustic signal, thus separate sub-bands would have different travel times and incoherent recombination would not be an optimal procedure. A recent experiment, in which two acoustic signals with different center frequencies were transmitted simultaneously, makes it possible to measure the bias experimentally and to determine the efficiency of sub-band averaging. Preliminary results suggest that the bias is about $50 \mathrm{~ms}$ at $3500-\mathrm{km}$ range, which is roughly as large as expected.

\section{1:20}

3aAOb10. Reciprocal tomography between ATOC transmitters. James A. Mercer, Brian Dushaw, Bruce M. Howe, Robert C. Spindel (Appl. Phys. Lab., Univ. of Washington, Seattle, WA 98105), Peter F. Worcester (Univ. of California at San Diego, La Jolla, CA 92093), and Kurt Metzger (Univ. of Michigan, Ann Arbor, MI 48109)

The Acoustic Thermometry of Ocean Climate (ATOC) sound sources are located 8 miles north of Kauai, Hawaii and 50 miles west of Half Moon Bay, California. The sources are connected to shore-based electronics by undersea coaxial cables. Recent modifications in the shore-based electronics have permitted the ATOC transducers to serve as hydrophones as well as transmitters. Data collected over this $4-\mathrm{Mm}$ reciprocal path will be presented and discussed in terms of current and internal wave effects. [Work supported by ONR.]

\section{1:40}

3aAOb11. Modal structure of low-frequency, broadband receptions at megameter ranges. Kathleen E. Wage, Arthur B. Baggeroer (Res. Lab. of Electron., MIT and Woods Hole Oceanogr. Inst., Woods Hole, MA 02543), and James C. Preisig (Woods Hole Oceanogr. Inst., Woods Hole, MA 02543)

Data from the Acoustic Thermometry of Ocean Climate (ATOC) experiment show that at $3515-\mathrm{km}$ range, each low (axial) mode signal contains a series of arrivals, rather than a single dispersive arrival, which would characterize adiabatic propagation. Short-time Fourier transform (STFT) analysis demonstrates that this multipath structure exhibits frequency-selective fading and significant temporal variability. Despite the complexity of individual receptions, averaging the mode estimates for transmissions at successive four-hour intervals reveals some of the expected dispersion characteristics of a deep water channel. Although this evidence suggests that the modes retain travel-time information at megameter ranges, the complicated nature of the arrivals indicates that a better understanding of mode coupling due to internal waves is required for tomography and matched-field processing applications. This study investigates the temporal and frequency coherence of broadband modes by comparing PE simulations with the ATOC results. Since ambient noise and environmental uncertainty affect the accuracy of mode signal measurements for experimental data, this research also addresses certain practical issues and limitations involved in estimating mode coherence. [Work supported by ONR.]

\section{2:00}

3aAOb12. On the existence of nearly stable dynamics in long-range sound propagation through oceanic mesoscale structure. Michael A. Wolfson and Steven Tomsovic (Dept. of Phys., Washington State Univ., Pullman, WA 99164-2814, mwolfson@wsu.edu)

A dynamical system, motivated by ocean acoustic pulse propagation in the horizontal plane along the sound channel axis, is constructed using a potential derived from an idealized mesoscale eddy field. This problem is known to exhibit chaotic behavior in that rays are exponentially sensitive to both their initial condition and the position and strength of the individual eddies. The measure of chaos for the system has been expressed by deriving a Lyapunov exponent which determines the mean growth rate for the envelope of one of the elements of the stability matrix [M. A. Wolfson and F. Tappert, J. Acoust. Soc. Am. (to be published, 1998)]. However, the individual stability matrix elements are seen to fluctuate wildly from one ray to another. It turns out that many rays exhibit absolute traces of the stability matrix with values of $O(1)$ even at basin scale ranges. This implies that much of the dynamics involves only local rotations as the flow evolves in phase space. Estimates are presented for a range-dependent measure of this nearly stable dynamical feature. Its relation to tomographic inversions of some features of mesoscale eddy fields is discussed. 


\title{
Session 3aBB
}

\section{Biomedical Ultrasound/Bioresponse to Vibration: HIFU and Thermal Effects}

\author{
Ronald A. Roy, Cochair \\ Department of Aerospace and Mechanical Engineering, Boston University, Boston, Massachusetts 02215, USA \\ Ibrahim Hallaj, Cochair \\ Applied Physics Laboratory, University of Washington, Seattle, Washington 98105, USA
}

Contributed Papers

8:00

\begin{abstract}
3aBB1. Numerical simulation of the enhanced heat production in tissue due to the nonlinear character of high-intensity focused ultrasound (HIFU). Siegfried Ginter, Eckard Steiger, and Rainer Riedlinger (Inst. fuer Hoechstfrequenztechnik und Elektronik/Akustik, Univ. Karlsruhe, D-76128 Karlsruhe, Germany)
\end{abstract}

HIFU is used in thermotherapy to destroy tumors located deep in human tissue by heat. It is well known that HIFU is connected with the generation of higher-frequency components due to nonlinear steepening during propagation. An over-proportional heat generation in tissue occurs with enhanced power input. To obtain fundamental information about an optimal dosage of the applied ultrasound, simulations are necessary. A hot spot in healthy tissue must be avoided and a sufficient heat delivery in the tumor region must be guaranteed. Until now most numerical investigations of thermotherapy with HIFU have been carried out under the assumption of linearity. This means an essential underestimation of heat production. Here an improved model is introduced which enables accurate simulations, including nonlinearities. It consists of a set of nonlinear acoustic equations which ensure an accurate full-wave propagation modeling. A broadband absorption model of typical soft-tissue frequency-power-law character is included to obtain a nonlinear modeled heat source. The thermal behavior is described by a bio-heat-transfers-equation. The complete model is solved by means of a high-order FDTD scheme. Exemplary simulations illustrate the efficiency of this method.

\section{8:20}

3aBB2. Hyperthermia therapy using phase conjugation. Philippe Roux, Michael B. Porter, Hee C. Song, and William A. Kuperman (Marine Physical Lab., Scripps Inst. of Oceanogr., UCSD, 9500 Gilman Dr., La Jolla, CA 92093-0238)

One of the advantages of ultrasound phased-array treatments is the noninvasive manner in which ultrasonic energy, and then heat, can be delivered to the treatment volume. However, the heat generated in the medium induces variations in the sound speed, which affects the acoustic focusing and leads to strong effects such as self-focusing or selfdefocusing of the sound beam. In this work, a numerical simulation is used to study coupled acoustic-heat propagation in tissue. By simultaneously modeling the propagation of acoustic waves and the diffusion of heat, it was possible to simulate quantitatively acoustic refraction induced by heat in the treatment volume. Finally, using phase conjugation, one procedure to control temperature and maintain the acoustic focusing at the initially desired focal point was developed.
$8: 40$

3aBB3. Diagnostic ultrasound for two-dimensional temperature mapping in hyperthermia and laser-induced tissue coagulation. Hendrik Willig, Annette Pfennig, Volker Haenel, Bernhard Kleffner (Fraunhofer Inst. for Biomed. Eng., Ultrasound Dept., Ensheimer Str. 48, D-66386 St. Ingbert, Germany, willig@ibmt.fhg.de), and Karsten Schwerdtfeger (Universitaet des Saarlandes, Universitaetskliniken, 66421 Homburg, Germany)

Laser-induced tissue heating and coagulation is a common technique in minimally invasive surgery to achieve localized thermal tissue damage. Precision and efficiency of treatment could be improved markedly by noninvasive spatio-temporal temperature monitoring. A method for twodimensional mapping of tissue temperature as well as tissue structural changes has been investigated in vitro. The algorithms are based on the temperature dependence of speed of sound and a combination of additional acoustical parameters in order to consider the expansion of the heated volume, creation of gas bubbles, and carbonization. An approach for motion compensation, as it is necessary for clinical application, will be described in short.

\section{9:00}

3aBB4. Noninvasive estimation of tissue temperature via ultrasound frequency shift using a narrow-band transducer. Seong-Wook Song, Koeng-Mo Sung (School of Elec. Eng., Seoul Natl. Univ., Seoul, Korea), Min-Joo Choi (Cheju Natl. Univ.), and Jun-Seok Lim (Sejong Univ.)

When applying the high power focused ultrasound to medical therapy, it is necessary to monitor the temperature change in tissue. Some methods have been proposed to measure this change noninvasively. One of those techniques measures the frequency changes in harmonics which are linear with the temperature changes. The linear coefficient has been defined with the fundamental frequency changes and the temperature changes. This measurement requires at least three harmonics, so that it requires a wideband transducer to estimate the fundamental frequency efficiently. This paper suggests a method to measure the temperature changes noninvasively with only one component of harmonics, under the condition that the proportional constant is defined such as the thermal expansion coefficient. It does not require the wideband transducer. Additionally, it shows the mutual relationship between thermal expansion coefficient, thermal frequency shift coefficient, and thermal speed change coefficient, which are defined in similar form.

9:20

3aBB5. Thermal effect of ultrasound enhancer for transdermal insulin delivery. E. J. Park and S. W. Yoon (Dept. of Phys., Sung Kyun Kwan Univ., 300 Chunchundong, Suwon 440-746, Kyungkido, Republic of Korea, swyoon@yurim.skku.ac.kr)

Ultrasound has been known as a good enhancer for transdermal drug delivery. However, major mechanisms in the ultrasound enhancer to improve the transdermal drug delivery were not well understood. In this 
study a simple experiment was designed to simulate the human skin system with rat back skin. An unfocussed 1-MHz ultrasound transducer and a heating wire loop were used as enhancers. Transmitted amounts of insulin with the ultrasound enhancer were increased ten times more than those without any enhancer. The ultrasound enhancer also improved by $25 \%$ the enhancement of insulin transportation compared with that due to thermal effect solely by heating wire loop. It was also observed that rapid temperature increase with ultrasound more efficiently enhanced transdermal transport of insulin. The thermal effect of the ultrasound enhancer is suggested as a most likely mechanism for transdermal insulin delivery.

\section{9:40-10:00 Break}

\section{0:00}

3aBB6. Sound scattering and local heating from pulse-driven microbubbles. Sascha Hilgenfeldt (DEAS, Harvard Univ., 29 Oxford St., Cambridge, MA 02138, sascha@stokes.harvard.edu), Detlef Lohse (Univ. of Twente, 7500 AE Enschede, The Netherlands), and Michael Zomack (Schering AG, Clinical Development, 13342 Berlin, Germany)

Using a bubble dynamical model based on the Rayleigh-Plesset equation, it is shown that the experimentally observed spectra of sound emitted from diagnostic microbubble contrast agents under the action of short, strong ultrasonic pulses can be understood from the interplay of enhanced sound emission due to nonlinear bubble oscillations and enhanced sound damping due to the occurrence of high frequencies in the emitted spectrum. The latter effect counteracts the pronounced increases in the scattering cross sections in the regime of nonlinear bubble response. Sound absorption furthermore leads to highly localized heating of the liquid (e.g., blood) over a distance of several $\mu \mathrm{m}$ near the collapsing bubble. This temperature rise is, however, too brief and the heated liquid volume too small to be hazardous to the patient's health.

\section{0:20}

3aBB7. Numerical modeling of high-intensity focused ultrasound for hemostatic applications. Vera A. Khokhlova (Dept. of Acoust., Phys. Faculty, Moscow State Univ., Moscow 119899, Russia, vera@na.phys.msu.su), Peter J. Kaczkowski, and Lawrence A. Crum (Univ. of Washington, Seattle, WA 98105)

A promising therapeutic application of high-intensity focused ultrasound (HIFU) is hemostasis. In one possible hemostatic application, ultrasound induced heating will be used to stop bleeding during open surgery. To achieve substantial heat deposition on the surface of the bleeding organ, intense acoustic fields will be used, and therefore nonlinear effects on wave propagation in the coupling liquid between the transducer and the treated region must be taken into account. Moreover, nonlinearity can be used to increase the efficiency of heat deposition in shallow tissues by transforming wave energy to higher harmonics. Theoretical characterization of acoustic field is performed using a one-dimensional spherical wave model and the KZK equation. On-axis and off-axis waveforms, spatial distributions of harmonics, acoustic intensity, and heat deposition are calculated numerically in water and in tissue using a frequency-domain scheme. The influence of operating frequency, initial intensity, transducer aperture, amplitude shading, focal depth, and position of the water-tissue interface on acoustic propagation regimes and associated tissue heating is studied. For given absorption properties of the tissue and desirable spatial shape of the thermal lesion, an optimal set of transducer parameters and corresponding nonlinear effects are presented and discussed. [Work supported by DARPA, ONR, CRDF and RFBR.]
3aBB8. Effect of tone burst ultrasound in combination with PPAA (poly-propyl acrylic acid) on hemolysis of erythrocytes. Tyrone M. Porter, Pierre Mourad, Larry Crum (Appl. Phys. Lab., 1013 NE 40th St., Seattle, WA 98105), Niren Murthy, Alan Hoffman, and Pat Stayton (Univ. of Washington, Seattle, WA 98105)

In this study, high intensity focused ultrasound (HIFU) and PPAA (poly-propyl-acrylic acid) were combined at sublethal doses to achieve hemolysis of human erythrocytes. In a series of experiments, a suspension of erythrocytes (1A passive cavitation detection system) was utilized to monitor the acoustic emissions from the cell suspension during exposure to ultrasound. In the presence of PPAA, there is a significant increase in cavitation and hemolysis during ultrasound exposure at a $p \mathrm{H}$ of 6.1 , but not at a $p \mathrm{H}$ of 7.4. PPAA is a $p \mathrm{H}$-sensitive polymer, changing its conformation and its activity as the solution becomes more acidic, achieving its optimal active state at a $p \mathrm{H}$ of 6.1 . The acoustic data and the activity of PPAA suggest that the polymer, in its active state, provides nucleation sites to the cell suspension and that cell/bubble interaction is the primary mechanism for cell damage.

\section{1:00}

3aBB9. The effects of temperature on the characteristics of the focal region in high-intensity focused ultrasound. Francesco P. Curra, Pierre D. Mourad, and Lawrence A. Crum (Appl. Phys. Lab., Univ. of Washington, Seattle, WA 98105)

The application of high-intensity focused ultrasound uses the transformation of ultrasonic energy into thermal energy to raise the temperature of tissue rapidly and induce necrosis of tumor cells. Targeting and control of the heated region and the amount of energy delivered are important issues. These numerical simulations couple sound propagation with the bio-heat equation to investigate tissue heating over time. The temperature rise affects both sound speed and attenuation. These, in turn, influence the spatial boundaries and magnitude of the ultrasonic time average intensity which, in turn, drives the temperature. In this model, the dependence of the sound speed and tissue attenuation on temperature is based on experimental results obtained in pig liver [Bloch et al., Proc. 16th Intl. Cong. Acoust., Seattle, WA, 1065-1066 (1998)]. The results show a spatial shifting of the focal region toward the transducer accompanied by a reduction in the magnitude of the ultrasonic time average intensity depending upon the on-time of the transducer and the initial pressures at which it is driven. The temperature response follows the same behavior, with a spatial shift of the hottest spot and a decrease in its peak value, although thermal conduction tends to counteract the process. [Work supported by DARPA.]

\section{1:20}

3aBB10. High-intensity ultrasonic spectra in water and brain tissue. Kristian Jambrosic, Bojan Ivancevic (Faculty of Elec. Eng. and Computing, Unska 3, HR-10000 Zagreb, Croatia, kristian.jambrosic@fer.hr), and Igor Zoric (Faculty of Mining, Geology and Petroleum Eng., HR-10000 Zagreb, Croatia)

A common method of brain tumour surgery is using CUSA (cavitation ultrasonic surgical aspirator). While using CUSA, there is a strong focusing of the ultrasonic field within the cranium. This can cause damage to healthy brain tissue. Therefore, the ultrasonic field intensity at the critical spots must be measured. These measurements cannot be made in vivo, thus raising the need for a substitute model. In this paper, frequency spectra of the CUSA probe was measured at its working frequency of $25 \mathrm{kHz}$ in an oval container (simulating the cranium). The measurement was made at few field intensities, using the B\&K hydrophone in the container, and the $\mathrm{B} \& \mathrm{~K}$ microphone outside it. The medium temperature has been controlled. Calf brain tissue, physiologically similar to human, was used as a substitute model. The same measurement was repeated in water to examine the spectrum difference in high- and low-attenuation media. During the measurement, the occurrence of the cavitation was observed by spectra changes. 


\title{
Session 3aEA
}

\section{Engineering Acoustics and Underwater Acoustics: Recent Advances in Underwater Transducers I}

\author{
Dehua Huang, Cochair \\ Arichell Technologies, Inc., West Newton, Massachusetts 02165, USA \\ William J. Marshall, Cochair \\ BBN Systems and Technologies, Union Station, New London, Connecticut 06320, USA \\ Lars Kofoed, Cochair \\ Brüel \& Kjær, 2850 Nærum, Denmark
}

Chair's Introduction-7:55

Invited Papers

\begin{abstract}
8:00
3aEA1. A new deep-submergence broadband multiport underwater sound transducer. John L. Butler and Alexander L. Butler (Image Acoustics, Inc., 97 Elm St., Cohasset, MA 02025)

The back radiation from a transducer is normally isolated from the front radiation in order to avoid cancellation due to the out-of-phase nature of the back radiation. However, this isolation is difficult to achieve if the transducer is broadband and operated under significant ambient pressure encountered in deep submergence applications. The multiport transducer (U.S. Patent 5,184,332) offers a solution to this problem by using free-flooded resonance chambers on both the front and back surfaces of the transducer. It will be shown that with these chambers the acoustic pressure cancellation is avoided and the pressure now adds at frequencies between the two resonance's (which set the bandwidth of the system). A multiport design for a coaxial set of cylinders driven by the two surfaces of a piezoelectric ring transducer will be presented. Results from finite element and circuit analysis models will be presented and shown to agree with measured results on the model fabricated by Massa Products Corporation, Hingham, MA. [Work supported in part by an SBIR through NUWC, Newport, RI.]
\end{abstract}

8:20

3aEA2. 1-3 piezocomposite underwater transducers. Thomas R. Howarth (Naval Res. Lab., Washington, DC 20375-5350, howarth@nrl.navy.mil)

After over a decade of research (including application in medical ultrasonic products), 1-3 piezocomposite materials are now being utilized in a variety of U. S. Navy underwater transducer applications. This paper will survey a selection of current transducers that feature 1-3 piezocomposites as the active elements. The overview will chronologically present the transducers as they have evolved from the initial design of shaped aperture transceivers for acoustical environmental studies followed by a broadband projector for synthetic aperture sonars. Other transducers include cylindrical- and spherical-shaped constant beamwidth transducers (CBT), a parametric array, subbottom profilers, and acoustic calibration standards. An advanced design featuring an integrated accelerometer, acoustic pressure sensor, and actuation layer will be included. The 1-3 piezocomposite material attributes that are making these transducers useful for underwater applications will be discussed. The direction of future 1-3 piezocomposite-based underwater transducers will conclude the presentation. [This research was supported by the Office of Naval Research Code 333.]

\section{8:40}

3aEA3. Biased lead zirconate titanate as a high-power transduction material. James M. Powers (Newport Div., Naval Undersea Warfare Ctr., Newport, RI 02841), Mark B. Moffett (Georgia Tech Res. Inst., Atlanta, GA 30318), Michael D. Jevnager (Naval Undersea Warfare Ctr., Newport, RI 02841), and Stephen S. Gilardi (Interface Eng., Mystic, CT 06355)

High-power, underwater transducers using polarized piezoelectric ceramic material are usually limited in drive amplitude so that depolarization does not occur, but application of a dc bias field in the polarization direction allows the use of higher ac drive fields. To demonstrate the feasibility of a biased operation as a means of achieving higher power, a thin-walled, spherical-shell transducer was constructed of Channel 5800 and tested in NUWC's Acoustic Pressure Tank Facility. The transducer was successfully driven to $33 \mathrm{~V} / \mathrm{mil}(1.3 \mathrm{MV} / \mathrm{m}) \mathrm{rms}$ with an accompanying bias field of $31 \mathrm{~V} / \mathrm{mil}(1.2 \mathrm{MV} / \mathrm{m})$ and a hydrostatic pressure of $1400 \mathrm{psig}(9.65$ $\mathrm{MPa}$ ). The source level was $206 \mathrm{~dB} / / \mathrm{mPa}-\mathrm{m}$ at $61 \mathrm{kHz}$, corresponding to a 10-dB improvement over the usual Navy standard unbiased drive limit of $10 \mathrm{~V} / \mathrm{mil}(0.4 \mathrm{MV} / \mathrm{m}) \mathrm{rms}$. [Work supported by ONR.] 
3aEA4. Steered beams using a spatially phased array. W. Jack Hughes (Appl. Res. Lab., Penn State Univ., P.O. Box 30, State College, PA 16804,wjh2@psu.edu)

A spatially phased transducer array has been developed and tested that can form a steered beam without the need for electronically applying phase shifts to an array of elements. In its simplest form, the transducer consists of two channels from spatially shaped cosine and sine apertures, where the sine channel is shifted 90 degrees and then combined with the cosine channel. The transducer produces a beam steered to a desired angle at a specific design frequency in either receive or transmit operation. Several have been fabricated and tested using low-lateral mode transducer materials such as polyvinylidene flouride (PVDF) and 1-3 PZT composite, and electroplated electrodes to form the cosine and sine apertures. The appearance of the electrode has led to calling this a "doily" transducer array. Beams were formed that were steered as far as 54 degrees with typical sidelobe levels of $-20 \mathrm{~dB}$. The apertures were also geometrically shaped to supress sidelobes along the steering axis. [This work was supported by Kam Ng of ONR.]

9:20

3aEA5. Recent low-frequency sonar transducers and arrays. Didier Boucher (DCN Ingénierie centre Sud, sous-direction lutte sous la mer, BP 30, 83800 Toulon Naval, France)

Research in the field of high-power, low-frequency underwater transducers has been sustained by advances in submarine noise reduction and the promise of long-range detection at low frequencies. The earliest idea came up to use the already available passive towed array sonars as receive arrays in conjunction with additionally towed sound sources. The frequency of the active adjunct idea was of course given by the existing passive arrays. Very promising trials at sea, even in the shallow water environment, soon led to the consideration of full low-frequency active sonar systems (LFASS). Those have now been investigated for nearly two decades. With the emergence of new high-power transduction materials and the rapid evolution of fast computers and powerful modeling techniques, this research field has been fertile ground for transduction innovation. Specifically, high-power transducer developments include performance improvements to conventional piezoelectric ceramic projectors, progress in the exploitation of new active driver materials, and advances in transduction design techniques. While high power, low frequency, wide bandwidth, and high efficiency remain the primary performance objectives behind these developments; low weight, small size as well as medium to deep submergence are desirable physical attributes that require equal attention by the designer. This paper emphasizes the key characteristics of two sea-proven concepts for LFASS: Janus Helmholtz transducers and multihead arrays (international patents). The attention will be particularly focused on measured values of the sound sources. Finally, this paper addresses future trends in terms of sonar array designs for LFASS.

9:40

3aEA6. Recent developments of underwater acoustic transducers in China. Houqing Zhu (Inst. of Acoust., Chinese Acad. of Sci., Beijing 100080, PROC, zhu@tank.ioa.ac.cn)

Some of the recent developments of underwater acoustic transducers in China, such as the Rare-Earth giant magnetostrictive (Terfenol-D underwater acoustic transducers (including 1-kHz free-flooded modified IV flextensional transducer, the 890-Hz freeflooded Terfenol-D hexagonal transducer, and the $2.7-\mathrm{kHz}$ Tonpilz Terfenol-D transducer), the $500-\mathrm{Hz}$ flexible disk piezoelectric high-power transducer, the $450-\mathrm{kHz} 2-2$ piezoelectric composite underwater acoustic transmitting transducer, the 1-3 piezoelectric composite hydrophone, the 100-10 000-kHz polar glass ceramic hydrophone, the small-size low-frequency narrow beam hydrophone, the acoustic pressure receiver-vibration pick-up compound hydrophone, the large area PVDF hydrophone, etc., are presented in this paper.

10:00-10:20 Break

\section{Contributed Papers}

10:20

3aEA7. Development of a compact suspension system for a neutrally buoyant underwater acoustic intensity probe. James A. McConnell (Acoustech Corp., P.O. Box 139, State College, PA 16804)

Practical use of neutrally buoyant underwater acoustic intensity probes requires a suspension system to position them in some preferred orientation with respect to an acoustic field of interest. However, since these probes typically contain a moving coil geophone to facilitate particle velocity measurements, the design of such suspension systems is not trivial. On one hand, theory mandates that the dynamics of the geophone will not be adversely affected by the suspension as long as the mass-spring system created by the probe and the elastic members has a low-resonance frequency and high quality factor relative to that of the geophone. However, by meeting these two design criteria, wave effects (e.g., flexural resonances) in the elastic members can become a limiting factor in the performance of the device, particularly at high frequencies. This paper presents theoretical and experimental results of an effort to design a compact suspension system that embodies the aforementioned design considerations, but has relatively low susceptibilty to flexural resonances. [Work sup- ported by the Acoustics Technology Branch of the Naval Air Warfare Center-Aircraft Division, via the DoD Small Business Innovation Research Program.]

\section{0:40}

3aEA8. Comparative study: Flow-induced noise on underwater acoustic pressure, particle velocity, and intensity sensors. James A. McConnell (Penn State Univ., Grad. Prog. in Acoust., P.O. Box 30, State College, PA 16804 and Acoustech Corp., State College, PA 16804, univibe@sabine.acs.psu.edu) and Gerald C. Lauchle (Penn State Univ., State College, PA 16804)

A neutrally buoyant underwater acoustic $p-u$ intensity probe contains discrete sensors for direct measurement of acoustic pressure and particle velocity [J. A. McConnell and G. C. Lauchle, J. Acoust. Soc. Am. 103, 2755(A) (1998)]. Intensity is calculated from the cross spectrum between these two quantities. Thus a single probe can be categorized as a compound sensor capable of independently measuring acoustic pressure, particle velocity, and intensity. Using this probe, a comparative study concerning the measurement of all three quantities in the presence of mean flow and an independent sound source was performed. Bias errors, which 
result from the flow-induced noise generated by towing the sensor (e.g., a cylindrical body) in cross flow at low speeds through a channel of water, are presented. Other parameters of interest include analysis of experimental pressure-pressure and pressure-velocity correlation functions. The effect of body geometry (e.g., variation in aspect ratio) on the measured bias errors and correlation functions is also presented. [Work supported by the Office of Naval Research, the Naval Submarine League, and the Acoustics Technology Branch of the Naval Air Warfare Center-Aircraft Division, via the DoD Small Business Innovation Research Program.]

\section{1:00}

3aEA9. Bias error due to flow-induced noise on a velocity gradient underwater acoustic intensity sensor. Kevin J. Bastyr and Gerald C. Lauchle (Penn State Univ., Grad. Prog. in Acoust., P.O. Box 30, State College, PA 16804, zabour@sabine.acs.psu.edu)

A neutrally buoyant underwater acoustic $u-u$ intensity probe employs discrete sensors for the direct measurement of acoustic particle velocity at two locations. Active intensity is calculated from the cross spectrum between the two velocity sensors [K. J. Bastyr and G. C. Lauchle, J. Acoust. Soc. Am. 103, 2755(A) (1998)]. Under quiescent conditions the sensor has no bias error; however, in the presence of mean flow, this is no longer true. A derivation of the bias error that results from performing acoustic intensity measurements in the presence of mean flow is presented. Results show that the bias error due to the turbulent boundary layer generated by fluid flow over the cylindrical body that encapsulates the sensors is proportional to the cross spectrum of the turbulent velocities measured by the sensors. This theoretical derivation is quantified with preliminary experimental data obtained by towing the sensor at low speeds through an open channel of water. [Work supported by Office of Naval Research, MultiUniversity Research Initiative on Acoustic Transduction, Materials, and Devices.]

\section{1:20}

3aEA10. Development and testing of an ultrasonic PMN transducer. Jocelyne Coutte, Christian Granger, Bertrand Dubus, and Jean-Claude Debus (IEMN, Dept. ISEN, UMR CNRS 9929, 41 Bd. Vauban, 59046 Lille Cedex, France, jocelyne.coutte@isen.fr)

Lead magnesium niobate ceramics (PMN) are promising materials for realizing high-power transducers for macrosonics or underwater acoustics because of the large strains at moderate electric field and the low hysteresis. The use of PMN ceramics in transducers presents some difficulties: highly nonlinear properties, temperature and frequency dependence of dielectric permittivity, and dc bias field needed. This paper describes the design, realization, and testing of a $20-\mathrm{kHz}$ transducer. The Langevin-type transducer is made of four PMN-PT rings prestressed by a steel bolt between two-end-masses. The transducer is modeled using electrostrictive finite elements [J. C. Debus et al., J. Acoust. Soc. Am. 103, 3336-3343 (1998)]. It is optimized in terms of coupling coefficient and radiated acoustic power for in-air or in-water applications. The transducer is tested for several temperatures and various bias voltages and prestresses. Resonance and antiresonance frequencies, coupling coefficients, and vibrational amplitudes are measured and compared to numerical predictions and to experimental results on a reference piezoelectric transducer. [Work supported by a DRET/DCN grant.]
11:40

3aEA11. Thermal analysis of high drive transducer elements. Robert Montgomery and Stephen C. Butler (Naval Undersea Warfare Ctr., Newport, RI 02841, butlersc@code20nl.npt.nuwc.navy.mil)

The increasing demand for high-power, low-cost, compact transducer packages for underwater acoustic applications is leading to concerns of overheating in the active driving piezoelectric ceramic (lead zirconate titanate) elements and the associated passive materials (epoxies and polyurethanes). Pushing a design to its thermal limits can lead to reduced acoustic performance and reliability. There is not much guidance to be found in the literature that provides an analytical approach to cope with thermal issues of piezoelectric ceramics in the design phase. An analytic modeling effort is presented that addresses the thermal issues for compact high drive 31-mode free-flooded ring underwater sonar transducers. In this particular case, the thermal issues proved to be the limiting design concerns over the field and stress-limited cases. However, with modeling (analytical and finite element) and bench testing of components, the proper choice of materials and configuration parameters were determined and the performance goals were subsequently achieved. The approach addresses both the transient thermal response and the steady-state temperature profile that results from high-power, high duty cycle drive. These results may prove useful for other similar designs. [Work supported by ONR.]

\section{2:00}

3aEA12. The calibration of extended stationary low-frequency hydroacoustic antennae in the real conditions. Alexander $\mathrm{V}$. Agranovski, Byneev R. Osman, Sergei G. Cygankov, and Alexander V. Rosenberg (SPECVUZAVTOMATIKA Design Bureau, Lab. of Hydroacoustics R \& D, 51 Gazetni St., 344007 Rostov-on-Don, Russia, asni@ns.rnd.runnet.ru)

As known, the complex problems of a sea polygon calibration must include solutions of coordinate problems after an antennae systems placement was completed. At calibration, a necessity more frequently arises in a solution of other kinds of coordinate tasks: geometrical form determination for a receiving system; geometrical form determination for a receiving system and coordinates of the main receiving element in the selected immovable coordinate system (absolute fixing); and the self-coordinated determination of a mutual position for emitting points and receiving system elements. The first and second tasks assume that point coordinates are known (e.g., ones were determined by navigation methods), but at a third task they are not known. In the last case, a mutual location of hydrophones and emitting points ought be determined only by means of hydroacoustic measurements. At calibration, other kinds of acoustic signals are used: continuous signal (tonal or narrow-band) and pulsed signal (explosion, pneumatic gun, etc.). In this paper some algorithms for the decision of the above-mentioned tasks at the calibration of extended stationary lowfrequency hydroacoustic antennae by using different kinds of emitted signals are described. 


\title{
Session 3aED
}

\section{Education in Acoustics: European Demonstrations in Acoustics Education}

\author{
Lothar Gaul, Cochair \\ Institut für Mechanik, Universität Stuttgart, Pfaffenwaldring 9, 70550 Stuttgart, Germany \\ P. K. Raju, Cochair \\ Department of Mechanical Engineering, Auburn University, Ross 201, Auburn, Alabama 36849, USA \\ Sally G. Revoile, Cochair \\ (University of Maryland, Baltimore), Sea Island, Georgia 31561, USA \\ Chair's Introduction-8:35 \\ Invited Papers
}

\begin{abstract}
8:40
3aED1. Application of numerical techniques in teaching technical acoustics. Fulop Augusztinovicz (Tech. Univ. of Budapest, Dept. of Telecommunications, Sztoczek u. 2., H-1111 Budapest, Hungary, fulop@ hit.bme.hu)

Due to their highly extended visualizing capabilities, numerical methods are very apt at demonstrating acoustic effects and phenomena. Finite-element methods (FEM) are mainly used to describe the sound fields in closed spaces, and boundary element methods (BEM) are well suited to predict radiation from vibrating bodies. Coupled systems can also be analyzed by some hybrid approaches. The paper shows applications of these tools in undergraduate and postgraduate courses of technical acoustics. Starting from the well-known concentrated parameter approach, a more realistic description of basic acoustic elements and systems consisting of acoustic masses, acoustic compliances, Helmholtz resonators, waveguides, etc. can be presented. Based on these examples, the dynamic behavior of acoustic systems such as the existence of standing waves, eigenmodes, and resonance effects can be visualized and understood. Radiation problems can be solved for elementary acoustic sources, demonstrating the basic rules of fluid-structure interaction phenomena. The most advantageous feature of numerical-FEM/BEM-calculation and visualization techniques is its capabilty to handle a large amount of data. Problems can be approached in three dimensions, both in time and frequency domain. Apart from the application areas, the lecture addresses the necessary hardware and software tools to prepare these sorts of demonstration, too.
\end{abstract}

\section{9:00}

3aED2. A library of visualization and auralization examples to support understanding. Jens Blomqvist, Tor Kihlman, and Wolfgang Kropp (Dept. of Appl. Acoust., Chalmers Univ. of Technol., SE-41296 Gothenburg, Sweden, wk@ta.chalmers.se)

In a project financed by the Swedish Council for renewal education a library has been produced containing visualization and auralization examples concerning physical phenomena in acoustics. The main goal of the library is to support the development of a thorough understanding of basic acoustic phenomena. The work was supported by studies in the form of interviews and questionnaires to understand-What are the genuine problems students have in acoustics, mathematics, and physics? How do the students' and teachers' view of acoustics, physics, and mathematics differ? What are suitable measures to achieve a common basis between teacher and student for a successful communication? During the project it became obvious such a library has to be developed together with students from our courses. The library has to be adapted to the students needs and to their mental picture of physics, mathematics, and acoustics. In order to achieve this, a group of students was asked to take part in the project. They directed both content and layout of the library from their own perspective. The results of this work will be presented together with typical examples from the library.

\section{9:20}

3aED3. Web-accessible resources for teaching phonetics. Anders Eriksson (Dept. of Phonet., Umeå Univ., S-901 87 Umeå, Sweden, anderse@ling.umu.se)

The paper covers two aspects of the usability of the Internet resources in teaching phonetics. First of all, the Internet may be used to disseminate information and teaching materials. Search facilities provided by normal, search engines are too coarse, however, to be really useful if one wants to find suitable teaching resources (course notes, tutorials etc.). One way to address this problem is to use a structured inventory of hyper-links to relevant materials. A working group within the Socrates/Erasmus thematic network on Speech Communication has set up a searchable inventory of web accessible materials, relevant to teaching phonetics. The principles underlying the structuring of the database will be described and illustrated by examples from the database. A second possible use has to do with Open and Distance Learning (ODL). Phonetics, however, requires both sounds and images to be realistic, and for many areas the possibility to perform experiments is essential. With the advent of internet-based tools like audio and video players and programming languages like Java new possibilities have opened up for realistic, high-quality phonetics resources. The presentation will demonstrate a perception test, which may be run inside a normal web browser and which is accessible over the Internet. 
3aED4. Model of the human speech production based on vocoder techniques. Klaus Fellbaum (Brandenburg Tech. Univ., Cottbus, Germany) and Joerg Richter (Tech. Univ. of Berlin, Berlin, Germany, fellbaum@kt.tu-cottbus.de)

A tutorial is presented with which the student first gets basic knowledge about the human speech production. It is explained that speech is generated by an air flow coming from the lungs which is, in the case of voiced sounds, periodically interrupted by the vocal cords and then modulated with the resonance rooms of the mouth and nasal tract. For voiceless sounds (like "f," "sch") the vocal cords are open and the air flow excites the mouth and nasal tract with a noise-like pressure signal. It is therefore adequate to distinguish two components in the speech production: the excitation and the articulation component. In our tutorial, the two components are realized (simulated) by a technical system, known as linear predictive vocoder (LPC vocoder). Its principle is explained by a short text and some graphics and the analogy to the human speech organs is clearly shown. The main emphasis is put on the control parameters of the system - in the main the pitch frequency and the vocal tract parameters (prediction coefficients)—and the effect when.

\section{0:00-10:20 Break}

\section{$10: 20$}

3aED5. Teaching boundary element methods in acoustics. Lothar Gaul, Marcus Wagner, and Wolfgang Wenzel (Univ. Stuttgart, Inst. A fuer Mechanik, Pfaffenwaldring 9, 70550 Stuttgart, Germany, L.Gaul@ mecha.uni-stuttgart.de)

The boundary element method (BEM) provides a powerful tool for the numerical solution of sound fields, because (1) it maps the domain equation on the boundary and thus reduces the problem dimension by one, (2) it provides a nonreflecting boundary on an arbitrary radiating surface, and (3) pressure, velocity, and intensity can be calculated at every field point from the boundary data. The author's concept of teaching BEM in a graduate engineering course is to provide a link with subjects taught in undergraduate engineering mechanics such as rod vibrations, reciprocity principle, influence functions, and finite elements (FE). These subjects are generalized to the field equations of acoustics and elastodynamics. The associated integral equations are derived from the dynamic reciprocity principle. Their kernels are fundamental solutions which turn out to be influence functions of a full space. Boundary formulations of rods and beams recall well-known FE matrices. The fluid-structure interaction of interior and exterior acoustics is formulated by adopting the unsymmetric allocation approach of BEM and a symmetric hybrid BEM which is based on a multifield varational principle. Waves and vibrations in a flexible pipe and the sound radiation from a vibrating tire are treated as examples.

\section{0:40}

3aED6. An adaptive, active noise reduction system in closed space. Andrzej Golas and Wojciech Ciesielka (Dept. of Mech. and Vibroacoustics, Univ. of Mining and Metallurgy, Poland, ghgolas@cyf-kr.edu.pl)

In the demonstration an adaptive system of noise reduction is presented. Both the acoustic signals phase compensation and the adaptive digital filtering theory are employed. An incident acoustic wave is identified and a compensation signal is generated using a finite impulse response filter which coefficients are calculated in real time employing an LMS algorithm. The algorithm is implemented on a dSpace card with a signal processor (TMS320C31 Texas Instrument). In the receiving point (artificial head, real listener, receiving transducer) the disturbing signal (white noise filtered in range $50-250 \mathrm{~Hz}$ ) is reduced by an active adaptive system. The damping coefficient that is obtained in the system varies from 10 to $20 \mathrm{~dB}$. The experiment can be conducted and observed in any interior.

\section{1:00}

3aED7. The use of computers in acoustical engineering: A computer-aided electroacoustics course. Juan J. Gomez Alfageme, Manuel Recuero, and Jose L. Sanchez (Dept. of Audiovisual and Commun. Eng., Polytechnic Univ. of Madrid, Ctr. Valencia Km. 7, 28031 Madrid, Spain, alfageme@diac.upm.es)

The teaching of electroacoustics in the audiovisual engineering curriculum has sustained important changes during the last 4 years. The increasing use of computers in the classroom has allowed a new approach to this subject, which needs vast knowledge in physiscs, mathematics, and signal analysis. In the last years our department has developed a computer-aided course in electroacoustics. This course is made in the Windows operating system and programmed in Matlab, for its extended use in engineering departments all over the world. At the moment, the implemented modules are electroacoustic transducer analysis, direct radiation loudspeaker systems, array directivity, sound intensity, electrodynamic loudspeaker distortion, and speech index transmission calculus. All the modules have the same structure using the Matlab object-oriented user interface facilities and use the Matlab resources for graphical representation. Each module makes transducer behavior simulation based on equivalent circuits. The student does not need to have a great deal of knowledge in programming to work with the modules. The structure of different modules will be explained and the results with students in our curriculum will be provided.

\section{1:20}

3aED8. Acoustics education at the Stuttgart University. Gerhard Hübner (Universität Stuttgart, ITSM, Pfaffenwaldring 6, D-70550 Stuttgart, Germany, wittstock@itsm.uni-stuttgart.de)

The Stuttgart University is relatively young compared to several other German universities. Nevertheless or fortunately for this reason the developments in Stuttgart's acoustical teaching went off with some remarkable distinctions in its relevant scientific history and present situation. (i) A new teaching focused on noise generation and radiation caused by practical sound sources was started successfully by K. Gösele already in the late 1940s. (ii) Supported and initiated by the requirements of the important industrial area around Stuttgart, an increasing interest of more and more Engineering Departments to include noise and noise control in lectures and 
investigations took its origin much earlier than in other places which, in 1993, concluded in the establishment of the "Work Group of Technical Acoustics and Noise Control at the Stuttgart University" (ATALUS) where 13 institutes from 6 different faculties organized their exchange of experiences on a broad basis. Furthermore, in 1994 several acoustical lectures are included in the new curriculum "environmental engineering and protection." (iii) Several relevant basic lectures are straightened on the general theory, e.g., focused on numerical methods (BEM, the link of DFEM with the physical phenomenon of sound radiation), propagation and sound power of structure-borne sound in general shaped bars and shells using second-order differential equations, and sound intensity measurement techniques.

\title{
11:40
}

3aED9. Standard and nonstandard Galerkin FEM for numerical acoustics. Frank Ihlenburg (Germanischer Lloyd, Vorsetzen 32, D-20459 Hamburg, Germany, ihl@hamburg.germanlloyd.de)

Over the last decade, several modern nonstandard modifications of the standard Galerkin finite-element method have been developed for, or applied to, the numerical solution of the Helmholtz equation. Most of these concepts, like Galerkin least-squares or bubble methods, have been first developed and demonstrated in one dimension, where also a rigorous analysis of the so-called preasymptotic error behavior of the standard Galerkin FEM is available. In this talk, a short overview of these topics will be given and the extension of the methods/results to higher dimensions will be discussed, where practical problems are formulated.

12:00

3aED10. Courses on acoustics: What is needed? Otto von Estorff (Ocean Eng. II, TU Hamburg-Harburg, D-21071 Hamburg, Germany, estorff@tu-harburg.de)

The constant reduction of allowable noise levels and consumer demand for a better acoustic performance of technical systems put a tremendous pressure on manufacturers. As a consequence, engineers who know about acoustics, and the measures to improve it, are currently looking at numerous job openings. In fact, the industry is seeking intensively in this field. The present paper is related to the contents of courses on acoustics at the university as well as in industry. Its focus is to discuss the question "What is needed?' in order to meet the requirements of future acoustic challenges and, of course, the expectations of the student's future employer. Based on the author's experience and a little questionnaire, a possible course content is setup and discussed.

\section{Session 3aMU}

\section{Musical Acoustics: Musical Instruments and Structural Acoustics I: Experimental Studies, Theoretical Models and Numerical Analysis}

\author{
Antoine J. Chaigne, Cochair \\ ENST, Dept. TSI, CNRS URA 820, 46 Rue Barrault, 75634 Paris, Cedex 13, France \\ Uwe J. Hansen, Cochair \\ Department of Physics, Indiana State University, Terre Haute, Indiana 47809, USA
}

Chair's Introduction-7:55

Invited Papers

8:00

3aMU1. Experimental and theoretical studies of the modes of stringed instruments and their relevance for quality control of instrument manufacture. Bernard E. Richardson (Dept. of Phys. and Astron., Univ. of Wales, Cardiff, P.O. Box 913, Cardiff CF2 3YB, UK, RichardsonBE@Cardiff.ac.uk)

This paper reviews various methods for measuring and predicting the modes of vibration of the wooden structures found in instruments of the violin and guitar family. These methods include holographic and speckle interferometry, modal analysis by impact hammer excitation, and the finite element method. Theoretical modeling of stringed instruments has now advanced to the stage where the models can be "played," allowing listening tests to be used to link construction of the instrument with the perceived sound quality in a formal way. Early indications of this work suggest that mode frequencies play a much smaller role in determining the sound quality of an instrument than has previously been assumed, and that greater attention should be paid to the "ease" with which modes can be driven by the strings and to the "source strengths" of the modes. This paper will put the case for measuring additional modal parameters, such as effective mass and effective stiffness, and discuss their relevance to quality control by the maker during manufacture of an instrument. 
3aMU2. Dynamical behavior evolution of the guitar harmonic plate in the successive construction phases. María J. Elejabarrieta (Dept. Mecánica, Escuela Politécnica Superior de Mondragón Unibertsitatea, Spain), Amaya Ezcurra (Univ. Pública de Navarra, Spain), Carlos Santamaría (Univ. del Pais Vasco, Spain), and José Bretos (Univ. Pública de Navarra, Spain)

The fundamental piece for the quality of the sound of a guitar is its harmonic plate. From the initial phase (gross plate without hole) until the last construction phase (the addition of the structures system), the craftsman has established a series of stages where the acoustical behavior is modified until the definitive guitar is created. This is the reason to establish the effect of each modification in the vibratory response of the harmonic plate. With this purpose an experimental modal analysis and a finite element modeling of all the construction phases in the low-frequency range have been carried out, obtaining information about vibration patterns, eigenfrequencies, quality factors, and spectra. In the three first construction phases, the plate goes from a uniform thickness distribution and no hole to a mouth and variable thicknesses. These changes do not modify the vibration patterns, but they lead to a gradual decrease of the eigenfrequencies and an increase of the vibration amplitude. The vibration patterns are modified when the structures are added. Then, the rigidity of the structure is increased and the resonances present a smaller damping. Likewise, the frequency response function decreases remarkably and the resonances tend to be more uniformly distributed in frequency.

\section{8:40}

3aMU3. Numerical simulation of timpani. Patrick Joly, Leila Rhaouti (INRIA, Domaine de Voluceau-Rocquencourt, BP 105, 78153 Le Chesnay Cedex, France), and Antoine Chaigne (ENST, 75634 Paris Cedex 13, France)

The goal of this work is to achieve sound synthesis via time-domain numerical simulations, with particular interest in the case of timpani. The approach combines the use of a mathematical model, which is supposed to represent accurately the physics of the phenomena, and of modern techniques for the approximation of partial differential equations. The model appears as a system of wavelike equations coupled by fluid-solid interaction conditions. It includes various damping mechanisms as well as a nonlinear model for the mallet-membrane interaction. The numerical approach aims at realizing the best compromise among accuracy, stability, and efficiency. Its main characteristics are the use of finite elements for the space discretization, of a fictitious domain method for a flexible treatment of the geometry of the instrument, and of explicit centered finite differences for the time discretization. Higher-order absorbing boundary conditions are used for simulating the radiation of sound in free space. In the presentation, the main properties of the numerical methods will be emphasized and various numerical results will be compared to experiments.

\section{9:00}

3aMU4. Comparison between modal analysis and finite-element modeling of a marimba bar. Antoine Chaigne (ENST, Dept. TSI, CNRS URA 820, 46 Rue Barrault, 75634 Paris Cedex 13, France) and Ingolf Bork (Physikalisch-Technische Bundesanstalt, Braunschweig, Germany)

The sound spectrum of low-tuned marimba bars contains many components that cannot be explained by a simple one-dimensional model of flexural vibrations. A modal analysis has thus been performed on a bass marimba bar $\mathrm{C}_{3}$ with a fundamental frequency of $130 \mathrm{~Hz}$ [outer dimensions: $46 \times 6 \times 2.35 \mathrm{~cm}(x, y, z)$, material: rosewood], taking into account two spatial components of vibration. The 20 lowest modes were extracted from this analysis (modal frequencies and mode shapes). A 3-D finite-element analysis of this bar, based on an orthotropic model for the material, has been conducted in parallel. Modal analysis and finite-element modeling yield very similar mode shapes and modal frequencies for the first 12 modes, between 130 and $4000 \mathrm{~Hz}$, which confirms the experimental results and validates the theoretical approach. The discrepancies between measured and calculated frequencies are less than $4 \%$ in this frequency range. For higher frequencies, between 4000 and $8000 \mathrm{~Hz}$, the main flexural modes along $0 x$, such as the $(7,0)$ and the $(8,0)$ modes, are still correctly predicted. In this range, the three components of the bar motion become significant and the finite-element modeling shows a number of modes which were not detected in the modal analysis.

\section{Contributed Papers}

9:20

3aMU5. Investigating the effect of different strutting arrangements on the modes of a guitar soundboard. Mark Lewney and Bernard E. Richardson (Dept. of Phys. and Astron., Univ. of Wales, Cardiff, P.O. Box 913, Cardiff CF2 3YB, Wales, m.lewney@astro.cf.ac.uk)

A spruce soundboard was fitted with a bridge and attached to an experimental rig which provided a fixed edge and uniform backing cavity volume. The rig allowed the same soundboard to be fitted with various standard strutting arrangements. The mode shapes and frequencies were investigated using holographic interferometry. Of particular interest were the small changes in mode shapes induced by the different strutting arrangements. Input admittance measurements were made to determine the effective mass of each mode as "seen" by the strings. Previous psychoacoustical work [H. A. K. Wright, Proc. Inst. Acoust. 19(5), 149-154 (1997)] has suggested that effective mass is an important parameter in determining a mode's contribution to the overall sound output, and can be significantly affected by small changes in the mode shape. A new method of determining the effective mass is also presented. [Work supported by EPSRC.]
9:40

3aMU6. Air cavity modes in sound boxes recorded by TV holography. Anna Runnemalm and Nils-Erik Molin (Div. of Exp. Mech., Lule Univ. of Technol., SE-971 87 Lule, Sweden, Anna.Runnemalm@mt.luth.se)

Amplitude and phase distribution of air cavity modes in a rectangular box, in a guitar, and in a violin are measured using TV holography. The box and the top and back plates of the guitar and the violin are made transparent using 5-mm-thick and flat PMMA plates. Standing waves in the enclosed cavities are generated by a loudspeaker. Object laser light is sent through the cavities to a rigid white-painted steel block and reflected back again through the cavities to the optical unit of the TV holography interferometer. Laser light traveling through a sound pressure field will experience a phase delay, since an increased air pressure is accompanied by a higher air density as well as a higher index of refraction which in turn gives a slower speed of the laser light, which generates a phase delay compared to undisturbed air. This often small phase change is made visible by the highly sensitive phase-modulated TV holography technique. The optical system will be described and air cavity modes in a simple rectangular box, in a guitar, and in a violin will illustrate the presentation. 


\section{Invited Papers}

\section{0:20}

3aMU7. On the function of the violin bridge. Erik V. Jansson (Dept. of Speech, Music and Hearing, Royal Inst. of Technol. (KTH), SE-100 44 Stockholm, Sweden, erik@speech.kth.se) and Benedykt K. Niewczyk (Violin Workshop, ul Wozna 6, 61-776 Poznan, Poland)

In measured frequency responses of violins of soloist quality a broad maximum is found at about $2.5 \mathrm{kHz}$ which has been called the bridge hill as the violin bridge on rigid support has its major in-plane resonant frequency nearby [E. V. Jansson, Acust./acta acust. 83, 337-341 (1997)]. Experiments show, however, that the hill rather lies in combined properties of the bridge and of the top plate. It can be influenced both by the properties of the top plate between the $f$-holes but also by the bridge. The maximum is efficiently excited in the normal main direction of bowing. The force along the bow is transformed into a force couple at the bridge feet which excites the bridge and body. The broad maximum involves resonant motion which is not confined to the bridge alone. [Work supported by KTH, Swedish Natural Science Research Council, Swedish Institute, and Wenner-Gren Center Foundations for Scientific Research.]

10:40

3aMU8. Normal modes of vibration in a violin. Uwe J. Hansen (Dept. of Phys., Indiana State Univ., Terre Haute, IN 47809) and Thomas D. Rossing (Northern Illinois Univ., DeKalb, IL 60115)

The normal modes of vibration of a violin constructed by Carleen Hutchins have been studied using both sinusoidal and impact excitation while the instrument is supported in several different ways. The results from these experiments are compared to those of several previous investigators on the same instrument [see references in Roberts and Rossing, Catgut Acoust. Soc. J. 3(5), 3-9 (1998)]. The observed operating deflection shapes, from which normal modes are determined, are found to vary with means of support and excitation, but by comparing the various data the normal modes can be determined with reasonable certainty.

11:00

3aMU9. Deconstructing the violin-The road from B to A. George Bissinger (Phys. Dept., East Carolina Univ., Greenville, NC 27858, bissingerg@mail.ecu.edu)

A recently initiated project to measure the elastic and damping material properties, normal mode frequency and shape vibrational properties, and the angular distribution and efficiency of radiation from assembled violins is described. Combining accurate geometries and densities, experimental modal analysis with a scanning laser vibrometer, measurements of bridge-corpus transfer mobilities, and far-field radiation over a sphere, one potential application is to extract the relevant material and vibrational properties for all substructures. Another goal is to generate a finite-element model "particularized" to each violin to calculate normal mode radiation from the violin and simulate its sound. Other aspects and applications of the model will also be briefly discussed. [Research supported by the National Science Foundation under Grant No. DMR-9802656.]

11:20

3aMU10. Finite-element analysis of vibrating trombone bell. P. L. Hoekje (Dept. of Phys. and Astron., Baldwin-Wallace College, 275 Eastland Rd., Berea, OH 44017) and Andrew Morrison (Univ. of Northern Iowa, Cedar Falls, IA 50614-0150)

Finite-element analysis (FEA) was used to predict the vibration patterns of an isolated trombone bell. These vibrations are implicated in subtle contributions to the sound field, over and above the primary contribution from the vibrating air column. Holographic interferometry of vibration patterns of an actual bell reveal that most modes exhibit rotational symmetry with vibrating patches of equal size distributed around circumferential rings. Such modes at low frequencies will radiate poorly. However, some patterns observed by holography do not display such symmetry and will have enhanced sound radiation as a result. FEA calculations using a mesh of points with rotational symmetry seem to give only symmetric modes, but lead to elements of unequal sizes and aspect ratios. On the other hand, using the package's AutoMesh feature to redraw the mesh into equal-sized elements for more efficient computation introduces inherent asymmetries into the model, and also breaks the symmetry of the solutions. It is concluded that the observed asymmetries in the holographic data are due to asymmetries in the bell itself, leading to enhanced radiation of sound. [Work supported by the Carver Foundation.]

\section{Contributed Papers}

11:40

3aMU11. Simulation and measurement of wall vibrations of a flue organ pipe. Malte Kob (Inst. of Tech. Acoust., Tech. Univ. Aachen, Templergraben 55, D-52056 Aachen, Germany) and Matthias Scholz (Ackerwand 21, D-99423 Weimar, Germany)

The question of whether the vibrations of a metal flue organ pipe contribute to the perceived sound or not is still not completely answered. One result of the research done within the GOArt (Göteborg Organ Art Center) project is that, due to the casting process and the need to save material, the baroque flue organ pipe has considerably thinner walls than a modern pipe. According to an organ builder, the pipe under test is likely to vibrate. To demonstrate the influence of the wall vibrations on the perceived sound, recordings of a blown pipe with and without damping of the wall are carried out. An FE model is created and the radiation for the case of coupled air and structural modes are calculated to enable parametric studies of the influence of the wall properties. The simulations are compared to FRF measurements using MLS excitation of a shaker, laser vibrometer velocity measurements, and subsequent modal analysis. The results indicate that some structural modes significantly change the spectral 
components in the transient spectra of the blown pipe, whereas in the stationary spectrum, the effect is small. [This work was performed at the Department of Applied Acoustics, Chalmers University of Technology, Göteborg, Sweden.]

\section{2:00}

3aMU12. An investigation of vibrational and acoustical properties of the violin using MLS and optical holography. Lars H. Morset (Appl. Optics Group, Inst. of Phys., Norwegian Univ. of Sci. and Technol. (NTNU), 7034 Trondheim, Norway, Lars.Morset@phys.ntnu.no)

In this investigation a multi-channel MLS (maximum length sequence) measurement system is used for vibrational and acoustical measurements on the violin. MLS measurements are useful since both phase and magnitude can be measured with great accuracy. In these measurements, both the phase and magnitude influence of the transducers are corrected. Using this tool, the input admittance, the velocity at the feet of the bridge, and the sound pressure at the far field of the violin are measured simultaneously. These parameters give important information about the vibrational and acoustical properties of the violin. A more extensive investigation is also performed. The sound level pressure and phase are measured in several equally spaced positions in the far field of the violin situated in an anechoic chamber. Radiation patterns are found for each mode and the radiated power is calculated. Optical holography is then used to measure the vibrational pattern for the most important modes of the violin body. These acoustical and vibrational measurements are correlated to determine which modes are most important to the radiated sound power.

WEDNESDAY MORNING, 17 MARCH 1999

ROOM H2032, 7:55 TO 9:20 A.M.

\title{
Session 3aNSa
}

\author{
Noise: Noise at the Workplace II \\ Gustav A. Sehrndt, Cochair \\ Consultant, Kapuzinerstrasse 8, D-50 737, Cologne, Germany \\ Alice H. Suter, Cochair \\ Alice Suter \& Associates, 575 Dogwood Way, Ashland, Oregon 97520, USA
}

Chair's Introduction-7:55

\section{Contributed Papers}

\section{8:00}

3aNSa1. Characteristics of the noise found in day-care centers. Picard Michel and Boudreau Chantal (Ecole d'orthophonie et d'audiologie, Universite de Montreal, Montreal, QC H3C 3J7, Canada, picardmi@magellan.umontreal.ca)

Today's day-care centers are apparently extremely noisy environments. Given the paucity of data on this particular issue, noise measurements were collected for 25 sites with groups of 1.5- to 5-year-old children $(N=4-16)$. Noise levels associated with three types of children's activities were studied, namely: unstructured activities of the children; structured ones (e.g., story telling); and lunch time. In addition, measurements were collected for the unoccupied case. A-weighted levels (10-90 percentiles, average levels, $L_{\mathrm{Aeq}}$ ), and third octave levels will be presented for each measurement condition. Statistical analysis to model the noise in all conditions will also be reported. Of special concern, $L_{\text {Aeq }}(10 \mathrm{~min})$ indicated frequent readings in excess of $85 \mathrm{~dB}$ for all measurement conditions except the unoccupied case. This suggests that children may be at risk of noise-induced hearing loss dependent on the length of stay in the day-care centers. They would only be able to initiate or sustain any meaningful verbal communication activity by using extreme vocal effort to speak loud enough to raise their voice above the din. Obviously, this is quite an alarming situation.

8:20

3aNSa2. Occupational noise in Spanish textile industries. Amando Garcia, Jose V. Garrigues (Dept. de Fisica Aplicada, Univ. of Valencia, Burjassot, Valencia, Spain, 46100, amando.garcia@uv.es), and Ana M. Garcia (Univ. of Valencia, Valencia, Spain, 46022)

This study has been carried out in 20 small-, medium-, and large-sized textile industries located in the provinces of Valencia and Alicante (Spain). A series of noise-level measurements has been carried out in order to evaluate the daily noise exposure levels $\left(L_{\mathrm{ep}, \mathrm{d}}\right)$ for a wide variety of workplaces. About $33 \%$ of the workers present during the measurements were exposed to daily mean A-weighted noise levels $L_{\text {ep,d }}$ higher than $85 \mathrm{~dB}$. It has been noticed that personal protection against noise was used only by a minority of these workers. Available data from routine medical examinations have been also analyzed. The observed hearing loss is related to noise exposure level in the corresponding workplaces. In particular, about $48 \%$ of the workers exposed to $L_{\text {ep,d }}$ levels higher than 85 $\mathrm{dB}$ showed acoustic trauma (hearing losses higher than $40 \mathrm{~dB}$ for the 4000-Hz frequency in either ear). 
3aNSa3. What is and should be in the area of noise-protection: Make progress a catalog on noise reduction. Karl Ross (DGUHT, Henlestr. 7, D-97074 Würzburg, Germany)

Noise has a harmful effect on health. Also, More than 10000 deaths every year in the ecological domain, for example, are said to be caused indirectly in this way in Germany. In the area of the industrial safety, the following applies: the regulation governing accident prevention under the title "Noise" has been in existence since 1975. Annually, more than four million persons are at noisy workplaces and two million hearing screening tests are being carried out. Yet, noise-related illnesses still occur (1996: 11724 reported illnesses/1252 pension-cases receiving compensation for the first time). The costs of these were over 500 million German Marks altogether. However, company practicians are often not able to plan/carry out noise reduction without intensive support. Therefore, there had been checked more than 200000 pages for the keynotes "noise reduction" or something similar. A new catalog on noise reduction is introduced at this point. It contains more than 400 examples. In it are listed in alphabetical order: the noise-problem with its $\mathrm{dB}(\mathrm{A})$ values before and after the decrease, the approximate cost of material, a short description of noise reduction, and a guide to the literature pertaining to special cases. Providing this time as a prototype free of charge. The costs, the protected area, or volume are partly estimated.

\section{9:00}

3aNSa4. Ladle crane noise. Jerzy W. Wiciak, Marek Iwaniec, and Ryszard J. Panuszka (Structural Acoust. and Intelligent Mater. Group, Tech. Univ. of Mining and Metallurgy, 30-059 Cracow, al. Mickiewicza 30, Poland, ghpanusz@kinga.cyf-kr.edu.pl)

The present paper considers the vibration analysis of the ladle crane (with hoisting capacity $Q=160$ tons) and noise caused by working units of the crane. The vibroacoustical investigations and theoretical analysis have been applied to the main bridge with box-trapezoid cross section. In the article there is shown comparison of the vibration acceleration and dynamic stress that have been measured in real structure with calculations obtained by means of the finite element method. The noise caused by working units of the crane have been presented for three operating conditions: when the tilting ladle was descending and was hoisting, and pass by load running of the teeming crane. [Work supported by TUMM DS 11.130.557, Cracow, Poland.]

\title{
Contributed Posters
}

\begin{abstract}
These posters will be on display in the Poster Gallery from Monday to Wednesday, 15-17 March. Authors will be at their posters
\end{abstract} from 10:00 a.m. to 12:00 noon on Wednesday, 17 March.

\begin{abstract}
3aNSa5. Nonoccupational noise and hearing capability of children and young adults. Detlef Schulz, Karin Künzel, and Lars Hentschel (Hochschule für Technik und Wirtschaft Mittweida, Fachbereich MPI, Postfach 1451, D-09644 Mittweida, Germany)
\end{abstract}

There has been considerable literature over the past decade ascertaining a worsening of the hearing capability of young persons. It is supposed that this may be caused by some peculiarities of the modern way of living, that is, man-made noise (e.g., noisy hobbies). To get insight in some components of the real noise exposure of young people dosimetric measurements were performed in schools as well as in discotheques and as spectators of sports events (football matches and some indoor sports like basketball). For discotheques equivalent sound pressure levels of 95-105 $\mathrm{dB}(\mathrm{A})$ are typical; for indoor sports they are in many cases on the same order of magnitude. Persons frequently attending such events may get an exposure which is comparable with occupational noise exposure above the daily average of $85 \mathrm{~dB}$. Furthermore, audiometric testings of children and young adults at the age of 14-25 years were performed. The highfrequency range $(8-16 \mathrm{kHz})$ was taken into special consideration, since it is assumed to be important for the early detection of noise-induced hearing threshold shifts. Hearing thresholds together with the results of questionnaires are presented showing an increasing threshold shift with age already at young people, especially in the frequency region above $10 \mathrm{kHz}$.

3aNSa6. Noise levels in dental practice. Hrvoje Domitrovic (Faculty of Elec. Eng. and Computing, Dept. of Electroacoustics, Unska 3, HR-10000 Zagreb, Croatia, hrvoje.domitrovic@fer.hr), Ivana Bagic (School of Dental Medicine, Gunduliceva 5, HR-10000 Zagreb, Croatia), and Bojan Ivancevic (Faculty of Elec. Eng. and Computing, HR-10000 Zagreb, Croatia)

Noise can cause unwanted masking of sounds, interference with speech, even pain and injury, and temporary or permanent loss of hearing. Concerning exposure of noise levels at the workplace, industrial plants and huge machines are usually considered. However, there are "clean" jobs where people are exposed to significant noise. One example, which will be treated in this paper, is dental practice. In this work it is intended to show the results of SPL measurements that were done at the Dept. of Pedodontics in the School of Dental Medicine Zagreb. One- and twochannel measurements were made using a sound level meter and an artificial head (head-and-torso simulator). Noise levels generated by various dental devices, their spectra, and differences between noise levels in left and right ear (in the dentist's usual working position) were shown. The sound power and subjective sound levels regarding sound source frequency and intensity were determined upon results of these measurements. Special attention was paid to ultrasound and infrasound components, as well as vibrations. Since two Faculty practices with a dozen of dental sets in each were at our disposal, influence of aging and wearing, particularly in mechanical devices, was also considered.

3aNSa7. The role of measurement standards in acoustics. Richard G. Barham (Natl. Physical Lab., Teddington, Middlesex TW11 0LW, UK)

Recent times have seen a marked increase in public awareness of noise as both a cause of nuisance and a hazard to hearing. Consequently, demand for acoustical measurements has grown and is now an important issue across an ever broadening range of industrial sectors. If these measurements are to have any technical validity, it is essential that they be founded on some physical reference quantity or primary standard. In practice, this is achieved by means of a defined measurement infrastructure. In the UK, the National Measurement System serves this purpose and it is the role of the National Physical Laboratory (NPL) to establish the primary measurement standards to underpin the system. While different applications deal with a range of acoustical quantities, they all derive in some way from sound pressure. The primary standard for sound pressure is realized through the calibration of laboratory standard microphones, using the reciprocity method. NPL has for many years been at the forefront of research in this field and most recently has introduced a fully revised reciprocity calibration facility. The paper describes the facility and highlights the latest technical developments. It also discusses the value of third-part accreditation of such facilities. 


\title{
Session 3aNSb
}

\section{Noise: Hearing Protector Measurements, Effects, Design and Performance}

\author{
Elliott H. Berger, Cochair \\ E·A R Hearing Protection Products, 7911 Zionsville Road, Indianapolis, Indiana 46268-1657, USA \\ Guido F. Smoorenburg, Cochair \\ Hearing Research Laboratories, University Hospital, Utrecht F02504, 3508GA Utrecht, The Netherlands
}

Chair's Introduction-7:55

Invited Papers

8:00

3aNSb1. The results of a unique Nordic HAKK interlaboratory REAT comparison. Torben Poulsen (Dept. of Acoust. Technol., Tech. Univ. of Denmark, DK-2800 Lyngby, Denmark)

The sound-field requirements in the hearing protector standard ISO 4869-1 are under discussion at present and are likely to be changed in a coming revision of the standard. The Nordic HAKK group comprises representatives from the hearing protector industry, labor protection institutes, audiological clinics, and universities. The test facilities at the laboratories of the members of the Nordic HAKK group cover various ways to fulfill the sound-field requirements of the ISO standard. Therefore, a round robin test was initiated with the aim of determining — and comparing between laboratories - the attenuation of two hearing protectors of the muff type and one of the plug type. The measurements were performed during the meetings of the group and with the group members (min. ten) as test subjects, thus permitting one group of subjects to be used by all laboratories. A statistical analysis of the results is in progress at the time of submission of this abstract. All factors (laboratory, protector-type, test-frequency, test-subject) are significant.

\section{8:20}

3aNSb2. Experience with a new ANSI standard for measuring the real-ear attenuation (REAT) of hearing protectors (S12.6-1997). Elliott H. Berger, Ronald W. Kieper, and Donald L. Peyton (E. A R Hearing Protection Products, 7911 Zionsville Rd., Indianapolis, IN 46268-1657, eberger@compuserve.com)

It has become increasingly apparent during the past 20 years that laboratory-measured values of hearing protector attenuation obtained in conformance with ANSI standards (S3.19-1974, S12.6-1984) bore little correspondence to those obtained by workers in actual occupational settings. The development of a new standard, ANSI S12.6-1997, Methods for Measuring the Real-Ear Attenuation of Hearing Protectors, addressed this problem. The new standard includes both experimenter-supervised fit and subject-fit methods. The latter method, designated as method B, requires the use of audiometrically experienced subjects who are naïve in the use of hearing protection. This method is intended to approximate the upper limits to the attenuation that can be expected for groups of occupational users. It yields mean attenuation values, more so for earplugs than earmuffs, that are substantially lower, and standard deviation values that are higher than previously found using ANSI standards. This paper will describe the new S12.6, present method-B test data, compare and contrast the standard to the existing related ISO standard (4869-1:1990), and review the results of ongoing studies designed to evaluate aspects and assumptions of the Method-B protocol. The problems inherent in obtaining and utilizing test panels of inexperienced hearing protection wearers, as well as observations gleaned from working with those subjects for in excess of 30 evaluations, will also be examined.

8:40

3aNSb3. Alternative field methods of measuring hearing protector performance. John R. Franks (Bioacoustics and Occupational Vib. Section, NIOSH, MS C-27, 4676 Columbia Pkwy., Cincinnati, OH 45226-1998)

Real-world tests of hearing protector attenuation are scarce. This study evaluated data from three new field-test methods as compared to the subject-fit data from Method B of ANSI S12.6-1997 for the same protector. The new field-test methods were: fit-check headphone (FCH) method [K. L. Michael, Spectrum 15, Suppl. 1, 24 (1998)], modified fit-check in sound-field (MFCSF) method, and modified bone conduction loudness balance (MBCLB) method [T. W. Rimmer and M. J. Ellenbecker, Appl. Occup. Environ. Hyg. 12, 69-75 (1997)], all of which can be administered in small audiometric booths such as are commonly found in industry. Twenty audiometrically competent subjects naive to hearing protector use were tested in the laboratory and for the three field-test methods. The earplug tested was the EAR Express Pod Plug. The results showed that the FCH method and the ANSI A0S12.6 subject-fit method produced similar attenuation levels. The MFCSF method showed decreased attenuation levels at 250 and $500 \mathrm{~Hz}$ while the MBCLB attenuations were elevated at 250,500, and $1000 \mathrm{~Hz}$ due to the occlusion effect of the HPD. The results suggest that the FCH method was the most feasible and reliable method for field testing, although it is limited to earplugs. [Portions of work supported by the U.S. EPA IA 75090527.] 
3aNSb4. The attenuation of combinations of ear muffs and ear plugs. Guido F. Smoorenburg (Hearing Res. Labs., Univ. Hospital Utrecht F.02.504, P.O. Box 85500, 3508GA Utrecht, The Netherlands, g.smoorenburg@kmb.azu.nl)

In a number of situations, a single hearing protector (earplug or earmuff) may not provide sufficient hearing protection. This may concern, for example, shooting noise in indoor firing ranges, shooting instructors also outdoors, and jet-engine testing sites. Combinations of earplugs and earmuffs may then be considered. The protection provided by these combinations cannot simply be obtained by adding the attenuation figures provided for the plug and muff individually. A principal limit is set by bone conduction of the sound through the skull to the inner ear. In addition, the total attenuation may be affected by acoustic coupling of the two protectors. It will be demonstrated how to estimate the total attenuation using attenuation measurements of combinations of three earmuffs (Bilsom Comfort, Clark 19A, Peltor H7A) and three earplugs (Bilsom Form, Bilsom Soft, Willson EP100) for 16 subjects and data on bone conduction. The experiment was carefully designed, aiming at a direct comparison of the attenuation for the protectors individually and together, within one subject and with a minimum of replacement of the protectors. The evaluation was based primarily on subjective measurements (REAT method) but the earmuffs have also been measured using miniature microphones in the outer ear canal.

3aNSb5. Artificial heads versus miniature microphones for measuring active noise-reduction hearing protector performance. Thomas Fedtke and Utz Richter (Physikalisch-Technische Bundesanstalt, Bundesallee 100, D-38116 Braunschweig, Germany)

The results of sound attenuation measurements on hearing protectors with head and torso simulators (HATSs) are quite encouraging if suitable corrections for the bone conduction and the occlusion effect are applied. Yet, the real-ear-at-threshold (REAT) method is still the standardized and most often used procedure to get appropriate measures for the protection provided by passive earmuffs and passive earplugs. With the development of active noise reduction (ANR) hearing protectors, however, the REAT method fails because of the background noise generated by the electronic components of the devices. Furthermore, the limits and the efficiency of such devices cannot be tested with sound-pressure levels near the hearing threshold. Thus there is an urgent need for using either HATSs or probe microphones on humans for the measurement of the ANR performance of a hearing protection device. Attempts to determine the effective attenuation with a combination of REAT and artificial head measurements will be compared with the results of microphone in real ear (MIRE) measurements for several commercially available ANR systems, including noise cancelling headphones. Aspects of validity and uncertainty of the various procedures, as well as practical relevance and ethical considerations, will be discussed.

9:40-10:00 Break

\section{0:00}

3aNSb6. Amplitude-sensitive attenuating earplugs. Pascal J. F. Hamery and Armand L. Dancer (French-German Res. Inst. of Saint-Louis, 5 rue du General Cassagnou, BP 34, 68301 Saint-Louis Cedex, France, dancer@newel.net)

The most simple amplitude-sensitive attenuating earplug is made of a plate with one little hole that is inserted in a perforated plug (like Racal Gunfender). That hole presents an acoustic impedance which has essentially a viscous resistance and a nonlinear dependence on the particle velocity in its center [L. J. Sivian, J. Acoust. Soc. Am. 7, 94-101 (1935); U. Ingard and H. Ising, ibid. 42, 6-17 (1967)]. The dimensions of that hole have been optimized and a new configuration, i.e., an empty cavity with two perforated plates, has been designed. This "filter" of small dimensions can easily be fitted into different commercial perforated earplugs. To better understand the experimental results and optimize the acoustic nonlinear "filter" dimensions, a theoretical model has been developed. Moreover, an experimental setup is described which allows visualizing the particle velocity inside the cavity with a particle imagery velocity camera which helps to understand the actual behavior of such a nonlinear cavity.

\section{0:20}

3aNSb7. Implementation of adaptive digital ANR in hearing protectors. Herman J. M. Steeneken and Jan A. Verhave (TNO Human Factors Res. Inst., P.O. Box 23, 3769ZG Soesterberg, The Netherlands, steeneken@tm.tno.nl)

Present state-of-the-art personal hearing protectors with ANR are mainly based on analog control circuitry. The performance of these systems is fair to good; an additional attenuation for low frequencies of $10-25 \mathrm{~dB}$ is obtained. By using a digital control circuitry for feedback gain control and compensatory filtering, a similar performance can be obtained. Digital signal processing allows for real-time control of the system parameters. By using an adaptive algorithm, the system performance can be optimized for individual (acoustic) frequency transfer and for the spectrum of the specific noise to be suppressed. A prototype system has been developed, in which the feedback gain and the frequency response parameters are adaptively controlled. The system uses an (additional) probe to determine the stability of the feedback loop. Other features of digital ANR are: continuous monitoring of the noise dose, transparency at low noise levels in order to improve direct communications and warning signals, and advanced signal processing of speech signals from an intercom. Preliminary results obtained with an add-on system (attached to a standard earmuff) will be presented and compared to the performance of 20 commercially available systems. 
3aNSb8. Use of the Zwicker method to predict signal audibility in noise with hearing protection devices. Martin Liedtke (BIA-Berufsgenossenschaftliches Inst. für Arbeitssicherheit, Alte Heerstrasse 111, D-53754 Sankt Augustin, Germany, M.Liedtke@hvbg.de)

Hearing protection devices (HPDs) can decrease perception of acoustic warning signals. Therefore, specific restrictions are given for the use of HPDs in specific danger areas in Germany. For example, up until 1996, German traffic regulation did not permit use of HPDs while steering a vehicle in road traffic. Within investigations carried out in the 1980s, the audibility of track-layer warning signals was examined during exposure to typical noises and when HPDs were worn. For this purpose, differences in audibility were determined on the basis of the Zwicker method (ISO 532, Method B). As a result, a procedure to predict signal audibility with HPDs was available to select appropriate HPDs for specific situations at work. A special procedure to be carried out if HPDs have to be worn in road traffic was published in 1996 in Germany: A list of hearing protectors realizes a preselection; a final perception test at work under operation conditions carried out by the individual employee and the person responsible for safety indicates the suitability of the HPD. Recent investigations on level-dependent HPDs show that the level of signal audibility seems to depend on the transfer characteristic of the level-dependent HPD.

\section{1:00}

3aNSb9. Hearing protection in everyday practice-The gap between expert knowledge and the beliefs of the users. M. L. Barrenas (Dept. of Auiology, Sahlgrens Univ. Hospital, Gothenburg, Sweden)

Many well-informed workers do not use hearing protectors (HPs) as they are taught to. Also, there are differences between what workers know, believe, and do. HPs are used whenever "necessary"' and taken off when "the noise levels are low." Workers know when the noise is measured at their workplace. However, when there is no feedback from these measurements, the noise levels will be regarded as being "all right." Based upon their own noise rating scale, workers create their own norms for HP usage. By regarding their hearing as normal, even when they know their hearing is impaired, there is no incentive to use HP more often. The great disparity between the knowledge of the experts and the way workers use HP shows that the awareness of damage risk is low. Adaptation to noise and to hearing disability is tremendous! Thus, HP usage is determined by the worker's own noise rating scale, by exchange of opinions between workers, and by their belief of having normal hearing, rather than by the objective noise measurements, information about the need of hearing protection and audiograms. Therefore, the hearing conservation procedures must be renewed according to modern pedagogic principles starting from the perspective of the users, not the experts.

\section{Contributed Papers}

\section{1:20}

3aNSb10. Acoustic modeling and measurements of hearing protectors for impulsive noise. Samir N. Y. Gerges and Erasmo F. V. Miranda (Federal Univ. of Santa Catarina (UFSC), Mech. Eng. Dept., Lab. Noise and Vib., Cx.P.476, Florianopolis, SC, Brazil, gerges@mbox1.ufcs.br)

REAL is the world-wide common method used for the hearing protector attenuation measurement in accordance with ISO 4869 and ANSI S12.6. This method is not suitable to determine the attenuation of the peak level of impulsive noise, because it measures the attenuation subjectively at low levels of a steady-state sound near the threshold of hearing. Alternative low cost and quick methods are required for assessing hearing protector attenuation for impulsive noise. In this paper a numerical model based on the finite-element method (FEM) for the impulsive noise attenuation prediction of the hearing protector is presented. The insertion loss of the peak level in the time domain is calculated and compared with laboratory measurements.

\section{1:40}

3aNSb11. A rating system in search of hearing protectors. William J. Murphy and John R. Franks (Bioacoustics and Occupational Vib.
Section, NIOSH, MS C-27, 4676 Columbia Pkwy., Cincinnati, OH 45226-1998)

Previous methods for rating the noise reduction of hearing protection devices (HPDs) have determined the mean attenuation and standard deviations at several octave-band frequencies using real-ear attenuation at threshold (REAT) measurements. Typically, some fraction of the standard deviation is subtracted from the mean attenuation to estimate an assumed protected value for a given percentage of the population. The subtractive correction assumes normally distributed numbers that are linear and not logarithmic. Data from several HPDs were analyzed with a statistical approach which first determined the A-weighted noise reduction for individual measurements. The cumulative distribution of A-weighted noise reduction (CDNRA) was then fit with a combination of two logistic regression curves. The Pearsons correlation coefficients for the model and the data were better than 0.99 for each of four devices analyzed. The CDNRA method yielded reasonable results from data collected from wellfit and poorly fit hearing protectors. In contrast, the NRR method would yield negative NRR values for poorly fit protectors. This paper describes the CDNRA method and looks at the results for an interlaboratory test of four HPDs, as well as additional data collected in the NIOSH Hearing Protector Laboratory. [Portions of work supported by the U.S. EPA IA 75090527.] 


\title{
Contributed Posters
}

These posters will be on display in the Poster Gallery from Thursday to Friday, 18-19 March. Authors will be at their posters from 2:00 p.m. to 4:00 p.m. on Thursday, 18 March.

3aNSb12. Four protectors in search of a rating system. William J. Murphy, John R. Franks, Dave A. Harris (Bioacoustics and Occupational Vib. Section, NIOSH, MS C-27, 4676 Columbia Pkwy., Cincinnati, OH 45226-1998), and Jennifer L. Johnson (USA MEDDAC, Hearing Conservation, Ft. Hood, TX 76544)

The EAR Classic, V-51R, Howard Leight Max-1 and EAR Express Pod insert earplug hearing protection devices (HPDs) were tested at the NIOSH Hearing Protector Laboratory using real-ear attenuation at threshold (REAT) according to ANSI S12.6-1997 Method B subject-fit (SF) and ANSI S3.19-1974 experimenter-fit (EF) methods. Several subject panels were created from the NIOSH subject pool which permitted comparisons to the manufacturer's REAT data published on the device's label. For the Classic, NIOSH data agreed with the manufacturer's data. However, for the other devices, the manufacturer's data typically produced higher noise reduction ratings (NRRs) than the NIOSH data. The subject-fit NRR, NRR(SF), results were 17, 1, 12, and $10 \mathrm{~dB}$ for the Classic, V-51R, Max and Express, respectively. The REAT variances for the Bilsom V-51R data and the Howard Leight Max were significantly smaller than the NIOSH variances. The NIOSH recommendation that SF data, measured according to ANSI S12.6-1997, be used to rate the performance of a hearing protector, is supported by these results. Application of the NIOSH and OSHA derating schemes to EF NRR either overestimated or underestimated the NRR(SF). [Portions of work supported by the U.S. EPA IA 75090527.]
3aNSb13. Effects of cup, cushion, headband force, and foam lining on the attenuation of earmuffs. Paulo Henrique $T$. Zannin (Dept. Engenharia Mecanica, Univ. Fed. Parana, Centro Politecnico, Curitiba, PR 81531-990, Brazil)

The essays were undertaken using an artificial head and a diffuse sound field, according to DIN ISO 4869-1. Increasing the cup mass improved the noise attenuation by $17 \mathrm{~dB}$ at $100-300 \mathrm{~Hz}$. The coherence function between cup vibration and sound pressure inside the cup has shown that a great amount of sound energy is transmitted to the inside of the cup, at low frequencies. The foam lining of the cup clearly attenuated the sound in the frequency between 2000 and $8000 \mathrm{~Hz}$. Increasing absorbent mass improved attenuation because resonances could be damped. The attenuation measured with a complete earmuff, and the same without the cushion, have shown that sound leakage occurs at frequencies ranging from 100-2000 Hz. The vaseline coating between the skin and the cushion improve the attenuation by less than $5 \mathrm{~dB}(100-200 \mathrm{~Hz})$. As the headband force increases to a maximal deformation of the cushion, attenuation reaches $20 \mathrm{~dB}$ at $200 \mathrm{~Hz}$. The increase in the headband force is, however, a limiting factor in the use of the earmuff, as it becomes too uncomfortable. The same conclusion applied to the increase in the mass of the cup.

WEDNESDAY MORNING, 17 MARCH 1999

ROOM H1028, 8:00 A.M. TO 12:00 NOON

\section{Session 3aNSc}

\section{Noise and Physical Acoustics: Experience with Noise Prediction II}

\author{
Karl-Wilhelm Hirsch, Cochair \\ Institut für Lärmschutz, Arnheimer Strasse 107, 40489 Düsseldorf, Germany \\ Keith Attenborough, Cochair \\ Faculty of Technology, The Open University, Milton Keynes MK7 6AA, UK
}

Invited Papers

\begin{abstract}
8:00
3aNSc1. Experiences with high-speed algorithms for full-field predictions of long-range sound propagation in the atmosphere. Martin West and Robert A. Sack (Univ. of Salford, Salford M5 4WT, UK)

Algorithms based on a parabolic equation (PE) solution of the Helmholtz equation have in the last few years become popular because of their precision and robustness, as well as their flexibility in dealing with a wide range of ground boundary conditions and meteorological conditions. PE solutions previously had to be performed on powerful workstations, but with recent advances in personal computer (PC) hardware it is possible to obtain limited predictions on more modest computers. The new algorithms being developed by this group have the potential to increase computational speeds of standard PE algorithms by up to two orders.
\end{abstract}


3aNSc2. The development of prediction models to control noise levels around UK firing ranges. Geoff Kerry and Martin West (Univ. of Salford, Salford M5 4WT, UK)

Since 1981, many UK firing ranges have used computer-based prediction models to control noise levels in the surrounding countryside. These models take into account local surface conditions and meteorology to provide peak noise level contour maps over distances of up to $30 \mathrm{~km}$. A feature of these models is that peak noise level maps can be produced within a few minutes of the input of meteorological data obtained directly from radiosonde ascents or from mesoscale models. Several new prediction models have been developed at Salford over the years, taking advantage of the ever increasing power of personal computers to improve accuracy and user input and output facilities. This paper will discuss the model developments, including the meteorological preprocessor, illustrate some of the techniques adopted to improve accuracy and speed, and outline future improvements.

\section{8:40}

3aNSc3. Measurements and computations of shooting noise. Part 1: Muzzle noise. Erik M. Salomons and Frank H. A. van den Berg (TNO Inst. of Appl. Phys., Stieltjesweg 1, 2600 AD Delft, The Netherlands)

The accuracy of a model for propagation of shooting noise is investigated by comparison with experimental data for muzzle noise and detonation noise of several weapons, over ranges up to $15 \mathrm{~km}$. The caliber of the weapons ranges from 7.62 to $155 \mathrm{~mm}$. The model is based on a database with values of the transmission loss computed with the parabolic equation method for a set of 27 atmospheric sound speed profiles, three ground types, and many different source-receiver configurations. For the comparisons between the model and the measurements, sound-speed profiles are selected from a set of 27 profiles, based on experimental profiles computed from meteorological data collected during the measurements. In general, good agreement is obtained between measured and computed sound levels and spectra. It is found that sound propagation over water yields considerably higher levels than sound propagation over grassland does. Situations with heterogeneous grounds are investigated as well, e.g., consisting of a grass area and a water area. [This work was coordinated and financed by the Dutch Ministry of Defence, Defence Co-ordinator Physical Planning and Environmental Affairs (MODNL-CROMD).]

\section{Contributed Papers}

\section{9:00}

3aNSc4. Measurements and computations of shooting noise. Part 2: Bullet noise. Frank H. A. van den Berg and Erik M. Salomons (TNO Inst. of Appl. Phys., Stieltjesweg 1, P.O. Box 155, 2600 AD Delft, The Netherlands)

Due to the supersonic speed of the bullet, a shock wave is generated (sonic boom). A method has been developed to compute the generation and propagation of bullet noise on the basis of the geometry of the bullet and its speed. This model also holds for bullet paths of finite length with decreasing bullet speed. The accuracy of this model is investigated by comparison with experimental data for propagation of bullet noise of several weapons, over ranges up to $7 \mathrm{~km}$. The caliber of the weapons ranges from 5.56 to $120 \mathrm{~mm}$. The measurements indicate that nonlinear effects are only important for the first few hundred meters from the bullet path. This is explained by a coherence loss, caused by propagation through the atmosphere. When corrected for this loss, the measurements and calculations of bullet noise compare reasonably well. [This work was coordinated and financed by the Dutch Ministry of Defence, Defence Co-ordinator Physical Planning and Environmental Affairs (MODNL-CROMD).]

\section{9:20}

3aNSc5. Calculation of aircraft sound propagation on airports via determination of ground impedance. Reinhard Blumrich (DLR, Institut für Physik der Atmosphäre, D-82234 Weßling, Germany, reinhard.blumrich@dlr.de) and Jürgen Altmann (Ruhr Universität Bochum, 44780 Bochum, Germany)

In the framework of acoustic and seismic monitoring of airports for verifying disarmament or peace-keeping agreements, the propagation of sound emitted by aircraft was investigated. This propagation is strongly affected by the porous ground. A determination of the ground surface impedance allows one to calculate the attenuation and phase shift with distance and height. In the course of aircraft signal measurements at the military airport at Jever, Germany, the surface impedance of the grasscovered ground was determined from c. $60 \mathrm{~Hz}$ to $2.2 \mathrm{kHz}$ by measurements of complex transfer functions using (quasi-)white noise and a numerical inversion of the spherical wave reflection factor. A threeparameter impedance model was fitted to the measured impedance. By means of the ground impedance propagation calculations are carried out.
In the case of the transfer function measurement signals, the calculated spectra agree very well with the actually measured ones. In the case of the jet aircraft, the simulated spectra fit to those of the measured signals only in general appearances of amplitude, and not in phase. This is probably due to an inaccurate localization of the sound source, its extension and directivity. Wind and temperature gradients were not considered.

$$
\text { 9:40-10:00 Break }
$$

\section{0:00}

3aNSc6. Forecasts of sound propagation by a mesoscale atmospheric model. Svenn O. Haugland (Geophysical Inst., Univ. of Bergen, Allegt. 70, 5018 Bergen, Norway) and Lars R. Hole (Norwegian Defence Construction Service (NDCS), Oslo mil/Akershus, 0015 Oslo, Norway, larsr@gfi.uib.no)

A mesoscale atmospheric model has been used to simulate local wind and temperature profiles in an area with hilly terrain in eastern Norway. Simulated wind and temperature profiles have been used to determine the local sound velocity profiles. The predicted profiles were used as input in an acoustic model. Meterological measurements were carried out in conjunction with measurements of propagation of low frequency $(<100 \mathrm{~Hz})$ impulse sound. The predicted atmospheric profiles are compared with profiles measured by meteorological towers and a tethered balloon launched at the site.

\section{0:20}

3aNSc7. Meteo-BEM: A new hybrid approach to predict noise barriers' efficiency with meteorological effects, theory, and comparison with experimental results. Eric Premat (ENTPE, DGCB CNRS URA 1652, 2 rue Maurice Audin, 69518 Vaulx-en-Velin Cedex, France) and Yannick Gabillet (CSTB, 38400 Saint-Martin d'Heres, France)

In a complex outdoor environment, meteorological factors have been found to affect the sound field. On one hand, boundary element methods are particularly well adapted to calculate the sound pressure above complex boundaries (various absorbing properties and shapes) in a homogeneous atmosphere. On the other hand, a few models have been recently developed to describe the acoustic propagation in inhomogeneous media 
(normal modes, residue series, parabolic equation, fast field program ....). Meteo-BEM is a new hybrid formulation taking advantage of the power of the boundary element methods, and including meteorological effects using as the appropriate Green's function these recent propagation models in inhomogeneous media. In this paper, the theory of BEM, together with sound propagation in inhomogeneous media, is briefly recalled. The new hybrid model is then presented. The case of a rigid thin-noise barrier on a flat ground under a known sound-speed gradient condition is studied. Results from Meteo-BEM are then compared to measurement data for validation. It is shown that this new model allows one to calculate accurately the sound field in a complex outdoor environment and offers promising further developments.

\section{0:40}

3aNSc8. Assessing changes in the noise environment from military training ranges: A case study in forensic acoustics. George A. Luz and Nelson D. Lewis (U.S. Army Ctr. for Health Promotion and Preventive Medicine, 5158 Blackhawk Rd., Abereen Proving Ground, MD 21010-5422)

In response to litigation alleging that an Army installation was making more noise in land adjoining tank and artillery ranges in 1997 than when the owner had purchased the land in 1969, the past weapons noise environment had to be reconstructed from available data. The results, which documented the changes in the training noise environment from 1971 to 1984 to 1997 , demonstrated the long-term benefits of the U.S. Army Construction Engineering Research Laboratories' BNOISE computer model. Also discussed is (1) the use of automated noise monitoring data to validate "busy day" weapons noise contour maps and (2) the potential for cost savings by combining environmental noise modeling with air, water, and solid waste pollution.

\section{1:00}

3aNSc9. Moving point source-A simple model for predicting statistical sound levels in the neighborhood. Edelbert Schaffert and Stefan Becker (BesB GmbH Berlin, Undinestr. 43, 12203 Berlin, Germany)

When predicting the noise produced by moving sound sources (e.g., vehicles, airplanes, etc.) sound-level statistics can be helpful to describe the situation. However, the common sound models only yield the average sound level, and under special circumstances, also the maximum sound level. The calculation model moving sound source also gives sound level and the level-versus-time diagram. For the calculation, first the paths of the sound sources have to be digitalized and divided into parts. Each part will be represented as a point sound source which is active during a certain time slot, which again is based upon the velocities of the moving objects. Sound propagation from all point sources to the point of emission is calculated by using ISO or equivalent standards. This leads to the time versus level diagram and (when the steps from one to another point source are small enough) to the desired statistical levels. The described model, named the moving sound source, has no limitations. It could be easily integrated in commercial sound propagation calculation programs. It was successfully used for the approval of a new racetrack in Germany. Also, the new DIN 45684 (noise of small aircraft) is based on it.

11:20

3aNSc10. Track dispersion in aircraft noise modeling. Rudolf Buetikofer and Stefan Pluess (EMPA, CH-8600 Duebendorf, Switzerland)

Aircraft noise calculations based on clusters of single flight events are compared to results from a set of average flight paths. For the situation of Zurich airport, the aircraft noise was calculated with two different methods for the data input to the noise calculation program. The traditional method consists of defining a main track and several side tracks, each associated with a specific percentage of traffic. Further, an average climb profile was defined for each aircraft type. The definition of these average flight paths is based on the analysis of radar information from the airport. The new method selects for a given aircraft and departure (or landing) procedure from all available radar data sets a representative sample of, e.g., 100 flights, including different seasons, different companies, and different take-off weights. For each individual (real) flight the aircraft noise is calculated and the results are summed up according to the flight statistics. The price is an increase of computation load by a factor of 20 , the benefit is a more representative result especially for regions with curved flights where the tracks diverge over a large area.

\section{1:40}

3aNSc11. Computational methods for designing a silator-A lowfrequency resonator element. Norbert Kalus (Dept. of Math.-Phys.-Chem., TFH Berlin Univ. of Appl. Sci., Luxemburgerstr. 10, D-13353 Berlin, Germany, kalus@tfh-berlin.de)

The silator is a vacuum bubble resonator element which is used as a noise control device and has a low-frequency performance. It consists of an evacuated, lens-shaped, convex cap of sheet metal. The capsule volume can be made as small as desired, since, because of the vacuum inside, there is no air stiffness. The effect of a silator is comparable to that of a Helmholtz resonator, but only a hundredth of the latter's installation volume is needed. Broadband noise control can be achieved by a set of silators tuned to different frequencies. The principles of this silator technology in aeroacoustics were established by Bschorr and Laudien in 1992. The resonant characteristics of such a device are very sensitive to its geometrical properties. The plasticity of the material, the metal forming, and the remaining stresses in the convex cap also influence its effectiveness. The major development problem lies in the extremely small manufacturing tolerances. The tolerances decrease with increasing size and frequency. In this paper, computational methods and results are presented to yield the final design of the silator's shape with a very low resonant frequency. The calculations were performed using the finite-element program MSC/NASTRAN for Windows. 


\title{
Session 3aNSd
}

\author{
Noise: Aerodynamic Noise of Vehicles \\ Bernd Barsikow, Cochair \\ Akustik Data Engineering Office, Kirchblick 9, D-14129 Berlin, Germany \\ Paul R. Donovan, Cochair \\ General Motors Proving Grounds, 24-NVL, Milford, Michigan 48380-3726, USA
}

Chair's Introduction-8:55

Invited Papers

9:00

3aNSd1. Characteristics of the Aeolian tone generated from cylinders in conjunction with pantograph noise control. Hajime Fujita (Dept. of Mech. Eng., Nihon Univ., 1-8 Kanda Surugadai, Chiyoda-ku, Tokyo 101-8308, Japan) and Akio Sagawa (Railway Tech. Res. Inst., Maibara, Shiga 521-0013, Japan)

Aerodynamic sound generated from cylindrical objects, such as in pantographs of trains, is a prime noise source in high-speed vehicles. Characteristics of the aerodynamic sound generated from two-dimensional cylinders are studied experimentally in order to contribute to the prediction and control of high-speed vehicle noise. The effect of the angle of inclination to the mean flow of a circular cylinder, and the effect of the angle of attack of a square cylinder, to the Aeolian tone generation are studied quantitatively, and angle ranges for low noise generation are found in the subcritical Reynolds number range. The relative importance of the cylinder surface pressure fluctuation and its spanwise correlation length to the Aeolian tone generation is revealed. Experiments are extended to the super-critical Reynolds number range for a circular cylinder normal to the mean flow. In the beginning of the surface boundary layer transition, the Strouhal number suddenly jumps up to about twice as much and the Aeolian tone level decreases suddenly about $20 \mathrm{~dB}$. It is found that the decrease of the Aeolian tone level is mainly due to the decrease of the cylinder surface pressure fluctuation. Application to recent pantograph development is discussed.

\section{9:20}

3aNSd2. Reducing the sound emission of TRANSRAPID by decreasing the aerodynamic sound sources. Klaus-Peter Schmitz and Hans-Gerd Runde (Industrieanlagen-Betriebsgesellschaft mbH-Magnetbahn-Versuchsanlage-Hermann-Kemper-Straße 23, D-49762 Lathen, Germany, klaus-peter.schmitz@tm.iabg.de)

With the support of the BMBF and in commission of the MVP, the IABG pursues the TRANSRAPID test facility. The TRANSRAPID vehicle TR07 started in 1989 with a very low sound emission. A passby level of $94 \mathrm{~dB}$ was measured in a distance of $25 \mathrm{~m}$ (free propagation and without soundshields) and a speed of $400 \mathrm{~km} / \mathrm{h}$. First investigations with a microphone array pointed out a very calm upper part of the car body and some sound sources in the region of the bow as well as in the region of the levitation and guidance system (LGS). These results showed the low importance of the noise emissions caused by the turbulent boundary layer (TBL) as there was no sound emission found in the upper part of the vehicle. So the sound emission seemed to be caused by aerodynamic sound sources coming from discontinuities of the shape. To reduce the sound emission especially the shape of the bow was modified, the design of the covering plates of the LGS was optimized, and new inner coverings between the vehicle and the guideway were developed. All these changes have decreased the sound emission of the TR07; today a pass-by level of $88.5 \mathrm{~dB}$ is realized.

3aNSd3. Aerodynamic noise from vehicles on the Yamanashi Maglev Test Line. Masaaki Miyamoto, Mikio Yamazaki, Yasuyuki Goto (Linear Express Development Div., Central Japan Railway Co., Chuo-ku, Tokyo, Japan), Makoto Ueno (Railway Tech. Res. Inst., Chuo-ku, Tokyo, Japan), Yasuo Zenda, and Masanobu Iida (Railway Tech. Res. Inst., Kokubunji-City, Tokyo, Japan)

This paper is a report about aerodynamic noise from Maglev vehicles running at an ultra high speed. Maglev running tests have been carried out on the Yamanashi Maglev Test Line and $550 \mathrm{~km} / \mathrm{h}$ was recorded in December 1997. The noise produced when Maglev vehicles run at high speed consists of almost only aerodynamic noise because of the levitated running of Maglev vehicles. Two types of nose shapes were developed for Yamanashi test cars in order to considerably reduce air drag and aerodynamic noise. The noise measured when Maglev vehicles running on the viaduct at the velocity of $500 \mathrm{~km} / \mathrm{h}$ will be reported in this paper. Also the rate of increase of noise to velocity and the source of noise of running Maglev vehicles will be reported. In general, it is known that when a high-speed train enters a tunnel, a compression wave is generated ahead of the train and a pulse wave radiates from the exit of the tunnel, and an air pressure booming noise is generated in some cases. This air pressure booming noise is successfully suppressed by setting a hood at the tunnel entrance in this test line. 
3aNSd4. Sound radiation from flow-excited, elastically supported rectangular panels. L. Mongeau, J. Fazio (School of Mech. Eng., Purdue Univ., 1077 Herrick Labs., West Lafayette, IN 47907-1077), and N. Vlahopoulos (Univ. of Michigan, Ann Arbor, MI 48109-2145)

The objective of this study was to evaluate the influence of the viscoelastic properties of glass run weather seals on the interior noise radiated by vehicle window panel vibrations. The vibrational response of a flow-excited rectangular aluminum panel supported on all four edges by a fixture hosting a glass run rubber sealing system was measured in a low-speed wind tunnel. The panel was attached to an acoustically treated enclosure located underneath the wind tunnel test section. The resonance frequencies, damping coefficients, and mode shapes of the panel were measured following established modal analysis procedures. Two different sealing systems were investigated to study the influence of seal compliance and damping factor on the sound transmission loss of the elastically supported panel. The experimental results were compared to predictions obtained using finite-element and boundary element numerical methods. The sensitivity of the radiated sound level to variable seal properties was evaluated. The possible applications of these results to quiet vehicle seal design were considered.

\section{0:20-10:40 Break}

\section{0:40}

3aNSd5. Wind tunnel noise measurements on full-scale pantograph models. Thomas Lölgen (Dept. of Acoust., Res. and Technol. Ctr., Deutsche Bahn AG, Völckerstr. 5. 80939 München, Germany)

Noise reduction of pantographs is of special importance for the operation of high-speed trains, since the pantograph noise cannot be treated with passive counter measures like sound barriers. Therefore the noise emission of pantographs was investigated in an acoustically treated wind tunnel for air speeds up to $400 \mathrm{~km} / \mathrm{h}$. The aims were to determine the noise reduction potential of pantographs, to investigate their aerodynamic behavior, to validate existing theoretical models, and to compare measurement techniques and wind tunnels. The acoustic measurements were carried out using a microphone array system, a parabolic antenna as well as normal microphones. Two pantographs, which are in service today in Germany and Japan, were used as references. Two full-scale models of high-speed pantographs were tested in several configurations. The achieved noise reduction of the models compared to the reference pantographs confirmed the theoretical results.

\section{Contributed Papers}

\section{1:00}

3aNSd6. Measurements of sound radiated by turbulent wall jets past singularities. Marc C. Jacob (LMFA, UMR CNRS 5509, Ecole Centrale de Lyon BP 163, 69131 Ecully Cedex, France), Alain Louisot (Métraflu, 69130 Ecully, France), and Sylvie Guerrand (Direction de la Recherche et de la Technologie, SNCF, 75379 Paris Cedex 08, France)

Sound radiated by high-speed turbulent wall jets past backward facing steps or shallow cavities is investigated experimentally. Both aerodynamic (laser Doppler and hot wire anemometry) and acoustic measurements (farfield directivity, spectra, and source localizations) are performed. Source localizations show that a backward facing step is a major sound source. Far-field measurements show that it has a strong upstream directivity and radiates in the medium frequency range. Aerodynamical results and an acoustic parametric study suggest that the source is due to the diffraction of the separated shear layer eddies by the sharp edge of the step: this is qualitatively confirmed by existing diffraction models. A shallow cavity (with length exceeding seven times the depth) has a strong downstream and upstream directivity: it can be regarded as a superposition of two acoustically independent sources, a backward facing and an upward facing step. In particular for such cavities and for Reynolds numbers (based on cavity depth) ranging from 40000 to 470000 no feedback mechanism and no cavity resonances are observed. [Work supported by the French Ministère des transports.]

\section{1:20}

3aNSd7. Boundary-layer noise from high-speed vehicles: Theory and measurement. W. F. KingIII, B. Barsikow, and M. Klemenz (akustik-data Eng. Office, Kirchblick 9, D-14129 Berlin, Germany, akustik_data@compuserve.com)

Wayside noise emitted by the turbulent boundary layer (TBL) on a moving vehicle represents its minimal acoustic radiation. When wayside noise levels for aerodynamically clean vehicles (particularly maglev ones) begin to approach within $3 \mathrm{~dB}$ of the TBL noise level, further abatement measures become ineffectual. Knowledge of this lower bound to wayside noise is important to avoid expenditures for useless attempts at further lowering sound levels. The acoustic energy of TBL noise per unit area is low, but its cumulative contribution on a long vehicle could be significant. The present paper comprises two parts. One is a semi-empirical theory of TBL noise. Although Lighthill's acoustic analogy correctly describes TBL noise, this equation cannot be solved without knowledge of the (as yet) unknown source terms. Furthermore, the fluctuating density, the desired solution, appears on both sides of the equation. Hence, a model approach becomes necessary. The present offering is based upon extrapolated parameters describing the burst-sweep cycle within a flat-plate TBL. The second part of the paper summarizes results of array measurements made above the smooth roof of the maglev vehicle Transrapid 07 at speeds up to $400 \mathrm{~km} / \mathrm{h}$. Predictions show reasonable agreement with measurements.

\section{1:40}

3aNSd8. The relationship between the power level and different parameters of a single vehicle noise. Antonio Reig, Esteban Gaja, Jose Luis Manglano, and Salvador Sancho (ETSII Univ. Politecnica Valencia, Cno Vera s/n, 46022 Valencia, Spain)

This paper is concerned with the description of various factors, such as velocity, gears, and type of vehicle affecting the power level of a vehicle noise; a resume of this dependence will be offered. This work has been done in the Universidad Politecnica de Valencia (Spain) by the Laboratorio de Ingeniera Acoustica within a Thesis Project, with the aim to develop a predictive mathematical model of the urban traffic noise. Measurements were taken with an analyst Bruel mod. 2236, which enables one to obtain power levels in third octave bands by a real-time spectral analysis. The measurements being used are extensively explained in this paper, in particular that special care was taken to avoid noise from other sources that could interfere with the results. The reason why the power level was chosen as the first stage of this study of traffic noise, starting from the single vehicle as the point source, was to comply with the methodology established in the second part of standards ISO 9613, leaving for a second stage the propagation of the urban traffic noise. The final results show the great correlation between speed of the vehicle and power level. 
have a relatively small transmission loss. Due to the very strong flow

3aNSd9. Experimental investigations of surface pressure fluctuations related to car interior noise. B. Brähler, I. U. Borchers, L. Schauwecker (Dept. of Vibroacoutics, Daimler Benz Aerosp. Dornier $\mathrm{GmbH}$, Friedrichshafen, Germany, Boris.Braehler@dbag.fdh.daimlerbenz.com), and V. Mellert (Universität Oldenburg, Oldenburg, Germany)

Due to the progress in the reduction of engine noise and tire/road noise for cars, aerodynamically induced noise becomes increasingly important for car interiors and for speeds above $120 \mathrm{~km} / \mathrm{h}$. The aerodynamically induced noise is mainly caused by flow separations which may lead to highly intensive surface pressure fluctuations forcing the car housing to vibrate and to radiate. This is the case especially for the windows, which separations behind both the A pillar and the rearview mirror the side windows play a major role as aeroacoustic noise sources in the interior of automobiles. In this study the flow around the side windows of a production car, where there is nearly no vortex separation on the A pillar, is investigated. Therefore, the influences of the A pillar and rearview mirror could be studied independently by generating each source separately. For different configurations measurements of the hydrodynamic pressure fluctuations next to the side window were performed. Dependent on the spatial distribution of the pressure fluctuations significant changes for the car interior noise may be expected. The experimental results suggest a large potential of further reduction of car interior noise due to an aeroacoustic optimization of the flow.

WEDNESDAY MORNING, 17 MARCH 1999

ROOM H110, 8:20 A.M. TO 12:20 P.M.

Session 3aNSe

Noise: IEC61672-The New International Standard for Sound Level Meters

\author{
Alan H. Marsh, Cochair \\ DyTec Engineering, 5092 Tasman Drive, Huntington Beach, California 92649, USA \\ Hans O. Finke, Cochair \\ Opferkamp 12, D-38110 Braunschweig, Germany
}

Invited Papers

8:20

3aNSe1. Introductory remarks. Alan H. Marsh (DyTec Engineering, Inc., 5092 Tasman Dr., Huntington Beach, CA 92649) and Hans-Otto Finke (Opferkamp 12, D-38110 Braunschweig, Germany)

The new International Standard (IEC 61672, sound level meters) is being developed by Working Group 4 for IEC/Technical Committee 29, Electroacoustics. The standard will be published by the International Electrotechnical Commission (IEC). It amalgamates and extends the specifications and test procedures that were given in IEC 60651:1979 for conventional instruments that display sound levels with exponential time weighting and IEC 60804:1985 for integrating-averaging instruments that display time-average sound levels. A sound-level meter conforming to the specifications of IEC 61672 will provide the user with higher-quality measurements than previously available, especially for transient and short-duration sounds. This standard represents the culmination of years of effort by a dedicated team of experts from several countries. This special session will describe the acoustical and electrical characteristics that are required. All presenters of papers in this session are members of Working Group 4.

8:40

3aNSe2. Scope of the new standard. William V. Richings (3 Old Hall Ave., Duffield, Belper, Derbyshire DE56 4GN, UK)

The fundamental requirements for IEC 61672 have not changed. However, practical experience, advances in technology, and current regulations have been taken into account when developing the new standard. The type 0 accuracy category of IEC 651 is almost nonexistent because it is too specialized, while type 3 has been made redundant by the low cost of modern circuitry. Thus the new standard describes only two performance classes corresponding to much improved types 1 and 2 of the previous standard. Important new annexes give detailed procedures for conformance tests and for periodic checks. It is now recognized that the uncertainties of measurement are not negligible and realistic tolerance limits have been specified to include them. Another significant advance for the measurement of impulsive sounds is that a minimum linear operating range of $60 \mathrm{~dB}$ is specified for all instruments. Existing and draft European Community Directives call for peak C-weighted measurements and appropriate specifications are given. Requirements for immunity to electrostatic discharges and radio-frequency fields and for radio-frequency emission have been included to cover regulations in Europe and elsewhere. The environmental reference conditions have been changed to $23{ }^{\circ} \mathrm{C}$ and $50 \%$ relative humidity for consistency with the IEC microphone standards.

\title{
9:00
}

3aNSe3. Free-field response of sound level meters. Gaston Banget-Mossaz and Jean-Noël Durocher (Laboratoire National d'Essais, 75724 Paris, Cedex 15, France, Gaston.Banget@lne.fr)

Within specified ranges, sound level meters should be able to measure a frequency-weighted sound pressure level at a given point, regardless of the direction of sound incidence and the frequency of the sound. Acoustical characteristics of sound level meters are specified in draft IEC 61672 as a function of frequency for plane progressive sound waves of steady sinusoidal signals under free-field conditions. The free-field frequency response of a microphone in a specified reference direction is modified by (a) sound reflected 
from the case of the sound level meter, (b) the apparatus that supports the instrument under test, and (c) the frequency response of the system that processes the signals from the microphone. From the simulated influence of sound waves reflected from an instrument equipped with a microphone having a FLAT frequency response, it is shown that linear frequency steps should be preferred to logarithmic frequency steps for evaluation of reflection effects. Test signal frequencies are calculated from the quotient of the speed of sound by the distances from the reflection point on the case to the microphone reference point. Measurement results are shown and comments noted regarding the procedures in the proposed standard for testing acoustical characteristics.

9:20

3aNSe4. Frequency weightings and responses. Peter Hedegaard (Brüel \& Kjaer, 2850 Naerum, Denmark)

The first frequency weightings for sound-level meters were described in the 1940s in American and German standards. The well-known A, B, and C frequency weightings were originally designed to match the equal loudness contours described by Fletcher and Munson. These frequency weightings were realizable by simple passive electronic circuits. Even though the frequency weightings in proposed IEC 61672 are unchanged, their use is changed. Frequency weightings are now defined by their poles and zeros, and may be calculated to any degree of precison. Since 1961, other frequency weightings have been standardized for special purposes but are largely obsolete, along with the B weighting. Tolerance limits are discussed for the different performance classes and their changes from 1961 to the present. Wide tolerance limits for the high- and low-frequency ends of the frequency range are necessary to allow for different microphone sizes, manufacturing tolerances, and unattended operation outdoors. The wide limits may cause differences in measurement of sound levels if the sound has strong high- or low-frequency components. Some unsuccessful attempts to solve the problem are discussed. The new Z-weighting, FLAT frequency response, and the use of C-weighting for measurements of peak sound-pressure level, are discussed.

9:40

3aNSe5. Response to steady signals. Theodore J. Kuemmel (Quest Technologies, Inc., 510 S. Worthington St., Oconomowoc, WI 53066)

The level of a steady sinusoidal signal indicated by a sound-level meter should be linearly related to the level of the corresponding signal at the input. Small level-linearity errors over a wide range of input signal levels and signal frequencies are a powerful indicator of the ability of a sound-level meter to square and form the time integral of the sound-pressure signal properly. Errors in level-range controls as well as level linearity are specified in proposed IEC 61672 relative to a fixed reference level difference at the reference frequency for the reference sound-pressure level on the reference level range. At $1 \mathrm{kHz}$, the extent of the linear operating range is to be at least $60 \mathrm{~dB}$. The Instruction Manual is to state the nominal sound levels at the lower and upper boundaries of the linear operating ranges at specified frequencies. The new standard requires an instrument to have overload and under-range indicators. Level-linearity errors are to be within the tolerance limits up to the first indication of overload. Data will be presented to demonstrate that modern designs for sound-level meters can conform to the specifications for the response to steady sinusoidal electrical signals.

\section{0:00}

3aNSe6. Response to nonsteady signals. Ole-Herman Bjor (Norsonic AS, P.O. Box 24, N-3420 Lierskogen, Norway)

A sound-level meter should respond correctly to the frequency-weighted energy of the sound signal, no matter whether the signal comes from a pistol shot or a fan. Most sound signals of interest are transients, some with very short durations and high peak amplitudes. A major part of the proposed IEC 61672 standard describes requirements for the response of a sound-level meter to short duration signals of various levels and durations. Compared with the present standards for sound-level meters, the requirements for measurements of short-duration signals have been improved considerably. After considerable discussion, the Working Group decided to specify and test a sound-level meter with electrical toneburst signals of various durations as extracted from a steady $4-\mathrm{kHz}$ sinusoidal signal. The specifications apply to all kinds of sound-level meters; hence, they are given in terms of maximum F- and $\mathrm{S}$-weighted sound level and in terms of sound exposure level or time-average sound level. The response of the microphone is specified separately. Examples will be shown to demonstrate the ability of modern-design class 1 and class 2 sound-level meters to conform to the specifications of the proposed International Standard.

10:20-10:40 Break

\section{0:40}

3aNSe7. Peak C-weighted sound-pressure level. Hiroaki Takinami (Rion Co., Ltd., Kokubunji, Tokyo, Japan)

Peak sound pressure is the greatest absolute value of the instantaneous sound pressure occurring within a stated time interval. Peak sound-pressure levels are measured without exponential time weighting and hence are fundamentally different from maximum sound levels measured with time weighting F or S. Until the development of the new International Standard for sound-level meters in IEC 61672 , there was no internationally accepted specification for measurements of the peak level of nonsteady or transient sounds. For short-duration sounds, the value of the peak sound-pressure level depends on the frequency bandwidth of the measuring device; very short-duration signals require a wide bandwidth of uniform frequency response. To permit reproducible measurements of various short-duration sounds, IEC 61672 provides specifications for peak C-weighted sound-pressure levels. Frequency-weighting C has the most uniform response over the widest bandwidth of any internationally standarized frequency weighting. The specification requires that the peak C-weighted levels of a single cycle at $31,5 \mathrm{~Hz}, 500 \mathrm{~Hz}$, and $8 \mathrm{kHz}$ and positive and negative half cycles of $500-\mathrm{Hz}$ sinusoidal signals be measured within practical tolerance limits. Data will be shown to demonstrate conformance of instruments to the specification. 
3aNSe8. Environmental considerations for sound-level meters. Richard G. Tyler (Cel Instruments Ltd., 35-37 Bury Mead Rd., Hitchin, Herts SG5 1RT, UK)

A sound-level meter should operate as intended over a wide range of environmental conditions. To ensure this objective, IEC 61672 sets limits for the influence of atmospheric conditions and radio-frequency (rf) fields. A limit is also established for the rf emissions from a sound-level meter. Specifications in IEC 61672 for the influence of temperature and humidity on the performance of a sound-level meter are more stringent for class 1 instruments than corresponding specifications in existing standards. The extremes of the temperature-humidity range are reduced for class 2 instruments. Requirements for the effects of atmospheric pressure remain similar. New specifications were added for the effects of static discharges up to $8 \mathrm{kV}$ and for the influence of exposure to a moderate rf field. Limits for the influence on instrument performance of a given rf field, with carrier frequencies from $25 \mathrm{MHz}$ to $1 \mathrm{GHz}$, should satisfy most existing national and European Union requirements. Tests to demonstrate conformance with these requirements are specified in the proposed International Standard. Test results will be presented, as available, to demonstrate that modern designs for sound-level meters are able to conform to the environmental specifications in IEC 61672.

11:20

3aNSe9. Uncertainties of measurement and IEC 61672. Susan P. Dowson (Ctr. for Mech. and Acoust. Metrology, Natl. Physical Lab., Teddington TW11 0LW, UK)

No measurement is ever exact; there is always an uncertainty associated with it. In the past, international specification standards for the performance of acoustical instruments have not included uncertainties of measurement of the testing laboratory when specifying tolerance limits. However, to demonstrate conclusively whether or not a particular instrument conforms to the specifications of a standard, it is essential that these measurement uncertainties be taken into account. In a recent change of policy, the international committee responsible, IEC TC-29 "Electroacoustics," has decided that all its future standards must include uncertainties of measurement in the tolerance limits specified. This paper will discuss different types of measurement uncertainty and likely contributions to uncertainty budgets. It also explains the difficulties in accumulating the necessary data for inclusion in IEC 61672, and of incorporating the data into the tolerance limits. The reasoning behind the decision to quote a maximum permitted expanded uncertainty of measurement for the testing laboratory separately for each test will also be discussed. Examples will be presented of the determination of expanded uncertainties of measurement for acoustical and electrical signals and their application in assessments of conformance.

\section{Contributed Paper}

\section{1:40}

3aNSe10. Tolerances of " $A$ "-weighting at extreme frequencies. Alan D. Wallis and Christopher G. Rice (ISVR Univ. of Southampton, Southampton SO17 1BJ, UK, adwallis@compuserve.com)

Since IEC 123 (sound level meters) there has been an infinite negative tolerance permitted on the frequency weightings at the extremes of frequency. For example, the attenuation of A-weighting at $31.5 \mathrm{~Hz}$ was 39.2 $\mathrm{dB}$, with a tolerance of $\pm 5 \mathrm{~dB}$. At all frequencies below this, the tolerances are $+5 \mathrm{~dB}$ and minus infinity. A similar situation occurs at $8 \mathrm{kHz}$ and above. Thus a sound level meter which did not respond at all to signals below $31.5 \mathrm{~Hz}$ or above $8 \mathrm{kHz}$ may well meet the full requirements of the standard. In 1979, IEC 651 kept the same situation for a type 3 instrument, but changed the frequencies at which infinite negative tolerances were permitted to below $20 \mathrm{~Hz}$ and above $12.5 \mathrm{kHz}$ for a type 1 instrument. The August 1998 draft of IEC 61672-3CD also has infinite tolerances but now at $16 \mathrm{~Hz}$ and $16 \mathrm{kHz}$ for a class 1 and $20 \mathrm{~Hz}$ and $8 \mathrm{kHz}$ for a class 2. Many workers suggest that infinite tolerances should no longer be permitted, while others think that they are essential. This paper evaluates both sides of the argument and offers suggested tolerance figures.

\section{Invited Paper}

12:00

3aNSe11. Summary. Alan H. Marsh （DyTec Engineering, Inc., 5092 Tasman Dr., Huntington Beach, CA 92649)

The proposed International Standard, IEC 61672, for sound-level meters, differs significantly from the existing standards for such instruments. Existing standards include IEC 60651:1979 for conventional instruments that measure time-weighted and frequencyweighted sound levels and IEC 60804:1985 for integrating-averaging and integrating sound-level meters that measure time-average sound levels and sound exposure levels, respectively. The principal differences and improvements will be highlighted. The anticipated schedule leading to eventual publication by IEC will be indicated. 


\title{
Session 3aNSf
}

\author{
Noise: Turbomachinery Noise \\ Johan B. H. M. Schulten, Cochair \\ National Aerospace Laboratory (NLR), P.O. Box 153, 8300 AD Emmeloord, The Netherlands
}

\author{
Stewart A. L. Glegg, Cochair \\ Center for Acoustics and Vibration, Department of Ocean Engineering, Florida Atlantic University, Boca Raton, \\ Florida 33431-0991, USA
}

Chair's Introduction-9:05

Invited Papers

9:10

\begin{abstract}
3aNSf1. On the noise from turbomachinery and ducted fans-A review of prediction methods. Michel Roger (LMFA, UMR CNRS 5509, Ecole Centrale de Lyon, BP 163, 69131 Ecully Cedex, France)

Noise reduction of turbomachines and ducted fans is a major issue in aeronautics, with regard to both environment of airports and passengers. From the theoretical point of view, it is the final result of a whole strategy, involving physical understanding as a starting point and modeling as a tool for searching low-noise solutions. This is true for generating as well as propagating mechanisms. Within that scope, a complete simulation of the whole acoustic process from source to observer is not necessary. A partial model easy to run and able to predict the right trends is enough. The paper is devoted to a state-of-the-art in prediction methods. A presentation is given of both analytical and numerical techniques understood as complementary tools. Limitations of the former and expected future developments of the latter are discussed, together with hybrid techniques coupling computational fluid dynamics for the definition of sources and analytical acoustics. Examples are taken in the literature, not only in connection to turbofan engines, but also to other rotating blade technologies involving rotors and stators. The specificities of each application are used as a guideline for the best-suited approach.
\end{abstract}

9:40

3aNSf2. Predicting the effects of blade geometry on rotor-stator interaction noise. Nigel Peake (Dept. of Appl. Mathematics and Theoret. Phys., Silver St., Cambridge CB3 9EW, UK, n.peake@damtp.cam.ac.uk)

One of the principal noise generation mechanisms in modern high-bypass turbofan engines corresponds to the interaction between convected vortical disturbances from a rotor and a downstream stator row. In this talk, a method for predicting this noise will be described, which takes full account of the effects of the stator blade geometry. These effects include the distortion of the incident disturbances by the nonuniform flow around the stator, noise generation by flow sources close to the blade leading edges, and refraction as the noise propagates back upstream. The approach adopted involves the use of asymptotic analysis based on the realistic limits of large reduced frequency and small, but nonzero, blade camber and thickness. A new feature of this work concerns the behavior of the noise near modal cutoff. Previous ray-theoretic solutions diverge at these points, but a uniformly valid solution will be presented here which overcomes this difficulty. This makes it possible to use these techniques to predict the response of the stator to a whole turbulence spectrum, and not just to a single gust.

\section{Contributed Papers}

10:00

3aNSf3. Numerical analysis and active control of wake/blade-row interaction noise. C. J. Hwang and J. Y. Kuo (Inst. of Aeronautics and Astronautics, Natl. Cheng Kung Univ., Tainan, Taiwan, ROC, hwang@cfhp2.iaa.ncku.edu.tw)

A computational approach for investigating and controlling the wake/ blade-row interaction noise through the use of actuators or blowing/ suction on the stator blade surfaces is presented in this paper. This approach includes a solution-adaptive finite volume upwind scheme, adaptive/dynamic mesh technique, boundary treatments for aeroacoustic computations, and an active noise control algorithm. A two-dimensional section of a fan stage, which is composed of an upstream rotor and a downstream fan exit guide vane (FEGV), is investigated. By utilizing a rotor wake model, the acoustic fields of the FEGV are presented and analyzed to understand the accuracy and suitability of the present adaptive scheme. Also, the noise generation mechanics are studied. Finally, the active noise control algorithm is introduced. From the computational results, the reduction of noise due to actuators on the blade surfaces is more significant than that of using blowing/suction. The downstream propagating acoustic waves are more difficult to control than the upstream propagating waves. [Work supported by NSC.]

$$
\text { 10:20-10:40 Break }
$$

10:40

3aNSf4. An analytical model for upstream and downstream sound power from swept blade rows. Stewart A. L. Glegg (Ctr. for Acoust. and Vib., Florida Atlantic Univ., Boca Raton, FL 33431)

This paper will describe an analytical approach which can be used to calculate the upstream or downstream sound power generated by a swept blade row. The blade row is modeled using a linear cascade of flat plates with finite chord. The correct allowance for spanwise loading and gust 
convection is included. The value of this approach is that it can be used at very high reduced frequencies which are typically found in broadband fan noise applications. Results will be given which show the problems associated with only considering the noise sources at the leading edges of the blades and ignoring the trailing edges. The effects of blade sweep will also be illustrated by showing the radiated modal sound power as a function of blade sweep. [Work supported by NASA.]

\section{1:00}

3aNSf5. A cut-off attenuator with perforated plate in a centrifugal fan and its aeroacoustic experiments. Yadong Lu and Jing Tian (Inst. of Acoust., Chinese Acad. of Sci., P.O. Box 2712, Zhongguancun Rd., No. 17, Beijing 100080, PROC)

A new type of low-noise centrifugal fan with a special cut-off attenuator with a perforated plate has been invented and designed. The principle of noise suppression with this cut-off attenuator and its structure characteristics is introduced in this paper. A series of experimental comparisons of this type of centrifugal fan with a prototype centrifugal fan have been carried out. The experimental results show that the A-weighted noise level of a prototype fan without a cut-off attenuator with a perforated plate is 93 $\mathrm{dB}$. As a result of using this special attenuator, the noise level of a centrifugal fan is decreased to $88.3 \mathrm{~dB}$. Thus, sound attenuation due to this attenuator is $4.7 \mathrm{~dB}$. In addition, decreasing the airflow and keeping the rotating velocity of the fan unchangeable, noise reduction would be 3.5 to $5.0 \mathrm{~dB}$. It can be shown from the spectrum measurements that by using this cut-off attenuator with a perforated plate, not only the discrete frequency noise components of blade passing frequency and its harmonics, which predominate the sound level of a centrigual fan, can be attenuated, but also the broadband noise can be suppressed. Meanwhile, the aerodynamic performance of a centrifugal fan has not been greatly changed. [Work suported by NSFC.]
11:20

3aNSf6. Performance of acoustic liners for jet engine intake ducts. Matthew J. Greaves and Björn A. T. Petersson (Dept. of AAETS, Loughborough Univ., Loughborough LE11 3TU, England, M.J.Greaves@lboro.ac.uk)

In order to meet current and future noise emission regulations, it is necessary to reduce the noise generated by aircraft engines. One method is to line the duct with an attenuating configuration, and acoustic liners employing the Helmholtz resonator concept are now commonplace in jet engine intake ducts. Based on reports of core failure, the question of the mechanisms behind such failures, being the interaction of high sound pressure levels and shock waves with the liners, is addressed. Full-scale experimental pressure data are presented. Moreover, using the experimental data as input to a numerical model for nonrigid cavity walls, the induced stresses are analyzed. Results for typical liner configurations are given, and the nonlinear orifice effects considered. Possible alternative designs are examined and their advantages and disadvantages from an acoustic viewpoint discussed

\section{1:40}

3aNSf7. Acoustical forced oscillation diagnosis method of cracks for turbomachinery. Leonid M. Gelman and Lyudmila V. Kondratyuk (Dept. of Nondestructive Testing, Natl. Tech. Univ. of Ukraine, 37, Peremogy pr., Kiev, 252056, Ukraine)

A new acoustical forced oscillation diagnosis method of fatigue cracks for turbomachinery is proposed. The method uses nonstationary vibration excitation with variable frequency. Turbomachinery rotating details with fatigue cracks were considered as piecewise-linear systems. A new analytical approach and new analytical expressions of spectral density of narrow-band noise of rotating details are proposed. Experimental results of diagnostics of gas-turbine engine compressor blades based on noise emission are presented.

WEDNESDAY MORNING, 17 MARCH 1999

ROOM MA042, 7:55 A.M. TO 12:20 P.M.

Session 3aPAa

Physical Acoustics: Duct Acoustics I

Dirk Ronneberger, Cochair

Drittes Physikalisches Institut, University of Göttingen, Burgerstrasse 42-44, D-37073 Göttingen, Germany

Walter Eversman, Cochair

Mechanical and Aerospace Engineering and Engineering Mechanics, University of Missouri, 202 Mechanical Engineering, Rolla, Missouri 65401, USA

Chair's Introduction-7:55

Invited Papers

8:00

3aPAa1. Energy conservation, time-reversal invariance, and reciprocity. Willi Mohring (MPI fur Stromungsforschung, Box 2853, D-37018 Gottingen, Germany)

The scattering of sound waves at a connection of two different ducts is studied. They may carry a mean flow. The two ducts may differ geometrically or in the wall properties, and the transition from one duct to the other may be smooth or abrupt. The wall is assumed to react linearly, but not necessarily locally. An incident acoustic mode generates transmitted and reflected waves. For potential flow and lossless walls, an energy conservation theorem is valid. There is then also usually an invariance with respect to time reversal, which includes reversal of the mean flow. These properties imply reciprocity with reversed flow. Possible generalizations to vortical mean flow are discussed. These relations can be extended to a certain class of absorbing materials which are important in 
engineering and which show significant losses. This class consists of materials which are obtained from lossless materials by complexifying otherwise real material constants. (A resistance can be understood as an imaginary capacitance.) A reciprocity relation can then be obtained by analytic continuation, although there is neither energy conservation nor time-reversal invariance.

8:20

3aPAa2. Acoustic energy estimates in inhomogeneous moving media. F. Farassat (M.S. 461, NASA Langley Res. Ctr., Hampton, VA 23681) and Mark Farris (Midwestern State Univ., Wichita Falls, TX 76308)

In ducted fan engine noise research, there is a need for defining a simple and easy to use acoustic energy conservation law to help in quantification of noise-control techniques. There is a well-known conservation law relating acoustic energy and acoustic energy flux in the case of an isentropic irrotational flow. Several different approaches have been taken to generalize this conservation law. For example, Morfey [J. Sound Vib. (1971)] finds an identity by separating out the irrotational part of the perturbed flow. Myers [J. Fluid Mech. (1986)] is able to find a series of indentities by observing an algebraic relationship between the basic conservation of energy equation for a background flow and the underlying equations of motion. In an approximate sense, this algebraic relationship is preserved under perturbation. A third approach which seems to have not been pursued in the literature is a result known as Noether's theorem. There is a Lagrangian formulation for the Euler equation of fluid mechanics [M. E. Taylor, PDE's, Vol. 3, 1996]. Noether's theorem says that any group action that leaves the Lagrangian action invariant leads to a conserved quantity. This presentation will include a survey of current results regarding acoustic energy and preliminary results on the symmetries of the Lagrangian.

\section{8:40}

3aPAa3. A reverse flow theorem and acoustic reciprocity in compressible potential flows. Walter Eversman (Mech. and Aerosp. Eng. and Eng. Mech., Univ. of Missouri-Rolla, Rolla, MO 65401, eversman@umr.edu)

A reverse flow reciprocity theorem for acoustic propagation in compressible potential flows in nonuniform ducts is derived in terms of steady flow and acoustic velocity potentials. A similar result for a quasi-one-dimensional formulation is also presented. The theorem is specialized to investigate the characteristics of the acoustic modal scattering matrices for propagation in transition sections for axially symmetric ducts in the presence of high-speed flow. It is found that reflection coefficients for modes incident at the source plane in direct and reverse flow are related, and in particular, that diagonal elements in the direct and reverse flow reflection coefficient matrices are invariant. The same result is true for modes incident at the termination plane. The transmission coefficient matrix for modes incident at the source plane in direct flow is related to the transmission coefficient matrix for modes incident at the termination plane in reverse flow. Simpler but consistent results are demonstrated in the quasi-one-dimensional case where it is shown that the acoustic power transmission coefficient is invariant to the direction of flow. Numerical results from finite-element solutions are used to support the theoretical conclusions.

\section{9:00}

3aPAa4. Acoustics of ducted sources in motion. M. K. Myers (The George Washington Univ./JIAFS, MS 269, NASA Langley Res. Ctr., Hampton, VA 23681-2199, m.k.myers@larc.nasa.gov)

The radiation of sound into free space from sources enclosed in short ducts in subsonic forward motion has recently been of intense interest in connection with ducted fan aircraft engine noise technology. The current paper describes a boundary integral approach to calculating such fields which is applicable to arbitrary sources in circular ducts whose inner surfaces are lined with locally reacting absorbers. The theory is developed via a scattering formulation and leads to a system of two coupled singular boundary integral equations. For the case of a rigid inner duct surface, the system reduces to a single equation similar to that of unsteady subsonic lifting airfoil theory. The paper includes a description of the theoretical formulation and of a numerical solution of the governing integral equations. The numerical implementation is extremely economical to achieve, and its accuracy is affirmed by comparison with available results from alternate solutions obtained using CFD methods. Various results are presented to illustrate the effect of important parameters on the characteristics of the radiated sound.

\section{9:20}

3aPAa5. The effects of flanking transmission on sound attenuation in a finite-length lined duct, with mean flow. R. Kirby and A. Cummings (Faculty of Eng., Univ. of Hull, Hull HU6 7RX, UK, R.Kirby@eng.hull.ac.uk)

The acoustic performance of lined air-conditioning ducts is known to be compromised by the effects of flanking sound transmission. Two separate flanking paths have been identified; the first involves sound transmitted directly through the duct walls (sometimes termed radiation-by-pass) and the second involves the axial transmission of structural/acoustic waves along the duct walls. The attenuation of the internal sound field, particularly in relatively long ducts, is known to be limited by the effects of noise transmission caused by the formation of flanking paths. A model is described here which predicts the effect of both forms of flanking transmission on the attenuation of sound in a lined air-conditioning duct with mean flow in the central airway. Since most air-conditioning ductwork contains only a finite section of porous material, attenuation in both lined and unlined sections is included here. A finite-element eigenvalue solution is employed to couple the acoustic pressure field in the duct to the duct wall displacement. The principle of reciprocity is then used to model the effects of break-in. Finally, mode-matching techniques are employed to match the modal expansions across the discontinuity between the lined and unlined sections. [Financial support provided by the European Commission, BRITE/EURAM project FLODAC, Project No. BRPR CT97-0394.] 
3aPAa6. Sound transmission in a slowly varying flow duct with lossless liner. Sjoerd W. Rienstra (Dept. of Math. \& Computing Sci., Eindhoven Univ. of Technology, P.O. Box 513, 5600 MB Eindhoven, The Netherlands)

The explicit multiple-scales solution, presented in AIAA-No 98-2311, for modal sound transmission through slowly varying ducts with mean flow and acoustic lining, is extended to include the case of a (nominally) lossless liner $(Z=0)$. Especially the hydrodynamic or vortical mode is examined since this contains, in cooperation with higher order modes, the possibility of sound production or absorption by transfer of acoustic energy to and from the vorticity layer.

\section{0:00}

3aPAa7. Scattering at localized absorbers in a lined duct wall. Fridolin P. Mechel (Landhausstrasse 12, D-71120 Grafenau, Germany)

Homogeneous absorbing duct linings usually are tuned for medium and high-frequency attenuation. If they were tuned to low frequencies also, their thickness would become large. A possibility to add low-frequency attenuation to a broadband attenuation at higher frequencies consists in the combination of localized Helmholtz resonators with a broadband lining. The scattering cross section of the resonator orifice is large enough to permit some axial spacing between resonators. A method will be presented to show how the scattering at localized resonator orifices in a lined duct wall can be computed. The sound field in the duct is synthesized with silencer modes. The resonator orifice is introduced into the modal analysis as an effective volume flow source. Its strength is evaluated from the boundary conditions. Numerical examples show the wanted low-frequency attenuation maxima by arrangements with only a little increase of the silencer length and lining thickness.

\section{0:20-10:40 Break}

\section{Contributed Papers}

\section{0:40}

3aPAa8. Nonlinear standing waves in the two-tube structure. Ke Liu and Dah-You Maa (Inst. of Acoust., Academia Sinica, P.O. Box 2712, Beijing 100080, PROC)

Nonlinear standing waves have been studied in a structure consisting of two lengths of tubing with different diameters. Preliminary theory of this structure is proposed. The measurement system of experiment is presented. A 100-W loudspeaker driver is used as a sound source to excite the larger tube of $45-\mathrm{mm}$ diameter through a $10-\mathrm{mm}$ hole near one end, which is closed. The properties of the output at the end of the smaller tube, as well as their relation to the intput were investigated, and a resonance diagram of the two-tube structure is completed. The experimental results agree well with the predicted values. At the end of the smaller tube of 13-mm diameter, a sound pressure level (SPL) of $176.5 \mathrm{~dB}$ has been obtained with low distortion. Emphases are placed on the analysis of fundamental SPL. It is possible to drive the structure directly with a modern high power loudspeaker to produce high sound intensity in a more extended space. Further research is in progress. [Work supported by the National Science Foundation of China.]

\section{1:00}

3aPAa9. Acoustic pulse reflectometry for the measurement of tubular structures. Thomas J. MacGillivray and Clive A. Greated (Dept. of Phys. and Astron., The Kings Bldg., Mayfield Rd., Edinburgh EH9 3JZ, Scotland, tm@ph.ed.ac.uk)

Acoustic pulse reflectometry is used in the reconstruction of bore profile and the calculation of input impedance for tubular objects with varying cross section. The technique, previously employed in the measurement of musical wind instruments, enables differences of the order $0.1 \mathrm{~mm}$ in internal radii to be detected. Experimental measurements made on smallscale tubular objects of varying complexity demonstrates the effectiveness of reflectometry for profiling such structures. Certain configurations for which the accuracy of the technique begins to break down are also apparent. Examination of the results allows the effective length and area of an object to be determined from the reconstructed profile. Input impedance curves evaluated for small radius stepped and single-branched tubes exhibit differences compared to the corresponding theoretical expressions. This requires consideration of the object dimensions and also of viscous and thermal effects. A procedure for detecting blockages within the structures is developed through examination of different object profiles. The reconstructed profile for a structure containing an obstruction appears altered in shape compared to the unblocked case. The size of the blockage and the axial position are not, however, distinguishable.

\section{1:20}

3aPAa10. Negative resistances in nonlinear lumped acoustic systems and IC-engine source data. Fredrik Albertson (MWL, KTH, Teknikringen 8, SE-100 44 Stockholm, Sweden)

Negative real parts of impedances can be found in IC-engine source characterization measurements. There is an obvious problem with the physical interpretation of negative resistances, and consequently there have been many questions about the validity of such results. This paper is an attempt to explain the origin of the mentioned negative acoustic resistances. To this end, a simple nonlinear lumped acoustic system, that is, a force-driven damped mass-spring system, is analyzed. Since this is a common case in acoustic measurements, linear analysis is performed in spite of the obvious nonlinear properties. It is shown that the degree of nonlinearity in the system is crucial to the values of the acoustic impedance. Furthermore, it is shown that it is possible to achieve negative resistances already in a simple lumped system. Finally, these results are also compared to simulated and measured IC-engine source impedances.

\section{1:40}

3aPAa11. Sound-speed determination in a fluid-filled elastic waveguide. James A. McConnell (Penn State Univ., Grad. Prog. in Acoust., P.O. Box 30, State College, PA 16804 and Acoustech Corp., State College, PA 16804, univibe@sabine.acs.psu.edu), Kevin J. Bastyr, and Gerald C. Lauchle (Penn State Univ., State College, PA 16804)

Theory and test methods are presented for determining the longitudinal sound speed inside a fluid-filled elastic waveguide. The waveguide is a vertically oriented open-ended column of water equipped with a moving coil driver. The elastic properties of the waveguide reduce the sound speed to the point where a passive anechoic termination can be used effectively. This facilitates a compact device that exhibits plane progressive waves for sensor calibration at low frequencies. Once the sound speed is determined, then the size of the device and usable bandwidth are known. The sound speed is determined through measurement of the longitudinal resonance frequencies associated with the free vibration case of a duct having ideal rigid and pressure-release boundary conditions. In practice, however, the moving coil driver presents an impedance boundary condition (e.g., not ideally rigid) that inadvertently couples the mechanical system of the 
driver to the acoustic system of the waveguide. To circumvent this issue, frequency response measurements are made between a neutrally buoyant velocity sensor suspended in the fluid and an accelerometer mounted to the active face of the driver. In effect, the measurement decouples the two systems and provides the standing wave resonance frequencies of the duct having ideal boundary conditions.

\section{2:00}

3aPAa12. Sound propagation in a conical duct with flow. Rudolf N. Starobinski (Lab. d'Acoustique, URA 6613 CNRS, Univ. du Maine, av. O. Messiaen, BP 535, 72085 Le Mans, Cedex 9, France, starob@univ-lemans.fr)

The variations of acoustical exergy and acoustical mass velocity are used for a description of three-dimensional sound propagation in quasi one-dimensional moving medium in a conical duct of a different crosssectional shape. Solution for the sound propagation in this system is represented by the solution for a matched slightly deformed conical waveguide without flow (reciprocity part of the propagation) corrected by nonreciprocity time delay that takes into account convection of the sound by flow. Equivalent circuit for a sound propagation in a conical duct is represented by two successive separate elements: reciprocity one, which describes a sound propagation in the matched waveguide, and a nonreciprocity part represented by the element of nonreciprocity delay. For the particular dependencies of the cross-sectional area against duct axis, the solution for propagating sound is found as two propagated noninteracted advancing and backward waves. The Green's functions and equivalent circuits for internal and external sound excitations are found as their decomposition by the eigenmodes of the matched waveguide.

\title{
Contributed Poster
}

This poster will be on display in the Poster Gallery from Thursday to Friday, 18-19 March. Author will be at the poster from 10:00 a.m. to 12:00 noon on Thursday, 18 March.

3aPAa13. A new acoustic conductor. Fathy Shenoda and Albeer Shenoda, Jr. (Dept. of Acoust., Natl. Inst. for Standards, Tersa St., El-Haram, El-Giza, Code 12211, P.O. Box 136 Giza, Egypt, abdo@tqc.nis.sci.eg)

A new acoustic conductor was designed and experimentally studied. This conductor consists of a perfectly rigid pipe of constant cross-section area and is treated at its side wall with a small gradually perforated area. This experimental study aimed to determine its behavior and to outline the design procedures for optimizing its performance. The parameters (reflection coefficient $r$, normalized acoustic resistance $R$, and reactance $X$, at the conductor inlet) characterize the matching performance of the conductor, and were measured for different configurations and at different con- ditions. The design parameters such as the length, the graduality factor with which the side-perforated area increases, the function with which the side-perforated area is changed and reaches a maximum width $D_{m}$ at the end of the conductor, as well as the conductor cross-diameter, were separately studied in detail at different conditions. The conductor design is optimized at the conditions: The conductor terminates at a point where almost all the sound energy has been released. Within the effective frequency range, $r, R$, and $X$ have the following values: $r=0.1, R=1$, and $X=0$. The extensive experimental study and the great number of measurements at different conditions lead to an empirical formula for the calculation of cut-off frequency $f_{c}$ and optimum $D_{m}$.

WEDNESDAY MORNING, 17 MARCH 1999

ROOM MA005, 8:00 TO 11:20 A.M.

\section{Session 3aPAb}

\section{Physical Acoustics: Nonlinear Motion of Bubbles and Drops II}

\author{
R. Glynn Holt, Cochair \\ Department of Aerospace and Mechanical Engineering, Boston University, Boston, \\ Massachusetts 02215, USA \\ Joachim Holzfuss, Cochair \\ Institut für Angewandte Physik, TU Darmstadt, Schlossgartenstrasse 7, D-64289 Darmstadt, Germany
}

\section{Invited Papers}

\section{8:00}

3aPAb1. The Rayleigh-like collapse of a conical bubble. Tim G. Leighton and Ben T. Cox (Inst. of Sound and Vib. Res., Univ. of Southampton, Southampton SO17 1BJ, UK, tgl@isvr.soton.ac.uk)

This paper describes an experimental investigation of the collapse of a conical bubble, with measurement of the liquid and bubble gas pressures, and sonoluminescence, generated during the collapse. A theoretical analysis, based on the 1917 formulation of Rayleigh, adequately predicts the pressures, and the time scales as measured by high-speed photography, within the limitations of both theory and measurement. One implication of this novel apparatus lies in the ability to control the inertia associated with the liquid without changing its other properties. The inertia is key to the dynamics of the type of bubble collapse which is associated with sonoluminescence and the emission of strong rebound pressures into the liquid. Another implication of this study is the ability to image the collapsing meniscus and the gas and, to a certain extent, place sensors within the gas of the collapsing bubble. 
3aPAb2. Probing and stabilization of interfaces with acoustic radiation pressure: Application to bubbles and capillary bridges. David B. Thiessen, Mark J. Marr-Lyon, Philip L. Marston (Dept. of Phys., Washington State Univ., Pullman, WA 99164-2814), Thomas J. Asaki (Los Alamos Natl. Lab., Los Alamos, NM 87545), and Scot F. Morse (Naval Res. Lab., Washington, DC 20375)

Acoustic radiation pressure at interfaces can be used as a probe of dynamics and as a method of suppressing instability. Examples include the probing of the interfacial rheology of acoustically levitated bubbles in water in the presence of surfactants which are either insoluble [T. J. Asaki, D. B. Thiessen, and P. L. Marston, Phys. Rev. Lett. 75, 2686-2689 and 4336 (1995)] or soluble [T. J. Asaki and P. L. Marston, J. Acoust. Soc. Am. 102, 3372-3377 (1997)] in water. Modulated radiation pressure was also used to probe the dynamics of liquid bridges of oil surrounded by water [S. F. Morse, D. B. Thiessen, and P. L. Marston, Phys. Fluids 8, 3-5 (1996)] and to suppress the breakup of cylindrical bridges having lengths significantly beyond the natural Rayleigh-Plateau limiting length [M. J. Marr-Lyon, D. B. Thiessen, and P. L. Marston, J. Fluid Mech. 351, 345-357 (1997)]. In all of these examples the interfacial tension is significant for the normal modes of interest. Normal-mode frequencies can be significantly modified by using optical information for feedback control of the spatial distribution of the radiation pressure. [Work supported by the Office of Naval Research and by NASA.]

8:40

3aPAb3. Acoustic radiation forces on bubbles in real fluids. Alexander A. Doinikov (Inst. of Nuclear Problems, Byelorussian St. Univ., 11 Bobruiskaya St., Minsk 220050, Belarus, doinikov@inp.minsk.by)

Early theoretical studies on the acoustic radiation forces on gas bubbles have treated the surrounding medium as an ideal fluid devoid of viscosity and heat conduction, and for bubble-bubble interactions in addition with no compressibility. However, real fluids have all the above-mentioned properties and this fact may be very essential to the radiation forces. A more rigorous theory, allowing for the nonideality of the surrounding fluid, predicts a host of new exciting effects, such as the change of the sign of the force in a plane-traveling wave at high dissipation, long-range terms in the interbubble force which can cause the two bubbles to form a bound pair with a stable separation distance, etc. The purpose of this paper is to discuss such predictions.

\section{Contributed Papers}

\section{9:00}

3aPAb4. Cavitation bubbles' motions under ultrasound. Jean-Luc Laborde (EDF, Les Renardires ADEI, Rte. de Sens 77818, Moret-sur-Loing, Cedex, France, jean-luc.laborde@edfgdf.fr)

Propagation of power ultrasound (from 20 to $800 \mathrm{kHz}$ ) through a liquid initiates acoustic cavitation. Inceptions and germs grow into bubbles which collapse, possibly giving rise to extreme conditions of temperature and pressure (assessed to be up to $10000 \mathrm{~K}$ and $500 \mathrm{~atm}$ ). For instance, these conditions greatly enhance chemical reactions. A high-speed film shot at 500 fps clearly identifies stable and transient cavitation and shows bubble population phenomena. Clouds of bubbles grow up to ten times their emergence size during $15 \mathrm{~ms}$, and move at velocity around $50 \mathrm{~cm} / \mathrm{s}$. Mathematical modeling is performed as a new approach to predict where active bubbles are and how intense cavitation is. A calculation based on fluid dynamics equations is undertaken, as CFD codes have shown great interest because they provide not only the pressure field but also velocity and temperature fields. Two phase flows (liquid-bubbles) could be modeled. Nonlinearities arising from the dissipation of the acoustic wave are computed and lead to the calculation of the acoustic streaming. This result gives a better knowledge of bubbles' behavior. The comparison with photographs, a high-speed film, and particle image velocity show good agreement with simulations.

\section{9:20}

3aPAb5. Interaction of driven delay-coupled bubbles. Stefan Luther (Drittes Physikalisches Institut, Univ. Göttingen, Bürgerstrasse 42-44, D-37073 Göttingen, Germany, luther@physik3.gwdg.de) and John S. Allen (Univ. of Virginia, Charlottesville, VA 22902)

A rigorous derivation of the governing equation of two spherical, coupled, compressible bubbles indicates a variable time delay coupling term [Fujikawa and Takahira, Acustica 61, 188-199 (1986)]. However, the effects of this time delay were examined solely for free oscillations in that work. For acoustically driven bubbles, these time delay effects have been neglected in subsequent studies. This may be in part due to the computational complications involved in solving variable delay differential equations. In this study, delay effects are rigorously explored for acoustically forced bubbles. An equation for the overall energy balance of the system is derived and used to investigate novel Bjerknes Force effects. A direct comparison with nondelay formulations reveals important differences for certain parameter regimes. [Work supported by "Graduiertenkolleg Strömungsinstabilität und Turbulenz.']

\section{9:40-10:00 Break}

\section{0:00}

3aPAb6. Control of nonlinear bubble dynamics. Andrew J. Szeri and Matthew A. Wyczalkowski (Univ. of California, Dept. of Mech. Eng., Berkeley, CA 94720-1740, aszeri@me.berkeley.edu)

Bubbles, either free or encapsulated, are valuable as contrast agents in diagnostic ultrasound in medicine. In this capacity, the compressibility of microbubbles is exploited to increase the acoustic backscatter from internal vessels and cavities of interest after injection into the patient. The scattering cross section of the contrast agent is further increased by driving resonant radial pulsations of the microbubbles with the ultrasound field. Recent derivatives of this basic technique rely on nonlinear aspects of the bubble response to reduce clutter and thereby to extend imaging capability to new areas. Unlike the surrounding tissue, these nonlinear bubble oscillations have a backscatter signature at a different frequency than the forcing, and so are especially useful in distinguishing the area to be imaged. New control capabilities are described for changing the architecture of the bubble dynamics phase portrait and response diagram so as to enhance their utility in imaging applications. [Work supported by NSF.]

\section{0:20}

3aPAb7. Effects of shape oscillations on bubble dynamics and coalescence. Zaichun Feng (Dept. of Mech. Eng., MIT, Cambridge, MA 02139)

When a bubble is excited by an acoustic field, in addition to the volume oscillation, the bubble may deviate from the spherical shape and undergo shape oscillations. The shape oscillations can be directly caused by the anisotropic pressure or they can be caused by the energy transfer 
from the volume mode through nonlinear modal couplings. The shape oscillations influence the bubble dynamics in many ways. First of all, shape oscillations can limit the volume responses of the bubble since the onset of shape oscillations can lead to the development of localized large curvature and hence the breakup of the bubble. Second, the resonance coupling between the volume mode and a shape mode can give rise to the occurrence of chaos even when the acoustic forcings are so weak that the bubble oscillates close to its equilibrium radius. Third, the coupling between two or more shape oscillation modes can lead to translational motions of the bubble centroid; this can indirectly affect the bubble coalescence in an acoustic field. Finally, the shape oscillations may influence the coalescence through modifying the short-range attractive forces between two neighboring bubbles.

\section{0:40}

3aPAb8. Bubble response to the amplitude modulation signal near the threshold of dynamical stability. Alexey O. Maksimov and Ekaterina V. Sosedko (Pacific Oceanological Inst., 43 Baltic St., Vladivostok, 690041, Russia, root\%dan86@poi.marine.su)

There is a range of acoustic techniques for characterizing bubble populations within liquids [Leighton et al., Ultrasonics 34, 661-667 (1996)]. The linear resonant backscattering was the first detection technique. The great difference between the compressibility of gas in the bubble and surrounding liquid leads to the easy manifestation of nonlinear effects, the simplest of which is the presence of harmonics at $2 \omega_{p}, 3 \omega_{p}$, etc., of the pure tone driving frequency $\omega_{p}$ in the scattering signal. However, bubble pulsation of finite amplitude results not only in the harmonics generation. The nonlinear resonance characterized by multistable oscillation states arising from the saddle-node bifurcations is a significant example. The emphasis of this study is to propose a technique for bubble sizing by using the ability of a bubble as a nonlinear oscillatory system to amplify weak signals near the threshold of dynamical stability. The analytical as well as numerical solutions have been derived for the nonlinear bubble response to the modulation driving pressure. The magnitude and the form of the acoustical signal reradiated near the threshold of dynamical stability are drastically different from the modulation of incident wave and enable the characterization of the bubble population in liquid.

\section{1:00}

3aPAb9. An acoustic levitation technique for measuring the rheology of foam near the order-disorder transition. R. Glynn Holt, Cory C. Clarke, and J. Gregory McDaniel (Dept. of Aerosp. and Mech. Eng., Boston Univ., 110 Cummington St., Boston, MA 02215, rgholt@bu.edu)

Understanding the rheological behavior of foams is important as a basic problem in fluid physics, and as a practical problem in many industries. Foams are tremendously important in a variety of applications. The most important quality of a foam in many of these applications is its response to imposed strain, or its rheological behavior. Yet there exists almost no experimental data on the rheological properties of real 3-D foams. This is due in large part to the earth-based requirements for contact containment, and to the fact that gravity-induced drainage quickly destroys all but the "driest" foams. A unique method to provide noncontact control and manipulation of foam samples, via acoustic levitation, is described. This levitation technique, coupled with low-gravity conditions, will enable investigation of dry (ordered) and wet (disordered) foams. The analysis which will allow determination of a foam's yield stress, effective bulk modulus, and effective bulk viscosity from the observed response of a foam sample to quasistatic, step function, and periodic acoustic and ambient pressure changes is outlined. Experimental results will be presented for foams experiencing small deformations. The results will be analyzed to determine if the foam may be modeled as a viscoelastic solid. [Work supported by NASA.]

WEDNESDAY MORNING, 17 MARCH 1999

ROOM MA041, 8:00 A.M. TO 12:20 P.M.

\title{
Session 3aPAc
}

\section{Physical Acoustics: Periodic and Layered Media}

\author{
Robert M. Keolian, Cochair \\ Physics Department, Naval Postgraduate School, Monterey, California 93943, USA \\ Antoni Sliwinski, Cochair \\ Institute of Experimental Physics, University of Gdansk, Wita Stwosza 57, 80-952 Gdansk, Poland
}

Contributed Papers

8:00

\begin{abstract}
3aPAc1. Time-domain Floquet-wave Green's function for an infinite sequentially excited periodic line array of point radiators. Leopold B. Felsen (Dept. of Aerosp. and Mech. Eng., Boston Univ., 110 Cummington St., Boston, MA 02115, lbf@enga.bu.edu) and Filippo Capolino (Dept. of Info. Eng., Univ. of Siena, Italy)
\end{abstract}

A variety of new synthetic materials, especially those exploiting sonic or photonic bandgaps, are based on a background with strict or perturbed periodicity, which may be subjected to rapidly switched external modulation. This has motivated the present prototype study of the time domain
Green's function for an infinite sequentially excited periodic line array of point radiators or scatterers. Such Green's functions, which parameterize TD Floquet wave (FW) dispersion, are the analytic building blocks for finite planar arrays with physical radiators. Various techniques are employed to develop exact expressions for the dispersive TDFW's synthesized by Poisson summation over the linearly time-delayed nondispersive impulsive point source radiators. These techniques include Fourier inversion of frequency domain FW's, TD spectral wavenumber decomposition and recombination, and use of complex space-time analytic signals. Each formulation furnishes different insights into propagating as well as evanescent TDFW spectra, and into TDFW dispersion. Some basic questions concerning causality and TDFW "physical observability" are posed and answered. 
ing of components which can be designated a physical interpretation. These components, i.e., partial pressures related to corresponding geom-

3aPAc2. Propagation features of evanescent acoustic waves. Adriano Alippi, Andrea Bettucci, Francis A. Farrelly, and Massimo Germano (Dept. of Energetics, Univ. of Rome “'La Sapienza,'” via Scarpa 14, 00161 Rome, Italy, alippi@uniroma1.it)

Evanescent waves are not true propagating waves, so questions regarding their traversal time or propagation direction are ill posed. However, physical phenomena related to the time delay of pulsed evanescent perturbations, or refraction effects through a discontinuity, can be easily put into evidence in acoustics by proper choice of acoustic modes and geometrical configuration. In this paper, lateral displacement and time delay through forbidden barriers of Lamb mode waves with contradirected phase and group velocities are studied. Low noise detection achieved by cutting off spurious mode generation and a common objection made toward experiments of this type is avoided. This objection states that the highest frequency components of the pulse travel faster and are less attenuated than the main carrier frequency of the packet, distorting the original pulse to such an extent as to render measurements unrelated to barrier traversal times. Elastic waves generated with piezoelectric transducers are injected into aluminum plates through wedge-shaped Lucite waveguides so as to generate the appropriate Lamb mode which traverses a forbidden region and is picked up after it with a similar setup of transducer and waveguide. Various configurations of wave barriers have been measured and possible theoretical interpretation is given.

\section{8:40}

3aPAc3. Experimental analysis of the propagation of elastic waves in a chain of spherical beads. M. de Billy and X. Jia (Groupe de Physique des Solides, Universités Paris 6 et 7, 2, place Jussieu, 75251 Paris Cedex 05, France, debilly@gps.jussieu.fr)

Granular materials are widely encountered in many domains such as soil mechanics, geophysics, and physics of condensed matter. Consequently, these media have been extensively analyzed in recent years [A. N. Bogdanov and A. T. Skvortsov, Sov. Phys. Acoust. 38, 224-226 (1992) and C. Coste et al., Phys. Rev. E 56, 6104-6117 (1997)]. The present experimental study is concerned with the propagation of an elastic wave through a chain of spherical beads submitted to a static force. Two piezoelectric transducers are used for the generation and the detection of the trainwaves. A low-frequency signal is observed in the transmitted acoustic waves along the chain of beads. The central frequency $(25<f$ $<200 \mathrm{kHz}$ ) of this signal varies with the static force exerted on the beads and also with the diameter $(d)$ of the spherical particles $(2<d$ $<8 \mathrm{~mm}$ ). For a constant value of the static force, the amplitude of the signal is strongly affected by the material of the solid medium which support the acoustic wave (brass, steel, nylon). The increasing of the sound velocity with the applied force seems to verify the prediction based on the Hertzian contact.

\section{9:00}

3aPAc4. A closed formulation of sound fields in front of periodical absorbents. Dag Holmberg and Erling Nilsson (Dept. of Eng. Acoust., LTH, Lund Univ., P.O. Box 118, SE221 00 Lund, Sweden, Dag.Holmberg@kstr.lth.se)

Periodic resonant absorbers are known to give excessive back scattering for frequencies above a limit which is governed by the geometric proportions of the periodicity. One way to model this phenomenon is to transform the geometry into the wave number domain and solve the corresponding equation with respect to the pressure field. A drawback is that the equation matrix consists of an infinite (but relatively fast converging) set of harmonic components and therefore has to be truncated in order to be solved. However, it is possible to make use of the Poisson sum formula and obtain a closed formulation which can be solved in the wave domain without truncation. Furthermore, it is possible to transform the exact solution back to the spatial domain. This will yield an infinite series consistetry, which constitute an exact solution, can then be truncated in a controlled and physical way.

\section{9:20}

3aPAc5. Sonic band structure and surface localized modes in a density-modulated system: An experimental and theoretical study. Christopher Carr (Dept. of Phys., Univ. of Davis, Davis, CA) and Roger Yu (Central Washington Univ., Ellensburg, WA 98926)

As a result of the Bloch theory, the eigenstates of an electron in a strictly periodic potential are extended and the eigenenergy levels form allowed energy bands separated by forbidden gaps. In the study reported here, a finite air-filled tube with arbitrary mass density modulation is considered which allows the inclusion of the effect of disorder. Since this theoretical model is finite, it was possible to make direct comparisons with the experiment. The baffled tube is excited with the sound source emitted at one end. The source can deliver short pulses as well as harmonic oscillations. Both normal modes analysis (NMA) and pulse analysis (PA) were used. The spectra obtained from both methods are compared with the theoretical prediction. For the tube with evenly spaced baffles, three passing frequency bands were found. More interestingly, a few "surface", localized resonant modes whose frequencies lie in the gaps were discovered. The wave functions of the surface modes are confined within a few sections of divided tube at both ends. It was found that the acoustic intensity of higher frequency bands is drastically lower than that of the lowest band, which suggests that the baffled tube can be used as a frequency filter.

9:40

3aPAc6. Multilayered Rayleigh waves in anisotropic periodically multilayered media: Comparison between theory and experiments using the acousto-optic technique. Catherine Potel, Jean-Francois de Belleval (Univ. Technol. Compiegne (UTC), LG2mS, UPRES ass. CNRS, BP 20529, 60205 Compiegne, France), Stefaan Devolder, Martine Wevers (Katholieke Univ. Leuven, Leuven, Belgium), and Oswald Leroy (Katholieke Univ. Leuven, Kortrijk, Belgium)

The propagation of surface waves, particularly Rayleigh waves, has become the subject of a large number of studies. As far as stratified media are concerned, many of the studies deal with an infinite substrate medium below the stratified or nonstratified medium, but, as far as infinite periodically stratified media are concerned, there is no complete work on leaky waves that behave as Rayleigh waves. By analogy with the behavior of the Rayleigh wave in isotropic media, the properties of what this group termed in previous papers "multilayered Rayleigh wave" in infinite anisotropic periodically multilayered media, were studied. These waves are a linear combination of three inhomogeneous Floquet waves and are dispersive. This theory was developed for plane waves. This paper aims to validate this theory by using an experimental acousto-optic technique linked to bounded beams. The experiments were carried out on a composite of carbon/epoxy layers. The coincidence of experimental and calculated dispersion curves of the multilayered Rayleigh modes was found to be quite good. [Work partially supported by the National Fund for Scientific Research and the Flemisch Institute for the Encouragement of the Scientific and Technological Research in Industry. Aerospatiale (France) has provided the characterized sample of carbon/epoxy composite.]

$$
\text { 10:00-10:20 Break }
$$

10:20

3aPAc7. Modal waves in periodically multilayered fluid structures. Philippe Gatignol, Catherine Potel, and Jean-Francois de Belleval (Univ. Techn. Compiegne (UTC), LG2mS, UPRES ass. CNRS, BP 20529, 60205 Compiegne, France)

This paper aims to study the existence of modal waves and in particular of surface waves, in periodically multilayered fluid media. Generally speaking, these waves are propagation modes for which the acoustical energy propagates along the layers, whereas it remains bounded in a di- 
rection perpendicular to the layers. Periodically multilayered media are here studied by the transfer matrix method. The calculus of eigenvalues of the transfer matrix for one period permits to distinguish between passing and stopping regions in the wave number/frequency plane. The transfer matrix for a finite number of periods can be calculated by using Tchebychef polynomials. The writing of the boundary conditions for a finite structure surrounded by vacuum leads to a factorized equation. Thus, two families of guided waves are obtained: the first one corresponds to structure modes which exist in passing regions only. The second one only depends on the period properties and corresponds to inhomogeneous Floquet waves. When the periodic structure is infinite, the modes of this second family coincide with surface waves.

\section{0:40}

3aPAc8. A theoretical investigation of sound transmission through a layered periodic structure in water. Aly H. A. Moustafa (Faculty of Electron. Eng., Dept. of Commun., Menoufla Univ., Menouf-32952, Egypt)

This paper presents a theoretical study of sonic passbands and stopbands that occur with a periodic one-dimensional structure of plates in water. The study uses the characteristic matrix method and considers a periodic structure of some materials that are characterized by a constant density and phase speed: perspex, aluminum, and steel. The computed results show the variation of the pressure transmission coefficient with frequency for various systems of plate configurations in water, and takes into account the attenuation of the plate material. The pattern of the results indicates clearly that sound propagation can be prohibited at certain frequencies while free propagation is allowed at others. The results compare very well with published data that are obtained using other methods.

\section{1:00}

3aPAc9. Modeling of inhomogeneous monochromatic plane wave at the interface between two arbitrary homogeneous anisotropic absorbent media. Bruno Roge, Patrick Lanceleur, and Jean-Francois de Belleval (UTC-LG2mS (UPRES A CNRS 6066), Centre de Recherches de Royallieu, BP 20.529, 60205 Compiegne Cedex, France)

This work aims to model the interaction of an inhomogeneous monochromatic oblique plane wave with a plane interface separating two semiinfinite homogeneous media. These media can be either isotropic or anisotropic, absorbent or not. A numerical method is proposed to generalize previously developed procedures, which were restricted to the case of incident homogeneous waves, interacting with absorbing materials. In this case the waves propagating are always inhomogeneous. Due to this inhomogeneity of the waves, the choice of compatible parameter for the incident wave is to be done on an energy criterion and not necessarily on a radiation one. The computation of all the parameters of all the involved waves makes several unexpected phenomena or behaviors to arise. For instance, by applying the study to the case of a water/nickel monocrystal interface, incident directions for which the energy flow vector is directed toward the transmission medium, whereas the slowness vector is directed toward the incident medium, are observed. The appearance of leaps in the reflection coefficient will then be explained by an orientation change of the slowness vector, which results from a forward/return of the energy flow vector through the interface.
11:20

3aPAc10. Micellar structures in layered oil/water surface systems studied with photoacoustics spectroscopy. Stanislaw J. Pogorzelski, Janusz Szurkowski, and Antoni Sliwinski (Inst. of Exp. Phys., Univ. of Gdansk, Wita Stwosza 57, 80-952, Gdansk, Poland)

The model layered system consisting of thin olive oil layers (ranging from 25 to $250 \mu \mathrm{m}$ in thickness) spread on the water surface was studied using a photoacoustic method. The classical Rosenzweig-Gersho model assuming a one-dimensional heat flow in the sample was adopted as a depth profiling approach to interpret data in terms of the thermal diffusion length and thermal diffusivity. Distinctive changes in the PA signal phase and its amplitude discovered at the air/oil and oil/water interfaces, as well as at the depth of 9-13 $\mu \mathrm{m}$ beneath the oil surface, point out the possibility of formation of organized adsorbed interfacial layers and micellar structures in a bulk oil phase of surfactants, if present in an oil medium at a certain concentration (above critical micellar concentration CMC) and temperature (Kraft point). Such organized structures have different thermodynamic parameters like specific heat or thermal conductivity that affect to a great extent the photoacoustic effect used in the depth profiling sensing.

\section{1:40}

3aPAc11. Time-of-flight measurements on high-frequency surface acoustic phonons in silicon. D. M. Photiadis (Naval Res. Lab., Washington, DC) and J. Ding (ThermaWave, Inc., San Francisco, CA)

Results from time-of-flight measurements of high-frequency surface acoustic phonons $(50-500 \mathrm{GHz})$ in (001) silicon wafers at low temperatures are reported. Pulsed laser excitation is used for the generation of the high-frequency surface acoustic phonons and aluminum edge bolometers are used as detectors. The times defined by the fast initial response of the edge bolometer correspond to surface acoustic delays in silicon. Late time responses consistant with echoes from the back face of the wafer were also observed. These results and the accompanying analysis will be discussed.

\section{2:00}

3aPAc12. Surface acoustic waves in proton-exchanged lithium niobate and lithium tantalate. Daumantas Ciplys and Romualdas Rimeika (Vilnius Univ., Faculty of Phys., Lab. of Physical Acoust., Sauletekio 9, Vilnius 2054, Lithuania)

The use of surface acoustic waves in integrated optical devices seems to be very promising for applications in modern telecommunication and data processing technologies. The studies of surface acoustic wave propagation in lithium niobate and lithium tantalate with optical waveguides formed by the proton exchange technique are reported here. The influence of protonated layer parameters on the SAW propagation characteristics is investigated for various crystal cuts and waveguide fabrication conditions. The acoustic velocity and attenuation have been studied by the laser probe method. The effective electromechanical coupling coefficient of the protonated crystal surface has been measured by the method of thin metal film evaporation. Possibilities for controlling both the optical and acoustic properties of proton-exchanged waveguides by the post-exchange annealing have been studied. The results of experimental studies of SAW propagation parameters are compared with those of theoretical calculations performed for a layered structure, and the parameters of a protonated layer are evaluated. The light modulator based on the interaction of surface acoustic wave and guided optical modes is demonstrated. 


\title{
Session 3aPPa
}

\section{Psychological and Physiological Acoustics and Speech Communication: Localization and Speech Perception in Realistic Acoustic Environments}

\author{
Ruth Y. Litovsky, Cochair \\ Department of Biomedical Engineering, Boston University, 44 Cummington Street, Boston, Massachusetts 02215, USA \\ Tammo Houtgast, Cochair \\ TNO Human Factors Research Institute, Kampweg 5, 3769 DE Soesterberg, The Netherlands \\ H. Steven Colburn, Cochair \\ Department of Biomedical Engineering, Boston University, 110 Cummington Street, Boston, Massachusetts 02215, USA \\ Chair's Introduction-7:55 \\ Invited Papers
}

8:00

3aPPa1. Localization of sound in reverberant spaces. William M. Hartmann (Phys. and Astron., Michigan State Univ., East Lansing, MI 48824) and Brad Rakerd (Michigan State Univ., East Lansing, MI 48824)

Sound sources in the horizontal plane can be localized on the basis of interaural time differences (ITD) or interaural level differences (ILD). It will be argued that without the benefit of an onset transient, listeners can employ only the ILD to localize steady-state broadband sources in a typical reverberant environment. The ITD provides a useful cue only if the cross-correlation (CC) function is adequately large. At low frequencies, a CC peak near 0.1 is barely adequate, but in a reverberant room the physical CC peaks are often less than that. The physical CC peak increases with increasing noise band frequency, but the value of the CC peak that is required for localization increases faster. Therefore, ITD is not useful at any frequency. By contrast, a comparison of physical and psychoacoustical measurements shows that ILD is a useful cue. An optimized ILD model is able to account for human sound localization performance in a reverberant space for broadband and low-pass filtered noises, for both normal listeners and listeners with a high-frequency hearing loss. [Work supported by the NIDCD, Grant DC00181.]

\section{8:20}

3aPPa2. Localization of distracted speech and noise in reverberant and anechoic environments. Klaus Hartung and Jonas Braasch (Inst. of Commun. Acoust., Ruhr Univ., D-44780 Bochum, Germany, hartung@ika.ruhr-uni-bochum.de)

Eleven listeners were instructed to localize a test signal in the presence of a distractor sound in an anechoic environment and in a reverberant environment. The experiment was conducted in an auditory virtual environment using individually measured headrelated transfer functions. A rectangular room $(6 \times 5 \times 3 \mathrm{~m})$ was created using the mirror-image method to simulate the reverberant environment. The distractor (noise burst, 500-ms duration, $20-\mathrm{ms} \cos ^{2}$-ramp) was placed at 0,30 , or $90^{\circ}$ azimuth. The signals, random noise bursts (200-ms duration, 20-ms $\cos ^{2}$-ramp) or the German spoken word "zuende" were presented from 13 directions in the frontal hemisphere of the horizontal plane at different signal-to-noise ratios $(\mathrm{S} / \mathrm{N})$. For some $\mathrm{S} / \mathrm{N}$, the listeners were unable to discriminate between a number of directions of incidence of the test signal (median test). The distinguishability of locations (DOL) was introduced to measure the percentage of discriminated locations. The DOL is a function of the $\mathrm{S} / \mathrm{N}$, decreasing rapidly below a threshold of $-12 \mathrm{~dB}$ in an anechoic environment. This threshold increased by about $5 \mathrm{~dB}$ under reverberant conditions. The reduced DOL was confirmed by a 2A-4IFC experiment. If a distractor were present in the anechoic environment, the perceived directions of the test signal were shifted significantly in the direction opposite to the distractor location for all distractor directions (sign test, $\alpha$ $=3 D 0.01$. . [Work supported by DFG.]

\section{8:40}

3aPPa3. The precedence effect: Fusion and localization dominance. William A. Yost (Parmly Hearing Inst., Loyola Univ. of Chicago, 6525 N. Sheridan Rd., Chicago, IL 60626)

Fusion and localization dominance are two aspects of processing sounds in reverberant spaces. If a single sound source is perceived when a source and its reflections are presented then a perceptual fusion of the source and reflection has occurred. Localization dominance refers to the spatial location of a sound source and its reflections being dominated by the location of the source. Four listeners were presented a transient sound from one loudspeaker (source) in a sound-deadened room followed a few milliseconds later by the presentation of the transient at another loudspeaker (reflection). The listeners determined how many sound sources were detected (fusion) and the spatial location of these sources (localization dominance). The results will be discussed in terms of the temporal delay between the source and reflection $(0.5$ to $64 \mathrm{~ms})$, the number of presentations of the source and reflection $(1,3,6,7$, $9,12)$, and three conditions of switching the source and reflection after the sixth presentation: no switch, the location of the source and the reflection were reversed (reversal switch), and the location of the source and reflection were moved in the same direction (lateral switch). [Work suported by NIDCD and AFOSR.] 
3aPPa4. Physiological studies and neural mechanisms of echo suppression in the inferior colliculus of the cat. Ruth Y. Litovsky (Boston Univ., Dept. of Biomed. Eng., 44 Cummington St., Boston, MA 02215 and Eaton-Peabody Lab., Massachusetts Eye and Ear Infirmary), Bertrand Delgutte (Massachusetts Eye and Ear Infirmary, Boston, MA 02114), and Tom C. T. Yin (Univ. of Wisconsin, Madison, WI 53706)

Neurons in the inferior colliculus (IC) are sensitive to stimulus direction in free field and to individual directional cues, such as interaural disparities in time (ITD) and level (ILD). Our single-unit recordings in the IC of anesthetized cats explored neural mechanisms in the IC that might mediate echo suppression, using free-field and virtual space (VS) stimuli. Click pairs were used to simulate a sound source (lead) followed by a single reflection (lag) at delays of 1-100 ms. In both free field and VS, correlates of psychophysical echo suppression were found in the responses of most IC neurons. In many neurons, the suppression was directionally dependent in a way that could be linearly predicted from the response to the lead, with the most effective lead locations giving maximum suppression. Some neurons showed a decoupling between echo suppression and the directional response to the lead, suggesting that excitation and suppression are mediated by different neural mechanisms. Further decoupling was achieved by selectively holding constant some directional cues such as ITD and ILD in the VS stimuli. Results suggest that neural suppression in the IC depends on specific directional cues and is therefore a likely correlate of echo suppression in spatial hearing. [Work supported by NIH.]

\section{9:20}

3aPPa5. The cocktail party effect: Research and applications. Adelbert W. Bronkhorst (TNO Human Factors Res. Inst., P.O. Box 23, 3769 ZG Soesterberg, The Netherlands, bronkhorst@tm.tno.nl)

The "cocktail party problem," defined in 1953 by Cherry [J. Acoust. Soc. Am. 25, 975-979 (1953)] as "how do we recognize what one person is saying when others are speaking at the same time" has been the subject of a considerable number of studies in the last 45 years. Whereas Cherry already hypothesized that there are multiple effects that contribute to the ability to extract one speaker out of a babble, the term "cocktail party effect" has been claimed by only one of them: the influence of spatial separation of the voices. One important conclusion from past research on the cocktail party effect is that the truly binaural gain (the "unmasking") is, in general, relatively small $(2-4 \mathrm{~dB})$. The additional "acoustic" gain due to head shadow effects may be larger but depends strongly on the circumstances. In the present paper the research will be reviewed including recent evidence that much larger binaural gains may occur when the masker is "informational" in nature [Kidd et al., J. Acoust. Soc. Am. 104, 422-431 (1998)]. In addition, several applications based on the cocktail party effect, such as the use of a virtual auditory display for multiple-speaker communication, will be highlighted.

\section{9:40}

3aPPa6. Binaural hearing by listeners with hearing impairments. Monica L. Hawley, Ruth Y. Litovsky, and H. Steven Colburn (Hearing Res. Ctr. and Biomed. Eng., Boston Univ., 44 Cummington St., Boston, MA 02215, mlh@bu.edu)

The ability of listeners with hearing impairments to understand speech in the presence of competing speech sources is often compromised; however, the extent to which poor binaural hearing contributes to this compromise is not known. These studies are aimed at relating listeners' abilities to utilize binaural information in speech-based and non-speech-based tasks. In one set of measurements, speech intelligibility and localization of speech material were tested in the presence of one to three competing sources, placed at various spatial configurations. A second set of measurements tested the sensitivity to binaural information (i.e., MLD, ITD, and ILD discrimination). Listeners who obtained maximal binaural benefit in the speech intelligibility tasks generally showed good performance in all other tasks. Listeners who showed normal ITD/ILD discrimination could not always exploit that ability in more complicated tasks (MLD and speech-based tasks). Data were compared with the prediction of a model [Zurek, in Acoustical Factors Affecting Hearing Aid Performance (Allyn and Bacon, Boston, 1993)] which separates benefit of the "better monaural ear" and binaural interaction. Prediction was best when individuals MLDs were used to predict the binaural interaction term. Knowledge of the individual's binaural abilities may help clinicians choose an appropriate rehabilitative strategy. [Work supported by NIH.]

10:00-10:20 Break

10:20

3aPPa7. The "cocktail-party effect" viewed through the lens of psychophysics. Pierre L. Divenyi (Exp. Audiol. Res., V. A. Medical Ctr., Martinez, CA 94553)

In an attempt to understand the psychophysical processes underlying the "cocktail-party effect" - a phenomenon whereby one speech signal is extracted from the ensemble of other speech signals distributed in space-it has been reduced to the psychophysical task of segregating two concurrent signals $X_{1}(t)$ and $X_{2}(t)$ differing with respect to various attributes. These attributes represent three orthogonal "canonica" dimensions-spatial location, temporal (low-frequency envelope AM) structure, and pitch, although the pitch dimension is split into spectral and virtual pitch components. In any segregation experiment, the two signals differ along at least two attributes; segregation is assumed when each signal is associated with the correct values on each dimension. At segregation threshold, there is a trade-off over a certain range, where an increased difference along one dimension leads to a reduced difference along another. Along each dimension, there is also a "critical" difference at which the trade-off no longer exists. When converted to speech parameter coordinates, these critical differences-for azimuthal, spectral, fundamental frequency, and modulation frequency separation - can provide psychophysical predictions for conditions under which a speech signal could be successfully recovered in a real-life cocktail-party situation. [Work supported by the National Institute on Aging and the VA.] 
3aPPa8. Application of localization models to noise suppression in hearing aids. Volker Hohmann, Stephan Albani, and Johannes Nix (AG Medizinische Physik, Univ. of Oldenburg, Oldenburg, Germany)

One major problem of hearing-impaired people is the cocktail-party effect, i.e., the reduced ability to understand speech in noisy environments. Normal-hearing listeners use binaural information, i.e., interaural level and phase/time differences, in addition to monaural cues to localize sound sources in the environment. Based on the localization result and on the monaural cues, the listener performs a significant noise reduction by concentrating on one of the sources. Noise reduction algorithms have been proposed that try to imitate the binaural information processing in the hearing system by using two microphones at both ears and a central processor. This setup allows for the use of interaural parameters to estimate the direction of sound sources and to attenuate unwanted sound source directions. However, in noisy environments the binaural parameters become noisy as well, reducing the accuracy of the sound source estimation and therefore reducing the maximum performance of the noise reduction algorithm. In this presentation, a localization algorithm is introduced, which uses a statistical description of the binaural parameters in order to perform a robust estimation of sound source directions in noisy environments. Noise reduction schemes based on this localization algorithm are discussed.

\section{Contributed Papers}

11:00

3aPPa9. Speech perception in telephone situations under ambient noise conditions. Thomas Hempel, Sebastian Möller, Ute Jekosch (Inst. of Commun. Acoust., Ruhr Univ. Bochum, D-44780 Bochum, Germany, hempel@ika.ruhr-uni-bochum.de), Sabine Meunier, and Xavier Regal (Lab. de Mécanique et d'Acoust., CNRS, F-13402 Marseille, France)

In a telephone situation, the partners of a conversation generally experience ambient noise because they are located in acoustically realistic environments. The impact of the ambient noise on the quality of a telecommunication service is a double one: on the one hand, the noise deteriorates the acoustical speech signal either directly or via the sidetone path of a telephone handset, thus leading to lower intelligibility and overall quality. On the other hand, it distracts the listener's attention. Models which try to predict the effect of ambient noise for telephone network planning purposes in general only regard a diffuse room noise level. However, localization of possible ambient noise sources can help the listener to concentrate on the conversation rather than being distracted by the noise. A conversation test has been carried out in order to investigate the effect of ambient noise in both handset and hands-free telephone situations. Noise was either diffuse or localized at different positions relative to the listener. The test results are compared to intelligibility measures for masked speech given in literature. The investigation can help to quantify the impact of localized ambient noise and gives information to design realistic ambient noise environments for telephone or teleconferencing scenarios.

\section{1:20}

3aPPa10. Effects of aging on sound localization and speech understanding in realistic listening situations. Janet Koehnke and Joan Besing (U.S. Army Res. Lab., Human Res. and Eng. Directorate Bldg. 520, APG, MD, jkoehnke@arl.mil)

The ability to localize sound in quiet and noise and understand speech in noise was measured for four groups of subjects including adults under 50 years old and over 60 years old with normal hearing, and adults under 50 years old and over 60 years old with hearing loss. Performance was measured in anechoic and reverberant listening environments at two stimulus levels ( $25 \mathrm{~dB}$ SL and $70 \mathrm{~dB}$ SPL). Head-related transfer functions were used to create spatialized stimuli that were presented to subjects via earphones. Results indicate that performance on both tasks is affected by age and hearing loss. Localization errors are larger in older adults than younger adults with normal hearing, and larger in older adults with hearing loss than older adults with normal hearing. The intelligibility gain is smaller in older adults than younger adults with normal hearing, and smaller in older adults with hearing loss than older adults with normal hearing. Speech understanding in noise shows a large effect of reverberation, but localization is not affected by the same amount of reverberation. Overall, results suggest that in realistic environments these tasks are more difficult for older adults. [Work supported by the American Hearing Research Foundation.]

\section{1:40}

3aPPa11. Localization of distracted sound sources: Determining the role of binaural cues using unilaterally attenuated and interaurally uncorrelated signals. Jonas Braasch and Klaus Hartung (Inst. of Commun. Acoust., Ruhr Univ., D-44780 Bochum, Germany, braasch@ika.ruhr-uni-bochum.de)

Six listeners were instructed to localize manipulated random noise (200-ms duration, 20-ms $\cos ^{2}$-ramp, 12 directions in the horizontal plane) in an auditory virtual environment using individual head-related transfer functions (HRTF). In the first condition, the left headphone channel was attenuated by $20 \mathrm{~dB}$. In the second condition, an interaurally uncorrelated noise was used as the test signal by filtering two uncorrelated noises with the left and right HRTF. A second random noise was used as a distractor (500-ms duration, 20-ms $\cos ^{2}$-ramp, $0^{\circ}$ azimuth). The distractor was manipulated in the same way as the test signal. Four different signal-to-noise ratios $(\mathrm{S} / \mathrm{N}): 0,-5,-7$, and $-10 \mathrm{~dB}$ (plus an unmasked condition) were tested. In condition 1, the additional level difference of $20 \mathrm{~dB}$ caused a lateral displacement of the auditory event in four listeners. This displacement decreased when the distractor was added. In condition 2 (uncorrelated noise), the listeners were able to estimate the left-right direction, but frequently showed front-back confusions. With decreasing $\mathrm{S} / \mathrm{N}$ the perceived directions clustered at $-90^{\circ}, 0^{\circ}$, and $90^{\circ}$ azimuth.

12:00

3aPPa12. Localization ability in the horizontal plane in adverse auditory environments. Nancy L. Vause (U.S. Army Res. Lab., Human Res. and Eng. Directorate, Aberdeen Proving Ground, MD 21005) and D. Wesley Grantham (Vanderbilt Univ. Medical Ctr., Nashville, TN 37212)

The purpose of this investigation was to examine the effects of adverse conditions on localization ability in the horizontal plane. The experiment was designed specifically to assess horizontal plane localization accuracy for a target signal presented in the adverse conditions of noise, headgear, and reverberation for two orientations. Eight normal hearing subjects participated in a source identification task in nine different experimental conditions of headgear, environment, and background noise for the frontal and lateral orientation. The results showed that (a) the effects of noise degraded localization performance in both the frontal and lateral orientations to a greater extent than reverberation or the combination of reverberation + noise; (b) subject performance was not significantly disrupted by reverberation in a quiet environment, but did slightly decrease when noise and reverberation were combined; however, performance in the lateral orientation improved in both quiet and noise conditions; and (c) localization accuracy declined when subjects wore protective headgear in all conditions for both orientations. [Work supported by NICDC and U.S. Army.] 
Session 3aPPb

\title{
Psychological and Physiological Acoustics: Spectral Parameters (Poster Session)
}

\author{
Prudence Allen, Chair \\ School of Communication Sciences and Disorders, University of Western Ontario, 1500 Elborn College, London, \\ Ontario N6G 1H1, Canada
}

\section{Contributed Papers}

\begin{abstract}
All posters will be on display in the Poster Gallery from Monday to Wednesday, 15-17 March. Authors will be at their posters from 10:00 a.m. to 12:00 noon on Wednesday, 17 March.
\end{abstract}

\begin{abstract}
3aPPb1. The lower limit of melodic pitch with filtered harmonic complexes. Daniel Pressnitzer, Roy D. Patterson, and Katrin Krumbholz (CNBH, Dept. of Physiol., Downing St., Cambridge CB2 3EG, UK, daniel.pressnitzer@mrc-cbu.cam.ac.uk)

Sounds with temporal regularities can evoke a variety of percepts: pitch, roughness, flutter, or rhythm, according to the frequency range of the regularity. However, only pitch supports the perception of melodies. In an effort to determine the lower limit of pitch, as opposed to flutter, etc., an experiment has been performed to study melody perception with very low notes, as a function of frequency region and phase. These bandpassfiltered harmonic complexes had cosine, alternating, or positive-Schroeder phase. Listeners had to detect differences between two successive fournote melodies that where built around a base pitch. The lowest base pitch at which listeners could perform the task depended on both frequency region and phase. When the combination tone region was masked by low-pass noise, the phase dependence was strongly reduced. The lower limit of pitch increased markedly with frequency region when the masking noise was present. The results are discussed with regard to time-domain models of pitch involving autocorrelograms or auditory images. These models suggest the presence of precise pitch cues in high-frequency regions which appear to be contradicted by the current data. [Work supported by the Fyssen Foundation and the UK MRC (G9703469).]
\end{abstract}

3aPPb2. Further examination of the relationship between overshoot and filtering. Elizabeth A. Strickland (AUS Dept., Purdue Univ., West Lafayette, IN 47907, estrick@purdue.edu)

In a previous experiment, threshold was measured for a brief duration probe positioned temporally at the onset of a broadband masker, with and without a broadband precursor, to determine the amount of overshoot. The probe level was then fixed $10 \mathrm{~dB}$ above the threshold levels determined in the overshoot experiment, and a notched-noise masker was varied in level to estimate filter shape, with and without a precursor. In general, filters were broader and the signal-to-noise ratio at threshold, $k$, was lower following a precursor, but there was not a clear relationship between the amount of change in the filters and overshoot. In the present study, filter shapes were measured with the probe level set at the threshold from the overshoot experiment, rather than $10 \mathrm{~dB}$ above. Results revealed that the change in $k$ is in general closely related to the amount of overshoot (which would be expected) and also that there is at least some relationship between the change in filter width and the amount of overshoot. The study was also extended to look at possible filtering changes with decrease in overshoot, and to look at effects of overshoot from off-frequency precursors and maskers. [Work supported by NIH (NIDCD).]
3aPPb3. Frequency discrimination following linear frequency glide. Takuro Kayahara (Intelligent Modeling Lab., Univ. of Tokyo, 2-11-16 Yayoi, Bunkyo-ku, Tokyo 113-8656, Japan) and Takao Sato (Univ. of Tokyo, Tokyo 113-0033, Japan)

Difference limen for frequency (DLF) following linear frequency glide was measured using 2IFC procedure to investigate mechanisms underlying detection of frequency change in successive tones. In the experiment, a pair of 100-ms tone, one of which had fixed frequency of $1000 \mathrm{~Hz}$, was presented. The interval between the two pulses was $200 \mathrm{~ms}$ and the order of pulses in each trial was randomized. An adapting tone preceded each pair by $200 \mathrm{~ms}$ and was either 200-ms linear frequency glide with frequency extent of $100 \mathrm{~Hz}(950-1050 \mathrm{~Hz})$ or 200-ms stationary tone of $1000 \mathrm{~Hz}$. In the result, when the glide preceded the pair of pulses, DLFs at $1000 \mathrm{~Hz}$ were significantly elevated irrespective of direction of glide compared to DLFs with no preceding tones. The stationary sine wave, however, had no effect on DLFs as several previous studies have reported. In sum, unidirectional continuous change of frequency disrupted a process of sampling and comparing frequencies from successive tones. These results indicate that the process of detecting continuous and successive change of frequency are at least partially mediated by a common mechanism.

3aPPb4. Notched-noise measures of frequency resolution in children: What acoustic cues are available at children's threshold levels? Prudence Allen and Louise Korpela (Univ. of Western Ontario, School of Commun. Sci. and Disord., Elborn College, London, ON N6G 1H1, Canada, pallen@julian.uwo.ca)

It has been suggested that young children's masked detection thresholds are obtained at signal-to-noise ratios for which the signals produce changes in multiple features of the masker [Allen et al., J. Acoust. Soc. Am. (in press)]. In this study 500-, 2000-, and 4000-Hz tonal signals were added to flat- and notched-spectrum noise maskers at signal-to-noise ratios corresponding to children's threshold levels measured in a previous study of frequency resolution [Allen et al., J. Speech Hear. Res. 32, 317-324 (1989)]. The magnitudes of level, frequency, and periodicity cues were determined, assuming several degrees of frequency selective filtering. Results suggested that for all signal plus masker combinations, independent of frequency and masker spectrum, children's thresholds had been obtained at signal-to-noise ratios for which little or no frequency selectivity would have been required to produce potentially discriminable level differences [Jensen and Neff, Psychol. Sci. 4, 1-4-107] of approximately 6 $\mathrm{dB}$, which were often accompanied by average frequency and/or periodicity differences. Age-related changes in frequency selectivity therefore may not be required to explain children's higher masked detection thresholds as they are obtained at signal-to-noise ratios where multiple cues are present. [Work supported by NSERC.] 
3aPPb5. Effects of practice on performance for different masking tasks. Roel Delahaye, Deb A. Fantini, and Ray Meddis (Dept. of Psych., Essex Univ., Wivenhoe Park, Colchester CO4 3SQ, UK)

The present study investigates the effects of long-term practice on masked signal detection with experienced listeners. The on-frequency masker (OFM) consists of a $700-\mathrm{Hz}$ pure tone, which is sinusoidally amplitude-modulated at a $10-\mathrm{Hz}$ rate. Similar flanking components can be placed at 300, 400, 500, 900, 1000, and $1100 \mathrm{~Hz}$. The signal is composed of three, $50-\mathrm{ms}, 700-\mathrm{Hz}$ tone bursts that are placed in the dips of the OFM envelope. The flanking component envelope phases were either in phase (comodulated condition) or $180^{\circ}$ out of phase (uncorrelated condition) with the OFM. In the reference condition only the OFM was present. Two subjects gained experience $(20 \mathrm{~h})$ before the first data collection session took place. After extended practice $(40 \mathrm{~h})$ a second session took place. The results of the first session show a release from masking in the comodulated condition with regard to the reference condition (CMR R-C) and the uncorrelated condition (CMR U-C). Results from the second session show that reference thresholds decrease more than the comodulated and uncorrelated thresholds. This resulted in a reduction or disappearance of $\mathrm{CMR}(\mathrm{R}-\mathrm{C})$ but a preservation of $\mathrm{CMR}(\mathrm{U}-\mathrm{C})$. Thus the amount of practice is inversely related to the magnitude of $\mathrm{CMR}(\mathrm{R}-\mathrm{C})$, while $\mathrm{CMR}(\mathrm{U}-\mathrm{C})$ is independent of practice.

3aPPb6. Simulations of spectral masking and the asymmetry of masking. Jesko L. Verhey and Torsten Dau (Graduiertenkolleg Psychoakustik and AG Medizinische Physik, Univ. Oldenburg, Germany)

Measurements and simulations of (i) notched-noise experiments and (ii) masking experiments where the signal and the masker have the same center frequency but different bandwidths, are presented. The simulations were performed with a model originally developed to describe effects of temporal masking and modulation detection [Dau et al., J. Acoust. Soc. Am. 102, 2892-2905 (1997)]. The model accounts well for notched-noise data which are mainly a consequence of the gammatone-filter parameters used in the peripheral filter stage of the model. The model also accounts for effects in notched-noise experiments with an asymmetric notch ("offfrequency listening"), which results from the decision stage that integrates signal information across frequency. A simple modulation-lowpass filter (energy integrator) would be sufficient to describe these experimental results. In contrast, the processing by a modulation filterbank is necessary to account for the conditions of "asymmetry of masking" in the second experiment.

3aPPb7. Spectral integration of auditory temporal information. David A. Eddins (Psychoacoust. Lab., Dept. of Speech and Hearing Sci., Indiana Univ., Bloomington, IN 47405)

Measures of temporal acuity reveal a remarkable ability to integrate temporal information across frequency throughout most of the audible spectrum. This apparent trading of spectral bandwidth and temporal resolution was investigated in three different experiments measuring amplitude-modulation detection, temporal gap detection, and intensity discrimination. Each experiment included six spectral conditions: a single band of noise $1600-\mathrm{Hz}$ wide centered at $3000 \mathrm{~Hz}$; a set of four noise bands $400-\mathrm{Hz}$ wide having center frequencies separated by $1200 \mathrm{~Hz}(1200,2400$, $3600,4800 \mathrm{~Hz}$ ); and four conditions composed of only one of the four $400-\mathrm{Hz}$ bands. The form of the modulation transfer functions did not depend upon total bandwidth or frequency region. Sensitivity to modulation was inversely related to total noise bandwidth. Temporal gap detection thresholds and intensity discrimination were strongly dependent upon total noise bandwidth but were independent of the spectral proximity and the center frequency of the noise bands. Analysis of the three experiments revealed that the inverse relation between intensity discrimination and noise bandwidth may be used to predict the variation in amplitude modulation and temporal gap detection thresholds with noise bandwidth.
3aPPb8. Direction discrimination for formant-frequency glides and modulation. Johannes Lyzenga and Robert P. Carlyon (MRC Cognition and Brain Sci. Unit, 15 Chaucer Rd., Cambridge CB2 2EF, UK)

The vowel properties that determine their classification are the positions of peaks in their frequency spectra: the formants. For normal-hearing subjects, the sensitivity of detecting linear glides in the positions of these formants and the ability to discriminate between the two possible directions of these glides as functions of their sizes, were measured. A frequency rove was used to prevent subjects from using the start and end points of the glides as cues. The same quantities were measured for a $5-\mathrm{Hz}$ formant-frequency modulation. A three-interval, three-alternative forcedchoice paradigm was used in all measurements. The stimuli were singleformant "vowels" generated for a number of different stimulus parameters, including fundamental, spectral slope, frequency region, and position of the formant relative to the harmonic spectrum. For most stimuli, sensitivity was approximately equal for detection (of glides and modulation) on the one hand, and direction discrimination on the other. Exceptions were found for a few "formants" that consisted of a single harmonic that only showed an amplitude change over the stimulus length. The detection of formant-frequency modulation can be disrupted by offfrequency modulated formants, and new data on this phenomenon for modulation-direction discrimination will be reported.

3aPPb9. Estimating the phase characteristic of the auditory system from masking by harmonic complexes. Jennifer J. Lentz and Marjorie R. Leek (Army Audiol. and Speech Ctr., Walter Reed Army Med. Ctr., Washington, DC 20307, jennifer_lentz@wramc1-amedd.army.mil)

As shown by several recent studies of positive and negative "Schroeder-phase" stimuli, masking by harmonic complexes is strongly affected by the temporal waveform of the masker [e.g., V. Summers and M. Leek, Hearing Res. 118, 139-150 (1998)]. Differences in masking by the Schroeder waves provide a starting point for estimating the phase response of the auditory system. In this study, the gradient of phase change across masker bandwidth was varied by altering the Schroederphase algorithm. Depending on signal frequency (ranging from 500 to $4000 \mathrm{~Hz}$ ), differences in masking could be $5-10 \mathrm{~dB}$ for phase gradients changing by only $10 \%$. The phase gradient that resulted in a minimum amount of masking varied systematically with signal frequency, with low frequencies masked least effectively by waveforms with rapidly changing component phases, and high frequencies by waveforms with more shallow phase gradients. This relationship between phase gradient and signal frequency is taken to reflect the phase response of the auditory filter at that frequency. The phase gradients associated with minimal masking at each frequency can be used to construct a piecewise-estimate of the phase characteristic of the auditory system for individual listeners. [Work supported by NIH DC00626.]

3aPPb10. Unexpected dependency of psychoacoustical sharpness on the bandwidth of noise. Ulrich Haupt and Reinhard Weber (Dept. of Phys., Carl-von-Ossietzky Univ. of Oldenburg, D-26111 Oldenburg, Germany)

Based on semantic differential and scaling experiments, von Bismarck [Acustica 30, 146-159 (1974)] proposed a model for psychoacoustical sharpness calculation, which later on was modified by Aures [Acustica 58, 282-290 (1985)]. Categorical sharpness evaluation of sounds exhibited systematic deviations from calculated sharpness after Aures under special conditions. Sharpness ratings of bandpass noises with the same calculated sharpness decreased if the bandwidth $\Delta B$ of the noises increased [E. Kabot and R. Weber, DAGA '94, 1029-1032 (1994)]. The intent of the present study was to investigate this effect in detail. The stimuli to be assessed consisted of two sets of sounds containing tones and broadband noises ( $\Delta B=0.5,1,2$, and 5 Bark). All sounds in a set had equal calcu- 
lated sharpness and were presented over headphones at the same loudness. Using a five-category scale, subjects evaluated each sound in two sessions, six times, in a random order. The results will be discussed against the background of new sharpness model calculations.

3aPPb11. Gap detection within and across perceptual channels. Andrew J. Oxenham (Dept. of Speech-Lang. Pathol. \& Audiol. (133 FR), Northeastern Univ., 360 Huntington Ave., Boston, MA 02115)

The ability to detect gaps is degraded when the stimuli (markers) on either side of the gap have different power spectra. This may be due to peripheral frequency selectivity: when no transient is audible in the no-gap condition (e.g., when the markers have the same intensity and spectrum), listeners detect the transient introduced by the gap; when the markers fall into different auditory filters or are at different intensities, transients are audible regardless of the presence of a gap and listeners must perform a timing judgment. It has recently been suggested that changes in other, more central, perceptual dimensions, such as perceived location, may also affect gap detection. This study was designed to test whether a peripherally based model could also account for such data. Lateralization differences between the two broadband-noise markers were achieved using interaural time and intensity differences independently. Monaural gap detection with level differences between the markers was also tested. Results indicate that gap detection is poor only when level differences exist between the markers, independent of perceived location. Thus the data can in principle be accounted for by peripheral mechanisms. No evidence was found for the influence of higher perceptual channels. [Work supported by the NIDCD.]

3aPPb12. Acoustic weapons? Effects of very high sound levels. Jürgen Altmann (Experimentelle Physik III, Universität Dortmund/ Institut für Experimentalphysik III, Ruhr Universität Bochum, 44780 Bochum, Germany, Altmann@EP3.Ruhr-Uni-Bochum.DE)

Mainly in the context of so-called nonlethal weapons, acoustic weapons are under research and development in a few countries. Reliable information is scarce; allegations, mainly in the military press, have attributed drastic effects in particular to infrasound. Humans would suffer from disorientation and nausea up to vomiting and uncontrolled defecation, however without lasting damage. For damage to material, embrittlement of metals and even localized earthquakes have been mentioned. A literature review was done to investigate such claims and find out other effects of strong sound in the infrasound and low-frequency audio, highfrequency audio, and ultrasound ranges, as well as of blast waves. Whereas the infrasound allegations cannot be confirmed, marked nonauditory effects exist in the low audio range at levels above $150 \mathrm{~dB}$. At higher frequencies and with blast waves, mainly in the auditory system is affected. With increasing level, annoyance, pain, and permanent damage ensue; protection is possible, however. Very strong blast waves can cause lung rupture and death. Because application scenarios range from protection of fixed installations via riot control to armed conflict, potential regulation should not be restricted to the law of warfare, and preventive limits will need to be detailed.

\title{
Session 3aSA \\ International Workshop on Active Noise and Vibration Control
}

\section{Structural Acoustics and Vibration: Active Control of Sound Radiation and Transmission}

\author{
Peter Dean, Cochair \\ Lockheed Martin Missiles and Space Org., H1-33, B203, 3251 Hanover Street, Palo Alto, California 94304, USA \\ Paolo Gardonio, Cochair \\ Signal Processing and Control Group, ISVR, University of Southampton SO17 1BJ, UK
}

Invited Paper

9:00

3aSA1. Experiments on active control of sound radiation around double-wall resonance frequency. S. J. Pietrzko (EMPASwiss Federal Labs. for Mater. Testing and Res., CH-8600 Dübendorf, Switzerland, pietrzko@empa.ch)

The first part of this paper will be focused on identified modal shapes and sound power measurements of a double-wall panel in the low-frequency range around the double-wall resonance frequency. New experimental results show that for a double-wall panel with one laminated pane (asymmetric panel) around the predicted double-wall resonance frequency, there exist three natural frequencies, respectively three modes. One of these modes is highly complex and the other two are real. It is characteristic that at one of these real modes both planes of the panel move exactly in phase and at the second they move precisely out of phase. The second part of the paper will be focused on the design of control system to minimize the radiated sound power from the double-wall panel and efficiency of the proposed control. The radiation efficiency for each of the identified modes was estimated with goal to define the optimal position for the piezoactuators used to minimize the radiated sound power around the double-wall resonance frequency. Different control schemes will be presented. The efficiency of proposed control schemes will be compared and illustrated by means of laser scanned vibratory patterns of the panel. 
3aSA2. Active control of harmonic and random sound transmission in a structural-acoustic system. M. J. Brennan and S. M. Kim (Inst. of Sound and Vib. Res., Univ. of Southampton, Southampton, Hampshire SO17 1BJ, UK, mjb@isvr.soton.ac.uk)

The active control of sound transmission in structural-acoustic coupled systems has been of interest in recent years. Two particular problems that have received attention are the active control of harmonic sound transmission into aircraft, and control of random noise transmission into the payload bay of space rockets. This paper describes an analytical and experimental investigation to compare the feedforward control of harmonic and random sound transmission into an acoustic cavity. A rectangular enclosure is considered that has five acoustically rigid walls and a flexible plate on the remaining side through which a plane acoustic wave is transmitted into the enclosure. The control systems are designed to reduce the acoustic potential energy inside the enclosure when the incident sound is either harmonic or random. Three control configurations, classified by the type of actuators, are investigated both theoretically and experimentally. They are the use of a structural actuator, the use of an acoustic source, and the simultaneous use of both structural and acoustic actuators. It is shown that the use of both actuators is desirable for the active control of both harmonic and random sound transmission into a coupled structural-acoustic system whose response is governed by plate and cavity controlled modes.

\section{9:40}

3aSA3. Near-field control of complex sources. Jiri Tichy (Grad. Prog. in Acoust., Penn State Univ., University Park, PA 16802)

The near-field of complex sources that are characterized by standing waves on their surface is modeled by baffled point sources. The near field is visualized by showing the spatial distributions of sound pressure, sound intensity, and energy density. The field maps provide essential information on the local energy flows. Several sound control schemes to minimize the total radiated sound power by placing small sources in the vicinity of the primary sources are analyzed and tested.

\section{0:00}

3aSA4. A comparison of active control strategies for the reduction of sound transmission through double panels. Paolo Gardonio and Stephen J. Elliott (Inst. of Sound and Vib. Res., Univ. of Southampton, Southampton, UK)

A theoretical study of the active isolation of air-borne and structureborne sound transmission through a double panel system is presented. The reductions in the radiated sound are investigated for three different families of control approaches. The first and the second families are based on the minimization of total sound power radiation or the minimization of the weighted sum of the square values of the sound pressure at a set of positions between the two panels and the square values of the velocities at a set of positions of the radiating panel using either active mounts or/and control loudspeakers. The third family considers: first, the cancellation of out-of-plane velocities underneath the mounts using active mounts; second, the cancellation of sound pressure near each loudspeaker using loudspeakers; and third, the cancellation of both velocities and the sound pressure using both loudspeakers and mounts. The third family does not give good performances except at low frequencies. Alternatively, the minimization of weighted square values of plate velocities and cavity pressures gives good results which are comparable with those obtained with the optimal control approach of total sound power minimization. In general, the control loudspeakers give better control in all control approaches considered.

\section{0:40}

3aSA5. Intensity error sensing in the active control of sound. Robert Koehler and Scott D. Snyder (Mech. Eng. Dept., Univ. of Adelaide, Adelaide, SA 5005, Australia)

In the active control of radiated sound fields, the use of intensity as an error signal has intuitive appeal, due to the fact that the measure is directly related to the sound power radiated from the source. To date, examinations of this proposition have been restricted largely to theoretical studies. This work to be presented here extends previously derived theoretical results, and presents experimental results from the use of intensity-based error measures in a practical adaptive active noise control system. It is found that while minimization of an acoustic pressure-based error signal does not guarantee a satisfactory global result, the use of an intensity-based error signal may not improve the situation. In fact, there are many instances where the use of a pressure-based error signal in the active noise control system may be preferable. This includes instances where the error signal is measured in the near field of the sound source, at locations where it was hoped that minimization of intensity-based error signals would deliver superior overall active control system performance.

\section{1:00}

3aSA6. Simulations of the feedback control of sound transmission through a plate using measured data. Thomas C. Sors and Stephen J. Elliott (Inst. of Sound and Vib. Res., Univ. of Southampton, Southampton, UK)

This paper describes a simulation of active structural acoustic control (ASAC) based on experimentally measured frequency response functions. An experimental arrangement consisting of a plate mounted between two rooms and equipped with integrated actuators and sensors is described. The aim of the experiment is to use these transducers mounted on the plate to reduce the transmission of an acoustic disturbance generated by a loudspeaker in one room to the other. Frequency response functions are measured from the loudspeaker and the actuators to the integrated sensors and also to a number of far-field error microphones. These measurements are used to predict the performance of various feedback controllers on the sound transmission. It is found that, provided there is only a small amount of delay inherent to the control process, significant reductions can be made for periodic disturbances and also some reductions can be made for broadband disturbances. [Work supported by the TNO Institute of Applied Physics, Delft, The Netherlands.]

\section{1:20}

3aSA7. Experiments on the active increase of sound transmission loss using distributed actuators. Kris Henrioulle and Paul Sas (Katholieke Univ. Leuven, Celestijnenlaan 300B, B-3001 Leuven, Belgium)

A series of experiments have been performed to improve actively the sound transmission loss through a single-panel partition in the lowfrequency range. Two types of flat acoustic actuators, designed in the framework of the European project DAFNOR (distributed active foils for noise reduction), are tested. The first acoustic actuator is based on PVDF piezoelectric material, which induces a bending motion in the actuator. The second acoustic actuator is based on the electrostatic principle. The experimental setup consists of a single steel panel of size $300 \times 400 \times 1$ $\mathrm{mm}$ which is subject to a plane-wave acoustic excitation. The control system consists of the distributed acoustic actuator, which is placed near the steel panel. Various control strategies, which were previously simulated, are tested. The control actuator is driven by a controller which aims at reducing the acoustic energy at the radiating side. [The work reported herein was related to the EC Brite/Euram Research Project "DAFNOR", (under contract BRPR-CT96-0154). The project is supported by the Directorate-General for Science, Research and Development of the CEC.] 
11:40

3aSA8. An experimental investigation of a novel active segmented partition for sound transmission control. Timothy W. Leishman $^{\text {a) }}$ and Jiri Tichy (Grad. Prog. in Acoust., Penn State Univ., 217 Appl. Sci. Bldg., University Park, PA 16802, leishman@sabine.acs.psu.edu)

A unique dual diaphragm configuration for individual elements of active segmented partitions has recently been advanced as a practical, efficient means of controlling sound transmission. The configuration incorporates a useful combination of electro-mechano-acoustical actuation and segmentation, enabling effective global control of transmitting surface vibrations. In this work, experimental efforts are reported that explore important active and passive characteristics of such elements while adjoined in a full partition array. Each element is independently and stably controlled using a single-channel filtered-x adaptive controller (single reference input, single error input, single output). Acoustic pressure scans of element cavities and laser vibrometer scans of their transmitting surfaces affirm important properties of this partition construction that have been previously theorized. Other measurements demonstrate that the controlled transmission loss of the lightweight array lies uniformly between 55 and $60 \mathrm{~dB}$ over nearly all of the evaluated spectrum $(30$ to $300 \mathrm{~Hz})$. Such extensive control is generally very difficult to realize over the lower audible frequency spectrum using an extended lightweight structure. Further measurements illustrate that this is comparable to the passive transmission loss of a much more massive benchmark partition of the same crosssectional area, but constructed of thick, spaced layers of concrete and sand. ${ }^{a)}$ Currently at Crown International, 1718 W. Mishawaka Rd., Elkhart, IN 46515-1000.

\section{2:00}

3aSA9. A hybrid passive/active system for control of the noise radiated by a small generator. Maria Cuesta and Pedro Cobo (Instituto de Acustica, CSIC, Serrano 144, 28006 Madrid, Spain, iacpc24@fresno.csic.es)

The aim of this work was the design and implementation of a hybrid passive/active system to control the noise radiated by a small generator. The fundamental frequency of the periodic radiated noise depends on the electrical load plugged into the generator. Passive control is afforded by a rectangular enclosure with dimensions $1000 \times 710 \times 530 \mathrm{~mm}^{3}$. The wall panels of the enclosure (steel $1.5 \mathrm{~mm}$ thick) are lined with a $30-\mathrm{mm}$ layer of sound absorbing material. Special attention is paid to technical aspects such as air refreshing and temperature inside the enclosure. The measured insertion loss is higher than $20 \mathrm{~dB}$ above $500 \mathrm{~Hz}$. Low-frequency noise escapes the enclosure via intake and exhaust openings. Active control is applied to the exhaust noise. The reference signal is supplied by an accelerometer over the air filter case of the generator. A high-temperature loudspeaker is used as control source. The error signal is picked up by a cheap electric microphone. Since noise along the exhaust is too loud, the microphone sensitivity had to be reduced by taping its diaphragm. A commercial system is used to implement active noise control. [Work supported by CICYT, Project AMB97-1175-C03-01.]

WEDNESDAY MORNING, 17 MARCH 1999

ROOM H3010, 7:55 A.M. TO 12:20 P.M.

\title{
Session 3aSCa
}

\section{Speech Communication: Psychoacoustic Methods in Speech Recognition I}

\author{
Birger Kollmeier, Cochair \\ AG Medizinische Physik, Universität Oldenburg, Postfach 2503, D-26111 Oldenburg, Germany \\ Hynek Hermansky, Cochair \\ Oregon Graduate Institute of Science and Technology, Beaverton, Oregon 97006-8921, USA
}

Chair's Introduction-7:55

Invited Papers

\begin{abstract}
8:00
3aSCa1. What can we learn from human processes of spoken language comprehension? Hiroya Fujisaki (Dept. of Appl. Electron., Sci. Univ. of Tokyo, 2641 Yamazaki, Noda, 278-8510 Japan, fujisaki@te.noda.sut.ac.jp)

Conventional approaches for automatic speech recognition and understanding are essentially bottom-up. Namely, they are based on the assumption that speech can be decomposed reliably into the smallest units, can be recognized by identifying these units first, and then by clustering them to find larger units and their interrelationships. It is thus taken for granted that speech recognition is the prerequisite to speech understanding. The human processes of spoken language comprehension, on the contrary, are rather holistic and do not necessarily require identification of every constituent unit. This is possible since the listener has the capability of constructing the expected message while listening, and of matching it against the input message on the basis of global features, without paying much attention to the details. In the author's view, the unit of human speech processing is considered to be not fixed, but variable in size depending on the context, situation, and knowledge. Likewise, the human processes of lexical access and parsing are considered to be quite different from those used in conventional systems for natural language processing. These issues will be discussed on the basis of studies by the author and his co-workers as well as by others.
\end{abstract}


3aSCa2. A psychoacoustical model of the auditory periphery as the front end for ASR. Jürgen Tchorz, Michael Kleinschmidt, and Birger Kollmeier (AG Medizinische Physik, Univ. Oldenburg, Oldenburg, Germany)

A psychoacoustical model of the auditory periphery was developed by Dau and others to predict human performance quantitatively in typical spectral and temporal masking experiments. Its main processing stages are basilar membrane filtering for spectral analysis, adaptive envelope compression in each frequency band which enhances changes in the input signal and suppresses steadystate portions, and low-pass filtering of envelope modulations. In the field of speech processing, the model was applied as the front end of an automatic speech recognition (ASR) system [J. Tchorz and B. Kollmeier, J. Acoust. Soc. Am. (submitted)]. Speakerindependent, isolated-digit recognition experiments in different types of noise were carried out to evaluate the robustness of the auditory-based ASR system in adverse conditions. Compared to a standard MFCC front end, the auditory-based preprocessing yielded significantly higher recognition rates in both additive and convolutive noise. Further experiments concentrated on the question, which of the processing stages of the psychoacoustically motivated model are essential for robust representation of speech? The results indicate that the adaptive compression stage and low-pass filtering of envelope modulations contribute most to robustness, whereas the spectral analysis part is less critical.

\section{8:40-9:00 Discussion}

\section{9:00}

3aSCa3. Automatic classification of the acoustical situation using amplitude-modulation spectrograms. Jürgen Tchorz and Birger Kollmeier (AG Medizinische Physik, Univ. Oldenburg, Oldenburg, Germany)

A fast and reliable classification of the acoustical situation is an important prerequisite for modern speech processing schemes. Noise suppression algorithms in digital hearing aids, for example, are strongly dependent on a proper noise level estimation. The classification algorithm which is presented is based on so-called amplitude-modulation spectrograms (AMS). It is motivated by neurophysiological findings in the auditory cortex in mammals. Its basic idea is that both spectral and temporal information of the signal is used to attain a separation between "acoustical objects" within the signal. Spectral information is gained by splitting the input signal into different frequency bands, temporal information is gained by analyzing amplitude modulations in each frequency band. Thus for each analysis frame of the input signal, a two-dimensional AMS pattern is created. Signals with similar spectral shape but different temporal properties are clearly distinct in the AMS representation. A neural network is trained on a large number of AMS patterns which were generated from speech and noise samples. After training, an automatic classification of speech and noise from "unknown" sources is performed with high accuracy. Furthermore, an estimate of the present signal-to-noise ratio is supplied for mixed input signals.

9:20

3aSCa4. Evaluation of PEMO in robust speech recognition. Klaus Kasper and Herbert Reininger (Inst. für Angewandte Physik, Goethe Univ., Robert-Mayer-Str. 2-4, 60054 Frankfurt, Main, Germany)

A major problem in speech recognition is the robustness of recognition performance against additive background noise and convolutive distortions due to changing transmission channels or varying microphone characteristics. One approach to overcome this problem is to apply PEMO [Dau, Püschel, and Kohlrausch, J. Acoust. Soc. Am. 99, 3615-3622 (1996)] for extraction of noise robust feature vectors. The recognition performance achievable with PEMO in combination with a locally recurrent neural network (LRNN) or hidden Markov models (HMM) for feature scoring was evaluated in a task of speaker-independent word recognition. The robustness was tested and compared with that of other feature types by applying the speech recognition systems to speech signals disturbed by additive background noise and to speech signals recorded over telephone lines. It was found that only with LRNN it is possible to exploit the potential of PEMO, not with HMM. A detailed analysis of the interplay between PEMO features and the scoring techniques revealed that the long-term dependencies in the sequence PEMO features can only be captured by LRNN. Furthermore, LRNN discriminate between the distinct and sparse peaks of the PEMO speech representation-which are well maintained also in the case of noisy speech—and artifacts introduced by the additive or convolutive noise.

9:40-10:00 Discussion

10:00-10:20 Break

10:20

3aSCa5. Syllable-based speech recognition using auditorylike features. Steven Greenberg, Takayuki Arai, Brian Kingsbury, Nelson Morgan, Michael Shire, Rosaria Silipo, and Su-Lin Wu (International Computer Science Inst., 1947 Center St., Berkeley, CA 94704)

Classical models of speech recognition (both human and machine) assume that a detailed, short-term analysis of the signal is essential for accurate decoding of spoken language via a linear sequence of phonetic segments. This classical framework is incommensurate with quantitative acoustic/phonetic analyses of spontaneous discourse (e.g., the Switchboard corpus for American English). Such analyses indicate that the syllable, rather than the phone, is likely to serve as the representational interface between sound and meaning, providing a relatively stable representation of lexically relevant information across a wide range of speaking and acoustic conditions. The auditory basis of this syllabic representaion appears to be derived from the low-frequency $(2-16 \mathrm{~Hz})$ modulation spectrum, whose temporal properties correspond closely to the distribution of syllabic durations observed in spontaneous speech. Perceptual experiments confirm the importance of the modulation spectrum for understanding spoken language and demonstrate that 
the intelligibility of speech is derived from both the amplitude and phase components of this spectral representation. Syllable-based automatic speech recognition systems, currently under development, have proven useful under various acoustic conditions representative of the real world (such as reverberation and background noise) when used in conjunction with more traditional, phone-based recognition systems.

\section{0:40}

3aSCa6. The profile of interaural decorrelation across frequency: A binaural representation of pitch and timbre. A. Quentin Summerfield (MRC Inst. of Hearing Res., Univ. of Nottingham, Nottingham, UK), John F. Culling (Univ. of Wales, Cardiff, UK), and Michael A. Akeroyd (Univ. of Nottingham, UK)

Tones can be detected in noise at lower signal-to-noise ratios (SNRs) when they possess different interaural timing to the noise. One account, equalization-cancellation (E-C), suggests that the left- and right-ear signals are adjusted in time and amplitude to maximize their similarity, and are then cancelled by subtraction. At low SNRs, E-C improves the SNR of the tone by cancelling the noise. E-C is also a method for measuring interaural decorrelation, because the remainder after subtraction is a measure of the extent to which the tone reduces the interaural correlation of the noise. Applying E-C independently in each frequency channel reveals the profile of interaural decorrelation across frequency induced by a complex signal in noise. The profile is a place representation akin to an excitation pattern. It has three properties. (i) It displays the spectrum of speech at low SNRs. (ii) Listeners can integrate it perceptually with the conventional profile of spectral amplitude. (iii) Its spectral structure is compatible with a subset of the dichotic pitches phenomena in which tonal percepts are induced by introducing interaural phase changes into broadband noises. Finally, the profile can be recast in a form compatible with temporal accounts of pitch and timbre based on autocorrelation.

\section{1:00-11:20 Discussion}

\section{1:20}

3aSCa7. Recognition of speech from temporal patterns. Sangita Sharma and Hynek Hermansky (Oregon Grad. Inst. of Sci. and Technol., Portland, OR, hynek@ece.ogi.ed)

Spectrum of short segments of speech signal is accepted as one of the prime acoustic correlates of phonetic quality and it forms the basis of most current feature representations of speech. However, the short-term spectral vectors are affected by coarticulation. The coarticulation can span time intervals of several phonemes. In spite of that, human speech perception is able to derive the identity of the individual underlying phoneme. This process does not seem to require successful decoding of neigboring phonemes [Allen, IEEE Trans. AU-2(4), 567-577 (1994)], but it may require information from a relatively large temporal span of the speech signal [Kozhevnikov and Chistovich, "Speech: Articulation and Perception," Dept. of Commerce (1967)]. To allow for effective use of a syllable-level temporal information, a short-term spectrum-based vector is replaced as input to the classifier by rather long (about $1 \mathrm{~s}$ ) vectors representing the time trajectory of critical-band spectral energy. Each temporal vector then spans many more than one phoneme. This technique forms the basis of a complete ASR system which is competitive with the current state-of-the-art speech recognition technology. [Work supported by the Department of Defense and by the National Science Foundation.]

\section{1:40}

3aSCa8. Some musings on systematic variability and speech recognition. Jordan Cohen (Ctr. for Commun. Res., Princeton, NJ 08502)

One of the major problems in speech recognition is the inability of trained models to generalize appropriately to channel variations, new speakers, or modified acoustics. The naive observer would believe that a multimillion-parameter system should be sufficient! The difficulty appears to be too many parameters rather than too few. For moderate-sized training corpora, systems learn all of the conditions in the training data rather than generalizing from the exemplars. (For instance, speech recognition algorithms will generally score the speech from a training speaker higher than that from a speaker who was excluded from the set.) One can force the issue by explicitly modeling systematic variation, and then "normalizing" at the front end or in the acoustic model. Two exemplars of this philosophy are Cepstral mean subtraction and vocal tract normalization [Frontiers in Speech Processing 94, LDC96s40, Linguistic Data Consortium (1995)]. In each case a single parameter from a very restrictive model is estimated, and accounting for the variability explicitly improves performance. Concrete examples of these situations are offered, and the impact of this work on future work in automatic speech recognition is discussed.

12:00-12:20 Discussion 


\title{
Session 3aSCb
}

\section{Speech Communication: Fluid Dynamic Models for Speech Production}

\author{
Anders Lofqvist, Cochair \\ Haskins Laboratories, 270 Crown Street, New Haven, Connecticut 06511, USA \\ Christine H. Shadle, Cochair \\ Department of Electronics and Computer Science, University of Southampton, Highfield, Southampton SO17 1BJ, UK
}

Chair's Introduction-7:55

Invited Papers

\begin{abstract}
8:00
3aSCb1. Fluid dynamic effects in speech. Michael Krane (CAIP Ctr., Rutgers Univ., Piscataway, NJ 08855, mkrane@ caip.rutgers.edu)

A summary of fluid dynamic effects in speech are outlined using concepts from duct flow aerodynamics and aeroacoustics. A physical picture of fluid motion which produces both voiced and unvoiced sounds is presented, with emphasis on how movement of the acoustic medium affects both the generation and propagation of sound. Particular attention is given to the concept of vorticity (roughly, fluid particle rotational motion) as a means to characterize turbulent flow and as the means by which airflow produces or absorbs sound. First, it is shown how vorticity production by steady flow at the end of an open pipe may act as an absorber of sound, affecting the acoustic impedance of the open end. Unvoiced sound production is then discussed in terms of the formation of a jet by flow separation, the structure of jet vorticity, and how convection of jet vorticity through a nonuniform vocal tract produces sound. Order-of-magnitude estimates of these effects are presented. Finally, the role of these effects in voice production are detailed through a discussion of the modulation of glottal volume flow by movement of the glottal flow separation point and production of sound by the glottal jet.
\end{abstract}

8:20

3aSCb2. Turbulent glottal flow during phonation. Fariborz Alipour (Dept. of Speech Pathol. and Audiol., The Univ. of Iowa, Iowa City, IA 52242)

Human phonation is the flow-induced oscillation of the vocal folds, producing pulsatile jet through the glottis, which may become turbulent. This turbulent jet was investigated in an excised canine larynx model with simultaneous recordings of air particle velocity, subglottal pressure, airflow rate, and EGG signal for various conditions of phonation. Canine larynges were mounted on a plastic air tube and sustained oscillations were established and maintained by mechanically manipulating the cartilages to mimic the function of laryngeal muscles. The major control parameters were the airflow rate and the glottal adduction. The velocity was measured with a constant-temperature hot-wire anemometer system. To separate the turbulence from the periodic component of the velocity signal, the technique of phase shift averaging was employed. Results suggest that subglottal inlet flow to the larynx is pulsatile but mostly laminar, while the exiting jet is nonuniform and turbulent. The Reynolds number based on the mean glottal velocity and glottal hydraulic diameter varied between 1600 and 7000, the Strouhal number varied between 0.002 and 0.032 , and the Womersley number ranged from 2.6 to 15.9. These results help define the conditions required for computational models of laryngeal flow. [This work was supported by NIDCD Grant No. DC03566-01.]

\section{8:40}

3aSCb3. A study of the supraglottal flow behavior during phonation. Xavier Pelorson, Coriandre Vilain (ICP/INPG, Univ. Stendhal, 46 av. F. Viallet, F-38031 Grenoble, France), and Avraham Hirschberg (Tech. Univ. Eindhoven, Eindhoven, The Netherlands)

Most physical models of phonation assume the formation of a free jet at the end of the glottis. The precise behavior of this jet is generally overlooked, assuming a symmetrical flow and a total dissipation of the kinetic energy past the glottis. However, asymmetry of the glottal flow (Coanda effect) and false folds effects (reattachment of the jet) have been observed on the basis of quasi-steady flow numerical simulations. In this paper the relevance of these effects on the basis of unsteady in-vitro measurements using pulsating flows through symmetrical models of the glottis is discussed. It is shown that asymmetry of the flow due to the Coanda effect is typical of steady flows but does not have time to be established during unsteady conditions. An extension of this study concerning effects of an asymmetry of the glottis will also be discussed. Concerning the effects of the false folds, many parameters have been studied such as the width of the false folds, and the distance between the true and the false folds. It is shown that for typical phonation configurations the possible effects of the false folds remain very weak. This might not be the case for pathological configurations. 
3aSCb4. Models of frication: Advances and limitations. Christine H. Shadle (Dept. of Electron. and Computer Sci., Univ. of Southampton, Southampton SO17 1BJ, UK, chs@ecs.soton.ac.uk)

The increasing number of modeling studies of fricatives and frication in recent years is due at least partly to two major advances: increasing computational power makes it feasible to simulate the flow, and thus construct a more physically accurate model; improvements in vocal-tract imaging, particularly magnetic resonance imaging (MRI), allow capture of three-dimensional vocal-tract shape data, especially including small constrictions formed by soft tissue. However, the complexity of both techniques still tends to lead to incomplete models of frication, limited to static and/or highly simplified tract shapes. Further, the three-dimensional data are often used only to generate an area function, ignoring the cross-sectional shape data that can be crucial in defining aeroacoustic sources. Fricatives are not static, and some of their dynamic behavior, such as apparent modulation of the noise source in voiced fricatives, may be especially ill-served by quasistatic models. Dynamic MRI techniques can be used to acquire shape data with which to pursue dynamic models. Finally, the program VOAC will be discussed briefly (and in more detail elsewhere in this session) as an example of a way to use three-dimensional data and acoustic-flow interactions to enhance the model without unduly increasing computational, or conceptual, complexity.

\section{9:20}

3aSCb5. Computational aeroacoustics of subsonic jets for application to speech production. S. H. Frankel, W. Zhao, L. Mongeau (School of Mech. Eng., Purdue Univ., West Lafayette, IN 47907), and R. C. Scherer (Bowling Green State Univ., Bowling Green, $\mathrm{OH} 43403)$

Computational aeroacoustics calculations, based on direct numerical simulations, were performed in order to predict the near-field and far-field sound radiated by a forced, round, unconfined, subsonic jet. The jet Mach number was around $\mathrm{Ma}=0.4$, and the Reynolds number based on the jet velocity and diameter was approximately $\mathrm{Re}=103$. The compressible Navier-Stokes equations were integrated using a high-order finite-difference scheme. A two-dimensional, axisymmetric, nonuniform grid was used. Both direct computations and acoustic analogies were used to compute the far-field sound. Of interest is the role of large-scale vortical flow structures and vortex pairing in the production of sound. The results were used to assess the validity and limitations of the computational approach through comparisons to previously published numerical results. The ultimate goal is to perform large eddy simulations of pulsating jets in a range of flow conditions more relevant to speech production, e.g., approximately $\mathrm{Ma}=0.1, \mathrm{Re}=105$, to establish the radiation efficiency and the physical origin of the aeroacoustic sources involved. [Work sponsored by NIH.]

\section{9:40}

3aSCb6. Synthesis of unvoiced speech sounds using an aeroacoustic source model. Michael Krane, Daniel Sinder, and James Flanagan (CAIP Ctr., Rutgers Univ., Piscataway, NJ 08855, mkrane@ caip.rutgers.edu)

An aeroacoustics-based method for synthesizing unvoiced speech sounds is presented. The source model is that of Howe (1975) for sound generated by flow of vorticity through a nonuniform duct. In this formulation three quantities must be known: the potential flow velocity, which changes according to the duct shape, the vorticity, and the velocity induced by vorticity in the flow and its images in the duct walls. The potential flow velocity magnitude is found using a transmission-line representation of the vocal tract, and the direction is found using a panel method. A model for the generation and evolution of vorticity has been developed in which the vortex shedding frequency is a random variable, and the vortex convects at a constant speed and direction. The computational model was used to simulate three cases: (1) a jet flowing through a pipe constriction, (2) a fricative sound (/s/), and (3) a VCV combination (/a s a/). Simulation results are presented and compared to experimental data, in terms of spectra and listening tests.

10:00-10:20 Discussion

10:20-10:40 Break

\section{Contributed Papers}

10:40

3aSCb7. A computational study of glottal obliquity. Ronald C. Scherer (Dept. of Commun. Disord., Bowling Green State Univ., Bowling Green, OH 43403), Kenneth J. De Witt, Abdollah A. Afjeh, Chao Zhang (Univ. of Toledo, Toledo, OH 43606), and Steven H. Frankel (Purdue Univ., West Lafayette, IN 47907)

Glottal motion is not always symmetric during phonation. The cycle phase of one vocal fold may differ from that of the other, creating a dynamic glottal center line oblique to the tracheal center line. This condition is called "obliquity" of the glottis. Intraglottal pressures for a range of obliquity angles were obtained using a finite difference computational technique for steady flow in static geometries. As an example, for a uni- form glottal duct, obliquity of $20 \mathrm{deg}$ (one side convergent, the other divergent), subglottal pressure of $7.8 \mathrm{~cm} \mathrm{H}_{2} \mathrm{O}$, and glottal diameter of $0.032 \mathrm{~cm}$ : (1) air pressures were significantly different between the two glottal walls (as large as approximately 24 pressures measured perpendicular to the tracheal axis, but less if measured perpendicular to the glottal duct, (2) pressure values were different in amount and even polarity from that expected for a uniform glottis with zero obliquity, and (3) there were significant pressure differences at glottal exit between the two sides. Aerodynamic equations for glottal flow need to be modified to account for obliquity. The computational pressure profiles will be compared to empirical results using a Plexiglas model (M5) of the larynx. [Work supported by NIH grant 1R01DC03577-01A1.] 
3aSCb8. Estimating the nonacoustic fluctuating velocity amplitude from Cranen and Boves' vowel production data. Anna Barney (Faculty of Eng. and Appl. Sci., Univ. of Southampton, Southampton SO17 1BJ, UK)

In a dynamic mechanical model of the larynx and vocal tract [Barney et al., J. Acoust. Soc. Am. (in press)], the fluctuating velocity field was shown to have contributions from an acoustic disturbance and also from nonacoustic flow associated with vortex transport along the model tract. Nonacoustic velocity fluctuations, interacting with changes in the tract area function, can constitute significant sources of sound; in the vocal tract these could occur at the lips, teeth, etc. However, such additional sources of sound are not generally included in models of voiced speech production. Cranen and Boves [J. Acoust. Soc. Am. 77, 1543-1551 (1985)] obtained in-tract and radiated sound-pressure time histories from phonating subjects, from which they computed in-tract velocities. In this study the relative amplitudes of the acoustic and nonacoustic velocity fluctuations have also been estimated from their data. Though vocal tract area functions are not available, simple models of sound transmission and radiation allow an order-of-magnitude prediction of the contribution to the radiated sound associated with each part of the in-tract velocity field. Although these are preliminary investigations using a restricted data set, the evidence suggests that there is nonacoustic fluctuating flow within the vocal tract, capable of significant sound generation.

\section{1:20}

3aSCb9. A model of vocal-tract acoustics and aeroacoustics validated by flow experiments. Philip J. B. Jackson and Christine H. Shadle (Dept. of Electron. and Computer Sci., Univ. of Southampton, Southampton SO17 1BJ, UK, pjbj96r,chs@ecs.soton.ac.uk)

Modeling the acoustic response of the vocal tract is a complex task, both from the point of view of acquiring details of its internal geometry and of accounting for the acoustic-flow interactions. A vocal-tract acoustics program (VOAC) has been developed [P. Davies, R. McGowan, and C. Shadle, in Vocal Fold Physics, edited by I. Titze (Singular, San Diego, 1993), pp. 93-142], which uses a more realistic, aeroacoustic model of the vocal tract than classic electrical-analog representations. It accommodates area and hydraulic radius profiles, smooth and abrupt area changes, incorporating end corrections, side branches, and net fluid flows, including turbulence losses incurred through jet formation. Originally, VOAC was tested by comparing vowel formant frequencies (i) uttered by subjects, (ii) predicted using classic electrical analog, and (iii) predicted by VOAC. In this study, VOAC is further validated by comparing the predicted frequency response functions for a range of flow rates with measurements of the radiated sound from a series of mechanical models of unvoiced fricatives [C. Shadle, Ph.D. thesis, MIT-RLE Tech. Report 506 (1985)]. Results show VOAC is more accurate in predicting the complete spectrum at a range of flow rates. Finally, preliminary work is presented with VOAC used to simulate the sound generated at a sequence of stages during the release of a plosive.
3aSCb10. Vocal microtremor assessed by complex demodulation. Eugen Gallasch (Dept. of Physiol., Karl-Franzens Universität, Harrachgasse 21, 8010 Graz, Austria)

Vocal microtremor refers to small involuntary fluctuations of fundamental frequency $\left(f_{0}\right)$ and sound intensity during attempted constant phonation. Physically such an utterance may be considered as a FM/AM process $x(t)$ with $f_{0}$ as carrier frequency (after filtering the acoustic signal around $f_{0}$ to eliminate its harmonics): $x(t)=a(t)^{*} \cos \left(f_{0} t+\phi(t)\right)$ and $f(t)=d[\phi(t)] / d t$. To study the instantaneous changes of $f(t)$ and $a(t)$ as well as their mutual relationships, complex demodulation was applied. First, the complex analytic signal $y(t)$ was formed using the Hilbert operator $\mathbf{H}: \quad y(t)=x(t)+j \mathbf{H}[x(t)]=a(t) * \mathbf{e}^{j\left[f_{0} t+\phi(t)\right]}$. Then demodulation was performed by multiplying $y(t)$ with the negative complex carrier, thus shifting the narrow-band spectral content around $f_{0}$ to zero: $z(t)$ $=y(t) * \mathbf{e}^{-j f_{0} t}=a(t) * \mathbf{e}^{j \phi(t)}$. At least the instantaneous quantities $a(t)$ and $f(t)$ could be recovered from $z(t)$. The analysis was implemented with MATLAB functions including procedures to estimate modulation indices and the cross-correlation coefficient of the demodulated signals. Both waveforms showed periodic as well as irregular patterns and were positively correlated (about 0.5 ), indicating physiological or mechanical coupling in the sound generating mechanisms. This analysis might be further useful to study musical vibrato as well as laryngeal and neurological disorders.

\section{2:00}

3aSCb11. Bifurcations at voice onset-offset. Jorge C. Lucero (Dept. Matematica, Univ. Brasilia, Brasilia DF 70910-900, Brazil, lucero@mat.unb.br)

Oscillation hysteresis is a common phenomenon in cases of flowinduced oscillation. In phonation, it is usually observed as different biomechanical configurations of the vocal fold system between voice onset and offset. From the perspective of the theory of nonlinear dynamical systems, the phenomenon may be described as a combination of a subcritical Hopf bifurcation at which an unstable limit cycle is generated from an equilibrium position of the vocal folds, and a saddle-node bifurcation between limit cycles at which the unstable limit cycle and a stable limit cycle coalesce and cancel each other [J. C. Lucero, J. Sound Vib. (in press)]. This description is investigated using a two-mass model of the vocal folds coupled to a two-tube approximation of the vocal tract. The purpose is to simulate the hysteresis in terms of voice output parameters as airflow, intensity, and fundamental frequency. The simulations show good agreement with available experimental measurements at voice onsetoffset [e.g., L. L. Koenig and R. S. McGowan, J. Acoust. Soc. Am. 100, 2689(A) (1996)]. Bifurcation diagrams are derived to illustrate and discuss the results. [Work supported by the CNPq (Brazil).] 


\title{
Session 3aSP
}

\section{Signal Processing in Acoustics and Engineering Acoustics: Binaural Technology I}

\author{
Mahlon Burkhard, Cochair \\ Sonic Perceptions, Inc., 6964 Kensington Road, Brighton, Michigan 48116, USA \\ Jens Blauert, Cochair \\ Communication Acoustics, Ruhr-Universität Bochum, D-44780 Bochum, Germany
}

Chair's Introduction-7:55

Invited Papers

\begin{abstract}
8:00
3aSP1. Sound localization with unilaterally degraded spectral cues. Frederic L. Wightman and Doris J. Kistler (Waisman Ctr., Univ. of Wisconsin, 1500 Highland Ave., Madison, WI 53705, Wightman@waisman.wisc.edu)

Two cues are thought to determine the apparent position of a sound, interaural time differences and the spectral patterning produced by the pinnae and upper body. The role of the spectral cues is the least well understood. One area of uncertainty is the extent to which cues from both ears are used. This study of the issue asked listeners to indicate the apparent positions of virtual sources synthesized from each listener's own head-related transfer functions (HRTFs). Four types of stimuli were intermingled during a run: (1) all cues preserved, (2) spectral cues degraded at the ear on the side contralateral to the source, (3) degraded cues on the same side as the source, and (4) cues degraded binaurally. Cues were degraded either by smoothing, which preserved the major interaural level cues, or by replacing the natural HRTF with a constant average spectrum, which perturbed the natural interaural level cues. The results suggest that degrading contralateral cues has only a minor effect, while degrading either ipsilateral or binaural spectral cues causes significant localization errors. Results from the two degradation conditions were the same, suggesting that interaural level differences are relatively unimportant localization cues. [Work supported by NIH, NASA, and ONR.]
\end{abstract}

\section{8:20}

3aSP2. Physical modeling of individual head-related transfer functions (HRTFs). Roland Sottek and Klaus Genuit (HEAD acoustics GmbH, Ebertstrae 30a, 52134 Herzogenrath, Germany, Roland.Sottek@head-acoustics.de)

A model of HRTFs was developed based on the Ph.D. thesis of Genuit. The two basic ideas are (1) HRTFs can be described by the influence of a few acoustically relevant objects; first the head was modeled by a sphere and the pinna with cavum conchae by two elliptical disks with an eccentric cylindrical cave (ear canal). (2) Individual variations of HRTFs correspond to variations of geometrical parameters (diameter of sphere, dimensions of disks and cave, ...); the position of the ear canal entrance point is the most important parameter. Simulation results were compared to HRTFs measured with an artificial head of well-known geometry. In general, a good correspondence between calculated and measured HRTFs was found. The correlation is significantly higher if the influence of shoulder/torso is considered, too, modeled by an additional rigid sphere. Second, the shape of head and shoulder/torso was approximated with oblate respective prolate spheroids in order to get even better results. Sound field calculation were performed using numerical methods (boundary element method, source simulation technique) and analytical methods (solution of the wave equation, use of Huygens' integral formula). The interaction between the different reflecting and diffracting bodies was analyzed to validate a simplified model.

3aSP3. The relevance of the shoulder bounce to HRTFs considering head movements. Renato Pellegrini (Inst. für Kommunikationsakustik, Ruhr Univ. Bochum, Universitätsstr. 150, 44780 Bochum, Germany, pelleg@ika.ruhr-uni-bochum.de)

When measuring HRTF data for use in virtual auditory environments, individual HRTFs of subjects are commonly measured keeping the head in the front position vertical to the upper body. This measured data set is then used to simulate the direction of incidence of virtual sound sources. In real world situations, subjects normally tend to turn their heads only, instead of rotating with the whole upper body when focusing on a sound source. In virtual auditory environments this relative head-to-upper-body rotation is not accurately simulated. This error could theoretically deteriorate the perception of virtual sound sources. HRTF data sets were measured for different shoulder to head positions other than front, and psychoacoustical tests were made to give evidence to the importance of shoulder to head positioning. Timbral aspects and localization accuracy in static and dynamic situations were investigated by auditory testing. Results are discussed. 
3aSP4. Determination of optimal HRTFs for binaural synthesis. Flemming Christensen, Henrik Moeller, Soeren K. Olesen, and Pauli Minnaar (Aalborg Univ., Fredrik Bajersvej 7B4, DK-9220 Aalborg OE, Denmark, acoustics@kom.auc.dk)

Earlier studies have shown that a carefully selected human substituting an artificial head can minimize the amount of localization errors for a group of people in a listening experiment, thus indicating that a set of head-related transfer functions (HRTFs) can be found, which to some extent fits a population. This study aims at exploring ways of designing such general sets of HRTFs suitable for larger populations. An effort is put into considering the importance of, e.g., different frequency regions of the transfer functions in order to focus on the most general characteristics and avoid focusing attention on highly individual features. The physical origin of the different parts of the HRTFs will be taken into consideration, and HRTF design methods using parameters derived through signal analysis will be studied.

3aSP5. The effects of source characteristics on the auditory localization of nearby sources. Douglas S. Brungart (Air Force Res. Lab., 2610 Seventh St., Wright-Patterson AFB, OH 45433-7901, brungart@falcon.al.wpafb.af.mil) and Nathaniel I. Durlach (MIT, Cambridge, MA 02139)

A series of recent experiments has examined the auditory localization of a nearby $(<1 \mathrm{~m})$ sound source under four conditions: (1) a fixed-amplitude condition where loudness-based distance cues were available; (2) a monaural condition where the contralateral ear was occluded by an ear-plug and muff; (3) a high-pass condition where the stimulus bandwidth was $3 \mathrm{~Hz}-15 \mathrm{kHz}$; (4) a low-pass condition where the stimulus bandwidth was $200 \mathrm{~Hz}-3 \mathrm{kHz}$. The results of these experiments were compared to those of a previous experiment that measured localization performance for a nearby broadband, random-amplitude source [Brungart et al., J. Acoust. Soc. Am. 102, 3140(A) (1997)]. Directional localization performance in each condition was consistent with the results of previous far-field localization experiments. Distance localization accuracy improved slightly in the fixed-amplitude condition, especially near the median plane, but was severely degraded in the monaural condition. Distance accuracy was also found to be highly dependent on the low-frequency energy of the stimulus: in the low-pass condition, distance accuracy was similar to that in the broadband condition, while in the high-pass condition, distance accuracy was significantly reduced. The results suggest that low-frequency interaural intensity differences are the dominant auditory distance cue in the near-field.

\section{9:40}

3aSP6. Role of two ears in upper hemisphere localization. Masayuki Morimoto (Environ. Acoust. Lab., Faculty of Eng., Kobe Univ., Nada, Kobe, 657-8501 Japan, mrmt@kobe-u.ac.jp)

It is well known that the elevation of a sound source is determined by spectral cues in the median plane, where the input signals to both ears are regarded as being identical. It is, however, still a problem how the elevation of a sound source is determined in any plane apart from the median plane, because the input signals to both ears are not identical. This paper investigates how the input signals to the left and right ears contribute to determine the elevation of a sound source at any position in the upper hemisphere. The localization tests regarding the elevation in five planes parallel to the median plane were performed. In the localization tests, pinna cavities of both or one of two ears were occluded. The results indicate that both ears play a role in determining the elevation of a sound source in any plane. Furthermore, they infer that the summation of spectral features of input signals to the left and right ears contributes to determine the elevation.

\section{0:00-10:20 Break}

\section{0:20}

3aSP7. Generation of binaural information using cross ambiguity functions. Michiaki Uchiyama and Mikio Tohyama (Kogakuin Univ., Bldg. 5-603, Nakano-machi 2665-1, Hachioji-shi, Tokyo, 192-0015 Japan)

This paper describes a method to generate binaural information. First of all, the accuracy of a conventional linear interpolation was examined by using signal-to-deviation ratio (SDR) and spectrum distortion. It was found that the accuracy of left and right direction HRTFs $\left(60-120^{\circ}\right)$ did not deteriorate despite the presence of a wide directional interval (SDR $\cong 23 \mathrm{~dB}$ ). However, the accuracy of front and back direction HRTFs $\left(0--60^{\circ}\right.$ and $\left.120-180^{\circ}\right)$ deteriorated as the interval broadened. For the shade-side ear's HRTFs, particularly, it was confirmed that the accuracy of linear interpolation deteriorated dramatically ( $\mathrm{SDR} \cong 8 \mathrm{~dB})$. Therefore, a method was investigated for generating a shade-side ear's HRTF by using cross ambiguity functions (CAFs) whose model is related to dichotic listening. A shade-side HRTF could be generated with about a 10-dB SDR from a sunny-side HRTF, while the SDR becomes about $8 \mathrm{~dB}$ for a conventional linear interpolation estimation method. In this paper, the independence of HRTFs is also discussed, using the singular value decomposition from the point of view that it is possible to generate a new vector from a linear combination of vectors. In this article the accuracy of a linear interpolation from a base of HRTFs by SDR and spectrum distortion is examined.

\section{0:40}

3aSP8. Audition and the sense of presence in virtual environments. R. H. Gilkey, B. D. Simpson, S. K. Isabelle, A. J. Kordik (Dept. of Psych., Wright State Univ., Dayton, OH 45435), and J. M. Weisenberger (Ohio State Univ., Columbus, OH 43210)

Historical reports from suddenly deafened adults describing a sense of detachment from a world devoid of auditory input suggest that auditory cues may be crucial for achieving a sense of presence in virtual environments [R. H. Gilkey and J. M. Weisenberger, Presence 4, 357-363 (1995)]. However, factors other than acoustic fidelity can affect the perceived quality of a virtual auditory display. For example, Simpson et al. [J. Acoust. Soc. Am. 100, 2633(A) (1996)] showed that the sense of presence in virtual auditory environments was driven by characteristics of the real listening environment in which the virtual audio was heard. Specifically, when 
the virtual auditory environment matches the real listening environment, the listening experience is particularly compelling. However, it was not clear whether auditory or visual experience with the real room mediated this effect. This paper describes attempts to separate the impact of auditory and visual information on presence by systematically manipulating these cues in synthesized environments. Implications for design of virtual environments and spatialized auditory displays are considered. [Work supported by AFOSR and Ohio Board of Regents.]

\section{1:00}

3aSP9. A series of experiments exploring the spatial separation of multiple talkers with 3-D audio technology. Richard L. McKinley, Mark A. Ericson, W. Todd Nelson (Air Force Res. Lab., 2610 Seventh St., Wright-Patterson AFB, OH 45433, rlmckinley@falcon.al.wpafb.af.mil), Robert S. Bolia (Veridian, Dayton, OH 45431), and Brian D. Simpson (Wright State Univ., Dayton, OH 45435)

Most auditory displays are monaural and do not take advantage of the capacity of the binaural system for spatial separation of multiple simultaneous talkers. A program of research has been developed at the Air Force Research Laboratory to investigate the enhanced communication effectiveness obtained with 3-D audio displays. Factors that are being manipulated include angular separation, the number of competing messages, and the sex of the target talker. Angular separation has been studied in both the horizontal and median planes, with between two and eight competing messages. The effect of duration of temporal overlap has also been explored. The goal of this research is to discover the maximum number of speech channels that a listener can effectively monitor, and whether this number is augmented by 3-D audio technology. Potential applications of 3-D audio technology for communications separation will be discussed.

\section{1:20}

3aSP10. Sound-image broadening by a single echo and its relation to the transient interaural cross-correlation function. Hirofumi Yanagawa (Chiba Inst. of Technol., 2-17-1 Tsudanuma, Narashino-shi, Chiba-ken 275-0016, Japan) and Takatoshi Okuno (Kogakuin Univ., Tokyo 192-0015, Japan)

The sounds reproduced by two loudspeakers often consist of a preceding sound and its echo when the listener's position is offset like the position of a driver when listening to a car stereo. Consequently, the sound image is broadened by the echo. In this article, the width of the sound image produced by the preceding sound and the echo under a loudness balance condition were examined. TRICC (the maximum value of the transient interaural cross-correlation function) was then applied to analyze such sound image broadening. In hearing experiments, a pair of noise bursts was used as a model of transient sound, such as speeches and musical sounds. A pointer noise (a pair of noises with correlation coefficients) was used to express the width of the sound image. The experiments showed that the width of the sound image increases as delay time between the preceding sound and the echo increases. When the delay time becomes longer than $10 \mathrm{~ms}$, the width of the sound image corresponds to the value of the interaural correlation coefficients and not to the value of TRICC or IACC.

\section{1:40}

3aSP11. Applications of virtual auditory space in psychoacoustics and neurophysiology. Klaus Hartung (Inst. of Commun. Acoust., Ruhr Univ., D-44780 Bochum, Germany, hartung@ika.ruhr-uni-bochum.de), Susanne J. Sterbing (Dept. of Zool. a. Neurobiol., Ruhr Univ., D-44780 Bochum, Germany), Clifford H. Keller, and Terry T. Takahashi (Inst. of Neurosci., Univ. of Oregon, Eugene, OR 97403)

Virtual auditory space (VAS) refers to a technology where the source signal is convolved with filters called head-related transfer functions (HRTFs) and presented over headphones to recreate the signal at the eardrum that would have been received from a source in the free-field. VAS is a powerful tool because it allows the simulation of complex and realistic scenarios (e.g., echoes, reverberation, moving sources) that are impossible or too complicated for free-field setups, which often contain distortions caused by the setup itself (e.g., stereotactic bench, headholder). Crucial points of VAS are the measurement, equalization and interpolation of the HRTF. Different methods will be reviewed and the results of VAS and free-field stimulation for humans, guinea pigs, and barn owls will be compared. For barn owls, the amplitude spectra of virtual sounds were within $\pm 1 \mathrm{~dB}$ of amplitude spectra of free-field sounds measured near the eardrum. The neuronal responses to free-field and VAS presentations were nearly indistinguishable. For research with humans and guinea pigs, it was necessary to use the HRTF of each individual, whereas in the barn owl, only minor differences between the neuronal responses to the individual and nonindividual HRTF were observed. [Work supported by DFG, NIDCD, and NATO.]

\section{2:00}

3aSP12. The importance of binaural auralization for automotive industry. Klaus Genuit and Jens Poggenburg (HEAD acoustics GmbH, Ebertstrae 30a, 52134 Herzogenrath, Germany, klaus.genuit@head-acoustics.de)

In the automotive industry reliable information about the acoustical situation of a vehicle is necessary in an early stage of the development process. This enables the involved parties to work effectively on sound design and sound quality tasks. In this context, the so-called "Binaural Hybrid Model" has been developed. It is based on the determination of binaural transfer paths at vehicles using corresponding measurements. They are combined with airborne and structure-borne noise measurements at test rigs. Applied to vehicle interior noise caused by the engine, the influence of modified transfer paths, airborne radiation and/or input accelerations on the acoustical situation at the passenger's position can be simulated binaurally. This includes the consideration of the head orientation and the aurally equivalent reproduction of the interior sound. It enables the engineer to judge the resulting sound quality before a prototype is realized. The presented model considers airborne shares up to $12 \mathrm{kHz}$ and structure-borne ones up to $2 \mathrm{kHz}$. An application for different vehicles with the same engine clarifies in which way relevant causes for customer complaints at one vehicle may be determined. The variation of single transfer paths and the resulting interior noise will be included. 


\title{
Session 3aUWa
}

\section{Underwater Acoustics: Scattering from Finite Structures Near Boundaries}

\author{
John A. Fawcett, Cochair \\ Defence Research Establishment Atlantic, 9 Grove Street, P.O. Box 1012, Dartmouth, Nova Scotia B2Y 3Z7, Canada \\ Hans Georg Schneider, Cochair \\ Forschungesanstalt der Bundeswehr für Wasserschall und Geophysik, Klausdorfer Weg 2-24, D-24148 Kiel, Germany
}

\section{Invited Papers}

8:40

3aUWa1. Modeling scattering by objects near an interface. John A. Fawcett (Defence Res. Establishment Atlantic, P.O. Box 1012, Dartmouth, NS B2Y 3Z7, Canada)

For an object totally above or below an interface, the overall scattering from the object and the interface can be modeled as a series of multiple interactions [e.g., Hackman and Sammelmann, J. Acoust. Soc. Am. 84, 1813-1825 (1988)]. The theory is outlined for a spherical or infinite cylindrical shell and the effects of the sediment sound speed and the source/receiver geometry on the backscattered time series are numerically investigated. The case of an object in a rough or range-dependent waveguide is also considered. Partial burial of an object is discussed. Some comparisons of modeled and experimental data (both in the spectral and temporal domains) are presented.

\section{9:00}

3aUWa2. Measurement of 3-D scattering from buried targets in very shallow water. Henrik Schmidt (MIT, Cambridge, MA 02139), Alain Maguer, Edoardo Bovio, and Warren Fox (SACLANT Undersea Res. Ctr., I-19138 La Spezia, Italy)

The objective of the GOATS'98 experiment was to determine the three-dimensional, spatial characteristics of seabed target scattering and reverberation in coastal environments with water depth less than $15 \mathrm{~m}$. The experiment was carried out at Marciana Marina, Elba Island, in May 1998 in a joint effort between SACLANTCEN and MIT. Artificial targets were buried at various depths into the sandy bottom and insonified by a parametric source mounted on a 10-m tall tower which could be repositioned on a bottom-mounted rail under remote control. This arrangement allowed for insonifying the targets at a wide range of incident angles, including both the sub- and supercritical regimes. The scattering and reverberation were measured using a suite of fixed and mobile arrays. Specifically, an eight-element line array was mounted on an MIT Odyssey-II autonomous underwater vehicle (AUV) in a "swordfish" configuration. The AUV was launched from R/V ALLIANCE, anchored off-shore, to perform survey patterns in the target area, way-point navigated using a long baseline acoustic navigation network. During these surveys the parametric source would insonify the seabed at a high repetition rate, allowing for dense mapping of the 3-D scattering and reverberation. This paper reviews the experiment and the results, with particular emphasis on the versatility of using the new AUV technology for shallow-water target scattering measurements.

3aUWa3. Acoustic investigation of objects in shallow water using FEM and BEM. Otto von Estorff (Ocean Eng. II, TU Hamburg-Harburg, D-21071 Hamburg, Germany, estorff@tu-harburg.de)

The acoustic behavior of a submerged object is strongly influenced by the existence of reflecting boundaries, namely the water surface or the bottom. Only in some rare cases can it be assumed that the water region is "infinite." The present paper is related to the investigation of underwater objects in shallow water by means of numerical methods. These must be able to take into account fluid/structure interaction effects as well as different boundary conditions. In the suggested methodology, displacement-based finite elements for the structure are coupled to acoustic boundary elements for the fluid medium. An underwater plate and a submerged cylinder will be investigated in different parametric studies. It will be shown how the boundaries (surface, bottom) can be taken into account, and in which cases the effect of boundaries could be neglected. Moreover, the required computational resources as well as the applicability of different methods will be discussed. 
3aUWa4. A comparison of benchmark acoustic scattering solutions with tank experiments on buried and partially buried spheres. Raymond Lim (Coastal Systems Station, Dahlgren Div./Naval Surface Warfare Ctr., Code R22, 6703 W. Hwy. 98, Panama City, FL 32407-7001, lim@atcf.ncsc.navy.mil) and Harry J. Simpson (Naval Res. Lab., Washington, DC 20375-5350)

Predicting the scattering response of objects near boundaries is of growing interest in underwater acoustics, especially when these objects are buried. Standard propagation models suggest that burial in fast attenuating ocean bottoms can seriously retard efforts to find such objects with high-frequency imaging sonar because of the need to operate at close range to overcome loss in the backscattered signal strength, resulting in low search rates. However, recent observations of anomalous sound transmission at shallow grazing angles as well as anomalous long-range detections of buried objects call into question standard assumptions made in regard to the modeling of sound transmission through the water/sediment interface. The present work investigates the breakdown of these assumptions for the scattering problem by comparing laboratory measurements conducted in the NRL Shallow Water Laboratory with predictions of exact scattering solutions for simple targets at various degrees of burial. Scattering data collected recently for a 60-cm-diam. spherical steel shell is used in the investigation. A discussion of the agreement between theory and measurements, features of special interest, and the consequences to our understanding of the acoustics of buried objects is given. [Work supported by ONR.]

10:00

3aUWa5. Modeling scattering from objects near boundaries using infinite elements. David Burnett (SACLANT Undersea Res. Ctr., Viale San Bartolomeo 400, 19138 San Bartolomeo, La Spezia, Italy, burnett@saclantc.nato.int) and Richard Holford (Lucent Technologies, Bell Labs, Denville, NJ 07834)

There has been a rapidly growing interest in using infinite elements for modeling acoustic scattering and radiation from finite objects in unbounded regions. The paper will briefly review current infinite element technology, which is based on radial expansions in curvilinear coordinate systems. Although the radial expansions are derived for free-space conditions (4 pi steradians), the elements are also valid for half-spaces, using local hard, soft, or finite impedance conditions on the boundary. In this approach, wave propagation is modeled in only one half-space, with the boundary conditions on the interface approximating the effects of the other half-space, thereby enabling these types of infinite elements to model scattering from objects near, and on the illuminated side of, the boundary of a half-space. Theory and numerical results will be presented. A more general approach, which models wave propagation in both half-spaces, uses infinite elements in both half-spaces, with appropriate continuity conditions at the interface. This is the approach currently under investigation by the authors; their findings will conclude the paper.

10:20-10:40 Break

Contributed Papers

10:40

3aUWa6. Measurements of acoustic scattering from partially and completely buried thin-shelled targets. Warren L. J. Fox, Alain Maguer, Alessandra Tesei (NATO SACLANT Undersea Res. Ctr., Viale S. Bartolomeo 400, 19138 La Spezia, Italy), and Henrik Schmidt (MIT, Cambridge, MA 02139)

Conventional high-frequency imaging sonars become less effective in detecting and classifying bottom-laid targets when the targets become buried into seabottom sediments. Some recent work at the NATO SACLANT Undersea Research Centre has concentrated on investigating the use of lower-frequency sonars $(2-16 \mathrm{kHz})$ in order to better exploit scattering features of buried targets that can aid in their detection and classification. Part of the recent GOATS'98 experiment performed near Elba Island, Italy, involved carefully controlled monostatic measurements of scattering by thin-shelled objects (spheres and cylinders) that were partially and completely buried in sand, as well as in free-field. Preliminary results are presented which show promising comparisons of the data to existing models. In addition to examining peak levels of the returns for detection purposes, special attention is paid to resonance effects (examined in the time and frequency domain), and the effect of burial on this part of the target response. Scholte-Stoneley and Lamb-type waves can be clearly identified in the backscattered signals. The issue of inverse scattering for classification purposes is also addressed. [Work supported in part by EC MAST Programme and ONR.]

\section{1:00}

3aUWa7. Target classification in shallow waters by time-frequency filtering. Nicole Gache and Patrick Chevret (CPE Lyon, LASSSO (LISA, EP92 CNRS), 43 Bd. du 11 Novembre 1918, BP 2077, Bat. 308, F 69616, Villeurbanne Cedex, France)

Efficient sonar target recognition must be based on a set of relevant parameters extracted from the echoes and directly related to the geometrical and mechanical properties of the target. Previous studies have shown that a wideband approach based either on simple spectral modeling or on time-frequency analysis is particularly suited to the understanding of echo formation mechanisms and to the classification in the free field (recognition rate as high as 96\%). In shallow waters, the influence of the boundaries considerably reduces the performance of these methods due to the presence of several spurious echoes superimposed to the target echo components. Classification performance can be improved by using a method based on time-frequency filtering matched to a free-field reference target response. This method will be described and applied to experimental data issued from tank experiments. Several typical configurations have been first investigated: free-field, semi-infinite space for a set of targets (shells and solid targets of different shapes). It gives more than encouraging results, especially for the recognition of a spherical shell (about $100 \%$ of recognition). The method is now extended to a shallow-water configuration including seabed interaction. 
$11: 20$

3aUWa8. Numerical simulation of transient scattering from bodies and shells in a layered fluid-solid medium. Ilkka Karasalo (Dept. of Underwater Wave Propagation and Active Sonar, FOA, Stockholm, Sweden) and Johan Mattsson (Royal Inst. of Technol., Stockholm, Sweden)

Results from numerical simulation of transient scattering by 3-D objects immersed in seawater or buried in the bottom sediment are presented, and compared with data from a shallow-water experiment in the Baltic in which a bottom-penetrating parametric sonar was used for detection of submerged or buried objects. A range-independent fluid-solid model of the medium is used. The scatterers have smooth shape and are modeled as acoustically impenetrable or alternatively penetrable fluid-enclosed thin elastic shells. The dynamics of such shell-structured scatterers are described by Love's equations for thin shells. A recently developed numerical technique, based on a frequency-domain boundary integral equation (BIE) formulation of the scattering problem, is used to compute the scattered field. The Green's function of the layered medium is obtained by an adaptive transform integral method. The BIE is discretized by high-order B-splines, point collocation, and global numerical quadrature. The incident field may be excited by a point source, a point source array, or a plane wave. Scattering of transient fields is computed by Fourier synthesis of monofrequent field components. The composite simulation being highly parallelizable, a version for execution on workstation clusters under MPICH has been implemented, allowing for convenient handling of also computationally demanding broadband excitations.

\section{1:40}

3aUWa9. Sea experimental detection of an array of hollow spheres. R. Carbó and A. Molero (Instituto de Acústica, CSIC, Serrano 144, 28006 Madrid, Spain, iaccf31@fresno.csic.es)

A study of acoustic backscattering produced by a system of scatter arrays is reported. A simplified theoretical model has been developed to study the interference phenomenon due to the echo formation. This model establishes the relation between system geometry (target dimensions, scat- ter distance and depth) and sonar performances (directivity, pulse length, pulse repetition rate, wave length, and ship speed) getting a better system response. Target strength of a single scatter was defined at the laboratory, at an underwater tank with a dual-frequency side scan sonar working at $100 \mathrm{kHz}$. Different array geometries were placed in order to characterize minimum distance between scatters needed to discriminate them at the echogram. Experimental test has been realized at the sea with the same side scan sonar at $100-$ and $500-\mathrm{kHz}$ frequency. Scatter arrays were placed at $15-\mathrm{m}$ depth, were clearly located, and identified from a distance of 50 $\mathrm{m}$. To identify the system two different arrays were placed: a short one with a reduced number of scatters, and a second one with a large amount of same scatters, with a total length of $48 \mathrm{~m}$.

\section{2:00}

3aUWa10. Sound diffraction by an underwater topographical ridge. Ji-Xun Zhou, Xue-Zhen Zhang, and Yun S. Chase (School of Mech. Eng., Georgia Inst. of Technol., Atlanta, GA 30332-0405)

Scale model experiments have been conducted to study the diffraction of sound by an underwater convex surface with a very soft boundary (closed-cell foam material). The diffraction boundary can be well described as a locally reacting surface based on measured acoustic parameters (very low speed and density, and very high attenuation). The diffraction acoustic pressure (insertion loss) behind the ridge and along the diffraction surface were measured. Measured insertion losses were compared with the theory of matched asymptotic expansion (MAE), developed by Fock for electromagnetic waves and Pierce for acoustic waves. The experimental results are in good agreement with predictions from the MAE theory. This technique might be used to infer acoustic parameters for materials. It was found that the model of Delany and Bazley, which is widely used in architecture acoustics and outdoor sound propagation, may be inappropriate for the description of an underwater locally reacting impedance. [Work supported by ONR.]

\section{Contributed Posters}

These posters will be on display in the Poster Gallery from Thursday to Friday, 18-19 March. Authors will be at their posters from 10:00 a.m. to 12:00 noon on Wednesday, 17 March.

3aUWa11. Analysis of boundaries' effects in the evaluation of transient ultrasonic fields scattered by a rigid reflector. Khelladi Hassina and Djelouah Hakim (USTHB, Inst. d'Electron., Departement Instrumentation, BP32 El Alia 16111, Algeria)

One approach used to estimate the transient field scattered by a target with a known dimension and geometry is the convolution method. In the hypothesis of linear acoustic, and for a perfectly rigid reflector immersed in an isotropic, homogeneous, and lossless fluid, the detected acoustic pressure is expressed in the form of a time convolution between the radiator velocity profile and the transmit-receive impulse response. This last depends on the geometry of the transducer, the target, their relative position, and physical characteristics of the propagation medium. For that purpose, some simulations were done. The pressure waveform is explained in terms of the plane and edge waves. In order to determine the boundaries' effects on the detected pressure, targets of different size were used. The obtained results show that the edge waves were insignificant when large size targets are used.

3aUWa12. Sound scattering by moving inhomogeneity. Andrew G. Semenov and Vadim N. Alexeyev (N. N. Andreev Acoust. Inst., Russian Acad. of Sci., 4 Shvernik St., 117036 Moscow, Russia, bvp@acoins.msk.ru)

This report considers the propagation of sound in the vicinity of a rather general class of localized flows induced by the motion of spherically symmetric structures of the type of solid spheres and droplets or vortices in the liquid, say, ocean environment. Acoustic scattering from 
such inhomogeneities, situated inside various flows, is analyzed with the aid of the M. J. Lighthill equation boundary value problem solution-both in the presence of vorticity and without it. The role of a moving body generating inhomogeneity as well as the role of the surface at which the tangential component of flow velocity could suffer a discontinuity are demonstrated in a most general form. Comparison of body and flow con- tribution to the observed scattered field is presented. The physical condition of flow presumably yields in a forward scattering direction-a situation of the type encountered in a conventional ocean tomography system, comprising a source-detector line, is proposed. The angular structure of the scattering amplitude and the frequency dependence of the scattered sound are evaluated.

WEDNESDAY MORNING, 17 MARCH 1999

ROOM H2053, 9:00 A.M. TO 12:00 NOON

\title{
Session 3aUWb
}

\section{Underwater Acoustics and Acoustical Oceanography: Ultra Low Frequency $(<1 \mathrm{~Hz})$ Ocean Acoustics}

\author{
Ralph A. Stephen, Cochair \\ Woods Hole Oceanographic Institute, Woods Hole, Massachusetts 02543, USA \\ Jean Paul Montagner, Cochair \\ Department Sismologie, Institut de Physique du Globe de Paris, 4 Place Jussieu, 75232 Paris 05, France
}

\section{Invited Papers}

\begin{abstract}
9:00
3aUWb1. A review of ocean acoustics in the band 0.001-1.0 Hz. Ralph A. Stephen (Woods Hole Oceanogr. Inst., Woods Hole, MA 02543, rstephen@whoi.edu)

The first ultra-low frequency (ULF) (0.001-1 Hz) ambient acoustic noise measurements were made in the 1960s using the Columbia-Point Arena ocean-bottom seismic station. Interest in ULF acoustics was spurred in the mid-1980s by the development of the differential pressure gauge. Also in the mid-1980s the development of high dynamic range, broadband seismometers revolutionized whole earth seismometry. The Global Seismic Network (GSN) set as its goal the uniform distribution of 128 seismic stations over the surface of the globe, to provide high-quality data sets for whole earth tomography and other earth scale problems. To accomplish this goal requires about 20 permanent stations on the deep ocean floor. In the future, the acquisition of long time series of ULF acoustic noise in the deep ocean will become much more convenient and the opportunity will be given to exploit these data sets to study oceanographic and earth processes. The sources of ambient noise in this band include infra-gravity waves, swell interaction at coastlines, currents and turbulence near the seafloor, earthquakes, tsunamis, nonlinear interaction between ocean-surface waves, and air turbulence. Water column reverberations, Stoneley modes in sediment layers, and the effects of propagation from distant sources also modify the pressure spectra. [Work supported by NSF.]
\end{abstract}

\section{9:20}

3aUWb2. Ocean bottom seismic noise (1-10 000 s). Jean-Paul Montagner (Dept. Sismologie, IPG, 4 Pl. Jussieu, 75252 Paris, France, jepm@ccr.jussieu.fr)

There is now a good consensus in the geophysical scientific community of the necessity to install broadband seismic stations on the ocean bottom in order to improve our knowledge of the deep Earth dynamics. However, it is necessary to first investigate the level of seismic noise (0.01-10000 s) and to compare it with the expected amplitude of seismic signal due to earthquakes. During the French pilot experiment SISMOBS/OFM (28 April-11 May 1992) in the Atlantic Ocean, two sets of Guralp CMG3 broadband seismometers were successfully installed and recovered. The first set, named OFP, was installed inside the ODP borehole 396B at 292 $\mathrm{m}$ below seafloor and the second one, named OFM, was installed on the sea bottom (semi-buried) close to the hole. A comparison of broadband seismic noise (1-3600 s) between OFP, OFM, and a typical continental GEOSCOPE seismic station SSB shows that the noise level of OFM is smaller (resp. larger) at periods longer than $20 \mathrm{~s}$ (resp. shorter than $2 \mathrm{~s}$ ) than the one in the borehole and in SSB. The detection threshold is very low and makes it possible to get good records of teleseismic events as small as 5.4. These results were very encouraging since they demonstrate that in the future ocean bottom seismometers will provide very valuable information on earthquakes and Earth structure.

\section{9:40}

3aUWb3. Lessons from the Ocean Seismic Network Pilot Experiment. John A. Collins, Ken R. Peal, Ralph A. Stephen (Woods Hole Oceanogr. Inst., Woods Hole, MA 02543, jcollins@whoi.edu), Frank L. Vernon, John A. Orcutt, John A. Hildebrand, and Fred N. Spiess (Scripps Inst. of Oceanogr., La Jolla, CA 92093)

The basic goal of the Ocean Seismic Network Pilot Experiment (OSNPE) was to learn how to make reliable broadband (0.001-10 $\mathrm{Hz}$ ) seismic measurements in the deep ocean. This objective addresses both the requirement for long-term, observatory-based measurements at a small number of carefully selected sites (i.e., the Ocean Seismic Network), and for portable arrays of broadband seismographs that can be deployed for about one year to study specific geological features. The OSNPE ran from February to June 1998. The site of the experiment was ODP Hole 843B (site OSN-1) located about $225 \mathrm{~km}$ southwest of Oahu, Hawaii. At the OSN-1 
site, three broadband seismographs were deployed within $300 \mathrm{~m}$ of each other. The seismometers were (i) a Teledyne GeoTech KS54000 deployed in a borehole $240 \mathrm{~m}$ beneath the seafloor; (ii) a Guralp CMG-3T deployed on the seafloor; and (iii) a Guralp CMG-3T buried just beneath the seafloor. The latter two systems also carried long-period pressure sensors. Meteorological and oceanographic data were also recorded continuously throughout the experiment. The magnitude and direction of seafloor currents were recorded at the experiment site itself. Four NOAA/NDBC buoys in the vicinity of OSN-1 recorded wind speed, wave-height, and wave-direction. [Work supported by NSF.]

10:00

3aUWb4. Evaluation of teleseismic waveforms and detection thresholds from the OSN pilot experiment. Frank L. Vernon, John A. Orcutt, Gabi Laske, Fred N. Spiess (Scripps Inst. of Oceanogr., La Jolla, CA 92093, flvernon@ucsd.edu), John A. Collins, Ken R. Peal, Ralph A. Stephen, and Cecily J. Wolfe (Woods Hole Oceanogr. Inst., Woods Hole, MA 02543)

The first Ocean Seismic Network Pilot Experiment (OSNPE) took place from January to June of 1998. The site of the experiment was located about $225 \mathrm{~km}$ southwest of Oahu, Hawaii, where three broadband seismographs were deployed within $300 \mathrm{~m}$ of each other, (i) deployed in a borehole 240-m beneath the seafloor; (ii) on the seafloor; (iii) buried just beneath the seafloor. Each of the seafloor broadband seismograph stations also carried a Cox-Webb long-period, differential pressure gauge (DPG). The OSNPE ran concurrently with PELENET, a temporary deployment of five island broadband stations and SWELL, a temporary deployment of eight DPGs. Over 50 teleseismic earthquakes were observed. The broadband seismograms recorded during the OSNPE are coherent in the frequency band $0.01-0.1 \mathrm{~Hz}$. At higher frequencies $(0.1-3 \mathrm{~Hz})$, there is considerably more variation between the borehole, buried, seafloor, and island sites. Careful examination of the waveforms shows significant differences in the frequencies above $0.1 \mathrm{~Hz}$ between the buried/surface broadband ocean bottom seismographs and the borehole seismograph. These variations appear to be associated with shear-wave reverberations and/or Stonely wave propagation within the sediment section. The teleseismic earthquakes permit a preliminary evaluation of the long period $(0.01-0.07 \mathrm{~Hz})$ detection thresholds.

10:20-10:40 Break

10:40

3aUWb5. GEOSTAR benthic observatory: Technological results. Hans W. Gerber (Technische Fachhochschule Berlin, Luxemburger Str., 10 D-13353 Berlin, Germany, hwgerber@tfh-berlin.de), Paolo Favali, Giuseppe Smriglio (Istituto Nazionale di Geofisica, 60500143 Roma, Italy), Francesco Gasparoni (Tecnomare SpA, San Marco 358430124 Venezia, Italy), Jean Marvaldi, and Jean-Michel Coudeville (IFREMER, Plouzane, Brest, France)

GEOSTAR (GEophysical and Oceanographic STation for Abyssal Research) is a project funded by European Commission, MAST III. The aim of the project was the development of the first prototype of an innovative deep-sea benthic observatory capable of carrying out long-term scientific observations at abyssal depths (up to $-4000 \mathrm{~m}$ ). The prototype has been realized and tested successfully in Adriatic Sea in late summer 1998. GEOSTAR is made up of two main subsystems: Bottom Station and Mobile Docker. The Bottom Station includes the acquisition and power systems, all the scientific sensors and hosts the Communications. The Mobile Docker is a dedicated tool for surface-assisted deployment and recovery. GEOSTAR is characterized by a lightweight and modular frame, autonomous mission control capabilities, and multiple possibilities of interfacement with external devices. The Bottom Station is presently equipped with geophysical, geochemical, and oceanographic instruments (a triaxial seismometer, vectorial and scalar magnetometers, CTD, transmissometer, ADCP, and an electrochemical package), but it can easily host other sensors for different experiments. The observatory was deployed in the Adriatic Sea on 13 August 1998 and recovered on 2 September 1998. The observatory worked properly, recording 440 hours of scientific data. Data were processed, elaborated, and interpreted based on a multidisciplinary approach.

\section{Contributed Papers}

11:00

\begin{tabular}{lllllr} 
3aUWb6. Low-frequency & \multicolumn{2}{c}{ dispersion computations } & by \\
compound-matrix & propagation. & Sven Ivansson (FOA & 640 , Natl. \\
Defence Res. & Establishment, $17290 \quad$ Stockholm, & Sweden, \\
sveni@sto.foa.se) & & & &
\end{tabular}

The basic propagating waveguide modes in underwater acoustics have lower cutoff frequencies. As the frequency is decreased below the cutoff frequency, the modal horizontal slowness typically becomes complex and tends to infinity while the modal wave number tends to a nonvanishing complex number. In certain laterally homogeneous fluid-solid media, there also exist slow $P-S V$ modes for which the horizontal slowness tends to infinity along the real axis while the horizontal wave number tends to zero as the frequency tends to zero. The flexural wave for an ice sheet on top of the ocean is a particular example. To investigate the lowfrequency behavior of the modal horizontal slowness for these kinds of modes, computations of dispersion curves by propagator techniques are attempted for media composed of homogeneous fluid and solid layers. However, loss of numerical precision by cancellation effects appears for the needed elements of the solid-layer compound-matrix propagators. Guided by theoretical results for the asymptotic growth of these elements, cancellation-free expressions are derived. The harmful contributions causing loss of numerical precision at low frequencies are eliminated analytically. As an application of the new expressions, a numerical case study is performed. Improved results on low-frequency asymptotic expansions of the modal slownesses are obtained.

11:20

3aUWb7. On comparative contributions of natural ultra-low-frequency hydrodynamic wave fields to sound signals' variability on extended shallow-water traces. Andrew G. Semenov (N. N. Andreev Acoust. Inst., Russian Acad. of Sci., 4 Shvernik St., 117036 Moscow, Russia)

The ocean's ultra-low-frequency natural sound variability mechanism, related to sound interaction with extra slow hydrodynamic natural wave processes, gravitational surface, and internal wave, observed in the range of $\mathrm{rad} / \mathrm{s}$ on long traces, is discussed. In the absence of natural internal waves, the ocean hydrodynamic field here is close to potential, so that its interaction with the sound field is minimal. Estimates are based on the model of ocean hydrodynamic waves representing the refraction index lattice for propagating sound modes. Small angle sound interaction with 
weak enough surface waves lattice leads to additional mode phase transformation increasing with trace length. Interaction is shown to be especially substantial for low-frequency sound (of frequency less than 100$200 \mathrm{~Hz}$ ) and very long traces (of length not less than 80-100 km) in a shallow ocean region, where it could be comparable to interaction observed due to internal waves. While for a given sound frequency value and short trace length, sound-hydrodynamic wave fields' interaction is determined exclusively by lattice refraction factor, for long enough traces, interaction is defined by refraction index and hydrodynamic wavelength product. This parameter variability for surface gravity waves in different ocean regions, based on generalized bottom pressure measurement data, is presented.

\section{1:40}

3aUWb8. Validation and application of an effective medium theory to heterogeneous anisotropic structures. Catherine Stamoulis (Dept. of EAPS, MIT, Cambridge, MA 02139 and Dept. of EPS, Harvard Univ., Cambridge, MA 02138), Dimitri Komatitsch (Harvard Univ., Cambridge, MA 02138), Thomas Jordan (MIT, Cambridge, MA 02139), and Jeroen Tromp (Harvard Univ., Cambridge, MA 02138)
The polarization anisotropy in the upper mantle requires inhomogeneities with small scale lengths. To represent heterogeneous anisotropic structures, such as ocean basins, Jordan et al. (1996) formulated stochastic models for small-scale heterogeneities, as samples of a random field of fourth-order hexagonally symmetric elasticity tensors $C_{i j k l}$. The random field has a characteristic horizontal wave number $\kappa$, independent of azimuth, and a characteristic vertical wave number $\eta \kappa$. The medium is therefore statistically transversely isotropic with horizontal heterogeneity scale $1 / \kappa$, aspect ratio $\eta$, and self-affine scaling at high wave numbers specified by a fractal dimension $D$. The propagation of seismic waves with lengths $\gg 1 / \kappa$ is described by five effective elastic constants, calculated to second order in the scattering by contracting the static fourth-order Green's strain tensor with the eighth-order variance tensor for $C_{i j k l}$. The validity of this self-consistent effective medium theory is tested by comparing the propagation characteristics that it predicts, as a function of range and frequency, with those measured from synthetic seismograms computed for realizations of the medium using a spectral element method [Komatitsch and Vilotte (1998)]. In particular, the theoretical, frequency-independent phase delays are compared with the frequency-dependent phase delays from the synthetic seismograms.

WEDNESDAY MORNING, 17 MARCH 1999

POSTER GALLERY, 8:00 TO 10:00 A.M.

\title{
Session 3aUWc
}

\section{Underwater Acoustics: Transducers, Arrays and Instrumention (Poster Session)}

\author{
Robert W. Farwell, Chair \\ Neptune Sciences, Inc., 150 Cleveland Avenue, Slidell, Louisiana 70458, USA
}

\section{Contributed Papers}

\begin{abstract}
All posters will be on display in the Poster Gallery from Monday to Wednesday, 15-17 March. Authors will be at their posters from
\end{abstract} 8:00 a.m. to 10:00 a.m. on Wednesday, 17 March.

3aUWc1. Optoacoustic technique for remote control of underwater oceanographic instrumentation. Sergey V. Egerev (N. N. Andreev Inst. of Acoust., 4 Shvernika St., 117036, Moscow, Russia) and Andrey K. Morozov (Russian Acad. of Sci., Moscow, Russia)

A sound source excited through the irradiation of a water surface with a powerful laser pulse is called an optoacoustic (OA) source. This phenomenon makes possible the remote control of submerged oceanographic devices (buoys) directly from a flying vehicle equipped with a pulse laser. The principal factors are analyzed (i.e., reverberation, ambient noise, etc.) which influence the efficiency of OA digital data transfer. The gap between the two neighboring laser pulses is the only parameter of signal that allows the transmitting of data through this remote technique. This makes different versions of pulse-position modulation preferable. Algorithms for processing detected signals are described. The optoacoustic communication system was tested at the White Sea. $\mathrm{A} \mathrm{CO}_{2}$ laser on board a research vessel was used to launch an OA source. The acoustic signal level of about $10^{5} \mathrm{~Pa}$ at a $1-\mathrm{m}$ depth was achieved within the frequency band of up to $100 \mathrm{kHz}$. Test data messages at a rate of 2 bits per second for laser pulse repetition rate of about $0.5 \mathrm{~Hz}$ were transmitted along an experimental vessel-to-vessel 480-m line. The OA technique works well for devices submerged to depths of $2000 \mathrm{~m}$ and possibly more. [Work supported by RFBR.]

3aUWc2. Laser optoacoustic diagnostics of sea ice. Alexey $O$. Maksimov (Pacific Oceanological Inst., 43 Baltic St., Vladivostok, 690041, Russia,root\%dan86@ poi.marine.su)

The emphasis of the current study is the analysis of the mechanisms of generation and propagation of optoacoustical (OA) signals in the upper ocean for the winter season or Arctic sea, when the water surface is covered by ice sheet. Modulated or pulsed laser radiation, being absorbed in a layer of ice, results in the excitation of elastic perturbations, propagating on large distances over the surface and in the bulk of the ice canape and in the adjoining layer of liquid. Analytical expressions for the far-field acoustic field have been derived for the wavelengths of a laser source from decimal up to several microns. The diagram of directivity of acoustic radiation in liquid was computed for the frequency interval of modulation of laser intensity of $100 \mathrm{~Hz}$ to $15 \mathrm{kHz}$ [A. O. Maksimov, Tech. Phys. Lett. 22, 52-55 (1996)]. The particular attention was devoted to the description of radiation near the Mach angles of eigenmodes, propagating with veloci- 
ties exceeding the sound speed in liquid. It happened that the interference pattern near the Mach angle is very informative for the diagnostics of physical parameters of the ice plate [A. O. Maksimov, Acoust. Phys. 43, 577-582 (1997)].

3aUWc3. The ubiquitous FLEXBAR ${ }^{\mathrm{TM}}$ transducer element for lowfrequency, broadband high-power sonar projector arrays. James W. Fitzgerald (The Kildare Corp., One Spar Yard Rd., New London, CT 06320, kildare@connix.com)

The piezoceramic FLEXBAR ${ }^{\mathrm{TM}}$, a modified free-free flexure bar, is a sonar projector element of extraordinary versatility and superior performance. It represents a cost-effective, mature transducer technology that is uniquely suitable for projectors of virtually any configuration; viz., planar, cylindrical, spherical, or conformal. Piezoceramic FLEXBAR ${ }^{\mathrm{TM}}$ projectors have been designed to operate at frequencies from $\sim 250 \mathrm{~Hz}$ to $\sim 5 \mathrm{kHz}$, with source levels and beam patterns only constrained by the available power and the allowable array envelope. In conjunction with pressure-equalizing oil-filled internal cavities, FLEXBAR ${ }^{\mathrm{TM}}$ projectors are operational to essentially any depth. For example, REFLEXDUCER ${ }^{\mathrm{TM}}$ model RFD-420/A, a cylindrical array comprised of 32 FLEXBAR $^{\mathrm{TM}} \mathrm{s}$, and incorporated into a housing $20 \mathrm{~cm} \mathrm{D} \times 40 \mathrm{~cm} \mathrm{H}$, has the following performance characteristics: resonant frequency to $f_{0} \cong 2000 \mathrm{~Hz}$; quality factor $Q_{m} \cong 3$; bandwidth $\mathrm{B} / \mathrm{W} \cong 670 \mathrm{~Hz}(-3 \mathrm{~dB})$; source level SL $\cong 210 \mathrm{~dB} / / \mu \mathrm{Pa} / \mathrm{m}$; electric power in $\operatorname{Pwr}(e) \cong 12500 \mathrm{~W}$; acoustic power out $\operatorname{Pwr}(a) \cong 8125$; electro-acoustic efficiency $\eta_{\mathrm{ea}} \cong 65 \%$. The unit, including an internal 1:5 step-up transformer with a tuned secondary, weights approximately $66 \mathrm{~kg}$ so that its effective acoustic power-to-weight ratio is $\cong 120 \mathrm{~W} / \mathrm{kg}(\cong 55 \mathrm{~W} / \mathrm{lb}$ ). A conformal submarine-sail design is shown covering a bandwidth of $1-5 \mathrm{kHz}$ at a source level of $\mathrm{SL}$ $\cong 245 \mathrm{~dB} / / \mu \mathrm{Pa} / \mathrm{m}$, with steerable midband vertical beam patterns of $\theta_{v}$ $\cong 10$ degrees $(-3 \mathrm{~dB})$ and steerable horizontal patterns of $\theta_{h} \cong 5$ degrees $(-3 \mathrm{~dB})$.

3aUWc4. PZT5 spherical hydrophone simulation using a coupled FE-BE method. Soon S. Jarng (Dept. of Control \& Instrumentation, Chosun Univ., 375 Seoseok-Dong, Dong-Ku, Kwang-Ju, South Korea, ssjarng@ soback.kornet21.net)

This paper describes the application of a coupled finite-elementboundary element method to obtain the steady-state response of a hydrophone. The particular structure considered is a flooded piezoelectric spherical shell. The hydrophone is three-dimensionally simulated to transduce an incident plane acoustic pressure onto the outer surface of the sonar spherical shell to electrical potentials on inner and outer surfaces of the shell. The acoustic field formed from the scattered sound pressure is also simulated. In addition, the displacement of the shell caused by the exter- nally incident acoustic pressure is shown in temporal motion. The frequency response of the PZT5 spherical shell is also shown in comparison with theoretical expectation. The coupled FE-BE method is described in detail. [This study was granted by STEPI (Science \& Technology Policy Institute) Korea, as 1998 international program of co-work of research between Korea and the United Kingdom. The FORTRAN program of the present work was run by a super computer (Cray C90) operated at SERI (now ETRI), Korea.]

3aUWc5. A linear constrained design method for minimizing the interference effect in linear and planar array. Joon-il Song, KoengMo Sung (Appl. Acoust. Lab., Inst. of New Media and Commun., Seoul Natl. Univ., Kwanak-Ku, Seoul 151-742, Korea), and Jun-Seok Lim (Sejong Univ.)

In beamformer design, acoustic interaction effects experienced among the elements of an array will degrade its electroacoustic performance and change the beam patterns. An architectural modification has already been proposed as an effective measure for reducing this effect. Further reduction for interaction effects can be achieved by optimization techniques. This paper provides a new design method including the above constraints for linear or planar array. It is based on the solution of the linearly constrained least-squares problem and can be used in the arrays having arbitrary element locations. Results of computer simulations illustrating the performance of the beamformer are presented. [Work supported by UARC.]

3aUWc6. New sonar technologies using lower sound pressure levels. Kai Wicker and Egidius Arens (STN Atlas Elektronik GmbH, Sebaldsbrücker Heerstr. 235, D-28305 Bremen)

Depending on the conditions, modern active sonar equipment is capable of detecting targets up to distances of about $100 \mathrm{~km}$. To reach this goal, high sound-pressure levels up to $220 \mathrm{~dB}$ or more are usually necessary. Simultaneously, this sound-pressure level dramatically increases the probability of being detected, which represents a tactical disadvantage. A second problem arose in the last few years. Sometimes it is assumed that there could be some influences on the marine environment due to the high sound intensities. A new active sonar method and its signal processing is proposed. It could also be applied for acoustical oceanography applications, e.g., seafloor classification. By maintaining very wide frequency ranges during very long time periods, the high sound-pressure level can be reduced drastically. This results in a low probability of being intercepted and also in a reduced influence on the environment. For a realization of this method, four main topics have to be investigated: (1) improved broadband transducers, (2) special designed signal types, (3) a new signal processing concept, and (4) detailed calculations of sound propagation taking into account the local sea and seafloor properties. 


\title{
Medals and Awards Plenary Session
}

James E. West, Cochair

President, Acoustical Society of America
Joachim Scheuren, Cochair President, Deutsche Gesellschaft für Akustik

\section{Deutsche Gesellschaft für Akustik}

Lothar-Cremer-Preis

Helmholtz Medal

\author{
Acoustical Society of America \\ Presentation of Certificates to New Fellows of the Acoustical Society of America \\ Distinguished Service Citation to Elaine Moran \\ R. Bruce Lindsay Award to Paul E. Barbone \\ Helmholtz-Rayleigh Interdisciplinary Silver Medal to Jens P. Blauert \\ Honorary Fellowship to Leonid M. Brekhovskikh \\ Gold Medal to Henning E. von Gierke
}




\title{
Posters from various technical sessions remain on display in the Poster Gallery.
}

\section{Session 3pAAa}

\section{Architectural Acoustics and Signal Processing in Acoustics: Verification of Auralization and Modeling Programs I}

\author{
Michael Vorlaender, Cochair \\ Institut für Technische Akustik, Aachen University, D-52056 Aachen, Germany \\ Robert C. Coffeen, Cochair \\ Architectural Engineering, University of Kansas, Lawrence, Kansas 66045, USA
}

Chair's Introduction—4:15

Invited Papers

4:20

3pAAa1. Verification of prediction based on randomized tail-corrected cone-tracing and array modeling. Bengt-Inge L. Dalenbäck (CATT, Mariagatan 16A, SE-41471 Gothenburg, Sweden)

A new room acoustics prediction method, randomized tail-corrected cone-tracing (RTC), is described together with sample prediction/measurement comparisons. RTC is the result of ten years of experience using the image source model, ray- and conetracing, and combines elements of all three methods. RTC is a robust number-prediction method, including the effects of frequencydependent diffuse reflection for both early and late reflections, as well as a method that creates echograms suitable for binaural post-processing and auralization. Further, a WIN32 DLL directivity interface (DDI) is described. The interface is general and is capable of arbitrary data-format translation and accurate distance-dependent high-resolution array modeling including beam steering. The array modeling is either made via a generic DDI module or via modules custom designed based on detailed manufacturer array specifications (transducer placement, directivity pattern, aim, weighting filter, and delay). Sample prediction/measurements comparisons are given for DSP-controlled column arrays under free-field conditions, in a large railway station and in a large church. It is demonstrated that the common fixed measurement distance 10-deg full space data is insufficient for modeling arrays both due to the limited angular resolution and because far-field conditions may not be reached until $30 \mathrm{~m}$ distance or beyond.

4:40

3pAAa2. Report on the second International Round Robin on Room Acoustical Computer Simulation. Ingolf Bork (Projekt 1.401 Physikalisch-Technische Bundesanstalt, D-38116 Braunschweig, Germany)

A Swedish concert hall with a volume of about 11000 cubic meters served as a test object to compare the performance properties of room simulation software programs. In six octave bands and at 12 source-receiver combinations, nine acoustic parameters defined in ISO 3382 had to be calculated. In the first phase of this round robin only the geometry and a written description of the acoustically relevant properties were given, while in the second phase absorption and diffusion coefficients of the surfaces were prescribed for each octave band. The results obtained by the 16 participants from nine countries will be discussed and compared with measurements performed in the hall. The analysis of the discrepancies and the high variance of the calculated data show that the influence of the software user cannot be neglected and a high degree of practical experience in room acoustics is required to produce optimal results.

5:00

3pAAa3. Comparisons between computer simulations of room acoustical parameters and those measured in concert halls. Jens Holger Rindel (Norwegian Inst. of Bldg. Res., P.O. Box 123 Blindern, N-0314 Oslo, Norway), Hiroyoshi Shiokawa, Claus Lynge Christensen, and Anders Christian Gade (Tech. Univ. of Denmark, DK-2800 Lyngby, Denmark)

A number of European concert halls were surveyed in 1989. In this paper comparisons are made between measured room acoustical parameters and those obtained from computer simulations on concert halls using the ODEON program version 3.1. The key parameter compared with measured data is the reverberation time, and this is mainly used to adjust the absorption data of the surfaces in the computer model. But five additional parameters are calculated and compared with measured data as well. In order to determine the sensitivity of the computer model, comparisons are also made between the results obtained from computer simulations using models with a high geometrical fidelity and those from models with simplifications to geometry that are in better agreement with the laws of high-frequency modeling. 
3pAAa4. Real time auralization with EASE 3.0. Wolfgang Ahnert (ADA Acoust. Design Ahnert, Gustav-Meyer-Allee 25, D-13355 Berlin, Germany, wahnert@ada-acousticdesign.de)

The new EASE 3.0 for Windows combines advanced features of the DOS-based system EASE 2.1 with the known ones of the Auralization package EARS 1.1. In case of the DOS-based system, one has to use additional hardware to convolve the calculated impulse responses with the head-related transfer functions and eventually with the dry tests signal. After calculation of the corresponding impulse response one can listen now in EASE 3.0 to the convolved binaural signal by use of just a normal sound card. Additionally, with a duplex sound card one can enter CD signals directly and, after a transient time of $50 \mathrm{~ms}$ to $2 \mathrm{~s}$ (depending on the computer parameters and the sample rate of the signal), one can obtain the auralized results. Another option allows one to calculate the direct sound sequence of used speaker signals in realtime also and then to use the auralization routine to check echo occurrence or correct localization of sound sources. The convolution algorithm used in EASE 3.0 is patent pending and was created in cooperation with LAKE/Australia. In a project still under construction, the measured results have been compared with auralization results obtained by physical scale model measurements. Both results will be demonstrated.

$5: 40$

3pAAa5. Comparison of simulation and measured sound system performance in the German Reichstag Debate Hall, Berlin. Volker Loewer (IFB Rhein Main, Eleonorenstr. 11, D-65474 Bischofsheim, Germany, ifbrm@aol.com)

For the design of the new sound system for the Reichstag Debate Hall, Berlin, the ULYSSES computer aided acoustic design program has been used. The 3-D model of the room consisted of more than 1000 faces treated with different absorption in octave bands according to the architect's plans. Simulation of direct sound coverage, RT60, intelligibility, energy decay via ray-tracing, etc., was done to evaluate different solutions of the sound system designs and their interaction with room acoustics. It is intended to compare actual acoustic test results to simulated predictions and to discuss conformity and deviations. The software was also used to predict directivity balloons of the complex speaker arrays located in the debate hall. The balloons were derived from measured balloons of the single devices used in the arrays. Considering relative mechanical locations, acoustic centers, acoustic power, and delays, the predictions showed good conformity with polar measurements of a real cluster.

6:00

3pAAa6. A computer auralization and "real world" comparison using the computer programs EASE and EARS. Robert C. Coffeen (Architectural Eng., Marvin Hall, Univ. of Kansas, Lawrence, KS 66045) and Gabriel Caunt (Russ Berger Design Group, Inc., Dallas, TX 75244)

In order to compare a "real world" acoustic environment with a computer auralization of the same space, an Episcopal Church located in Prairie Village, Kansas (near Kansas City, Missouri) was selected as the "real" space. Anechoic speech and music recordings were reproduced in this space and recorded binaurally. The same anechoic recordings were introduced into a computer model of this room with auralizations produced by the computer programs EASE and EARS. The "real world" binaural recordings are compared with the computer-generated audio using both objective and subjective methods. The measured impulse response of the room and the computer-predicted impulse response are compared. Comparisons are also made between the measured and the predicted reverberation periods of the room.

WEDNESDAY AFTERNOON, 17 MARCH 1999

ROOM H1028, 4:35 TO 6:20 P.M.

\title{
Session 3pAAb
}

\section{Architectural Acoustics: Sound Insulation and Subjective Annoyance}

\author{
Jens Holger Rindel, Cochair \\ Norwegian Institute of Building Research, P.O. Box 123, Blindern, N-0314 Oslo, Norway \\ Angelo J. Campanella, Cochair \\ Campanella Associates, 3201 Ridgewood Drive, Columbus, Ohio 43026, USA \\ Chair's Introduction—4:35 \\ Invited Papers \\ 4:40
}

3pAAb1. On the utility of extending the low-frequency range of standards for sound isolation in buildings. Stephen J. Lind, Karl S. Pearsons, and Sanford A. Fidell (BBN Technol., 21128 Vanowen St., Canoga Park, CA 91303, slind@bbn.com)

Frequencies above $100 \mathrm{~Hz}$ are commonly considered in sound insulation computations as specified by ASTM E413 and ISO 717. This is appropriate for concerns about speech privacy in interior spaces. However, aircraft ground operations (including takeoff roll, engine run-ups, and thrust reverser deployment) may expose buildings near runways to appreciable amounts of energy at yet lower 
frequencies. Several studies of low-frequency aircraft noise levels have recently been completed in the United States, and the U.S. Federal Aviation Administration is paying for architectural treatments to reduce low-frequency aircraft noise in residences near one airport. This paper describes the findings of a recent social survey of residential annoyance caused by low-frequency runway sideline noise, including noise in the 25 to $80 \mathrm{~Hz}$ one-third octave bands, and reviews other evidence about the role of low-frequency energy in sound isolation metrics.

5:00

3pAAb2. On subjective impact sound insulation classes. Per Hammer (Dept. of Eng. Acoust., Lund Univ., Box 118, 22100 Lund, Sweden, per.hammer@kstr.lth.se) and Erling Nilsson (Lund Univ., 22100 Lund, Sweden)

The possibility to divide impact sound annoyance into classes is investigated. The experiments, based on human perception, are done in a laboratory environment where various floor structures have been included to produce impact noise. These tests are correlated to studies pertained to real-life situations for tenants. The statistical tool to test the significant differences between the classes is Rou-Kupper. Real footsteps are used as a noise source. In addition, airborne sound insulation is treated in a similar manner, whereby one may conclude that there are some difficulties finding classes with statistical significant differences. [Work supported by the Swedish Council of Building Research.]

\section{Contributed Papers}

\section{$5: 20$}

3pAAb3. Group subjective ratings of airborne sound insulation. John Bradley (IRC, Natl. Res. Council, Montreal Rd., Ottawa, ON K1A 0R6, Canada, john.bradley@nrc.ca)

This paper will compare various sound insulation measures as predictors of the grouped subjective ratings obtained from a field survey of 600 subjects and the airborne sound insulation of their 300 party walls. The subjects lived in both row housing and multi-floor apartment buildings in three different Canadian cities. The questionnaire first asked about general issues concerning the subjects building followed by more specific questions rating the sound insulation and the audibility of various noises. Sound transmission loss measurements were made in $1 / 3$ octave bands from 100 to $4000 \mathrm{~Hz}$. In addition to the standard ISO and ASTM single number ratings, 20 different single number sound isolation measures were calculated. Most were simple variations of the standard quantities. The standard STC rating (including the 8-dB rule) was one of the better predictors of most responses. Excluding the 8-dB rule decreased correlations with responses and especially those concerning hearing musical sounds. Several responses were best correlated with transmission loss values in the $160-$ and $200-\mathrm{Hz}$ bands.

\section{5:40}

3pAAb4. Considerations about the influence of traffic noise in the design of new hospitals. Eduardo Bauzer Medeiros (Dept. de Engenharia Mecânica da UFMG, Av. Antônio Carlos 6627, 31270-901, Belo Horizonte-MG, Brazil, flugzbau@dedalus.lcc.ufmg.br) and Ivan de Azevedo Camelier (UBI, 6200, Covilhã, Portugal)

The main origin of noise disturbance in new hospitals located inside densely populated areas can usually be associated with vehicular traffic. However, the effect of this disturbance is often not fully appreciated. Compliance with standard legislation limits is often considered to be a satisfactory design criterion. In fact, even moderately higher noise levels can introduce physiological changes, which may have a decisive influence on the convalescence of a hospital patient. The present work associates some of the information which is available on the effects of patients subjected to different noise levels with considerations about hospital and the associated access roads design. Field measurements carried out in the vicinity of typical existing and planned hospitals are also described and associated with the local traffic conditions. Finally, a few practical guidelines are laid, considering a rest situation of a hospital under construction near an important access road. The objective is to establish a set of acoustic design guidelines for hospitals and the surrounding areas, particularly the road system, aiming at a more efficient convalescence period for the patients.

\section{6:00}

3pAAb5. Vibrations caused by construction work-Measurements and surveillance. Wolfgang Teuber and Ernst-Jo Voelker (Inst. for Acoust. and Bldg. Phys., Oberursel, Germany)

Numerous construction principles applied especially in underground engineering are the sources of vibrations. The measurement of vibrations and evaluation of the results are made based upon different criteria. These criteria are formed taking into consideration the influences upon people in the surrounding area, the risk of damage to buildings and the possibility of the malfunctioning of technical systems such as computers or laser equipment. Measurements were taken while several different construction methods were being applied. Short-term measurements are, for the most part, insufficient and must be completed by long-term research. Maximum acceptable values are defined in existing standards based on the influences of vibrations upon persons and upon various kinds of buildings. For technical equipment, stricter limits had to be defined. When the type and location of construction work changes, the application of an automatic monitoring system is advantageous. Such a system is described which was installed to survey computers with special hard disc drives. Existing measurement results allow for predictions and calculations to be made in order to determine the best method of construction. Some principles of building methods, measurements, and vibration monitoring are mentioned. 


\title{
Session 3pAO
}

\section{Acoustical Oceanography and Underwater Acoustics: Acoustic Tomography and Thermometry II}

\author{
James F. Lynch, Chair \\ Woods Hole Oceanographic Institute, Bigelow 203, Woods Hole, Massachusetts 02543, USA
}

\section{Invited Papers}

$$
\text { 4:20 }
$$

3pA01. Shallow-water tomography: Acoustic maps of the New England shelfbreak front. Ching-Sang Chiu (Dept. of Oceanogr., Naval Postgrad. School, Monterey, CA 93943), James F. Lynch, and Glen Gawarkiewicz (Woods Hole Oceanogr. Inst., Woods Hole, MA 02543)

In the summer of 1996, an integrated acoustic-oceanographic experiment was carried out in the Middle Atlantic Bight to study the dynamics of the shelfbreak front and the effects of the frontal variability on sound propagation. The experiment employed a suite of acoustic and oceanographic sensors, including several sources and two vertical hydrophone arrays (VLAs) straddling the shelfbreak front and a SeaSoar that provided several high-resolution, three-dimensional surveys of the frontal region. In this presentation, the results of an acoustic tomographic analysis of the frontal variability are reported. In deriving the tomographic maps, an adaptive beamformer was first used to detect, resolve, and track individual acoustic ray and modal arrivals from a 224- and a 400-Hz source on the slope to a vertical array on the shelf. A hybrid ray-mode inverse technique was then applied to the resolved arrivals to produce a time series of cross-frontal images of ocean temperature. The tomographic observations are interpreted together with the SeaSoar, ADCP, and thermistor data. [Work supported by ONR.]

\section{4:40}

3pA02. Broadband geo-acoustic inversion in shallow water. Jean-Pierre Hermand (Environ. Res. Div. SACLANT Undersea Res. Ctr., viale San Bartolomeo 400, 19138 La Spezia, Italy, hermand@saclantc.nato.int)

The paper discusses an inversion method that allows the rapid determination of in situ geo-acoustic properties of the ocean bottom without resorting to large acoustic receiving apertures, synthetic or real. The method is based on broadband, waterborne measurements and modeling of the waveguide impulse response between a controlled source and a single hydrophone. Results from 1994-1997 experiments in Mediterranean shallow waters with single elements of vertical array and advanced drifting acoustic buoys are reviewed. Inversion of the bottom parameters is performed with an objective function that includes the processing gain of a model-based matched filter (MBMF) receiver relative to the conventional matched filter. The MBMF reference signals incorporate waveguide Green's functions for the known geometry and water column acoustic model, and hypothesized bottom geo-acoustic models. The inversion results demonstrated that—even for the fairly complex environmental conditions of the experiments—a single transmission of a broadband coded signal received at a single depth and a few hundred of forward modeling runs were sufficient to resolve the bottom features. These included the sound-speed profile, attenuation, density, and thickness of the top sediment layer, and sound speed and attenuation of the bottom. Exhaustive parameter search proved unequivocally the low-ambiguity and high-resolution properties of the MBMF-derived objective. The single-hydrophone bottom inverses closely agree with the ones obtained under the exact same conditions from matched-field processing of multitone pressure fields sampled on a vertical array. Both of these results agree with expectations from geophysical ground thrust.

\section{Contributed Papers}

\section{5:00}

3pAO3. Results for tomographic geoacoustic inversion on simulated multiarray data. A. Tolstoy (Integrated Performance Decisions, Inc., 8610 Battailles Ct., Annandale, VA 22003, atolstoy@ipdinc.com)

Only recently have broadband multiarray data been collected which are suitable for tomographic inversion for the estimation of shallow-water geoacoustic parameters. In particular, this unique data set was collected in the Haro Strait region off the west coast of Canada by N. R. Chapman and colleagues. These data have now been simulated and studied in preparation for the tomographic inversion of the actual data, a very difficult problem involving hundreds of parameters. Results to be discussed here con- cern these simulated data and recent successes in their inversion for the estimation of such properties as sediment sound-speeds and densities over a region where such parameters vary as a function of range, azimuth, and depth. [Work supported by ONR.] $\mathbf{6}: 20$

3pAO4. Acoustic data preprocessing in the TOMOLAB/OCTOPUS project. Mauuary Didier and Bernard Faure (Laboratoire des images et signaux, INP, Grenoble, St. Martin d'heres, France, Didier.Mauuary@lis.inpg.fr)

This paper presents the acoustic data preprocessing task in the TOMOLAB graphic interface tool. TOMOLAB is a goal of the OCTOPUS project, under the MAST III program of the European Com- 
munity. Data preprocessing consists in transforming raw tomographic data into compressed/correlated peaklike data. It is the first step before time delay estimation, time delay identification, and time delay inversion. The complete data processing must perform matched filtering, multisequence averaging, Doppler/phase compensation, and mooring motion correction. A synthetic view is given of several technical choices that integrate these tasks, jointly or successively. This work is based on internal studies and existing pieces of literature. The Hadamard transform, the Fourier matched filtering, fast Doppler processing, phase estimation/correction algorithm, delay shifting in the time or Fourier domain, data format and sampling are discussed. Efficient data processing has to account for the computing speed, the compression ratio, and achievable accuracy of delay estimation.

$5: 40$

3pAO5. Matched-peak tomography. E. K. Skarsoulis (Inst. of Appl. and Computational Mathematics, FORTH, 71110 Heraklion, Crete, Greece)

A new approach is presented for the inversion of travel-time data in ocean acoustic tomography. Using model relations between sound-speed and arrival-time perturbations about a set of background states, arrival times and associated model errors are calculated on a fine grid of model states discretizing the parameter space. Each model state can explain (identify) a number of observed peaks in a particular reception laying within the uncertainty intervals of the corresponding predicted arrival times. The model states that explain the maximum number of observed peaks are considered as the more likely parametric descriptions of the reception; these model states can be described in terms of mean values and variances providing a statistical answer (matched-peak solution) to the inversion problem. An advantage of the proposed approach is that there is no need for initialization of the inversion procedure, i.e., no need for additional hydrographic information. Furthermore, the matched-peak solution for each reception is independent of the previous reception identification/inversion results. The method was applied to nine-monthlong tomography data from the Thetis- 2 experiment in the western Mediterranean sea. [Work partially supported by EU/MAST.]

\section{6:00}

3pAO6. On the simultaneous estimation of current and sound velocity structure in the ocean, using matched-field processing. Michael Taroudakis (Dept. of Mathematics, Univ. of Crete and Inst. of Appl. and Computational Mathematics, FORTH, P.O. Box 1527, 71110 Heraklion, Crete, Greece, taroud@iacm.forth.gr) and Maria Markaki (Inst. of Appl. and Computational Mathematics, FORTH, 71110 Heraklion, Crete, Greece)

A matched-field processing method for the simultaneous estimation of ocean current velocity structure and sound speed profiles, based on reciprocal transmissions of $\mathrm{cw}$ or broadband acoustic signals between two locations in the water column, is presented and discussed. For each transmission, a single source and a vertical array of hydrophones are used. The current velocity is introduced as an additional unknown in the inverse problem, through the concept of the "effective sound speed," being the sum of the actual sound speed and the projection of the current velocity on a vertical plane, defined by the sound source and the receiver. The work is mainly focused on the study of the effect of variations of the current velocity on the various processors proposed for the matched-field inversions and on the inversion procedure itself, under the assumption that neither the actual position of the source receiver system nor the actual sound velocity structure are known. Using synthetic data, it is demonstrated that the results of inversion for the current velocity are reliable, under some limitations posed by the degree of uncertainty for the other unknown parameters. [Work supported by INTAS-RFBR.]

\section{Contributed Posters}

These posters will be on display in the Poster Gallery from Thursday to Friday, 18-19 March. Authors will be at their posters from 10:00 a.m. to 12:00 noon on Friday, 19 March.

3pAO7. The experimental demonstration of selective mode excitation in a shallow-water environment. James C. Preisig (Dept. of Appl. Ocean Phys. and Eng., Woods Hole Oceanogr. Inst., Woods Hole, MA 02543), John R. Buck (Univ. of Massachusetts, North Dartmouth, MA 02747), Mark Johnson (Woods Hole Oceanogr. Inst., Woods Hole, MA 02543), and Hu Dou (Univ. of Massachusetts, North Dartmouth, MA 02747)

The ability to selectively excite single acoustic modes in a shallowwater environment is demonstrated and analyzed. Using vertical source and receiving arrays and the feedback control techniques based on those in Buck et al. [IEEE J. Oceanic Eng. 22, 281-291 (1997)], acoustic modes $1-3$ were excited sequentially for periods up to $20 \mathrm{~min}$ with a fidelity of approximately $60 \mathrm{~dB}$ for mode 1 to $25 \mathrm{~dB}$ for mode 3 . The filtering of the received signal used in the feedback loop by projection into the subspace spanned by the dominant system modes was found to significantly improve the system's performance. This result will be explained and analyzed. The requirement for phase stabilization of the sampled received signal is also presented. The ability demonstrated here for high fidelity selectively controlled modal content of a transmitted acoustic signal offers potential for new methods for acoustic tomography and for studying underwater acoustic propagation. [Work supported by ONR Ocean Acoustics.]
3pAO8. Model of acoustic monitoring of heat and mass transport in the Fram Strait. Konstantin A. Naugolnykh (Environ. Technol. Lab., Boulder, CO 80303), Ola M. Johannessen (Nansen Environ. and Remote Sensing Ctr., Bergen, Norway), Igor B. Esipov, Oleg B. Ovchinnikov, and Yuri I. Tuzhilkin (N. Andreyev Acoust. Inst., Moscow, 117036 Russia)

A computation model of acoustical monitoring of heat and mass transport in the Fram Strait is discussed. The method relies on the advections of small-scale inhomogeneities across the acoustic path and travel-time variations in the process of signal crossing of the Strait on a number of paths to infer the intervening of fine-scale variability and transverse current. Inhomogeneities of the current produce perturbations in the travel time of the sound, and the current can be sensed by generating a time-lagged cross correlation of the full acoustic field. Both the linear four-element transmission array and four-element receiving array with equally spaced elements were used for calculations. By combining the signals from each transmitter-receiver pair in different ways, a number of different path positions were probed and a profile of transverse current $U(r)$ along the propagation path $r$ was retrieved, whereas the value of the travel-time variation is related with $T$-temperature change. The possibility of estimation of heat $Q \sim \int U(r) T(r) d r$ and mass $M \sim \int U(r) d r$ transport in the cross section is regarded. [Work supported by Environmental and Climate Programme of EC, Project PL970338.] 
3pA09. Internal wave inversions for one year of Pioneer-Hawaii ATOC transmissions. Kevin D. Heaney and the ATOC Group ${ }^{\text {a) }}$ (Sci. Applications Intl. Corp., 11803 Sorrento Valley Rd., San Diego, CA 92121)

The ATOC experiment transmitted $75-\mathrm{Hz}$ signals from Pioneer Seamount off the coast of California to a vertical line array (VLA) off Hawaii. The combination of VLA and long-range propagation provides the opportunity to perform mode-based internal wave tomography. Each arrival is mode-filtered and the statistics of the mode arrivals are tabulated. These individual mode arrival time (centroid) and spread are then compared with calculated mode statistics from broadband PE modeling of the propagation path. In this manner a statistically robust inversion for the internal wave strength is performed. The weakness of the inversion is the sensitivity to the bottom scattering near the source. Results for one year of data will be presented for two different bottom types. The long-scale time series may indicate possible sources of internal waves in the ocean. Small time scale variations may be attributable to internal tides. ${ }^{\text {a) }}$ The ATOC Group: A. B. Baggeroer, D. Menememlis, and C. Wunsch (MIT); T. Birdsall, K. Metzger (Univ. of Michigan); C. Clark (Cornell Univ.); J. Colosi (WHOI); B. D. Corneulle, M. Dzieciuch, W. Munk, P. F. Worcester (SIO); D. Costa (Univ. of California, Santa Cruz, CA); B. D. Dushaw, B. M. Howe, J. A. Mercer, and R. C. Spindel (APL-U Wash.); A. M. G. Forbes (CSIRO, Hobart).

3pA010. AMOC: Preliminary results. Hanne Sagen (NERSC, Edv. Griegsvei 3A, 5037 Solheimsviken, Norway, hanne.sagen@nrsc.no), Ola M. Johannessen, Halvor Hobaek, and Sigve Tjoetta (Univ. of Bergen, Bergen, Norway)

The scope of this presentation is to give preliminary results of the sensitivity study of sound propagation in the Arctic including the Fram Strait using propagation loss observations and existing acoustic propagation models. Special attention to the consequences for design of future acoustic monitoring concepts will be made. One of the important consequences of the preliminary results is the conflict between the optimum transmission frequency and the optimum frequency for retrieving information about the ice cover. If an experiment is designed to propagate over long distances at a relatively low frequency, then the reflection from a "smooth" ice cover is total and there will be no information about the ice thickness or internal properties of the ice cover. The attenuation due to the ice will be dominated by scattering from rough surface (large facets). So this type of experiment will give information about the averaged temperature through the travel times, while the ice information will be limited. Therefore two concepts have to be considered: one to measure ice parameters, which will need signals composed by appropriate frequencies, and another concept to measure averaged temperature, which needs low frequencies insensitive to ice cover.
3pAO11. Method of the main informative components for acoustic tomography. Alexander Ya. Kalyuzhnyi (Inst. of Hydromechanics, 8/4 Zhelyabov Str., Kiev, 252057, Ukraine) and Leonid G. Krasny (Ericsson, Inc., RTP, NC 27709)

Techniques for an ocean acoustic tomography are based on the representation of the sound-speed field (SSF) by an expansion of some orthogonal basis functions. It is usually assumed that the most efficient basis functions for the SSF expansion are so-called empirical orthogonal functions (EOF). Meanwhile, the experiments show that algorithms based on the EOF can be extremely sensitive to slight errors in the assumed signal and noise characteristics. A new improved algorithm for acoustic tomography will be presented which minimizes output statistical errors of the SSF estimation. The concept of this algorithm is to use eigenvectors of the Fisher information operator as the basis functions into the SSF expansion. The algorithm involves a quadratic inequality constrain on the SSF expansion coefficients to select the most informative components among all the eigenvectors. It is shown that it leads to a robust algorithm in which statistical errors do not accumulate. The performance of the proposed algorithm will be illustrated both for deterministic and random signals.

3pAO12. An ocean acoustic tomography experiment in the central Labrador Sea-First results. Detlef Kindler and Uwe Send (Inst. für Meereskunde, Univ. Kiel, Düsternbrooker Weg 20, D-24105 Kiel, Germany)

First results of an ongoing acoustic tomography experiment will be presented. Since August 1996 a mooring array consisting of four tomography transceivers $(400 \mathrm{~Hz})$, redeployed in the summers of 1997 and 1998, has been measuring acoustic travel times over distances of typically 170$300 \mathrm{~km}$ in the central Labrador Sea. The purpose of such measurements is to estimate time series of horizontally integrated properties like ocean heat content and vertical temperature stratification by inverting travel times into sound speeds and hence into heat contents and temperatures. These large-scale properties will be used to study the interannual variability of ocean heat budget and water masses formed in close connection to deep convection and the ensuing renewal of Labrador Sea Water (LSW) [R. A. Clarke and J. C. Gascard, J. Phys. Oceanogr. 13, 1764-1778 (1983)]. The variability during winter results from destabilization of the water column near the ocean surface caused by atmospheric cooling, subsequent mixing down to $1000-2000 \mathrm{~m}$, and the following restratification of the convection area. The investigations are focused on the upper $2000 \mathrm{~m}$ of the water column, which are well insonified by several trackable ray paths with enough acoustic energy to be processed. [Work supported by Deutsche Forschungsgemeinschaft.] 


\title{
Session 3pEA
}

\section{Engineering Acoustics and Underwater Acoustics: Recent Advances in Underwater Transducers II}

\author{
Elizabeth A. McLaughlin, Cochair \\ Naval Undersea Warfare Center, Code 2131, Newport, Rhode Island 02841-1708, USA \\ Didier Boucher, Cochair \\ DCN Ingénierie Centre SUD, Sous-Direction Lutte Sous La Mer, BP 30, 83800 Toulon Naval, France
}

\section{Contributed Papers}

4:00

\begin{abstract}
3pEA1. Application of the Tonpilz design for optimizing parameters of rod transducers. Boris Aronov (Acoust. Res. Lab., Dept. of Elec. and Computer Eng. and Ctr. for Marine Sci. and Tech., Univ. of Massachusetts, Dartmouth, North Dartmouth, MA 02747)
\end{abstract}

The optimum design of a rod transducer under various loading conditions and operating requirements is examined. It is often possible that the operational requirements can be met by using only a part of the rod volume with the electromechanical or piezoelectric element. The remaining sections of the rod can be replaced by passive materials or caps (i.e., a "head" and a "tail") while still maintaining the same input impedances and resonant frequency. Such a composite rod transducer made of active and passive materials having different length and cross-sectional areas is of the "Tonpilz" type. The proper choice of dimensions and materials for the caps will result in optimum transformation of mechanical (acoustical) loads and transducer performance. Both criteria of optimization, the rod transducer parameters and the procedure for determining properties of the caps that provides optimal loads, are discussed.

4:20

3pEA2. Large aperture 1-3 composite longitudinal resonator (tonpilz) transducer. Charles W. Allen, W. Jack Hughes, and David J. Van Tol (Appl. Res. Lab., Penn State Univ., P.O. Box 30, State College, PA 16804)

A large aperture underwater transducer utilizing 1-3 piezoelectric composite material that operates as a longitudinally resonant (tonpilz) device with a resonance frequency of $45 \mathrm{kHz}$ has been fabricated and tested. The transducer consists of continuous plates of steel and aluminum that act as the rear and front masses, respectively, and a motor section of continuous 1-3 PZT-5H composite. The active radiating face (aluminum front mass) has dimensions of 6.3 in. (4.7 wavelengths) and $3.9 \mathrm{in.} \mathrm{(2.9} \mathrm{wavelengths).}$ Conventional transducers or arrays with radiating faces that have dimensions of over one wavelength require dicing or separate elements to reduce the effect of lateral modes in the components. The tonpilz transducer utilizing 1-3 composite material requires no dicing of any of its components, so fabrication of large aperture arrays that only require a single forward-looking narrow beam are greatly simplified. The measured acoustic responses versus frequency and angle demonstrate that there are no extraneous modes that affect the acoustic performance of the transducer and they compare well to theory for a one-dimensional longitudinal resonator. [Work sponsored by the Scripps Institution of Oceanography, University of California, San Diego.]
4:40

3pEA3. The development of a doubly curved acoustic array using injection molded 1-3 piezocomposite. Kim C. Benjamin, Stephen F. Oliver, Jose C. Arteiro (Naval Undersea Warfare Ctr. Div. Newport, 1176 Howell St., Newport, RI 02841, benjaminkc@code20nl.npt.nuwc.navy.mil), Russell S. Petrucci, and William J. Serwatka (Mater. Systems, Inc., Littleton, MA 01460)

The design, fabrication, and acoustic calibration of a doubly curved acoustic array for UUV oceanographic applications is presented. The sensor employs a unique piezoceramic thermoplastic composite which is conformable and capable of deep ocean operation. The composite consists of several small diameter $(1.0 \mathrm{~mm})$ PZT-5H rods regularly distributed in a two-dimensional lattice and aligned perpendicular to their diameter. The PZT-5H rods are encapsulated within a thermoplastic viscoelastic moldable epoxy matrix to form an active acoustic composite panel. The thermoplastic epoxy phase of the composite allows the panel to be shaped to a specific vehicle geometry. Low-profile $(<1.0 \mathrm{~mm})$ coaxial cable embedded within the composite panel provides addressing between the acoustic array elements and the processing electronics. [This work was supported by Code 80 of the Naval Undersea Warfare Center, Division Newport.]

5:00

3pEA4. Ultra-wide bandwidth backscattering measurements using a transparent flat PVDF sheet source. Scot F. Morse (Code 7136, Naval Res. Lab., Washington, DC 20375), Karen Gipson (Univ. of Puget Sound, Tacoma, WA 98416), Greg Kaduchak (Los Alamos Natl. Lab., Los Alamos, NM 87545), Kevin L. Williams (Univ. of Washington, Seattle, WA 98105), Brian T. Hefner, and Philip L. Marston (Washington State Univ., Pullman, WA 99164-2814)

A thin extended flat PVDF film surrounded by water is demonstrated to be suitable for exploring scattering responses of complicated targets over a range of frequencies from below $2 \mathrm{kHz}$ to $1000 \mathrm{kHz}$. The target lies close to the source, and in the configuration normally used, the scattering passes through the source to a distant hydrophone. While initial experiments [Kaduchak et al., J. Acoust. Soc. Am. 97, 2644-2708 (1995)] explored the resonances of spherical shells for which analytical solutions are known, recent experiments have explored novel features in the responses of truncated tilted cylinders that are either hollow [Morse et al., J. Acoust. Soc. Am. 103, 785-794 (1998)] or solid [K. Gipson, Ph.D. thesis, Washington State Univ. (1998)]. The spectrum radiated by the sheet can be altered by using voltage-step, voltage-pulse, or weighted chirp excitations. An equivalent circuit model is useful for describing the radiated spectrum. Crisp tone bursts have also been produced and used to study the lowfrequency resonances of a steel shell in Lake Union (Seattle). [Work supported in part by the Office of Naval Research and by APL.] 
compression strength. Evidence of damage was found at stresses as low as one-third of the compressive strength. [Work supported by ONR and SPAWAR.]

3pEA5. Mechanical properties of lead magnesium niobate/lead titanate ceramics. Elizabeth A. McLaughlin and Lynn Ewart (Naval Undersea Warfare Ctr., Code 2131, 1176 Howell St., Newport, RI 02841)

This research examines the mechanical properties of doped electrostrictive lead magnesium niobate / lead titanate (PMN-PT). While optimization of the electromechanical properties has been the focus of the materials development effort, mounting anecdotal evidence indicates that there are problems with the mechanical integrity of PMN-PT. Mechanical testing has been employed (1) to measure the flexural strength of doped PMN-PT and understand the causes of failure, and (2) to measure the compression strength of PMN-PT and identify the onset of microstructural damage. Navy type-III ceramic (PZT8) was measured as a benchmark. The average flexural strength of poled PZT8 was found to be $110 \mathrm{MPa}$. Average flexural strengths ranging from 44 to $60 \mathrm{MPa}$ were measured for batches of barium- and lanthanum-doped PMN-PT. Fractography identified the causes of failure. Process modifications, by one vendor, have increased the flexural strength by $38 \%$. The average compression strength of unpoled PZT8 was found to be $1040 \mathrm{MPa}$. An average compressive strength of $890 \mathrm{MPa}$ was measured for barium-doped PMN-PT. Prior to failure, a ceramic develops microstructural damage at levels below the

\section{$5: 40$}

3pEA6. Evidence of nonlinear losses in electrostrictive materials. Harold C. Robinson (Naval Undersea Warfare Ctr., Newport, RI 02841)

It has been well established that the equivalent piezoelectric properties used to model the large signal properties of electrostrictive materials such as lead magnesium niobate/lead titanate (PMN/PT) are functions of the operating conditions. Since these materials are nonlinear, DC bias field, AC drive field, prestress level, and temperature all affect the piezoelectric constants and the coupling factor. However, in transducer models based on equivalent circuits, the dielectric loss is represented as a constant under all of these conditions. Moreover, the dielectric loss tangent typically used in these models must either be inferred from transducer data or be measured under conditions (such as using an RLC meter) that do not represent the actual conditions of operation. This paper will present data, from both material and transducer measurements, suggesting that the losses in electrostrictive materials are strong functions of the temperature, drive field, and compressive prestress. Possible approaches to obtaining improved loss measures from material measurements will be discussed.

\title{
Session 3pED
}

\section{Education in Acoustics: Take Fives-Sharing Ideas for Teaching Acoustics}

\author{
Uwe J. Hansen, Cochair \\ Department of Physics, Indiana University, Terre Haute, Indiana 47809, USA \\ Armin Kohlrausch, Cochair \\ IPO Center for Research on User-System Interaction, P.O. Box 513, NL-5600 MB Eindhoven, The Netherlands
}

Do you have a novel demonstration, a new laboratory experiment, a favorite video, a recorded sound example, or a new idea for teaching acoustics which you are willing to share with your colleagues? At this session a sign-up board will be provided for scheduling presentations. No abstracts are printed. Presenters are encouraged to have handouts to distribute. Multiple presentations are acceptable (not consecutively). Presentations are limited to 5 minutes. Keep them short! Keep them fun! 
Session 3pMU

\title{
Musical Acoustics: Musical Instruments and Structural Acoustics II: Piano and Related Instruments
}

\author{
Isao Nakamura, Cochair \\ 1-33-25 Kokuryo, Chofu, Tokyo 182-0022, Japan \\ Ingolf Bork, Cochair \\ Physikalische Technische Bundesanstalt, Bundesallee 100, D-38116 Braunschweig, Germany \\ Chair's Introduction-4:15 \\ Invited Papers \\ 4:20
}

3pMU1. Piano string excitation: The question of gravity influence on the sound spectra. Anatoli Stulov (Dept. of Mech. and Appl. Mathematics, Inst. of Cybernetics, Akadeemia tee 21, Tallinn, EE0026, Estonia)

The influence of gravity on the process of the grand piano hammer-string interaction was appreciated by D. E. Hall [J. Acoust. Soc. Am. 79, 141-147 (1986)]. Using the reasonable physical assumptions, it was shown that the gravity influence is small and may not be taken into account. It is obvious, but it is important to know exactly what this influence is. The vibration spectra of different flexible strings excited by piano hammers in the gravity field and without gravity were analyzed here. Of course, the influence of gravity increases with the increasing of the contact time. Its depends on the hammer velocity; therefore, the string excitation by hammers with velocities less than $0.5 \mathrm{~m} / \mathrm{s}$ was considered. It was shown that: the gravity influence is greater for the smooth hammers, and this influence is more essential for the middle range of notes; for the small velocities of hammers the gravity changes the mode energy levels of the primary harmonics up to $10 \mathrm{~dB}$, if the hammer strikes only one string of the triple set; the gravity must be taken into account definitely, if the contact time is greater than $5 \mathrm{~ms}$.

4:40

3pMU2. Experimental analysis of two-dimensional vibration of a piano string measured with an optical device. Hideyuki Tanaka, Keinosuke Nagai, and Koichi Mizutani (Inst. of Appl. Phys., Univ. of Tsukuba, Tsukuba Ibaraki, 305-8573 Japan)

It is well known that a piano string vibrates in the direction not only perpendicular to the soundboard but also parallel to it. The sound decays rapidly at first and then it decays slowly. A change in the predominant direction of vibration of the string from perpendicular to parallel contributes to the compound decay rate [G. Weinreich, Sci. Am. 240, 118-127 (1979)]. In this paper, two-dimensional vibration of a piano string is measured with handmade equipment consisting of two phototransistors. Just after the string was struck with a hammer, it vibrates perpendicularly to the soundboard. Then it vibrates gradually in the direction parallel to the soundboard. As a result, a point of the string moves on an elliptic trajectory. It was found that the fundamental resonant frequency of the perpendicular vibration of the E1 string, for example, which is about $40 \mathrm{~Hz}$, is $0.07 \mathrm{~Hz}$ lower than that of parallel vibration. This causes the change of rotating direction of the string every $7 \mathrm{~s}$. The discrepancy of the resonant frequencies is also found in the vibration of the $\mathrm{D} \# 1$ string.

\section{5:00}

3pMU3. Damping of string motion, a significant parameter for sound quality. Klaus Wogram (Phys.-Techn. Bundesanstalt, Proj. 1.401, Bundesallee 100, D-38116 Braunschweig, Germany, klaus.wogram@ptb.de)

To improve the sound quality of pianos and upright pianos the piano maker mostly plans a change of the construction of the backs and the soundboard with its ribs, bridges, and back posts. Unfortunately he often disregards that all these efforts cannot be successful if the damping of the strings is insufficient. Its influence on the sound quality can be very much stronger than that of the soundboard. Guided by some examples, the importance of the dampers, their construction, their mass, and their position will be discussed. In addition the reduction of sound quality due to an insufficient damping of the not-speaking part of a string will be demonstrated.

\section{$5: 20$}

3pMU4. The influence of inharmonicity on piano tuning. Taro Mori and Ingolf Bork (Physikalisch-Technische Bundesanstalt, Bundesallee 100, 38116 Braunschweig, Germany, taro.mori@ptb.de)

It is well known that octaves of piano sounds are stretched to more than a $2: 1$ ratio. One reason is that partials of piano tones are not exactly harmonic because of the flexural rigidity of the string. This inharmonicity is characterized by the coefficient $B$, which depends on the tension, length, Young's modulus, the area of cross section, and the radius of gyration of the string. Piano technicians are trained to tune the octave as "beautifully" as possible, but the inharmonicity of the string hinders perfect tuning without beat. For example, when the second partial of the lower sound and the first partial of the upper sound are tuned without beat, all other partial pairs show beats. On the other hand, since the timbre structure of partials is unique for each instrument, the tuned octaves of two different instruments may differ. Some piano sounds were simulated by additive synthesis with different octave, inharmonicity, and timbre in order to perform subjective hearing tests with some piano tuners. It could be shown that the tuned octave width depends not only on the coefficient $B$ but also on the timbre. The influence of the sound board is also discussed. 
3pMU5. Psychoacoustic model for calculating the sound quality of piano tones. Miriam N. Valenzuela (Inst. of Mech., Faculty of Aerosp. Technol., Univ. of the Federal Armed Forces, Munich, D-85577 Neubiberg, Germany, miriam.valenzuela@unibw-muenchen.de)

Assuming that only audible dissimilarities between different piano tones may lead to different quality judgments, experiments were conducted to determine the perceptual attributes listeners use to judge these dissimilarities. The psychoacoustic results showed that two major attributes contribute with over $90 \%$ to the explanation of the perceived dissimilarities: the psychoacoustic "sharpness" and a second attribute that was described with the scale "open-closed." Both attributes depend on the specific loudness distribution of the sound signals and are defined as a weighted first moment of the critical-band rate distribution of specific loudness using different weighting functions. The weighting function used to calculate the second attribute emphasizes spectral components around $1 \mathrm{kHz}$. The model developed for calculating audible dissimilarities between piano tones showed a good agreement between psychoacoustically measured and calculated dissimilarities; the correlation was 0.90 . The influence of the two attributes on sound quality judgments was verified by listening tests with appropriately modified piano sounds. The results showed that calculating the sound quality of musical instruments on the basis of their sound signals requires an aurally adequate signal processing. The model developed to calculate the sound quality of piano tones showed a good agreement between measured and calculated quality orders.

\title{
Contributed Paper
}

\section{6:00}

3pMU6. Stiffness of strings: Significance in musical acoustics. Edgar Lieber $^{\text {a) }}$ (former member of "Institut für Musikinstrumentenbau," D-08267 Zwota, Germany)

In a linear differential equation of a vibrating string there is a link representing stiffness of the string. In nonlinear theory, string stiffness has not yet been taken into consideration. The author has completed this by means of the Lagrange II method and presents essential results. The significance of string stiffness is different for the different groups of instru- ments. Pianos have remarkably stiff strings. This is inevitable and also necessary because stiffness participates essentially in forming vividness of sound. The well-known "stretched octaves", and also the typical tuning curve are caused by this. For plucked strings, stiffness forms character of sound, together with choice of plucking spot on string and with corpus. For bowed strings, it is preferable to have strings, with minor stiffness, because the interaction of bow/string is better. The effects of jitter and rippling (supplement of sound with statistic noise effects) are better formed by less stiff strings. For wound strings, minor stiffness is possible with cores of filament structure. Theory and examples will be discussed.

a) Present address: Erich Ohser Str. 10, D-08525 Plauen, Germany.

WEDNESDAY AFTERNOON, 17 MARCH 1999

ROOM H107, 4:55 TO 6:20 P.M.

\section{Session 3pNSa}

\section{Noise: Tribute to Henning von Gierke}

\author{
Richard L. McKinley, Cochair \\ Armstrong Laboratories, AL/CFBA, 2160 7th Street, Wright-Patterson AFB, Ohio 45433-7901, USA
}

Alice H. Suter, Cochair

Alice Suter and Associates, 575 Dogwood Way, Ashland, Oregon 97520, USA

Chair's Introduction-4:55

\section{Invited Papers}

5:00

3pNSa1. Henning E. von Gierke: Mentor, colleague, and public policy leader. Alice H. Suter (Alice Suter and Assoc., 575 Dogwood Way, Ashland, OR 97520) 
3pNSa3. The major historical events in the relating of noise-induced hearing loss to noise exposure. Daniel L. Johnson (Interactive Acoustics, 4719 Mile High Dr., Provo, UT 84604)

One event was the recognition of "boilermaker's disease." Another was some of the threshold shift studies during and after WWII that led to the BENOX report. After these developments, there were a series of key events and decisions that have led up to our current state of knowledge and procedures. How Henning von Gierke was influential in most of these and how his decisions have consistently proven to be correct will be demonstrated. For example, he contributed to the 1953 BENOX report, promoted the "equal energy concept," conceived and patented the first noise dosimeter, oversaw the EPA "levels" and criteria documents, and was the chair of the working group that wrote the current international standard (ISO-R1999) that relates noise exposure to noise-induced hearing loss. He was also instrumental in supporting many key research issues such as fetal noise exposure, asymptotic temporary threshold shift, and the longitudinal hearing of children. Henning's perseverance and dedication has provided society with an answer to one of his favorite questions, "how much noise is too much?"

6:00

3pNSa4. 50-year legacy of Henning E. von Gierke's scientific and engineering excellence. Richard L. McKinley (Armstrong Laboratories, AL/CFBA, 2160 7th Street, Wright-Patterson AFB, OH 45433-7901)

WEDNESDAY AFTERNOON, 17 MARCH 1999

ROOM H2032, 4:20 TO 6:20 P.M.

\title{
Session 3pNSb
}

\section{Noise: Rotor Noise from Helicoptors and Propellers}

\author{
Werner Dobrzynski, Cochair \\ DRL-Instiitut für Entwurfsaerodynamik, Lilienthalplatz 7, 38108 Braunschweig, Germany \\ Feri Farassat, Cochair \\ NASA Langley Research Center, Hampton, Virginia 23681-0001, USA
}

Invited Papers

3pNSb1. European helicopter rotor noise research-Highlights of recent projects. Wolf R. Splettstoesser (Deutsches Zentrum für Luft-und Raumfahrt e. V., Institut für Entwurfsaerodynamic, Lilienthal-platz 7, 38108 Branschweig, Germany, wolf.splettstoesser@dlr.de)

Significant advances in understanding, modeling, and controlling helicopter rotor noise have been made in the course of several major European rotor aeroacoustic research projects over the past years. The availability of the German-Dutch Wind Tunnel (DNW), in particular, has enabled research on relatively large main rotor models (up to typically $40 \%$ full scale). Here, the simultaneous application and use of blade-mounted absolute pressure sensors to study the very aeroacoustic source mechanisms, of advanced acoustic-data acquisition systems for the radiated noise, of non-intrusive flow visualization as well as flow and blade deflection measurement techniques to investigate the rotor wake and the blade dynamic response has provided much insight into the physics of rotor aeroacoustics, particularly into the rotor blade-vortex interaction (BVI) impulsive noise phenomenon. In efforts towards controlling BVI impulsive noise, in some research projects, active blade-root control techniques have been investigated to favorably affect the BVI geometry and thus minimize the impulsive noise radiation. Much of the experimental data acquired served as benchmark information to enhance the mathematical modeling of the rotor wake, the rotor airloads, and the noise radiation. In this lecture, several major European helicopter aeroacoustic research projects are discussed. 
3pNSb2. Analysis and capability of a noncompact integration method for rotor noise prediction. J. Prieur (ONERA/Dept. of Numerical Simulation and Aeroacoustics, 29 avenue de la division Leclerc, 92322 Chatillon Cedex, France)

Rotor noise computations starting from CFD input data using either the Kirchhoff or the generalized Ffowcs Williams-Hawkings surface integral methods in unsteady conditions may be very lengthy. This is the case for directivity studies or noise level contour calculations for which a high number of observer locations is required. To overcome this drawback, a fast integration method has been designed. It starts from the emission time which avoids having to solve the retarded time equation and uses directly as integration grid the CFD rotating grid (a priori not adapted to acoustic calculations). This is made possible thanks to a noncompact treatment of each grid element. The method has already been successfully applied in a transonic rotor case using indifferently a subsonically or supersonically rotating CFD grid for the surface integration (G. Rahier and J. Prieur, 53rd Forum of the American Helicopter Society, 29 April-1 May 1997). The method is made more understandable based on a new and clearer justification and its possibilities of extension are discussed.

\section{5:00}

3pNSb3. Analysis of the excess noise of aircraft pusher propellers. Johan B. H. M. Schulten (Aeroacoustics Dept., Natl. Aerosp. Lab. NLR, P.O. Box 153, 8300 AD Emmeloord, The Netherlands, schulten@nlr.nl)

A pusher propeller configuration offers several advantages over the traditional tractor arrangement. For instance, the wing of a twin-engined aircraft is no longer exposed to the swirling and turbulent slipstream. This results in a much smoother wing inflow, a prerequisite for aerodynamic refinement. Acoustically, the more aft position of the propellers considerably reduces the noise impact on the fuselage, yielding the potential of a low cabin noise level. However, in general, the exterior noise of pusher propellers is augmented by the interaction with impinging wakes and exhaust jets. The present paper addresses the physical mechanism of this interaction by applying an unsteady lifting surface theory to the propeller blades. This requires the incident field to be resolved in circumferentially harmonic Fourier components. For each component the unsteady blade pressure distribution is computed as source input for an acoustic calculation. From sample computations for typical operating conditions it appears that the interaction substantially contributes to the total radiated noise of pusher propellers. The present method offers the possibility to investigate in detail the effect of alterations in blade design and flight conditions on tonal level and directivity.

3pNSb4. Recent developments in helicopter rotor noise prediction at NASA. Kenneth S. Brentner (NASA Langley Res. Ctr., Hampton, VA 23681-0001, k.s.brentner@larc.nasa.gov)

Significant progress has been made recently toward the efficient and robust prediction of helicopter high-speed impulsive noise. The far-field approximation to the Ffowcs Williams-Hawkings (FW-H) quadrupole has been augmented with a rigorous mathematical development. Two new quadrupole formulations-a retarded-time formulation known as Q1A and an emission-surface formulation known as Q2-have been developed and implemented for subsonic and supersonic source motion, respectively. A key numerical advantage of the far-field approximation is that volume integration of the source region surrounding the rotor blades is only performed once in a preprocessing stage. The resulting quadrupole noise computation is reduced to surface integration over the rotor disk in the vicinity of the blades. The solution-adaptive marching-cubes integration algorithm used for the emission-surface formulation will be described. Another recent advance in helicopter noise prediction is the use of the FW-H equation on a permeable integration surface surrounding the source region. This application of the $\mathrm{FW}-\mathrm{H}$ equation has been shown to be both analytically and numerically superior to the Kirchhoff approach. Furthermore, numerical evidence is presented to demonstrate that the Kirchhoff method is inappropriate when a wake convects through the surface.

\section{Contributed Papers}

\section{5:40}

3pNSb5. Passive-active low-frequency vibroacoustical fault diagnosis method. Nadejda I. Bouraou (Dept. of Orientation and Navigation Systems, Natl. Tech. Univ. of Ukraine, 37 Peremogy pr., Kiev, 252056, Ukraine, nadya@burau.inec.kiev.ua), Leonid M. Gelman, and Pavel I. Marchuk (Natl. Tech. Univ. of Ukraine, Kiev, 252056, Ukraine)

The presented work is dedicated to the development of the new passive-active low-frequency automatic vibroacoustical early fault diagnosis method of helicopter engine units (compressor stages, turbine stages). The proposed method is based on signal processing of the rotating details of acoustic noise in the low-frequency range $(0-25 \mathrm{kHz})$. There are two important characteristics of this method: (a) it uses a nonstationary narrow-band vibration excitation with variable central frequency, and (b) this excitation is provided by rotating rotor, and user of the method will vary the central frequency, and (b) this excitation is provided by rotating rotor, and user of the method will vary the central frequency of rotor rotation. The basis for using this method is the fact that, at the presence of fault (for example, fatigue crack) in testing details, parameters of arising free and forced oscillations change (nonzero spectral amplitudes of higher harmonics). The new analytical dependencies of testing object oscillation parameters from the relative crack size and parameters of nonstationary excitation are received. A multidimensional testing data vector for diagnostics and evaluation of faults is proposed and investigated.
3pNSb6. An advanced field measurement system for rotorcraft flight noise. James C. Yu and Kenneth D. Wright (NASA LaRC, MS 235, Hampton, VA 23681-0001)

A Langley-developed field acoustic measurement system has been extensively employed to support the NASA aircraft and rotorcraft flight noise research programs since the early 1980s. The system was first successfully used in the NASA Forward Flight Effects on Turbofan Noise program. Starting from the late 1980s, driven by the increased demand on expanded measurement capabilities for rotorcraft flight noise, an advanced field measurement system development program was initiated to support the rotorcraft flight noise measurement, reduction, and prediction. The paper reviews the three major stages of this extensive development program: the analog measurement system stage, the digital measurement system stage, and the digital remote acquisition and storage system stage. The data processing technology associated with these different stages of the system will be presented. The paper will discuss the research drivers for the measurement system development, the development strategy used to meet the system requirement, and lessons learned at each stage of the development. Discussion will also be given on the future rotorcraft flight noise measurement challenges and the advanced measurement systems required to meet these challenges. 


\title{
Session 3pPA
}

\section{Physical Acoustics: Duct Acoustics II}

\author{
Walter Eversman, Cochair \\ Mechanical and Aerospace Engineering and Engineering Mechanics, University of Missouri, 202 Mechanical Engineering, \\ Rolla, Missouri 65401, USA \\ Dirk Ronneberger, Cochair \\ Drittes Physikalisches Institut, University of Göttingen, Burgerstrasse 42-44, D-37073 Göttingen, Germany
}

\section{Invited Papers}

3pPA1. Interaction between resonators and fluid motion in ducts. Dirk Ronneberger (Drittes Physikalisches Institut der Universitaet Goettingen, Buergerstrasse 42-44, D-37073 Goettingen, Germany, ro@ physik3.gwdg.de)

Each time one is confronted with unwanted narrow-band noise which propagates along a duct, it suggests itself to make use of resonators in the suppression of this noise. However, resonators may be highly sensitive to superimposed mean flow: Detuning and drastic decay of the quality factor is encountered in many cases, nevertheless, also the contrary may happen, namely the increase of the acoustic energy in the resonator. This may result in self-excited oscillations or in the amplification of the incident sound. The paper gives a review of these phenomena, and it will focus on the physical effects behind these phenomena. Simple models that are based on the governing physical effects have been developed and are compared to experimental results with different configurations of resonators in flow ducts. In the case of sound amplification the amplitude of the organized motion of the fluid may become very high so that the nonlinearity of the interaction with the mean flow becomes significant. The state of knowledge and the open questions in this respect will be addressed as well.

\section{4:40}

3pPA2. Active noise control by use of an aeroacoustic instability. Björn Lange and Dirk Ronneberger (III. Phys. Inst., Bürgerstr. 42-44, D-37073 Göttingen, Germany)

Ducts that are lined with a series of equally spaced cavities exhibit strong amplification of sound waves under specific flow conditions. The amplification is caused by an aeroacoustic instability wave traveling in the boundary layer of the lining. Since the effect is coupled with a drastic variation of the static pressure drop that depends on the amplitude of incident sound, the possibility is implied to efficiently generate high intensity sound at low frequencies: An incident sound wave with harmonically varying amplitude will cause a periodic modulation of the pressure drop and thus lead to the generation of sound at the modulation frequency. Based on these observations, an aeroacoustic loudspeaker has been designed combining a lined duct section as part of a flow channel and a conventional loudspeaker. The output power of the device amounts to up to $95 \mathrm{~dB}$ re: $1 \mathrm{pW} / \mathrm{Hz}$ at $\mathrm{Ma}=0.25$ for frequencies ranging between 0 and $200 \mathrm{~Hz}$ - an observation of some significance with regard to the fact that traditional loudspeakers tend to be rather inefficient at low frequencies. As a feasibility test, the aeroacoustic loudspeaker is used as an actuator in an active noise control setup. High attenuation of low-frequency noise is achieved.

\section{5:00}

3pPA3. Computational aeroacoustics of ducts using parallel computers. Lyle N. Long (Dept. of Aerosp. Eng., Penn State Univ., 233 Hammond Bldg., University Park, PA 16802)

This paper will discuss recent attempts at using computational aeroacoustics (CAA) algorithms on parallel computers to solve duct propagation problems. The algorithms are higher-order accurate methods for solving the Euler and Navier-Stokes equations. As computer speed increases, more and more of the physics can be simulated. This talk will discuss the current status and trends of parallel supercomputers. While hardware trends allow us to solve more complicated problems, algorithm developments are also essential. This talk will address some new methods for solving aeroacoustics problems. Most of these new developments involve hybrid schemes that couple CAA methods with integral methods or traditional CFD codes with CAA codes. Since aeroacoustics problems involve length and time scales that are beyond computer power at the present time, clever schemes must be developed for using appropriate algorithms for the various physical phenomena. One very promising scheme [J. Comput. Phys. 133 (1997)] is the nonlinear disturbance equation (NLDE) method, which uses a traditional CFD code to predict the steady-state portion of the problem, and then a higher-order method to solve for the disturbances. The steady-state portion is included in the coefficients and source terms in the NLDE. [Work supported by NASA Langley.] 
3pPA4. On the exact solution of the acoustic wave equation in a parabolic velocity profile in a hard-walled duct. L. M. B. C. Campos and J. M. G. S. Oliveira (Instituto Superior Téchnico, 1096 Lisboa Codex, Portugal)

The exact solution of the acoustic wave equation in a parabolic shear flow profile is obtained; the only exact solution given in the literature [Goldstein and Rice (1963); Jones (1977), (1978); Scott (1979); Koutsoyannis (1980)] is for a linear velocity profile, and the solution for the exponential shear flow is given elsewhere [Campos and Serrão (1998)]. The wave equation has three singularities, like the Gaussian hypergeometric equation, but it is of an extended type, since the singularity at infinity is irregular (all three singularities of the Gaussian hypergeometric equation are regular). The other two singularities of the present equation are regular, and one lies at the axis of the duct, and the other at the dicritical layers, where the Doppler-shifted frequency vanishes. The critical layers do not exist (they lie outside the duct) for longitudinal wave vector antiparallel to the mean flow (case I), i.e., propagation upstream in a local frame of reference. In the opposite case of longitudinal wave vector parallel to the mean flow (case II) there are three subcases, depending on whether the Doppler-shifted frequency on the axis of the duct is: (case II A) positive, i.e., critical layers are at imaginary "distance;"' (case II B) zero, i.e., the critical layer lies on the axis of the duct: (case II C) negative, i.e., two critical layers exist in the duct. In all cases (I, II A, II B, II C) it is possible to obtain the exact acoustic field over the whole flow region by expanding around the singularities and matching solutions. Since the acoustic wave equation in a shear flow does not lead to a Sturm-Liouville problem, the eigenfunctions need not be orthogonal or complete. There is a single set of natural frequencies and normal modes in cases I, II A, and II B, but not in case II C; in the latter case, where two critical layers lie in the flow, they may separate three sets of eigenvalues/ eigenfunctions, in the regions between by the critical layers and the walls.

5:40

3pPA5. Experimental verification of a radial mode analysis technique using wall-flush mounted sensors. Lars Enghardt (DLR-Institut fuer Antriebstechnik, Abteilung Turbulenzforschung Berlin, Mueller-Breslau-Str. 8, 10623 Berlin, Germany, lars.enghardt@dlr.de), Yanchang Zhang (LUK GmbH \& Co., 77815 Buehl, Germany), and Wolfgang Neise (DLR-Institut fuer Antriebstechnik, 10623 Berlin, Germany)

Sound fields in the inlet and outlet ducts of axial fans, compressors, and aircraft engines propagate as higher-order acoustical modes in a wide-frequency range. Decomposition of the sound field into azimuthal and radial modes permits direct conclusions on the sound generation processes and can lead to the identification of aerodynamic source areas. An established experimental method for assessing the sound field is to circumferentially traverse a radial microphone rake over 360 degrees, to measure the sound pressure in the duct at multiple circumferential and radial positions. On the inlet side of a turbo-machine this procedure cannot be employed because the wake of the rake would disturb the inlet flow conditions of the machine and alter its acoustic characteristics. Computer simulations have shown that the radial mode structure of the sound field can also be determined by measuring the sound pressure only at the inner duct wall, but at different axial locations. In this paper, experiments are described where the sound field in the outlet duct of an axial-flow fan is measured once with the conventional radial microphone rake and a second time with wall-flush mounted microphones. Subsequent radial mode analyses are carried out for both sets of measurement data to experimentally verify the new method.

\section{Contributed Paper}

6:00

3pPA6. Experimental study of acoustic impedance of a Helmholtz resonator submitted to a grazing flow. Slaheddine Frikha, Pierre Yves Hennion, and Samir Boukhari (Laboratoire de Mecanique Physique, UPMC CNRS, Upresa 7068, France, frikha@ccr.jussieu.fr)

Helmholtz resonators are usually used in ducts or in engines inlet and exhaust systems in order to reduce radiated noise. Their efficiency may be strongly affected since they are submitted to a fully turbulent grazing flow. An experimental analysis of a branched Helmholtz resonator composed by a circular neck and a circular cavity is presented here. An attempt is made to understand the evolution of entry impedance modification with respect to mean flow and the shear layer characteristics at the vicinity of the resonator aperture. An inverse method is used to identify the impedance of 25 resonators. This method presents the advantage that it is completely nonintrusive and does not require source control. The grazing flow modifies the orifice resistance and the effective neck length. A parametric study of these corrections is performed using experimental results obtained for a set of 25 resonators and a flow Mach number varying from 0 up to 0.25 . The mean flow structure is also analyzed using a numerical model. The dimensions of the tested resonators are similar to those usually used in the exhaust and inlet systems of automotive engines. That leads to a new parametric model of these corrections more suitable to engine applications. 


\title{
Contributed Poster
}

This poster will be on display in the Poster Gallery from Thursday to Friday, 18-19 March. Authors will be at their posters from 10:00 a.m. to 12:00 noon on Thursday, 18 March.

3pPA7. On the acoustic of inverse sinusoidal and catenoidal nozzles. L. M. B. C. Campos and F. J. P. Lau (Instituto Superior Técnico, 1096 Lisboa Codex, Portugal)

The acoustic wave equation, for quasi-one-dimensional propagation, along a duct of varying cross section, containing a low Mach number mean flow, is obtained using as variables either the potential or the velocity; the ray approximation, which holds only for wavelength short compared with the lengthscales or variation of cross section and mean flow velocity, is used as a factor to reduce the wave equation to a Schrödinger form. It is shown that the latter, reduced from, for the potential, is the most convenient to study the acoustics of catenoidal and sinusoidal nozzles; it is found that these inherit respectively the filtering properties of catenoidal horns, and transparency properties of sinusoidal horns. This approach also applies to the exponential nozzle whereas, for the Gaussian nozzle, the sound field can be expressed in terms of Hermite functions, using a semireduced form of the wave equations. The exact solutions of the nozzle wave equation, for the four families of ducts, are plotted as amplitude and phase versus distance, for several combinations of frequency and low Mach number. The nozzle families considered include the catenoidal in the sinusoidal and exponential and the first six eigenfunctions of Gaussian nozzles. The acoustics of inverse sinusoidal and catenoidal nozzles can also be studied, without use of the preceding transformations, via the solution of modified Mathieu equation.

\section{Session 3pPPa}

\section{Psychological and Physiological Acoustics: Perceptual Processing of Cross-Spectral Information}

\author{
John F. Culling, Chair \\ School of Psychology, Cardiff University, Cardiff CF1 3YG, UK
}

Chair's Introduction-4:15

Invited Papers

4:20

3pPPa1. Matching formant frequencies of complex sounds on different fundamentals. C. J. Darwin and Pascal Dissard (Exp. Psych., Univ. of Sussex, Brighton, Sussex BN1 9QG, UK)

In order to explore the ability of listeners to match formant frequencies in periodic sounds that differ in their fundamental frequency $(F 0)$, listeners adjusted the frequency of one formant of periodically excited one- or two-formant sounds to match the timbre of a similar target sound that could have either the same or a different $F 0$. Variability of matching (s.d. of matches within each listener) was low when matching sounds were on the same $F 0$. Variability increased when matching sounds had different $F 0$ s. However, this increase was larger for sounds with resolved harmonics than for those with unresolved harmonics. This difference may reflect the perceptual cost of estimating the spectral envelope (and formant frequency) from the excitation pattern. [Work supported by EPSRC Grant No. GR/L03422.]

$4: 40$

3pPPa2. A "missing-data" model of $\boldsymbol{F}$ 0-invariance in vowel perception. Alain de Cheveigne (LLF, Univ. of Paris 7, 2 Pl. Jussieu, Paris, France)

In steady-state voiced speech production, the vocal tract transfer function is sampled at multiples of the fundamental frequency $(F 0)$. At high $F 0$, sparse sampling causes two problems: (a) a gradual loss of information defining the spectral shape, and (b) $F 0$-dependent distortion due to aliasing. If the shape of the spectral envelope contains lag-domain components (spatial frequencies) beyond the Nyquist limit, they are folded relative to the Nyquist limit and mixed with in-band components. The Nyquist limit ( $T 0 / 2$, where $T 0=1 / F 0$ ) depends on the spacing between sampling points along the spectral envelope, and thus on $F 0$. Distortion is thus $F 0$-dependent, and all the more severe as $F 0$ is high. Smoothing or interpolation are ineffective in dealing with this problem, and cannot produce an $F 0$-invariant pattern. A solution is proposed based on the concept of "missing feature theory," recently proposed for speech recognition. Pattern matching is limited to available data ( $F 0$-spaced sample points) using an $F 0$-dependent weighting function. All other points are ignored. The model is proposed in two versions, one that operates on the short-term spectrum or excitation pattern, the other on the autocorrelation function. The model ensures $F 0$-independent vowel identification, at the cost of an $F 0$ estimate. 
3pPPa3. Evidence of a dissociation between the mechanisms governing the perceptual grouping of spectral components and the computation of low pitch. Brian Roberts and Jeffrey M. Brunstrom (School of Psych., Univ. of Birmingham, Edgbaston, Birmingham B15 2TT, UK, b.roberts@bham.ac.uk)

The perceptual fusion of harmonically related partials is often assumed to result from the operation of a template mechanism that is responsible for computing low pitch. This dual-role hypothesis has been tested using frequency-shifted complexes. These sounds are inharmonic, but preserve a regular spectral pattern of equal component spacing. Stimuli had a nominal fundamental $(F 0)$ frequency of $200 \mathrm{~Hz}$ (roving range $\pm 20 \%$ ), and were frequency shifted either by $25.0 \%$ or $37.5 \%$. Three consecutive components (6-8) were removed and replaced with a sinusoidal probe, located at one of a set of regularly spaced positions spanning the gap. On any trial, subjects heard a complex tone followed by an adjustable pure tone in a continuous loop. Subjects were well able to match the pitch of the probe unless it corresponded with a position predicted by the spectral pattern of the complex. In contrast, hit rates were not depressed for probes positioned at integer multiples of the $F 0$ (s) corresponding to the low pitch(es) of the complex, which were predicted from previous data [Patterson, J. Acoust. Soc. Am. 53, 1565-1572 (1973)]. The findings suggest that separate mechanisms are responsible for the computation of low pitch and for the perceptual grouping of partials.

\section{$5: 20$}

3pPPa4. Auditory processing of coherent and incoherent frequency modulation. Robert P. Carlyon (MRC Cognition and Brain Sci. Unit, 15 Chaucer Rd., Cambridge CB2 2EF, England, bob.carlyon@mrc-cbu.cam.ac.uk)

When two elements of a visual array move coherently, there is a strong tendency to group them perceptually. This has prompted several authors to suggest that acoustic frequency components which undergo coherent FM should be perceptually fused, and that those which are modulated incoherently should be segregated. This paper will review the mounting body of evidence demonstrating that, once a number of co-varying cues are controlled for, the auditory system is insensitive to the difference between coherent and incoherent FM. Such cues include the greater inharmonicity, smaller swings in average frequency, and more salient distortion products which can occur with incoherent compared to coherent modulation. In addition, new evidence will be presented showing that listeners can be more sensitive to one form of incoherent FM than to another: Opposite-direction quasi-linear frequency sweeps imposed on two carriers are more detectable when the frequency of the lower component decreases and that of the higher component increases than vice versa. This occurs even though the frequency sweeps imposed on the individual components are equally detectable when those components are presented in isolation, and even though within-channel interactions are masked by noise.

\section{Contributed Papers}

$5: 40$

3pPPa5. Searching for a time window for timbre: Dynamic spectral profiles. M. E. H. Schouten and W. J. M. Peeters (Res. Inst. for Lang. and Speech UiL-OTS, Utrecht Univ., Trans 10, 3512 JK Utrecht, The Netherlands, bert.schouten@let.uu.nl)

Subjects were asked to match resonance glides, of various durations and sweep widths around $1000 \mathrm{~Hz}$, with two connected steady-state resonances whose frequencies could be controlled independently. The expectation was that short resonance glides (below $100 \mathrm{~ms}$ ), would give rise to single, apparently stationary, perceptual events, so that the two matching steady-state patterns would not rise or fall. Long glides, on the other hand, were expected to be perceived as rising or falling and to be matched accordingly. It was hoped that this would provide a basis for an explanation of diphthongs as unitary percepts. The resonances formed part of dynamic spectral profiles and consisted of single formantlike peaks swept along the top of a spectral base, consisting of 25 harmonically or logarithmically spaced components from 200 to $5000 \mathrm{~Hz}$. There was an effect of duration, although it interacted with sweep width. At durations shorter than about $120 \mathrm{~ms}$, subjects placed the two steady profiles that they had to match with the dynamic profile, much closer together than with durations over $120 \mathrm{~ms}$; however, this only occurred if sweep width was more than $600 \mathrm{~Hz}$.

\section{6:00}

3pPPa6. A spectral layer model in speech and music perception. Werner A. Deutsch (Austrian Acad. of Sci., Liebiggasse 5, A-1010, Vienna, Austria,wad@kfs.oeaw.ac.at)

According to a model of the peripheral hearing system establishing a masking and overmasking paradigm, speech and music signals have been split into several spectral layers by means of spectral subtraction. Spectral components below the so-called psychoacoustical irrelevance threshold are eliminated in a first processing step. The resulting signal, which exhibits no noticeable difference to the unprocessed one, is fed into an overmasking procedure applying flattened hearing masking functions. This action implements a kind of a spectral peak detector device. Consequently, the discrimination between spectral peaks (auditory figure) and background components, especially in reverberant environments, is improved. As a result, different audible signal parts according to different spectral layers are obtained, separating spectral peaks and weaker spectral components. In a certain range, both parts of speech and music signals are equally intelligible. In music, leading voices can be extracted and separated from the orchestra sound. Recent research indicates that this type of figure-background discrimination based on the dynamic characteristics of the acoustic signal only can exhibit several perceptually relevant features in listeners with normal hearing as well as in subjects with sensineural hearing impairment. 


\title{
Session 3pPPb
}

\section{Psychological and Physiological Acoustics: Sound Quality}

\author{
Gerhard Krump, Chair \\ Harman Audio Electronic Systems GmbH, Schlesische Strasse 135, D-94315 Straubing, Germany
}

\section{Contributed Papers}

$4: 20$

3pPPb1. Interior car sound quality-Assessment of acceleration noises. Reinhard Weber (Dept. of Phys., Carl-von-Ossietzky Univ., D-26111 Oldenburg, Germany)

Investigation of booming noise effects was the reason for the present study. Interior noises of six different cars accelerating from 1500 to 6000 rpm at third gear (full load) were presented over headphones. Seventy normal-hearing subjects (approximately one-third males and two-thirds females) aged from 20 to 60 years (at least 15 subjects per decade) took part in two experiments. After an initial orientation phase, preference judgments were made using a paired comparison paradigm. In experiment 2, the noises were assessed on a bipolar categorical scale (disagreeable) agreeable) with nine subdivisions. Immediately after the experiments, an interview was conducted to learn about the subjects' impressions about the noises. The preference data were analyzed using MDPREF and compared to the categorical judgment data for different subsets of subjects. The results on subjective data will be presented, together with a correlation analysis on their relationship with a number of acoustical and psychoacoustical signal parameters (level, loudness, sharpness, fluctuation strength, roughness).

\section{4:40}

3pPPb2. The influence of sound quality on expected visual impression. Monica M. Söderholm (Electrolux Resesearch \& Innovation, $\quad \mathrm{S}-105 \quad 45 \quad$ Stockholm, monica.soderholm@notes.electrolux.se) and Daniel Västfjäll (Chalmers Univ. of Technology, S-412 96 Göteborg, Sweden)

Information from visual and auditory systems is often linked. One example of the cross-modal connection between sound and visual experience is different materials that easily can be identified by their sound quality. The purpose of this investigation was to analyze the anticipated visual impression of three vacuum cleaners when listening to the product. The participants in the listening test where asked to describe the visual impression of the vacuum cleaners and also how they thought the vacuum cleaner sounded like with own words. The 24 listeners were both female and male of different ages and the vacuum cleaners were judged live. Often something that is large, for instance, a truck compared to a car, does produce more sound. The experience that large products are louder than small products is an influence when listening to vacuum cleaners. The listeners expected a large vacuum cleaner when listening to a rather loud vacuum cleaner. There was a unanimous opinion among the participants that a loud vacuum cleaner was ugly, had a box shape, and was old. The vacuum cleaners that were described to have a nice sound were described as small and round with a nice design and nice colors.
5:00

3pPPb3. Comparison of two semantic-differential test methods. Etienne Parizet (Renault, 67 rue des bons raisins, 92508 Rueil-Malmaison Cedex 9, France, etienne.parizet@renault.com)

Two methods for conducting a multidimensional test were investigated. In the first and classical one, a sound (or a pair of sounds) is presented to the subject, who has to evaluate it (or compare the two sounds) for the various semantic parameters. In the second one, the test is repeated for each parameter (i.e., subjects must evaluate the whole set of sounds for the first parameter, then again for the second one, and so on). These two methods were compared on the basis of five diesel engine noises in small cars driving at idle. Sounds were compared in pairs for seven parameters (pleasant, loud, etc.). The characterization of sounds thus obtained was similar for the two methods. The second one proved to be more accurate (the accuracy is evaluated on the basis of repetition and triadic errors) and with a shorter duration than the first one, because listeners are concentrated on the selected sound parameter while hearing a new stimulus. Therefore, this method is recommended for conducting semantic differential tests.

\section{$5: 20$}

3pPPb4. About perceptivity of narrow-band frequency response variations of loudspeakers. Gerhard Krump (Harman Audio Electron. Systems GmbH, Schlesische Str. 135, D-94315 Straubing, Germany, gkrump@harman.de)

Many loudspeakers show peaks and gaps at $1 \mathrm{kHz}$ caused by the connection of the hard cone and soft surround, which produces interferences and complex vibrational modes. Great efforts are made by the speaker manufacturer to avoid this by changing the surround geometry and material, whereas primarily knowledge about the perceptive limits of the peaks and gaps in frequency response would be necessary. For this investigation line spectra with equal amplitudes and statistical phases between 1 $\mathrm{Hz}$ and $20 \mathrm{kHz}$ were calculated by a personal computer. At $1 \mathrm{kHz}$ spectral enhancements or gaps with a bandwidth of $1 \mathrm{~Hz}$ or of a half, one, or two critical bands were realized with five different critical band levels. Ten test persons had to compare the first line spectrum with equal amplitudes (anchor) with the second signal with spectral changes in a yes-no procedure presented by an earphone with free-field equalizer at a SPL of $70 \mathrm{~dB}$. The just-noticeable changes in spectral envelope are different for enhancements and gaps and depend on the bandwidth and the critical band level of the variations. They can be explained with calculated masking patterns and literature data. 
3pPPb5. Determination of hearing properties and their connection to psychoacoustical parameters. Kai Johannsen and Holger Prante (Inst. of Tech. Acoust., Einsteinufer 25, 10587 Berlin, Germany, johannsen@mach.ut.tu-berlin.de)

The sound quality of products is often evaluated in hearing experiments using the semantic differential and a subsequent factorial analysis. In this study the method is applied to determine basic hearing properties. In order to achieve optimal results of the hearing experiment, special interest has been taken in the choice of adjectives and sounds. This selection is based on the results of a previously conducted survey of sound attributes. The selected 25 sounds were recorded using an artificial head and presented binaurally to a jury of 20 adults with normal hearing. A factorial analysis of the ratings on 24 attribute scales produced six factors representing basic perceptive cognitive and emotional hearing dimensions. In a last step connections between these dimensions and psychoacoustical parameters calculated from the sounds were evaluated using an analysis of correlation. Although the results show high correlations between loudness, sharpness, and pitch strength and equivalent attributes, no such connections could be found for the psychoacoustical roughness and fluctuation strength. In order to find better-correlated parameters, other calculation methods for these properties are investigated.
3pPPb6. New approach in measuring auditory source width. Jörg Becker, Markus Sapp, and Frederik Görges (Inst. of Commun. Eng., Aachen Univ. of Technologie, 52056 Aachen, Germany)

Depending on the acoustical surrounding sound sources appear to be broadened. This spatial impression can be rated by a quantity called auditory source width. In this paper a new method for determining this auditory source width is presented. The system is based on the reproduction of an artificial head recording of the examined sound field mixed with a pair of virtual acoustic pointers. These pointers can be moved by test persons to fit the left and right border of the sound source whose ASW has to be rated. The acoustical pointers are implemented as digital signal generators followed by real-time HRTF filters, measured in azimuth steps of $2 \mathrm{deg}$ and cross faded between the directions. Investigations on suitable temporal and spectral shapes of the pointer signals have been made to minimize the localization blur of the pointer. The HRTF measurements and the recordings were done with different artificial heads in order to examine their influence on the determined ASW. Furthermore the influence of different sound field reproduction systems-headphone and crosstalk cancellation-are examined.

WEDNESDAY AFTERNOON, 17 MARCH 1999

ROOM H2036, 4:20 TO 6:20 P.M.

\title{
Session 3pSA
}

\section{Structural Acoustics and Vibration: Structural Vibrations}

\author{
Jacek Jarzynski, Cochair \\ George W. Woodruff School of Mechanical Engineering, Georgia Institute of Technology, Atlanta, Georgia 30332-0405, USA \\ David Feit, Cochair \\ Carderock Division, Naval Surface Warfare Center, 9500 MacArthur Boulevard, West Bethesda, Maryland 20817, USA
}

\section{Contributed Papers}

4:20

3pSA1. Torsional waves in a viscoelastic layer. Qingchun $\mathrm{Li}$ and Jacek Jarzynski (The George W. Woodruff School of Mech. Eng., Georgia Inst. of Technol., Atlanta, GA 30332-0405, jacek.jarzynski@me.gatech.edu)

The objectives of this study are first to examine experimentally the propagation of torsional waves in a viscoelastic material, and second to use these data to determine the complex shear modulus of the material. The viscoelastic material used in this study is neoprene rubber. A magnetic drive is used to generate a transient, one-cycle, torsional wave in a brass rod, and this wave is transmitted into a layer of neoprene rubber bonded to the rod. A fiber-optic laser Doppler vibrometer is used as a noncontact probe to measure the torsional wave transmitted through the neoprene layer. The experimental data are compared with predictions from a theoretical model [D. P. Thomas, Int. J. Eng. Sci. 6, 565-570 (1968)]. Also, the complex shear modulus is determined from a least-square fit of the theoretical model to the experimental data using the downhill Simplex method. Another application of torsional waves is to nondestructive testing of materials, particularly for testing the strength of adhesive bonds between layers. [Work supported by ONR.]
4:40

3pSA2. Estimates of dynamic strain and stress in pipes by measured average vibration velocity. Svante Finnveden (Dept. of Vehicle Eng., KTH, SE-100 44 Stockholm, Sweden, svantef@fkt.kth.se) and Roger Pinnington (Univ. of Southampton, Southampton SO17 1BJ, England)

A velocity method for estimating dynamic strain and stress in pipe structures is investigated. With this method predicted or measured spatial average vibration velocity and theoretically derived strain factors are used to estimate maximum strain. The nondimensional strain factor is defined as the maximum strain times the ratio of the sound velocity to the spatial rms vibration velocity. Measurements are made confirming that this is a descriptive number. Using a spectral finite-element method, numerical experiments are made varying the pipe parameters and considering all 16 possible homogeneous boundary conditions. While indicating possible limitations of the method when equipment is mounted on pipes, the experiments verify the theoretical results. The velocity method may become useful in engineering practice for assessments of fatigue life. 
3pSA3. On the dynamic interaction of a pipe conveying fluid with an elastic resisting medium. Ventzeslav A. Dzhupanov, Peter A. Djondjorov, and Vassil M. Vassilev (Inst. of Mech., Bulgarian Acad. of Sci., Sofia, Bulgaria)

The problem of the dynamic stability of pipes conveying high-speed fluid and lying on an elastic foundation has been a subject of significant interest during the past 30 years due to the broad application of pipes as structural components in different technologies. The present study contributes to the solution of this problem, suggesting a new model of the foundation - an infinitely deep elastic stratum, whose width is equal to the length of the attached pipe. The material of the foundation is supposed to be a homogeneous and isotropic one. Its dynamic behavior is considered in the context of linear elasticity. The pipe vibration is governed by the usual Bernoulli-Navier-Mohr theory. An approximate solution of the respective mathematical problem is obtained applying Galerkin's method. As a result, the influence of the medium physical characteristics to the flutter dynamic stability of cantilevered pipes through the pair of acoustic velocities is examined.

\section{$5: 20$}

3pSA4. Investigation at a top counter weight of a washing machine. Ulrich Heise (Noiselab, FHP Motors GmbH, Klingenbergplatz 1, 26133 Oldenburg, Germany) and Wolfgang Harbich (Noiselab, 13599 Berlin, Germany)

During the development of a new washing machine a problem came up in spite of the durability of the newly designed top counter weight. The top counter weight is necessary to balance the drum of the machine. The problem was caused by the dynamical behavior of the system. A modal analysis was made at the compensation weight in the machine. Several different modes and eigenvalues were detected. The disturbing mode was found. It was caused by the stiffness of the fastening and the mass of the weight. The top counter weight was fastened in the axial direction of the drum. The disturbing mode was eliminated due to the change of fastening from axial to radial fastening. The problem is now solved. Another point of view are the other eigenvalues of the weight. The size of the weight looks like a xylophone bar and, in the same manner, it can vibrate. These modes of the weight itself are not disturbing modes, because the excitation forces are too small in this area and at this frequency range for exciting the compensation weight.
3pSA5. A mixed use of finite element and geometrical techniques. Philippe Jean (Centre Scientifique et Technique du Bâtiment, 24 rue Joseph Fourier, 38400 St. Martin d'Hères, France)

The finite-element method is limited to either small geometries or to the low-frequency range, whereas geometrical approaches are best applied at medium and high frequencies, but do not take vibrating surfaces into account. When the vibrating boundaries of a volume are only a small part of the total boundary, it is worth combining the two methods. By using geometrical methods to compute a Green's function that includes all wave reflections on the surfaces of the volume, it is possible to restrict the integral representation of the pressure field to the vibrating surfaces. The surfaces to be meshed will be reduced to surfaces with known velocity fields. This approach offers a means of reducing computation times. It has been applied to large volumes corresponding to a room with a vibrating concrete wall and to a train with a vibrating window.

\section{6:00}

3pSA6. Irregular modes at the endshield of a heating system motor. Ulrich Heise (Noiselab, FHP Motors GmbH, Klingenbergplatz 1, 26133 Oldenburg, Germany)

Nowadays, more and more modern heating systems are not installed in separated rooms, so the expectation of noise comfort has been increased for the last years. A noise and vibration optimization was made at an electric motors b-sided endshield. Sometimes, the old endshield emitted airborne noise. This was caused by the structure-borne noise of the bearings together with a mode of the endshield. A modal analysis was made to detect the disturbing mode. The amazing thing of this test was that the $(0,1)$ mode frequency has a higher resonance frequency as the $(1,1)$ mode. Normally the $(1,1)$ mode frequency has a 1.594 higher frequency than the $(0,1)$ mode resonance [N. H. Fletcher and T. D. Rossing, The Physics of Musical Instruments (1991), pp. 69-71]. The b-sided bearings can make a mass coupling from the rotor to the endshield in radial direction, but not in this way in the axial direction. The $(1,1)$ mode of the endshield had nearly the same structure-borne amplitude as the $(0,1)$ mode, but the $(1,1)$ mode does not transmit airborne noise. This depends on the mechanical dimensions of the $(1,1)$ mode, because this mode is working with its dimensions on an acoustical short circuit on air. 


\title{
Session 3pSC
}

\section{Speech Communication: Psychoacoustic Methods in Speech Recognition II}

\author{
Birger Kollmeier, Cochair \\ AG Medizinische Physik, Universität Oldenburg, Postfach 2503, D-26111 Oldenburg, Germany \\ Hynek Hermansky, Cochair \\ Oregon Graduate Institute of Science and Technology, Beaverton, Oregon 97006-8921, USA
}

Chair's Introduction-4:15

Contributed Papers

4:20

3pSC1. Application of a roughness model to pathological voices. Robert Reuter (Inst. Electron., Techn. Univ., Einsteinufer 17, D-10587 Berlin, Germany, rreuter@tubife1.ee.tu-berlin.de) and Hanspeter Herzel (Humboldt Univ., D-10115 Berlin, Germany)

Irregularities in voiced speech are often related to the intrinsic nonlinearities in the vibrations of the vocal folds. Desynchronization of vibratory modes can induce nonlinear phenomena such as subharmonics, biphonation (two independent pitches), and deterministic chaos. The resulting complex signals exhibit various amplitude and frequency modulations and are, therefore, perceived typically as rough voices. In clinical studies of pathological voices perceptive roughness scores are widely used. So far, however, no satisfactory correlation of ratings of roughness and acoustic parameters has been found. In this presentation a modified Aures model for the quantification of roughness is introduced. It is exemplified that subharmonics lead to high roughness scores whereas the roughness of biphonation depends strongly on the two frequencies. Finally, a set of 120 perceptually evaluated pathological voices is studied. It turns out that the roughness score obtained from the psychoacoustic model is stronger correlated to the ratings of phoniatricians than conventional perturbation measures such as jitter and shimmer.

\section{$4: 40$}

3pSC2. How children recognize spoken words in speech recognition threshold assessment. Lebel Caroline (Institut Raymond-Dewar, 3600 Berri, Montreal, QC H2L 4G9, Canada) and Picard Michel (Universite de Montreal, Montreal, QC H3C 3J7, Canada)

The purpose of this study was to investigate the potential influence of language context effects on the speech recognition threshold (SRT) in children of different ages. To determine the respective contributions of audibility and linguistic factors to the SRT, the speech intelligibility index (SII), and indices $k$ and $j$ as proposed by Nittrouer and Boothroyd [J. Acoust. Soc. Am. 87, 2705-2715 (1990)] were calculated for the following conditions: (1) SRT using an open-set response format; (2) SRT using a closed-set response format (dissimilar foils to increase predictability); and (3) SRT using test words at the beginning of a short sentence. Listeners were three groups of 24 normally hearing children in the first, third, and fifth grades, respectively. Results indicated that SII and factor $j$ decreased significantly and factor $k$ increased proportionally as a function of both lexical and sentence-level context effects in all groups. This suggests that audibility factors are less critical in the facilitating presence of language contexts. The largest effect was shown for lexical context effects with predictable outcome of decreased SRTs. These findings are compelling evidence of language context effects playing a significant role in school-age children required to recognize speech at barely audible levels.
5:00

3pSC3. Silicon cochlea: A digital VLSI implementation of a quantitative model of the auditory system. Matthias Brucke, Wofgang Nebel (Univ. of Oldenburg, Computer Sci. Dept., VLSI Group, 2611 Oldenburg, Germany), Alexander Schwarz, Bärbel Mertsching (Univ. of Hamburg, Hamburg, Germany), Martin Hansen, and Birger Kollmeier (Univ. of Oldenburg, Oldenburg, Germany)

In this paper a digital VLSI implementation of a quantitative model of the auditory system is presented, which includes several processing stages physiologically and psychoacoustically motivated by the function of the human ear. The model was successfully applied to a wide range of applications from psychoacoustical experiments to speech recognition. The hardware design is derived from a $\mathrm{C} / \mathrm{C}++$ notation of the algorithms using floating point arithmetics. One application of the model is used to determine the necessary internal precision of the computations for a transfer into a version suitable for a hardware implementation using fixpoint arithmetics. This application provides a very valid test bench since the significant speech processing features of the model are proofed by computing an objective speech quality measure. The processing scheme is divided into designs for two FPGAs and will be converted into ASICs in a later version. The implementation of the model as dedicated hardware provides efficient coprocessing power and allows implementations of complex auditory-based speech processing algorithms in real time because the chipset performs substantial parts of the necessary calculations.

$5: 20$

3pSC4. Entropy rate based speech segmentation. Wolfgang Wokurek (Inst. of Natural Lang. Processing, Exp. Phonet., Stuttgart Univ., Azenbergstrasse 12, Stuttgart, 70174, Germany, wokurek@ims.uni-stuttgart.de)

The sequence of estimates of the speech signal's entropy rate is investigated as a potential basis for speech segmentation. Raising and falling edges of that entropy rate curve and its maxima and minima are considered as candidates for segment boundaries. These prominent points are compared to the phonetic segment boundaries and to acoustic landmarks [S. A. Liu, J. Acoust. Soc. Am. 100, 3417-3430 (1996)]. The comparison is made using the "Kiel corpus of read speech" which is a phonetically manually labeled corpus of German utterances. The evaluation considers the entropy rate labels against the background of the phone segments. In particular the position and the count of the entropy rate labels within each phone are appraised. The average results are reported both as totals and broken down into phone classes and phones. The frame duration of the entropy rate estimation controls the smoothness of the curve and hence the label count per phone. A similar evaluation relates the entropy rate labels to the acoustic landmarks. 
Session 3pSP

\title{
Signal Processing in Acoustics and Engineering Acoustics: Binaural Technology II
}

\author{
Mahlon Burkhard, Cochair \\ Sonic Perceptions, Inc., 6964 Kennsington Road, Brighton, Michigan 48116, USA \\ Jens Blauert, Cochair \\ Communication Acoustics, Ruhr-Universität Bochum, D-44780 Bochum, Germany \\ Chair's Introduction-4:35
}

Contributed Papers

\begin{abstract}
4:40
3pSP1. Implementation issues in the development of a real-time, Windows-based system to study spatial hearing. Joel D. Miller (Raytheon, NASA Ames Res. Ctr., M.S. 262-6, Moffett Field, CA 94035-1000, jdmiller@mail.arc.nasa.gov), Jonathan S. Abel (San Jose State Univ., NASA Ames Res. Ctr., Moffett Field, CA 94035-1000), and Elizabeth M. Wenzel (NASA Ames Res. Ctr., Moffett Field, CA 94035-1000)
\end{abstract}

As a tool to study spatial hearing, a customizable real-time 3-D-audio and virtual acoustic environment rendering system, Sound Laboratory (SLab), was developed. Design goals for this system included flexibility, extensibility, maintainability, and a fast development cycle per experiment. SLab is a Windows application composed of five distinct software layers: Application programming interface (API), 3D projection, signal flow translation, signal routing and processing, and digital signal processing library. The acoustic scene, including listener, source, and environment characteristics, is specified in the API layer and translated in the 3-D projection layer to geometric quantities, such as range and arrival angle, for each path rendered between the source and listener. The geometry and head-related transfer function coefficients are rendered using a set of parallel finite impulse response filters and delay lines built on Intel's Signal Processing Library. This rendering architecture provides the flexibility and the extensibility required for psychoacoustic experimentation, while the modular nature of the programming interface ensures maintainability and quick development of new experiments. The psychoacoustic parameters to be manipulated, the signal flow diagram, and the five software layers are discussed in detail, and various implementation issues are examined.

\section{5:00}

3pSP2. Computational model of an individual head-related transfer function using the BEM. Brian F. G. Katz ${ }^{\mathrm{a}}$ (Acoust. Dept., Penn State Univ., State College, PA 16801)

The head-related transfer function (HRTF), determined by the complex geometry of the pinnae and head, is the primary research and commercial tool for exploring and exploiting 3-D auralization and auditory localization. Research into the determining characteristics of the HRTF is difficult, as the anatomical variations between individuals are many. These variations make comparisons between individuals less than ideal for determining the relation between structure of the individual and components of the HRTF. This paper will discuss an investigation into the use of a geometrical model of an individual to calculate the HRTF. The calculation utilizes the boundary element method (BEM). As a tool, the use of a computer model allows for selected variations in the individual's anatomy and direct observation of the results, a difficult task in experimental mea- sures. Previous work by the author discussed the variability in experimental measurement techniques of the HRTF. Comparisons are made between the model results and these HRTF measurements for the same individual. Some preliminary variations to the model and their effects are also discussed. Computational results show good agreement with the general trends of the measurements and the frequency limitations of the computation. ${ }^{\text {a) }}$ Currently at Artec Consultants, Inc., 114 W. 26th St., New York, NY 10001

$5: 20$

3pSP3. Auditory perception of reverberant surroundings. Jesper Sandvad (Waisman Ctr., Univ. of Wisconsin, Madison, WI and Acoust. Lab., Aalborg Univ., Denmark)

Three experiments on perception of reverberant surroundings were performed. In the first experiment, listeners were presented with binaural recordings of a speech signal and a set of photographs taken in different rooms, and were asked to point out where each recording was made. Most listeners performed very well in this task (average number of correct answers was 70\%), showing that reverberation contains information on room properties that the listeners are able to extract. In the second experiment, listeners were asked to judge the relative size of different rooms by listening to the recorded signals. The results show substantial individual differences, with some listeners being close to veridical performance while others had only a few correct answers. A scaling analysis suggests that some listeners use the direct/reverberant energy ratio, while others use reverberation time as a cue for room size. In the third experiment, artificial reverberation generated by a geometric room model (including individual reflections) and from a stochastic model (controlling only energy measures) were compared with signals generated directly from measured room impulse responses. The results show comparable performance, suggesting that energy measures are the most important for assessing information on room parameters. [Work supported by ONR Grant No. N00014-96-10675.]

\section{$5: 40$}

3pSP4. Comparison of wideband LMS, subband LMS, and a nonlinear entropic neural approach to adaptive noise cancellation for speech enhancement. P. W. Shields, M. Girolami, and D. R. Campbell (Dept. of Electron. Eng. and Phys., Univ. of Paisley, High St. Paisley, Renfrewshire PA1 2BE, Scotland, paul@diana22.pailsey.ac.uk)

An empirical comparison of three adaptive algorithms for speech enhancement in noisy reverberant conditions is presented. The subband least mean square (LMS) [E. Toner and D. R. Campbell, Speech Commun. 12, 
253-259 (1993)] and minimum entropy noise reduction schemes [M. Girolami, Electron. Lett. 33(17), 1437-1438 (1997)] are compared with a reference wideband LMS approach [S. D. Stearns and R. A. David, Signal Processing Algorithms (Prentice-Hall, Englewood Cliffs, NJ, 1988)]. All three methods were applied to the enhancement of speech corrupted with speech-shaped noise. The schemes were tested using simulated anechoic and real-room reverberant $\left(T_{60}=0.3 \mathrm{~s}\right)$ environments. The anechoic and real-room recordings were binaural including head shadow and diffraction effects. The subband LMS adaptive processing scheme uses the LMS adaptive noise cancellation algorithm in frequency-limited subbands. The inputs from each microphone are split into 16 contiguous subbands using a cochlear distribution according the function provided by Greenwood [J. Acoust. Soc. Am. 87, 2592-2605 (1990)]. Each frequency-limited subband is processed using a LMS adaptive noise cancellation filter operating in an intermittent or continuous mode depending on input signal characteristics. The neural network approach is motivated by temporally sensitive hebbian super-synapses, which may form sparse representations based on commonly occurring spatio-temporal input patterns. The results for real-room signals demonstrate statistically equivalent performance of the subband LMS and wideband neural method, both of which prove superior to the wideband LMS algorithm.
6:00

3pSP5. Evaluation of the air particle velocity signal from the binaural pressure impulse response. Domenico Stanzial (CIARM-CNR c/o Cemoter Acoust. Dept., Via Canal Bianco 28, I-44044 Cassana, Ferrara, Italy) and Davide Bonsi (CIARM c/o Univ. of Ferrara, I-44100 Ferrara, Italy)

From the Lagrangian theory in linear acoustics one clearly infers that the generalized momenta of the sound field are the acoustic pressure and the three components of the air particle velocity. This means that only through the knowledge of the full set of these four quantities a complete physical identification of the sound field can be achieved. The binaural technology represents an important tool for this goal, since it offers a method for reconstructing the pressure sound field by means of a couple of pressure impulse responses measured in two close points. An additional improvement of the binaural signal processing can be obtained using the two pressure input signals for evaluating the velocity component along the direction determined by the measurement points. First the velocity impulse response component is evaluated by means of the Euler equation and then, by convolving this signal with the usual anechoic signal, the velocity is reconstructed. In this paper the rigorous mathematical procedure for accomplishing the above task is presented.

WEDNESDAY AFTERNOON, 17 MARCH 1999

ROOM H104, 4:40 TO 6:20 P.M.

\title{
Session 3pUWa
}

\section{Underwater Acoustics: Sediment Acoustics}

\author{
F. Michael Pestorius, Cochair \\ Applied Research Laboratories, University of Texas, Austin, Texas 78713-8029, USA \\ Nicholas G. Pace, Cochair \\ SACLANT Undersea Research Centre, Viale S. Bartolomeo 400, 19038 La Spezia, Italy
}

Contributed Papers

\section{4:40}

3pUWa1. Volume scattering realizations from ocean sediment soundspeed and density inhomogeneities. Kevin D. LePage (SACLANT Undersea Res. Ctr., Vl. S. Bartolomeo 400, 19138 La Spezia, Italy) and Henrik Schmidt (MIT, Cambridge, MA 02139)

Synthetic scattered field calculations can serve as a useful tool for evaluating the practicality of estimating bottom scattering properties from in-situ measurements. Here the temporal and angular characteristics of early time reverberation from volume inhomogeneity scatterers in the subbottom are investigated using perturbation theory in the framework of a spectral integral approach. In order to model realistic sediments more accurately, the theory treats the scattering from both sound-speed and density fluctuations in the presence of a background sound-speed gradient. To allow the simulation of realistic experimental geometries, the scattered field is propagated to various receivers through a general multilayered waveguide. Results show that there are several distinctive characteristics of the fields scattered by the different mechanisms which depend both on the scattering properties of the bottom and the unperturbed properties of the overall waveguide.
5:00

3pUWa2. In-sediment coherence measurements in a very shallowwater area. Joseph L. Lopes, Iris C. Paustian, and John S. Stroud (Coastal Systems Station, Naval Surface Warfare Ctr., Code R21, 6703 W. Hwy. 98, Panama City, FL 32407-7001)

A measurement was conducted in a very shallow-water (VSW) region to investigate acoustic propagation into a sandy bottom. The measurement utilized several acoustic sources and two identical hydrophone arrays. The sources were mounted onto a stationary sonar tower complete with horizontal pan and vertical tilt motors. Each hydrophone array consisted of four transducers that were buried via water-jetting methods approximately $10 \mathrm{~m}$ from the sonar tower. One array was situated such that the projected acoustic beam was parallel to the rippled structure associated with the sandy bottom, while the other was located where the transmitted beam was perpendicular to the rippled structure. Results of the in-sediment coherence data will be presented as functions of hydrophone burial depth and range in the frequency range between 30 and $150 \mathrm{kHz}$ for the two buried arrays. 
The main physical reasons preventing the generalization of bubbly liquid acoustics in this case are that bubble expansion in unlimited space differs from bubble expansion in porous media, and the most delicate moment of this generalization is how to define the mixture pressure, what the mixture pressure is, and how it corresponds to pressure in the bubbles and relates to bubble dynamics. The goal of this communication is to show how to generalize the bubbly liquid acoustics in the water-bubbles-sand case with the help of the cell-model approach. The cell model is used for the Rayleigh equation derivation for a single bubble dynamics in a porous medium, and the governing equations for nonlinear acoustic waves are derived. The obtained canonical equations can explain some experimental data of three-phase media acoustics.

\section{6:00}

3pUWa5. A mixed wave finite-element approach for solving Biot's poroelasticity equations in acoustics. Franck C. Sgard (LASH, DGCB URA CNRS 1652, ENTPE-Rue Maurice Audin, 69518 Vaulx-en-Velin Cedex, France, Franck.Sgard@entpe.fr), Noureddine Atalla, and Raymond Panneton (Univ. de Sherbrooke, QC J1K 2R1, Canada)

The use of finite-element models for solving Biot's poroelasticity equations often leads to large frequency-dependent systems. Recently, the authors have investigated the use of a selective modal analysis to decrease the size of large finite-element models involving poroelastic structures. The method used a dual real modal basis associated with the skeleton in vасио and the fluid phase occupying the volume of the porous material to approximate the $\{U, p\}$ variables. However, this method converges poorly due to the importance of the damping brought in by the porous material. In this paper, an alternate reduction technique, based on a plane waves expansion method combined with a finite-element approach, is investigated in the case of a single porous layer. The theory behind the technique, together with numerical examples illustrating the performance of the approach, will be presented. [Work supported by Centre Jacques Cartier.]

WEDNESDAY AFTERNOON, 17 MARCH 1999

ROOM H2053, 4:20 TO 6:20 P.M.

Session 3pUWb

\title{
Underwater Acoustics: Scattering from Underwater Structures
}

\author{
George V. Frisk, Chair \\ Woods Hole Oceanographic Institute, Woods Hole, Massachusetts 02543, USA
}

\section{Contributed Papers}

$4: 20$

3pUWb1. Influence of sound-structure interaction on backscattering amplitude in underwater acoustics. H. Peine (FWG, FB 331, Klausdorfer Weg 2-24, 24148 Kiel, Germany)

Sea-based backscattering experiments were carried out in the frequency range from Helmholtz numbers $(k a) 10$ to 30 with a model target. In order to improve target identification, focus here is on separating features related to sound structure interaction from those determined by the target's shape. At the frequencies under concern generally many overlapping vibration modes of the target are excited. Whereas with specially designed objects, like thin-walled hollow cylindrical or spherical shells, isolated resonance modes can be directly observed in the echo and assigned to surface waves, the model target used here resembles the complex structure of real obstacles. The structural responses measured by accelerometers attached inside the model are recorded simultaneously together with the backscattering amplitude allowing immediate comparison. Narrow-band swept sine and $\mathrm{CW}$ pulses were used. At each frequency the target was turned around a fixed axis with angle resolution better than 2 degrees. A novel spectral transfer function is tested modeling the frequency-dependent boundary impedance and therefore the envelope deformation of the scattered pulse due to interaction of the impinging wave with the target's interior. This model function has rather few parameters to be adjusted.

4:40

3pUWb2. A perturbation theory for scattering from targets near rough surface sediments: Numerical results. Garner C. Bishop and Judy Smith (Naval Undersea Warfare Ctr., Div. Newport, Newport, RI 02840)

A null field $T$-matrix perturbation theory developed for plane-wave scattering from a fluid loaded elastic spherical shell near a rough fluid elastic interface [J. Acoust. Soc. Am. 101, 767-788 (1997)] is generalized to include scattering from spherical, spheroidal, and endcapped cylindrical targets near rough surface rigid, soft, fluid, and elastic sediments. Numerical results are obtained that demonstrate some of the effects of surface roughness on resonant and nonresonant target-boundary scattering. 
3pUWb3. Localized modes of oscillating structures which lie on the bottom. Andrei Abramian (Inst. for Problems in Mech. Eng. RAS, Bolshoy pr. V. O. 61, 199178 St. Petersburg, Russia, abramian@hidro.ipme.ru)

The phenomenon of wave localization in hydroelastic systems leads to the strength concentration of radiation fields. The linear method considers the process of localization to be the formation of nonpropagation waves (trapped modes phenomenon). The presence of such waves in the total wave packet points to the existance of mixed natural spectrum of differential operators describing the behavior of hydroelastic systems. The problem of liquid and oscillating structure interaction caused by the trapped modes phenomenon has been solved (membranes, dies, plates and different liquid models). The areas of wave localization have been determined. These areas depend on both the parameters of oscillating structures and the liquid waveguide parameters (the linear dimensions of the channel filled with liquid; the height from the bottom to the liquid surface; the liquid density gradient according to the height, etc.). Necessary and sufficient conditions for the low-frequency trapped modes and the highfrequency trapped modes have been found. The wave localization in nonlinear elastic structures interacting with liquid has been studied. Cases of weak and strong nonlinearity have been investigated. It has been shown that the slightly decreasing tail is added to nonlinear localized modes depending on the frequency of linear localized modes. The tail is caused by the interaction of the mode's continuous spectrum and the localized modes.

\section{$5: 20$}

3pUWb4. Trapping modes of oscillations in an infinitely long waveguide with submerged object in the form of a massive die. Dmitri Indeitsev (Inst. for Problems in Mech. Eng. RAS, Bolshoy pr. V.O. 61, St. Petersburg 199178, Russia, abramian@hidro.ipme.ru)

It is shown for an infinitely long waveguide-first in the form of a two-dimensional and then a three-dimensional channel-filled with an ideal compressible liquid with a free surface, that there can exist standing waves as well as traveling waves localized in the region of a dynamic submerged object-a massive die. The appearance of such waves is due to the presence of a mixed spectrum of characteristic frequencies of oscillation. In addition to the continuous spectrum, a point (discrete) spectrum distributed along the axis of the continuous spectrum is also present. A relation is found between the geometric parameters of the channel and the massive die for which this spectrum exists.
3pUWb5. Repulsion phenomena in the phase-velocity dispersion curves of circumferential waves on elastic cylindrical shells. Herbert Überall (Dept. of Phys., Catholic Univ., 6th and Michigan Ave. N.E., Washington, DC 20064), Gérard Maze, Fernand Léon, and Jean Ripoche (Univ. of Le Havre, 76610 Le Havre, France)

The complex eigenfrequencies of an evacuated, fluid-immersed infinite cylindrical shell, when plotted in the complex frequency plane, have been grouped into families corresponding to different types of circumferential waves. The real parts of these eigenfrequencies have been used to obtain dispersion curves versus frequency of the phase velocities of these circumferential waves, which are analogous to those of the Lamb waves on an elastic plate in vacuo labeled $A_{0}, A_{1}, \ldots$ and $S_{0}, S_{1}, \ldots$, but which contain an additional branch reminiscent of the fluid-borne Scholte-Stoneley wave. This branch forms together with the $A_{0}$-analog an interacting pair of dispersion curves which will be called $A_{0+}$ (upper branch) and $A_{0-}$ (lower branch). These exhibit a repulsion phenomenon near the ambient fluid sound speech analogous to that in perturbed, quasi-degenerate atomic levels. This is explained here by perturbation theory, showing that during repulsion the wave character gets exchanged so that at frequencies above the repulsion point $A_{0+}$ is a flexural and $A_{0-}$ a fluid-borne wave, and vice versa below the repulsion point.

\section{6:00}

3pUWb6. Localization in acoustic propagation in water with air cylinders. Zhen Ye and Emile Hoskinson (Dept. of Phys., Natl. Central Univ., Chungli, Taiwan, ROC)

Wave localization is the peculiar property of a scattering medium that completely blocks propagation, thus inducing a surprising phase transition, for example in optical or acoustic transparency or electrical conductivity. When localized, waves remain confined in space until dissipated. This property has been explained for randomly disordered scatterers. It is generally believed that disorder is an essential ingredient of localization, and in two dimensions (2D) waves are always localized with any amount of randomness. Here it is shown that waves are not always localized in 2D, but under proper conditions even periodic structures can achieve localization in the case of acoustic propagation through water containing an array of air cylinders. An essential component of localization in this case arises from the natural resonance and an amazing collective behavior of the cylinders. Exact numerical calculations reveal the unexpected result that localization is relatively independent of the precise location or organization of the scatterers. The results provide insight on 2D wave localization and offer an opportunity for further investigation of important questions about localization phenomena. [Work supported by NSC and NCU of ROC.] 